\title{
WestVirginiaUniversity
}

THE RESEARCH REPOSITORY @ WVU

Graduate Theses, Dissertations, and Problem Reports

2001

\section{Optical gradation for crushed limestone aggregates}

\author{
Ken Cheng \\ West Virginia University
}

Follow this and additional works at: https://researchrepository.wvu.edu/etd

\section{Recommended Citation}

Cheng, Ken, "Optical gradation for crushed limestone aggregates" (2001). Graduate Theses, Dissertations, and Problem Reports. 2318.

https://researchrepository.wvu.edu/etd/2318

This Dissertation is protected by copyright and/or related rights. It has been brought to you by the The Research Repository @ WVU with permission from the rights-holder(s). You are free to use this Dissertation in any way that is permitted by the copyright and related rights legislation that applies to your use. For other uses you must obtain permission from the rights-holder(s) directly, unless additional rights are indicated by a Creative Commons license in the record and/ or on the work itself. This Dissertation has been accepted for inclusion in WVU Graduate Theses, Dissertations, and Problem Reports collection by an authorized administrator of The Research Repository @ WVU.

For more information, please contact researchrepository@mail.wvu.edu. 


\title{
Optical Gradation for Crushed Limestone Aggregates
}

\author{
Ken Cheng \\ Dissertation submitted to the \\ College of Engineering and Mineral Resources \\ of \\ West Virginia University \\ in partial fulfillment of the requirements \\ for the degree of \\ Doctor of Philosophy \\ in \\ Mechanical Engineering \\ Larry E. Banta, Ph.D., Chair \\ John P. Zaniewski, , Ph.D. \\ Nigel Clark, Ph.D. \\ Mark Jerabek, Ph.D. \\ James Smith, Ph.D. \\ Department of Mechanical and Aerospace Engineering \\ Morgantown, West Virginia \\ 2000
}

Keywords: Aggregates gradation, Machine vision, Image processing, Sieving, Limestone 


\section{ABSTRACT \\ Optical Gradation for Crushed \\ Limestone Aggregates}

Ken Cheng

The strength and durability of asphalt pavement is directly affected by the characteristics of its main ingredient, mineral aggregate. Besides material strength, research has shown that mixture properties such as particle shape and mixture gradation have a significant affect on the quality of the asphalt concrete. A standard called "Superpave" has been developed which sets forth specifications for material selection and methods for measurement of aggregate properties. These standards require monitoring of aggregate properties, particularly gradation. In this dissertation, the feasibility of developing an optically based method for determining aggregate gradation was explored. The physical system primarily consists of a standard monochrome CCD video camera and a computer with a frame grabber board. Software was developed to separate touching or overlapping particles in the image, and to detect the size and shape of each particle. Correlation to estimate each particle's mass and to predict the sieving behavior for crushed limestone aggregates was developed and tested. Laboratory testing demonstrated the ability to measure gradation over a range of particle sizes from $4.75 \mathrm{~mm}$ to $25 \mathrm{~mm}$ with an accuracy of \pm 3 in terms of percent-passing residual when compared with mechanical sieving. 


\section{TABLE OF CONTENTS}

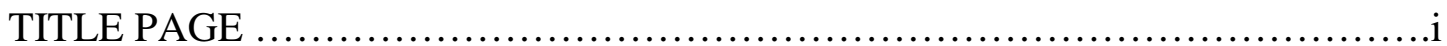

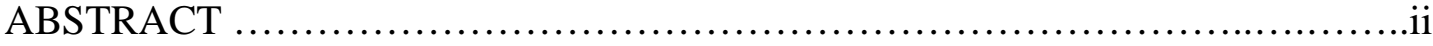

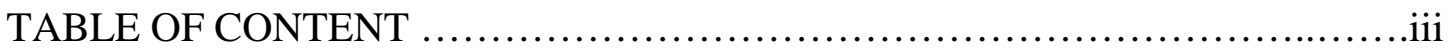

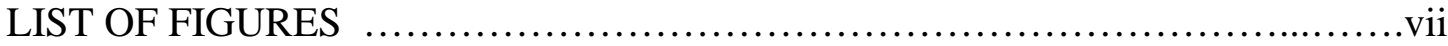

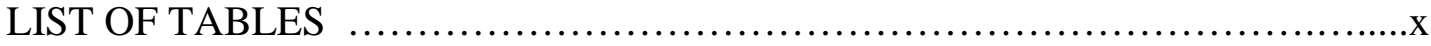

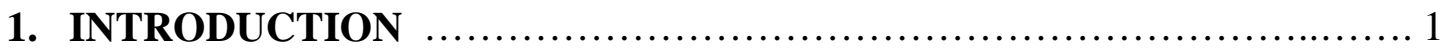

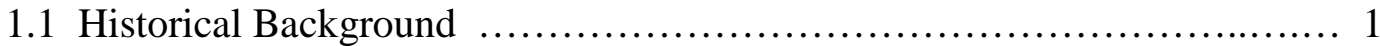

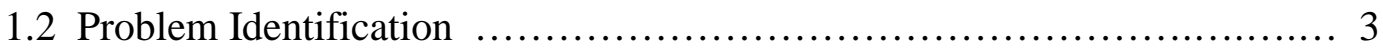

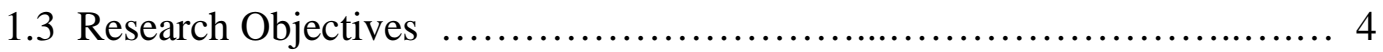

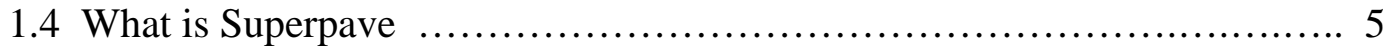

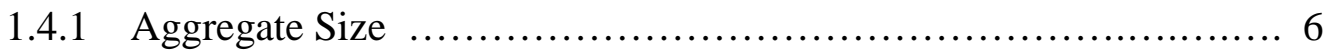

1.4.2 Aggregate Shape $\ldots \ldots \ldots \ldots \ldots \ldots \ldots \ldots \ldots \ldots \ldots \ldots \ldots \ldots \ldots \ldots \ldots$

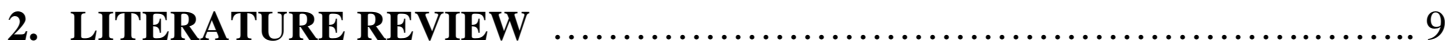

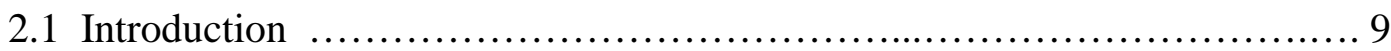

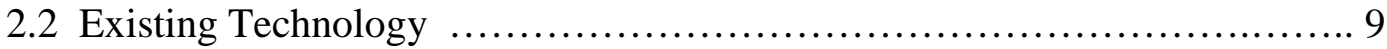

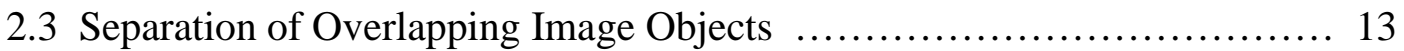

2.4 Particle Passage Probability in Sieving ............................... 14

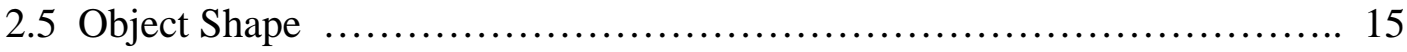

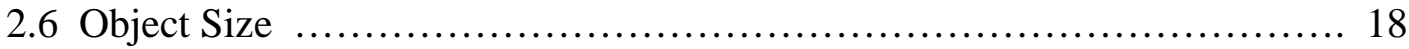

3. LABORATORY SET-UP AND MEASUREMENT CALIBRATION .......20

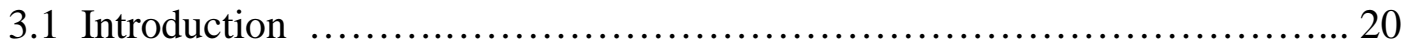

3.2 Hardware Set-up and Operating Condition $\ldots \ldots \ldots \ldots \ldots \ldots \ldots \ldots \ldots \ldots \ldots \ldots \ldots \ldots \ldots \ldots$

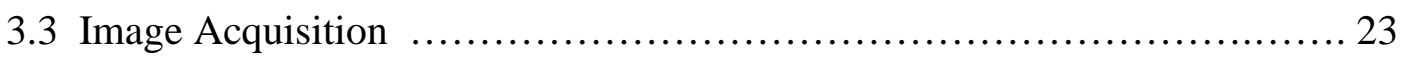

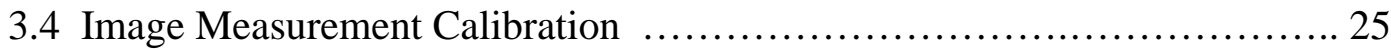




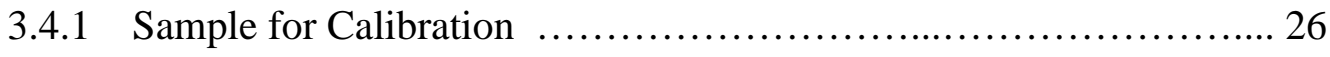

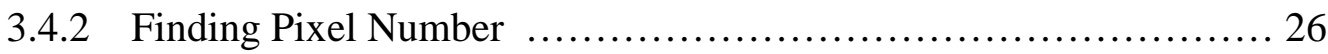

3.4.3 Area Correction ............................................... 28

4. IMAGE PROCESSING AND ANALYSIS .................................. 32

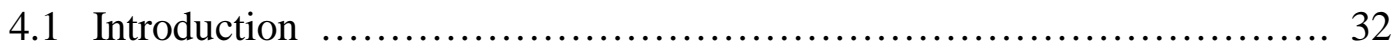

4.2 General Description ................................................ 33

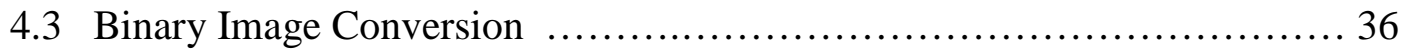

4.4 Object Detection and Seeding .......................................... 37

4.5 Edge Detection, Region Growing and Particle Projected Area .............. 39

4.6 Centroid Location ........................................................ 41

4.7 Major and Minor Diameter Computation …............................. 46

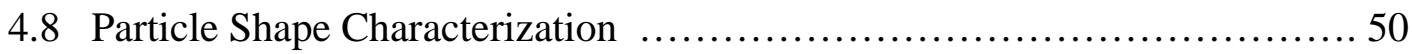

5. SEPARATION OF TOUCHING AND OVERLAPPING PARTICLES....... 57

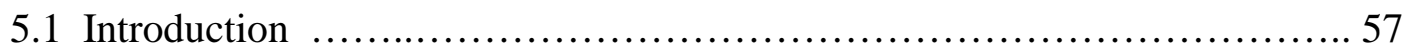

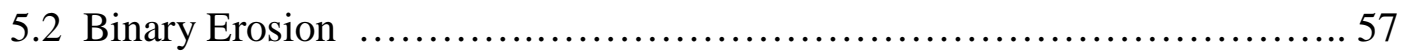

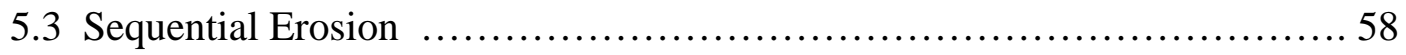

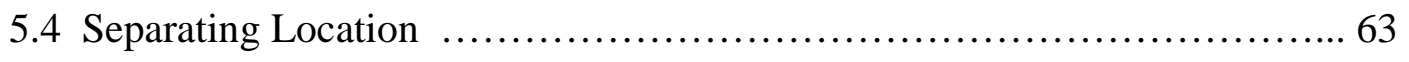

5.5 Detection of Saddle Point by Filtering …............................... 67

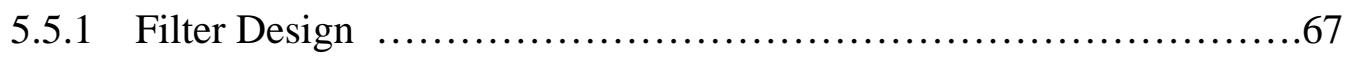

5.5.2 Saddle Point Conditions ............................................. 69

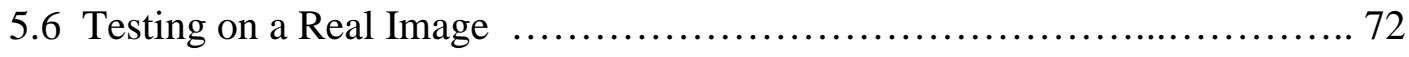

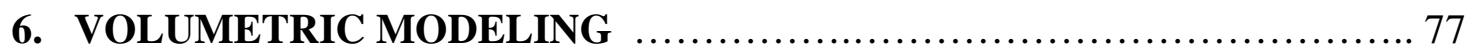

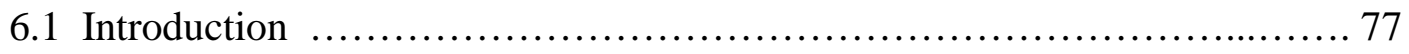

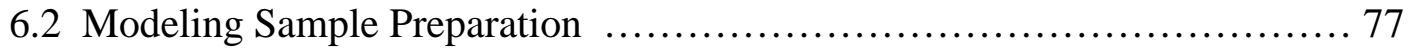

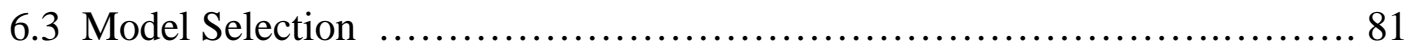

6.4 Particle Geometric Assumptions and Definition ............................. 83

6.5 MLR Model Building .................................................. 85

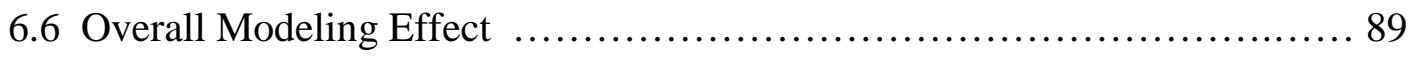




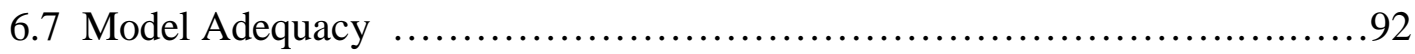

6.7.1 The Coefficient of Multiple Determination .......................... 92

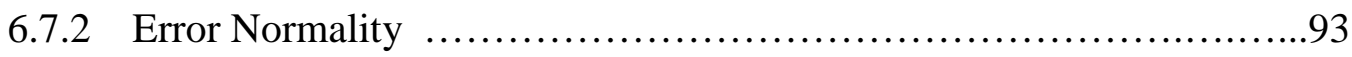

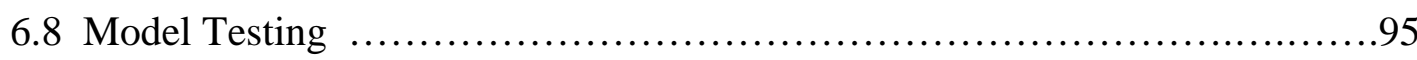

6.8.1 Preparation of Testing Sample Population …......................96

6.8.2 Testing Results ..................................................97

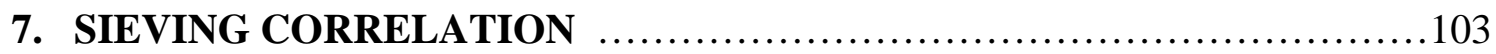

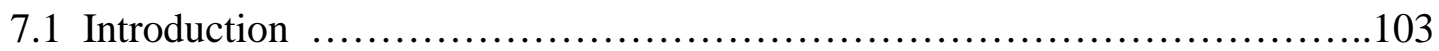

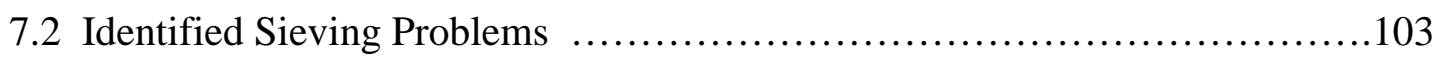

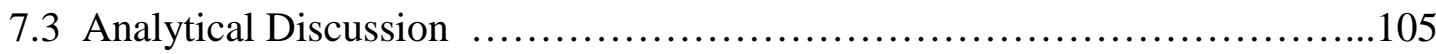

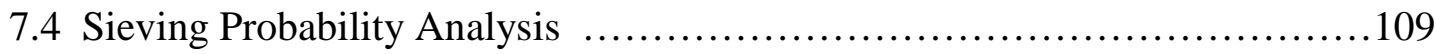

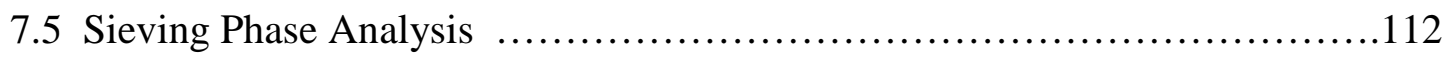

7.5.1 Size Modification for Triangular Shapes ...........................112

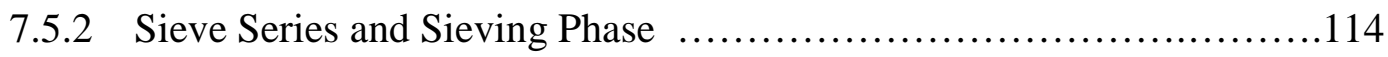

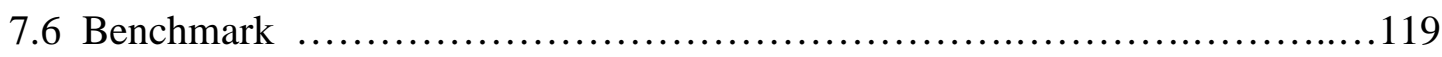

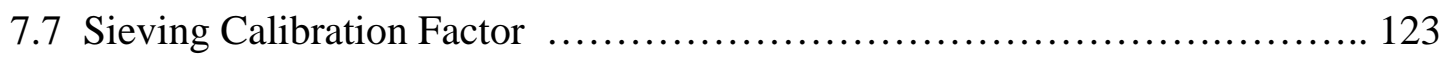

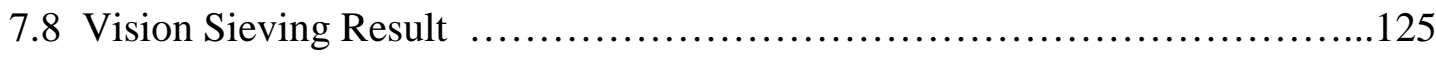

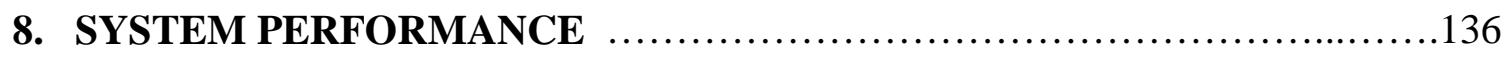

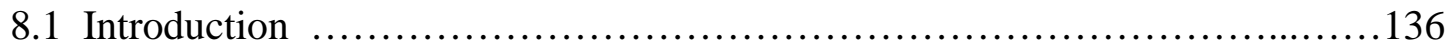

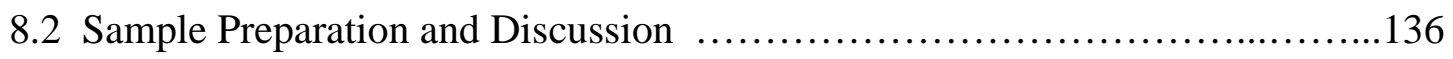

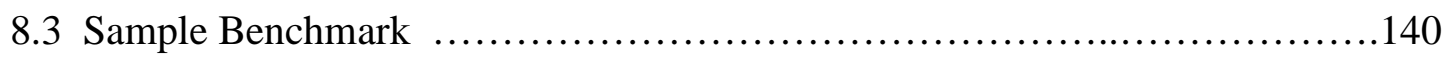

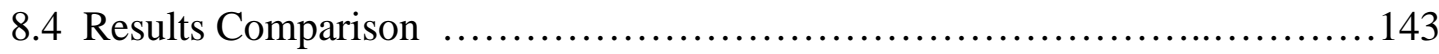

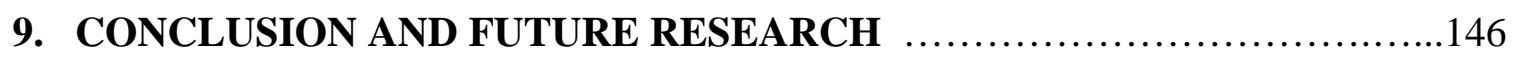

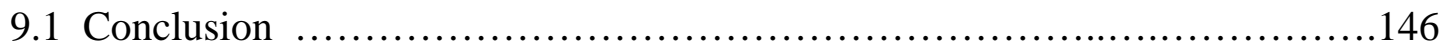

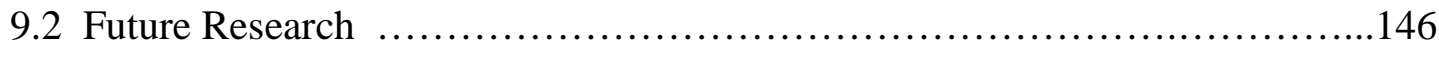




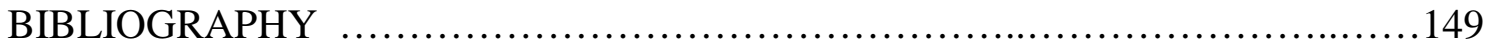

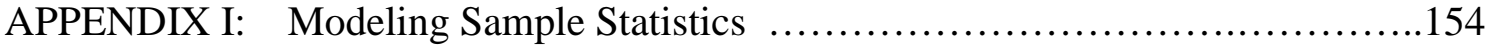

APPENDIX II: Histogram of Model Testing Samples $\quad$..............................

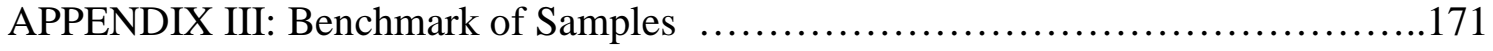

APPENDIX IV: Images of Samples for Testing System Performance .................175

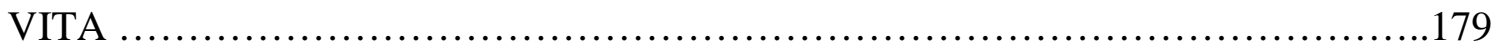




\section{TABLE OF FIGURES}

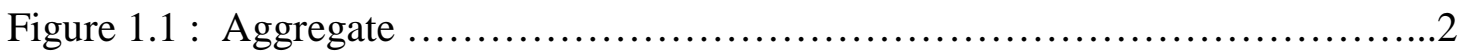

Figure 1.2 : Proportional Calipers ...........................................

Figure 1.3 : Graphical Basis for 0.45 Power Chart ................................

Figure 1.4 : Graphical Basis for Actual Size Chart ..............................

Figure 2.1 : Aggregate Size by Superpave ...................................19

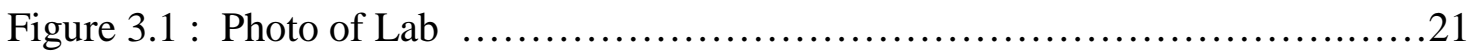

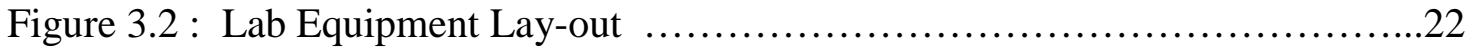

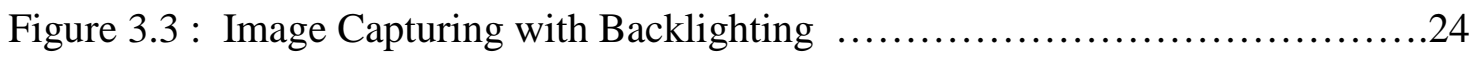

Figure 3.4 : Image Acquisition and Processing System ...........................25

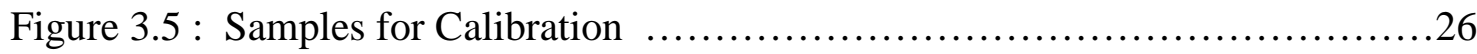

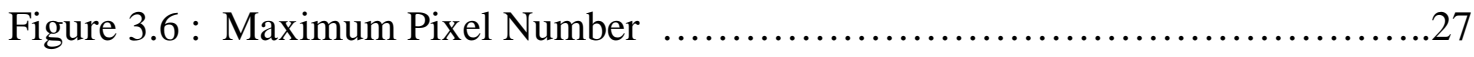

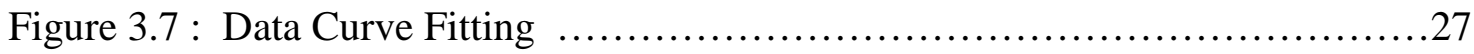

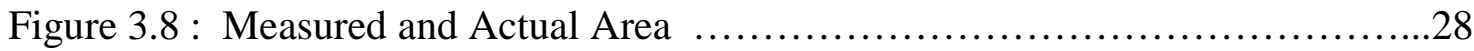

Figure 3.9 : Curve Fitting for Measured Area .................................29

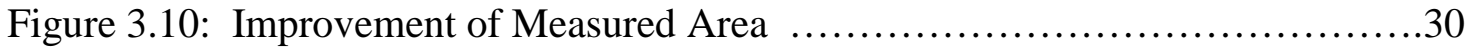

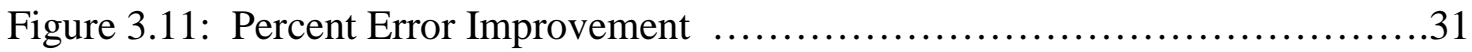

Figure 4.1 : Simulated Particles with Centroid, Edge and Interior Points Labeled ......33

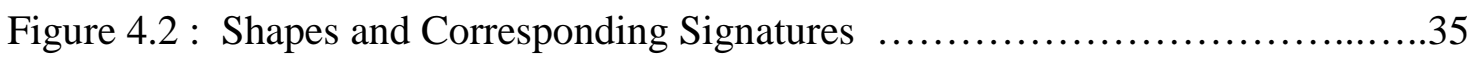

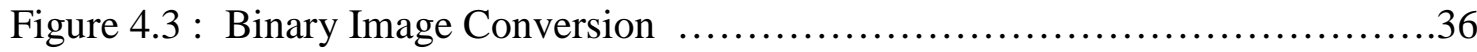

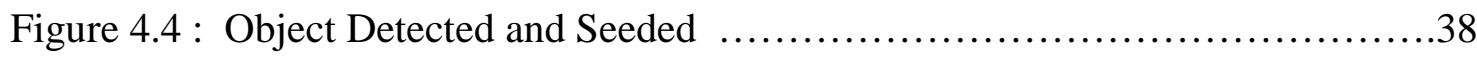

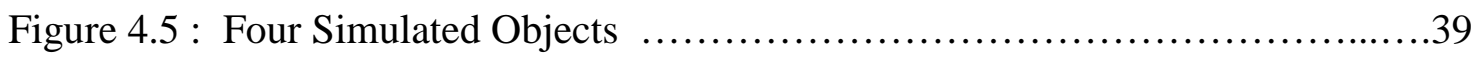

Figure 4.6 : Sequence of Multi-object Detection and Seeding .....................39

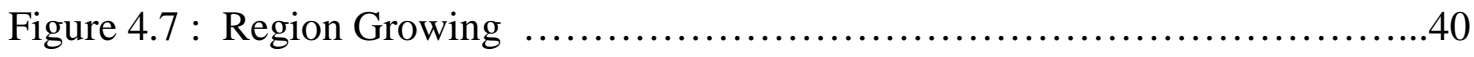

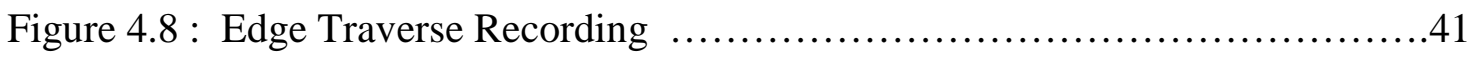

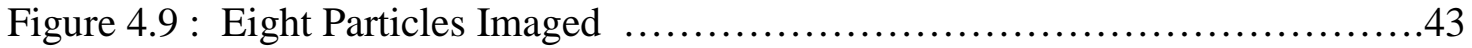

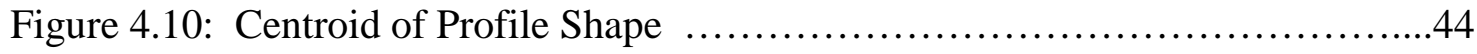

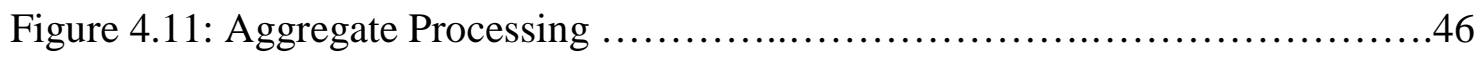

Figure 4.12: Major and Minor Diameter ....................................47

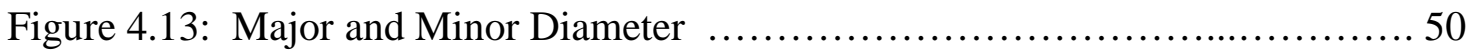




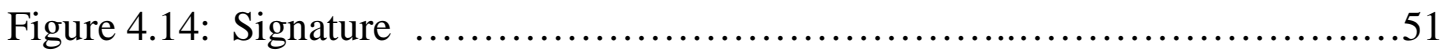

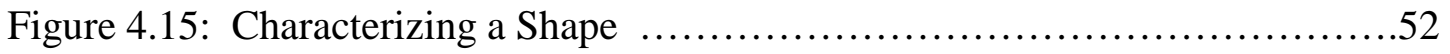

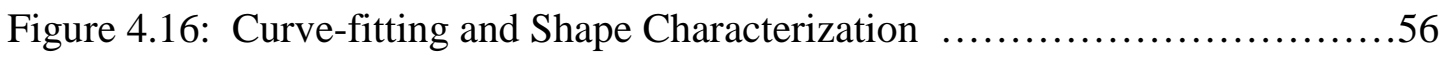

Figure 5.1 : Typical Erosion as Shrinking ....................................58

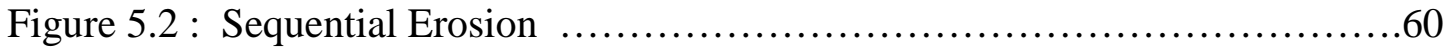

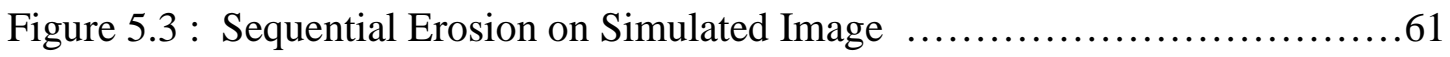

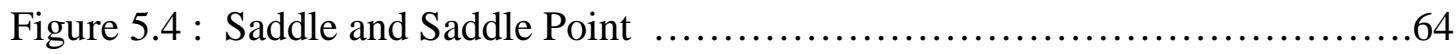

Figure 5.5 : Dissecting Plans for Finding Saddle Point ........................66

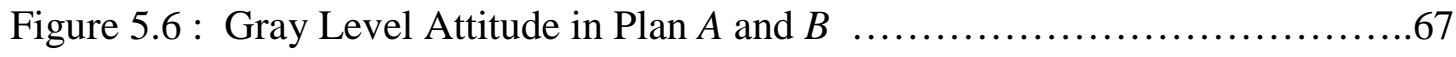

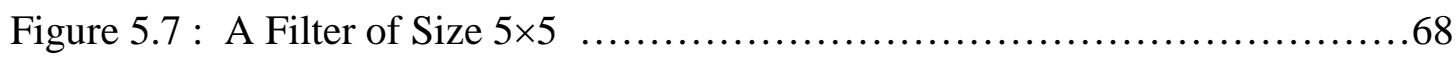

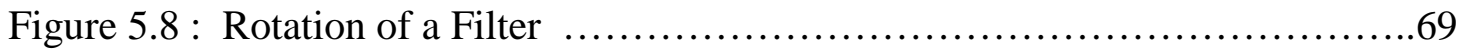

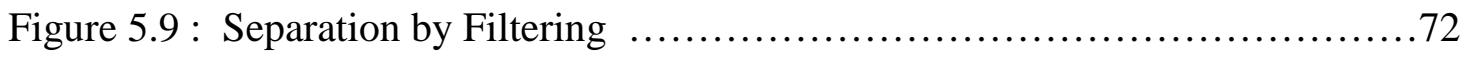

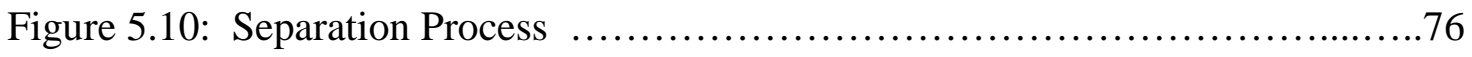

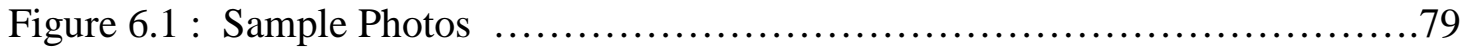

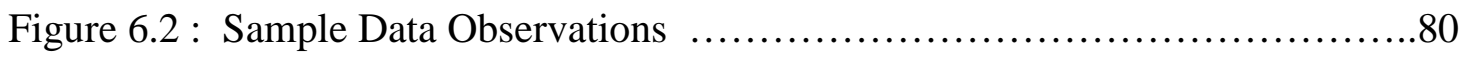

Figure 6.3 : Geometric Perspective of Particle .................................84

Figure 6.4 : Interpretation of Model Variables $\ldots \ldots \ldots \ldots \ldots \ldots \ldots \ldots \ldots \ldots \ldots \ldots . \ldots 7$

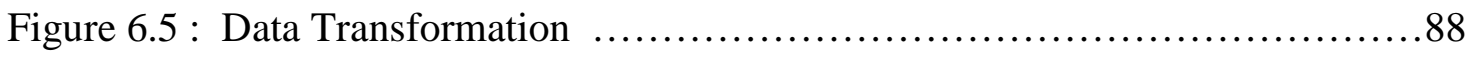

Figure 6.6 : Volume Comparison .........................................91

Figure 6.7 : Modeling Effect Observation .....................................92

Figure 6.8 : Model Error vs. Particle Sequence $\ldots \ldots \ldots \ldots \ldots \ldots \ldots \ldots \ldots \ldots \ldots \ldots . \ldots 9$

Figure 6.9 : Histogram of Standardized Residuals . ...........................95

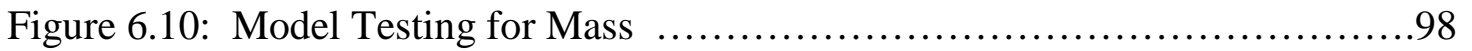

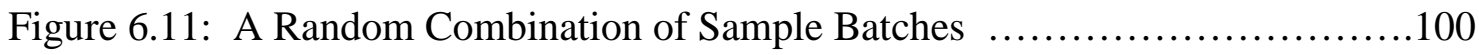

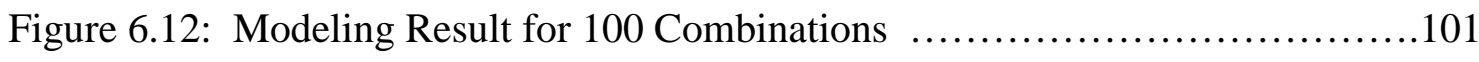

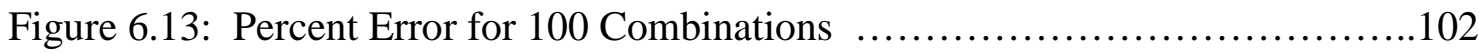

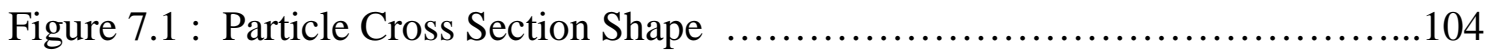

Figure 7.2 : Particle Passing Mechanism ...................................... 105

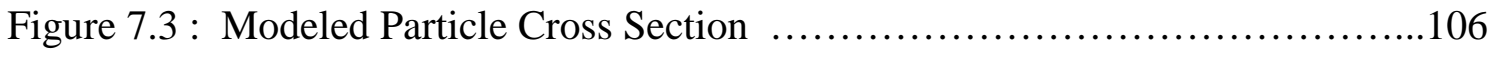

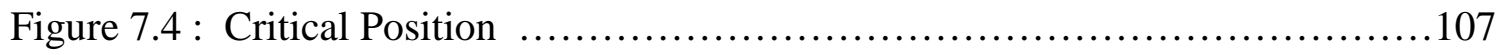

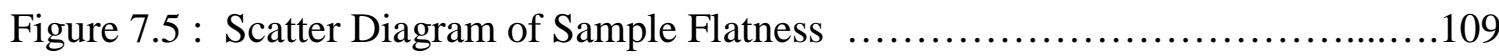




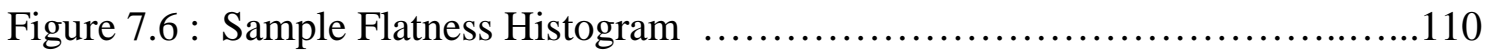

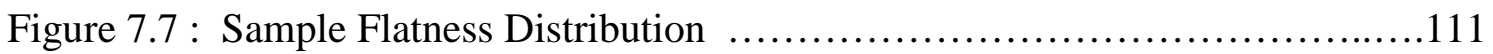

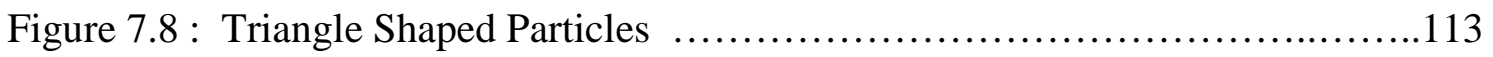

Figure 7.9 : Physical Sieve Cascade .............................................115

Figure 7.10: Overview of Sieving Phase Analysis ................................116

Figure 7.11: Percent Retained and Percent Passing for Sample Batch \#4 ..............121

Figure 7.12: Mass Change in Sieving Process …................................ 122

Figure 7.13: Benchmark for Sample Batch \#4 ….................................123

Figure 7.14: Benchmark for Combined Sample Batches ….......................125

Figure 7.15: A Random Combination of Sample Batches ............................127

Figure 7.16: Sieving Correlation Testing \#1 f....................................130

Figure 7.17: Sieving Correlation Testing \#2 …..................................131

Figure 7.18: Sieving Correlation Testing \#3 …..............................132

Figure 7.19: Sieving Correlation Testing \#4 …................................133

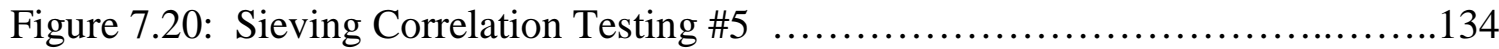

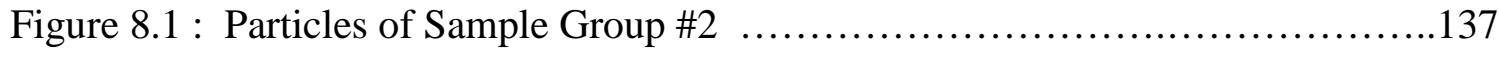

Figure 8.2 : Histogram of Measured Diameter ..................................138

Figure 8.3 : Histogram of Estimated Flatness …..................................139

Figure 8.4 : Histogram of Estimated Volume …................................139

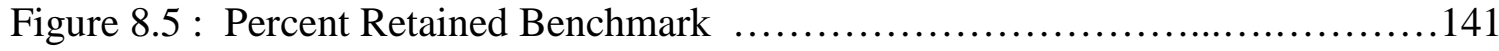

Figure 8.6 : Percent Passing Benchmark .........................................142

Figure 8.7 : Percent Retained Correlation ….....................................144

Figure 8.8 : Percent Passing Correlation ......................................... 145 


\section{LIST OF TABLES}

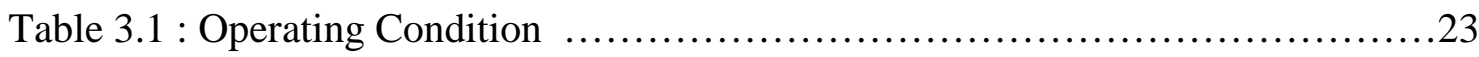

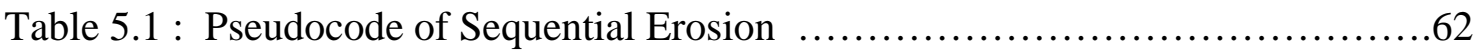

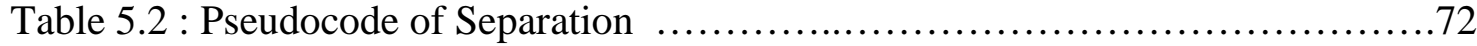

Table 6.1 : Sample Measurement Statistics ................................. 96

Table 7.1 : Pseudocode of Sieving Procedure .................................119

Table 7.2 : Benchmark Values for Combined Sample Batches $\ldots . \ldots \ldots \ldots \ldots \ldots \ldots \ldots . . . \ldots 125$

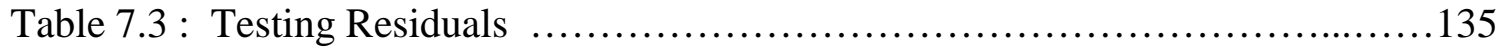

Table 8.1 : Statistics of the Benchmark (Sieving of 10 times) $\ldots . \ldots \ldots \ldots \ldots \ldots \ldots . \ldots 142$

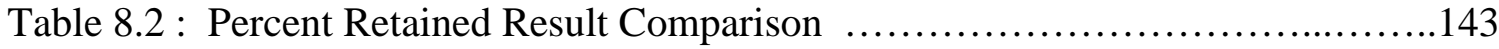

Table 8.3 : Percent Passing Result Comparison ......................................144 


\section{INTRODUCTION}

\subsection{Historical Background}

Hot-mix asphalt concrete is widely used to build modern highways. The strength and durability of asphalt concrete pavements are profoundly affected by the characteristics of the aggregates. Beyond the obvious dependence on aggregate's properties such as the strength and durability, characteristics such as particle shape, and gradation (i.e., size distribution) are extremely important. Research performed as part of the Strategic Highway Research Program provided a standard for asphalt concrete mix design called "Superpave" [1], which specifies limits for aggregate gradation, particle angularity, and percentage of thin and elongated particles.

The particle size distribution in the mixed asphalt plays a vitally important role in the quality control for the highway building. For instance, pavements constructed with too high a percentage of fine particles such as natural sand will display unallowable levels of permanent deformation when loaded by traffic. On the other hand, too many large particles in the mixed asphalt can produce a large amount of voids. As a result, the strength and durability of the pavement will be compromised. The quality of pavement demands the appropriate mixture of various sizes of particles, and the size distribution of the mixture is presented by the gradation curve.

Particle shape is also important because rough or angular aggregates provide more strength than rounded, smooth-textured aggregates as shown in Fig. 1.1. Even though a jagged piece and a rounded piece of aggregate may possess the same material strength, angular aggregate particles tend to lock together resulting in a stronger mass of material. On the other hand, rounded aggregate particles tend to slide by each other. Flat and 
elongated aggregates tend to break during handling, construction and under traffic load, changing the design gradation and compromising strength.

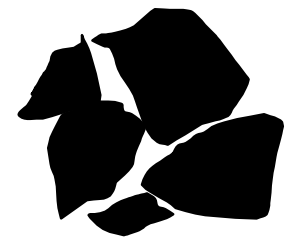

(a) Angular aggregates tend to lock

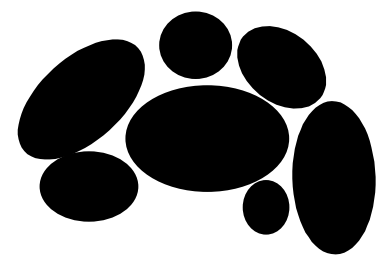

(b) Rounded aggregates tend to slide

Figure 1.1 Aggregate

Traditional methods for evaluating size, shape and texture of aggregates are timeconsuming and labor intensive. Until very recently, some of aggregate evaluation for pavements was done manually. Individual coarse aggregate particles are visually examined to determine the number of fractured faces per particle. For determination of a particle's flatness and elongation, a proportional device is used, as illustrated in Fig. 1.2. The aggregate particle is first placed with its largest dimension between the swinging arm and fixed post at position $A$. The swinging arm is locked in position, the same aggregate is placed between the swinging arm and post at position $B$. If the aggregate passes this gap, then it is counted as a flat or elongated particle. Procedures such as these are timeconsuming and are limited to a small sample sizes. There is no possibility of using these methods to provide real-time feedback for process control. 


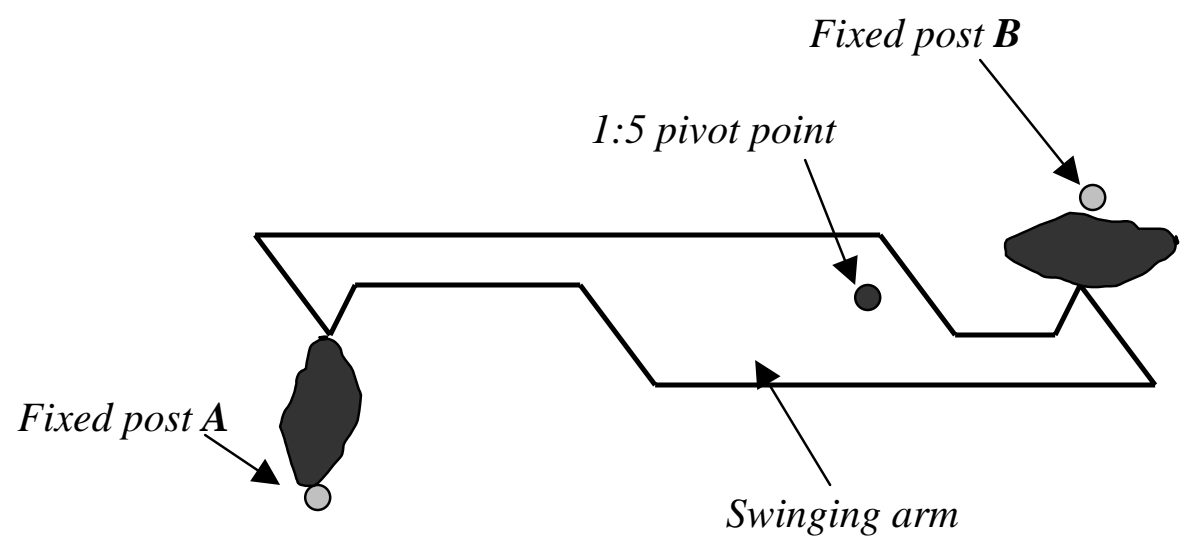

Figure 1.2 Proportional Calipers

Some of drawbacks existing in the traditional method can be overcome by taking advantage of modern machine vision techniques. It is believed that through processing and analyzing the aggregate images captured by machine vision system (a video camera, a frame grabber, and a PC), the size distribution, shape and angularity of aggregates can be determined. Instead of the mechanical sieving, "vision sieving" may be used to achieve results close to those obtained mechanically. Vision sieving offers two potential improvements over the traditional methods: reduced level of manual involvement and potential for automated gradation control.

\subsection{Problem Identification}

This work investigates the feasibility of using non-contact optical methods to provide information generally obtained by mechanical methods. Three particularly difficult problems arise within using machine vision for aggregate evaluation:

1) Particles are usually touching, overlapping, or even entirely occluded. Rapid discrimination of one particle from another is both necessary and difficult. 
2) Standards for classifying particles by size are generally based on mechanical sieving and the process results depend on a combination of both size and 3dimensional shape of particles. It is desirable to avoid the complexity and expense of explicitly measuring the $3^{\text {rd }}$ dimension of each particle.

3) Sieving standards are also set up to report particle gradation on a "percent passing" basis, where the fraction is based on mass. So in addition to extrapolation of the interaction between a particle's 2-D features and the sieving process, it is necessary to develop a means to extrapolate the relationship between a particle's 2-D features and its volume. These extrapolations will be dependent on general size and shape properties that vary from particle to particle. For example spherical particles will have different sieving and volume transformation than cylindrical, cubic, or triangular particles.

The fundamental question is then, "Can we extract a set of features from the 2-D image which will provide adequate information to accurately predict volume characteristics, elongation, angularity, and the sieving behavior from the particles' 2-D video image?"

\subsection{Research Objectives}

The work can be broken into three major tasks as follows:

1) To effectively describe the sieving characteristics of 3-D aggregates based on 2-D geometric size and shape of the particles. 
2) To develop a functional relationship between a particle's plan features and its corresponding volume. In other words, inferring volume information of the 3 D particle under consideration by means of measurements obtained from 2-D image. This will be the main theme of this research.

3) To develop a simple and efficient method that can separate the touching and overlapping particles in the scene.

\subsection{What is Superpave?}

From 1987 through 1992, the Strategic Highway Research Program (SHRP) conducted a research effort to develop new ways to specify, test, and design asphalt materials. After 1992, the Federal Highway Administration (FHWA) assumed a leadership role in the implementation of SHRP research. An essential part of FHWA's implementation strategy was educating agency and industry personnel in the proper use and application of the final SHRP asphalt products, collectively referred to as Superpave [1].

Definitions for properties of aggregate such as size, shape and texture may vary from standard to standard, depending on the agencies involved. However, because this research is a project aimed at improving methods of aggregate gradation and shape identification, size, shape and other related definitions given in the Superpave guide book have become the guidelines in terms of comprehending the aggregate's characteristics. 


\subsubsection{Aggregate Size}

Many technical reports in the field of mineral aggregate property studies explicitly or implicitly regard the area of the particle in a 2-D plane as particle size $[3,4$,

5, 6]. In Superpave [1], the aggregate size is considered as being the dimension of a square sieve opening through which the particle falls by its own gravity. Let a sieve size be a square of $D_{i} \times D_{i}$, where $D_{i}$ takes a discrete value of a sequence with $D_{N}>D_{N^{-1}}>D_{N \text { - }}$ ${ }_{2} \ldots>D_{l}$. The aggregate size $d$ is then a value that satisfies

$$
D_{i-1}<d \leq D_{i}
$$

Superpave prefers to use the 0.45 power gradation chart to define an allowable gradation limits. This chart uses a unique graphing technique to judge the cumulative particle size distribution of a blend of aggregates. The ordinate of the chart is percent passing, the abscissa is an arithmetic scale of sieve size in millimeters, raised to 0.45 power. Fig. 1.3 illustrates how the abscissa is scaled. In this example, the $4.75 \mathrm{~mm}$ sieve is plotted as 2.02 units to the right of the origin. 


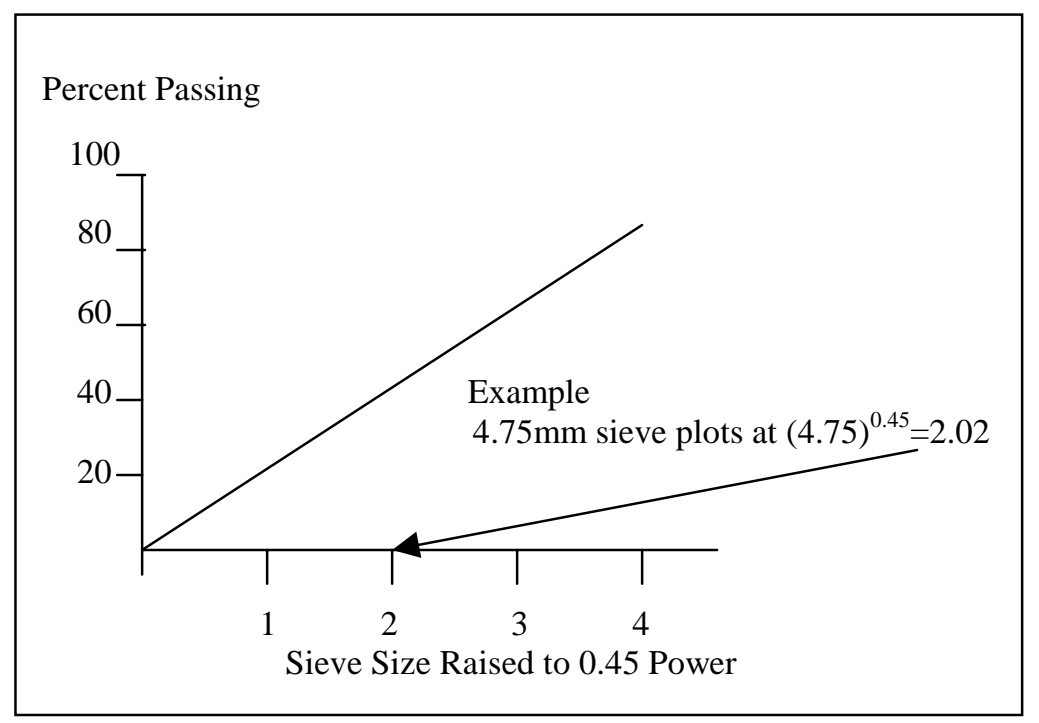

Figure 1.3 Graphical Basis for 0.45 Power Chart

For the sake of convenience, in this work an alternative way of construction of percent passing curve is used: the ordinate of the chart is still percent passing, but the abscissa indicates the actual sieve size in millimeters, as shown in Fig. 1.4.

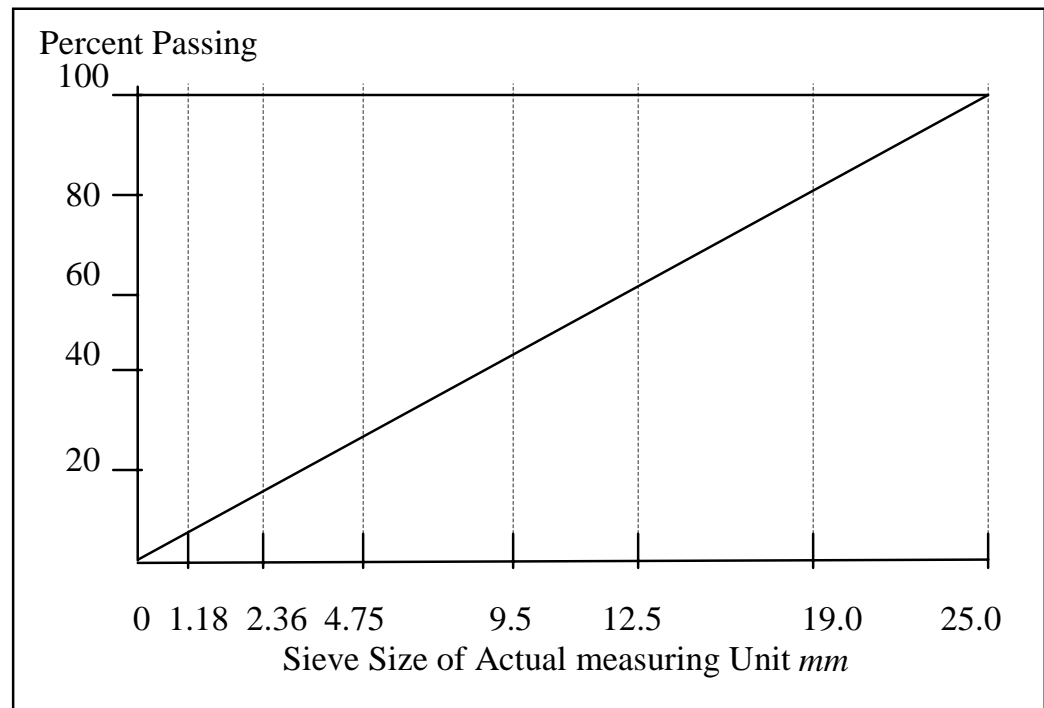

Figure 1.4 Graphical Basis for Actual Sieve Size Chart 


\subsubsection{Aggregate Shape}

The Superpave manual [1] describes the particle shape as:

- Flat and elongated: The ratio of a maximum to minimum dimension is greater than 5 .

The aspect ratio of the particle can be used for detection of elongated shape. Aspect ratio is defined as the ratio of the maximum diameter to the orthogonal minimum diameter of the shape silhouette.

In Superpave, shape identification is performed by obtaining the percentage by mass of coarse aggregates that are elongated. Elongated particles are undesirable because they have a tendency to break during construction and under traffic. 


\section{LITERATURE REVIEW}

\subsection{Introduction}

This research is associated with many aspects in the fields of image processing, image analysis and statistics. Related work in image processing mainly involves image segmentation, more specifically, separation of touching and overlapping shapes, and object size and shape characterization. To optically "sieve" the particles, it needs to predict the particle mass based on 2-D image measurements.

There are many publications on image edge detection, image size and shape analysis. Many techniques in shape characterization such as Fourier analysis and template matching have been reported in literature. Some novel methods such as polygonal harmonics are also attracting attention. By comparison, fewer articles regarding separation of the touching and overlapping imaged shapes exist. There are some reports about inferring the objects' 3-D information (volume) from their 2-D measurements. Some insights into optical sieving may be shared from the reports on existing technology, and several video graders using these technologies have been marketed commercially.

\subsection{Existing Technology}

In searching for the work related to this research project, only three commercial products that perform the functions desired for Superpave quality control were discovered. There are some helpful descriptions of these three commercially available systems given by H. Kim, et al [33]. 
The EMACO corporation of Montreal, Canada markets a device called the VDG $40^{\mathrm{TM}}$, which uses optical methods to perform particle sieving. The VDG 40 employs a line-scan camera and approximates particle boundaries by drawing successive chords across the particles falling off the vibrating feeder. Although there has been some debate about its accuracy by some independent testers [32], this system is claimed to perform the following functions:

- Produce gradation curves for particles whose sizes range from 1 to $50 \mathrm{~mm}$.

- Calculate mean elongation coefficient.

- Estimate the "flattening coefficient".

- Uncertainty less than $1.7 \%$ for samples with enough particles in each class.

Based upon the assumption that the thickness of the particle is the same as its width, the volume is computed using an ellipsoid of revolution [32]. No clear information about how to separate the touching and overlapping is provided even though the falling particles may be overlapped when viewed in any direction. A description of the on-going effort on testing and improving VDG 40 is summarized by R.L. Weingart, et al [31].

Several products are marketed by the WipWare Company in Bonfield, Ontario, Canada $[35,39]$. WipFrag ${ }^{\mathrm{TM}}$ performs optical gradation on bulk materials on the ground or on moving conveyor belts. The WipFrag system is based on area scan video cameras. Some case studies using the Wipfrag image analysis system were presented by Maerz [40]. Another product, WipShape, uses a conveyor and two video cameras to image one particle at a time and compute the percentage of flat, elongated particles [35, 37]. 
N.H. Maerz used stereology and object geometric probability to explore the possible solution of the problem of inferring the true size distribution of a body of particles, given the observed profile distribution on an imaged scene [27]. The process is known in stereology as "unfolding" a distribution. The problem is: can one reconstruct a block size distribution of a pile of blast fragmented rock from a measurement made on the surface of that pile? Maerz found that if one applies the stereological theory developed by previous researchers to this problem, many of the assumptions made for the existing theory are violated. Therefore, Maerz suggested a new method of unfolding the distribution.

This new method is based on analyzing fragmentation using image analysis, and first assuming all particles to be spherical for a quick solution. The distribution from the image can be calculated. Maerz states that the observed distribution should be further divided into a number of classes, in each of which the particles have a similar diameter. A calibration function was added to account for numerous effects to improve algorithm's accuracy. This makes the equation become "semi-empirical". The calibration function is determined by back calculation from a known size distribution.

An experimental system is under development by Rao, et al at the University of Illinois [32]. Rao developed an experimental device that uses three cameras to capture orthogonal images of a single particle at a time. Rao's objective is to improve the detection of flat, elongated particles, and it was claimed that the system performs more accurately than either the VDG 40 or the WipFrag system [32]. The tests have demonstrated volume measurement errors ranging from 5\% to more than $10 \%$, but errors in detection of flat, elongated particles were within approximately $1-2 \%$. Rao's device is 
quite slow, however. A processing time for 1037 particles of 70 minutes was reported. The volume errors are also relatively high $(\approx 10 \%)$ when compared with the published claims of commercially available systems [31].

The method of calculating aggregate volume is straight forward. Three video cameras are mounted from three orthogonal directions: front, side, and top. The images acquired from these three views provide some capability to reconstruct the 3-D shape of the particle needed for volume computation. The particle is confined in the smallest box whose sides are found to be the smallest rectangle that includes the particle projected area in that viewing direction. Those pixels, called solid pixels, can be found readily which belong to the particle body from all three viewing directions. All the cubes made up of the solid pixel are summed up, and calibrated to cubic millimeters. Hence, the volume of the particle under study is obtained. However, it is fairly easy to envision shapes for which even three orthogonal views are taken would not be sufficient to accurately evaluate particle volume [33]. The particle touching and overlapping problem is avoided because all particles fall one at a time onto a belt that is in motion. Though the system performance is expected to give improved accuracy, it is more time consuming since the particle is processed individually on a conveying belt.

The Micrometrics Corporation sells a device similar in design to the VDG 40. The Optisizer PSDA ${ }^{\mathrm{TM}}$ uses a vibrating feeder and a CCD camera to capture a 2-D image of particles from 40 micrometers to "greater than $10 \mathrm{~mm}$ " [36]. This device is more suited to pharmaceutical environments than construction work, however. No mention of particle shape analysis is provided, nor are statistics on the sieving accuracy of the machine. 
In addition to these devices, articles related to optical sieving or particle size and shape evaluation have been published in the technical literature by a variety of authors. Parkin, et al published a proposal for a laser based aggregate scanning device in 1995, but no further references to their system have been found [4].

\subsection{Separation of Overlapping Image Objects}

Bennamoun and Bouashash [3] introduced a segmentation method based on the successful completion of robust edge detection. The segmentation algorithm begins with extracting the convex dominant points (CDP), then use these CDP's for the part segmentation by simultaneously moving each of them normal to the edge contour until one CDP touches another point. Next the initial locations of CDP's are joined to the touched points. This process is repeated until the whole object has been segmented into constituent parts. The segmented parts are then isolated and modeled by superquadratics with varying parameters for recognition purposes.

A templating approach for separating the touching and overlapping spots is introduced by Noordmans and Smeulders [14]. The technique consists of two phases: detection phase and characterization phase. In the detection phase, all image positions are matched to a spot model with predefined parameter vector and coordinate. The optimal match is given by the specific value of parameter vector that results in a minimal match error. Following the detection phase is the characterization phase. The primary purpose of this phase is to further reduce the match error. Detecting two overlapping spots is based first on the observation of two major match errors, then extracting the local image. After removing one neighboring spot, the first spot is optimally matched with the 
model using numerical minimization procedure. By the same method, the second spot can be detected and characterized. This way, two overlapping spots are thus detected and characterized independently.

In morphological image processing, the watershed detection approach proves to be an efficient way of segmenting gray scale images or binary images. Vincent [28] provides a faster, more efficient algorithm than those introduced previously to detect the watershed for a gray toned image. The basic principle behind this technique is that the whole gray scale image under study is considered as a topographic surface. This surface is made up of basins (valleys) and mountains. The watershed algorithm computes the dividing lines between the different "catchment basins", which become regions or objects in the image. In the case of a binary image, the effect is to separate touching or overlapping particles. A modification of this approach was developed for use in this research.

\subsection{Particle Passage Probability in Sieving}

Most probabilistic studies of particle-passage through a sieve relate the probability of passage to sieve aperture size and particle shape. Bocoum [41] reviewed some probability theory in sieving. In summary, the particle-passing probability through a screen depends on the following aspects:

1) Three dimensional shape of the screen, and

2) Its relative size to the size of the particle

3) The percentage of open area on the screen surface.

4) Screen surface roughness. 
5) The speed of the particle upon impact.

The primary studies of particle-passing probability were developed for particles of three geometric shapes: spheres, ellipsoids, and cylinders. For these three shapes, the theoretic passing probability was reviewed in Bocoum's paper. However, no conclusive information was presented for the irregularly shaped particles passing through the square sieve aperture.

\subsection{Object Shape}

Particle shape is an important factor in particle handling and product quality control. Since the particle shape influences how particles flow, react, sinter, break, agglomerate, and fluidize, numerous shape characterization techniques have been demonstrated over the last decades [8].

Particle shape analysis can be divided into two broad categories: behavior analysis and image analysis [9]. Most image analysis techniques rely on examining a two-dimensional image silhouette of the particle shape. Analysis of particle image can be conducted in either a microscopic or macrosopic manner. The microscopic method is used to describe the particle's relatively subtle change on the surface such as angularity and roughness. The macroscopic method, on the other hand, is more general in the sense of describing particle shape. This approach usually provides information in 2-D image about particle characterized shape such as triangle, four-sided, etc.

In a microscopic shape study, Clark made some explorations of fractal analysis

[7]. Fractal analysis originates from the fact that the perimeter of the silhouette edge is dependent on the step length with which it is measured. The small detailed features on 
edge can be taken into account with step length small enough, while taking large step length will ignore some delicate characteristics of the edge. The measured perimeter is increased if the step length used is decreased, yielding the notion of "fractal dimension" that can be used to describe particle ruggedness over a range of scale. A logarithmic plot of perimeter against step length produces a curve with negative slope. Steepness of the curve slope is used as a descriptor indicating the extent of the ruggedness of that particle silhouette. Fractal dimension shows the general degree of particle ruggedness, but does not provide general geometric shape information.

In a more macroscopic approach, Clark, and Reilly introduced a novel approach called polygonal harmonics to describe the particle shape $[9,10]$. A starting point is selected on the edge of the particle, then a pair of dividers is set at some distance and used to find another point on the curve. Sequential points on the edge are found in the same manner by marching along the edge of the particle. The procedure is similar in this regard to a structured walk to find fractal dimension as mentioned previously. The walk continues past the first starting point, traversing the silhouette edge over and over again. Eventually a polygon is formed with a fixed dividing step length within the shape. Different step lengths produce different polygons for the same particle shape. Harmonic persistence is defined as the ratio of the largest step length to the smallest step length yielding that particular polygon. High harmonic persistence is an indicator of general particle shape.

This approach has shown some satisfactory results. However, in general it does not guarantee that a particular polygon exists for a given shape silhouette. Repetition of computation using different step lengths to find harmonics persistence is needed for each 
particle [10]. Moreover, the persistences are not unique to each analytical shape, nor can the shape be reconstructed using the persistences [12].

Fitting approaches have been found in a variety of literature. In the papers by Bennamoun and Bosshash [3], Rosin and West [6], object shapes are described by fitting the object edge silhouette with superellipses. Each superellipse is described by three parameters: major and minor axis, and shape factor. One superellipse can be found to be the best fit to the shape in question by minimizing the Eucidean distance between the point on the superellipse and the point on the edge silhouette. Using the three identified parameters of this particular superellipse, the shape can thus described. The advantage of this technique is that a superellipse can represent a wide variety of shapes. with a small number of parameters.

Another template matching is to fit the object edge silhouette with a square instead of a superellipse. The side length of the square is used as the descriptor for the shape to show how square-like or rhombic-like that particle is. The best fitting square is found by minimizing the area error between the square and the particle of interest. The merit of this technique is that only a few parameters are necessary for describing the shape in question. However, neither the superellipse nor the square fitting approach can accurately represent shapes with odd numbers of sides. For instance, a triangle shaped particle can never be fitted well by either the superellipse or square. Moreover, both techniques are computationally intensive. Algorithm convergence is not always guaranteed. This disadvantage is even more severe when applied to a large number of particles in a single image. 
A set of descriptors called "invariant moments" was studied [2]. Invariant moments are derived using the central moments of the image shape. Because of the relation of central moments with the regular moments, and the uniqueness of these regular moments relating to a certain image function, the chance that different shapes have the same or even close invariant moments is small. Therefore, invariant moments can be utilized to describe the shape features.

All the above shape descriptors share the same merit: they are translationinvariant, rotation-invariant, and scale change invariant. These attributes are necessary for shape feature classification in a multi-object situation. The negative aspect about using the above techniques is the computational intensity.

Fourier analysis has long successfully employed on smooth, rounded particles. In Fourier analysis, the edge is described by expressing the radius from the centroid of the shape as a function of the swept angle, using a Fourier series. For instance, the second coefficient gives an implication of aspect ratio, and the third coefficient indicates triangularity, and so on. Particle shapes can be compared in a n-dimensional space composed of the $n$ orthogonal Fourier coefficients [13]. The well-known weakness of Fourier analysis lies in the fact that it does not deal efficiently with highly reentrant shapes.

\subsection{Object Size}

Size and shape issues are usually intertwined in image processing problems. Various specifications for object size description have been found in technical reports: for objects of regular shapes such as squares and circles, side length and diameter are used 
respectively to define sizes. For irregularly shaped objects, major and minor dimensions are well-defined measures, although they do not guarantee uniqueness of shape description. Size, defined by the object's projected area, can be found explicitly and implicitly described in various papers. In Rosin and West [6], it can be inferred that the size is defined by the parameters of the superellipses, and is also represented by its area.

Size definition is problem-oriented. In the Superpave manual [1], the size of aggregate is measured by its sieve size. Fig. 2.1 illustrates how a particle's minor diameter corresponds to the square sieve opening size.

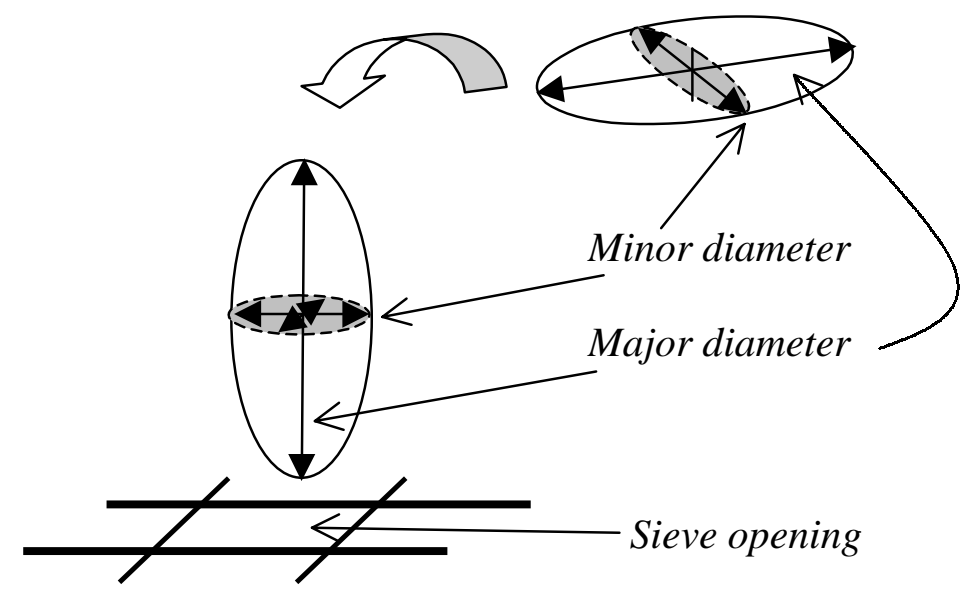

Figure 2.1 Aggregate Size by Superpave

The correlation between a particle's minor diameter and the sieve opening size is rather complex, sensitive to the actual size, particle shape, surface roughness, orientation and the interaction with all the touching particles at the moment it is about to pass the opening, and very sensitive to the amplitude and waveform of vibration. It becomes more complicated to quantitatively analyze and simulate the mechanical sieving process. 


\section{LABORATORY SET-UP AND MEASUREMENT CALIBRATION}

\subsection{Introduction}

A video camera translates light levels focused on the image plane into electronic signals which can be transmitted and reproduced on a monitor set. The most common type of video camera uses a charge coupled device (CCD) chip to translate the light into electrical signals. The CCD chip is actually a grid of tiny individual light measuring devices which break the scene up into individual picture elements, or pixels. The camera used for this research breaks each scene into an array of 512 pixels wide and 484 pixels high.

To process these signals using a computer, the light level represented by the video signal must be digitized by translating the signals into a series of numbers that the computer can manipulate. This is implemented by a frame grabber board, which performs very fast analog-to-digital conversion on the electronic signal for the camera. As a result, a grid (matrix) of numbers ranging from 0 to 255 , with one number for each pixel, is formed. Low numbers represent dark parts of the image and high numbers represent bright parts of the image.

To optically sieve the particles, it is necessary to translate the pixel measurements into standard dimensions of millimeters. Pixels are in general not square, and so a unit of one pixel represents a different length in the $x$ direction than it does in the $y$ direction. In addition, the object is projected optically onto a CCD array. This causes the size of the image to depend not only on the size of the object but also on its distance from the camera, and on the focal length of the lens used to project the image onto the CCD 
sensor. Therefore, a scale of $\mathrm{mm} /$ pixel needs to be determined before any useful image analysis takes place.

\subsection{Hardware Set-up and Operating Condition}

The laboratory consists of a video camera housed in a curtained enclosure to allow control of the lighting conditions, a computer with a frame grabber card, a box with translucent cover to backlight the aggregates, and miscellaneous equipment for scene illumination, positioning the camera, measuring the light level, etc. The photo in Fig. 3.1 demonstrates the actual lay-out of the hardware components. The interior of the wall was painted black to reduce light reflection. All the components involved are numbered and illustrated in Fig. 3.2. Other associated devices, such as mechanical sieves, laboratory balance, were used in the Asphalt Pavement Laboratory in the Civil and Environment Engineering Department at West Virginia University.

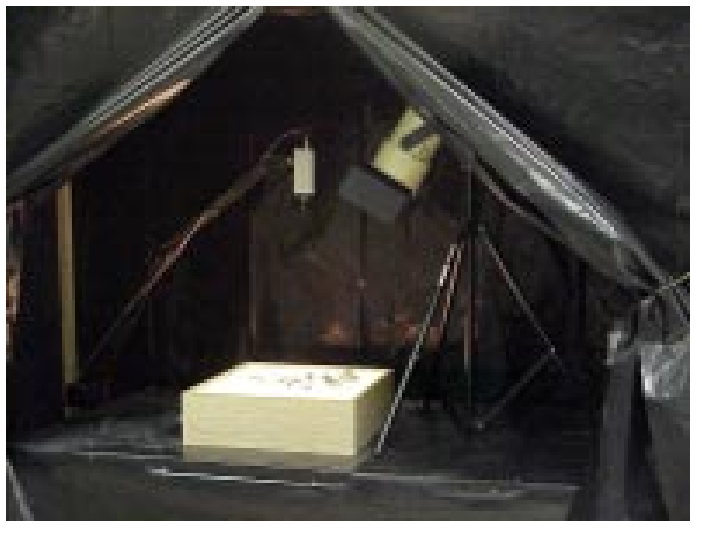

(a) Image capturing set

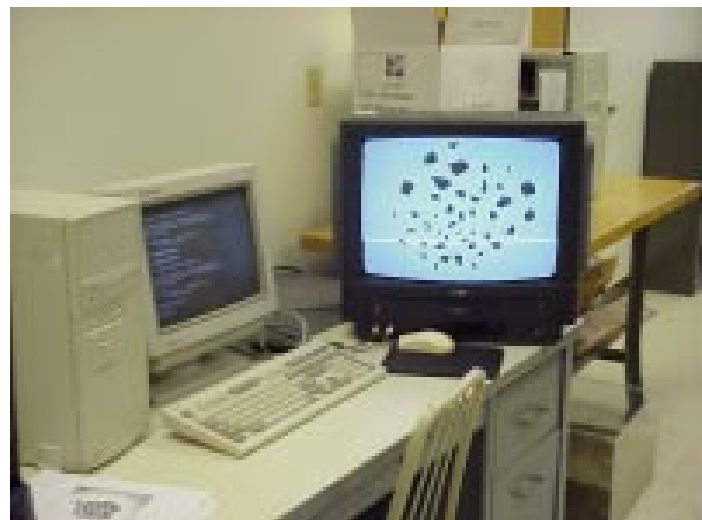

(b) Image processing set

Figure 3.1 Photo of Lab 


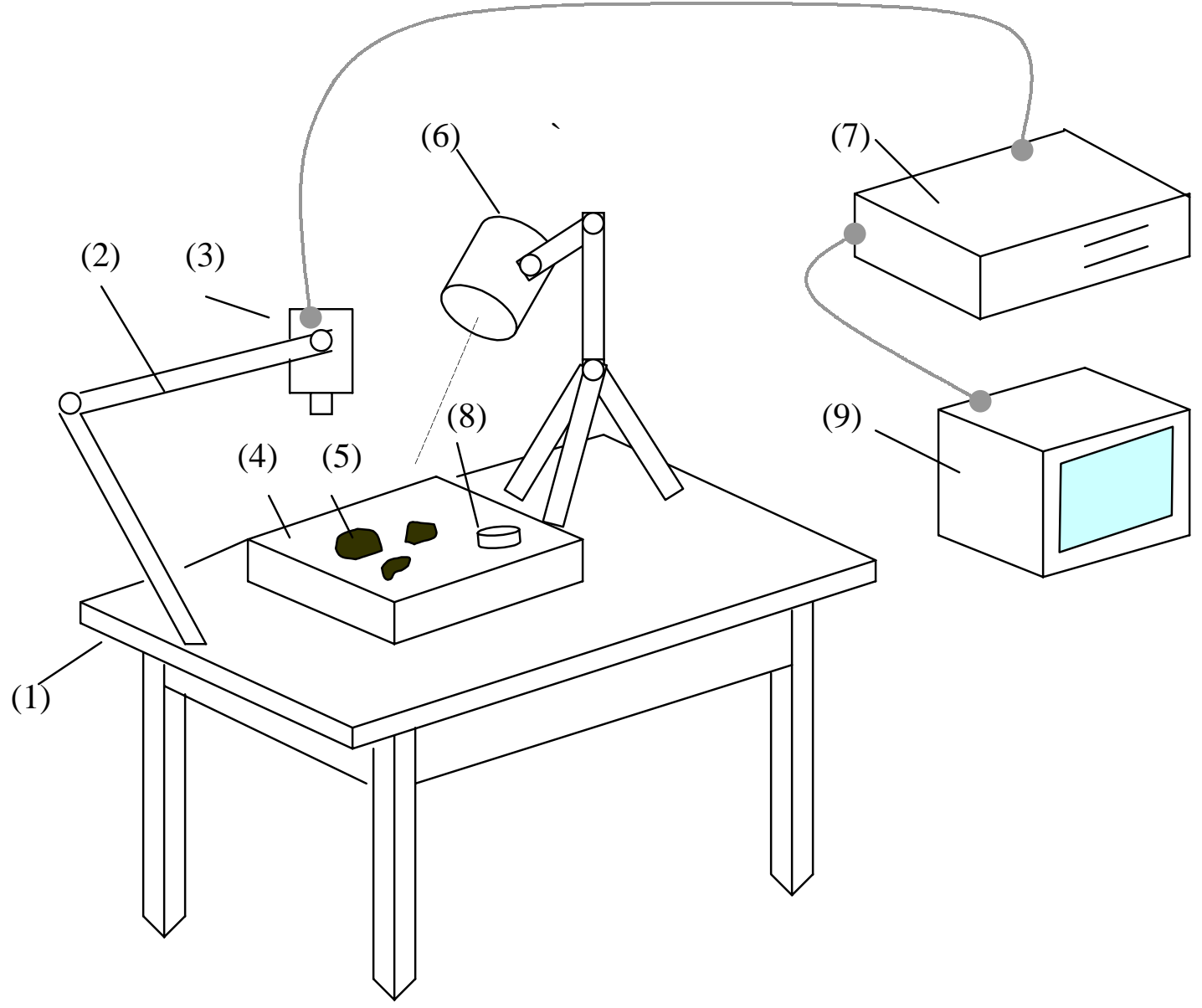

\subsection{Lab Equipment Lay-out}

Referring to Fig. 3.2, the representation of the numbered item is as follows:

(1) Stationary table.

(2) Pan-tilt device with 6-degrees of freedom.

(3) Video camera.

(4) High contrast lighting box.

(5) Aggregate particles.

(6) Photographic strobe light for oblique lighting. 
(7) Personal computer with frame grabber.

(8) Light intensity meter.

(9) Monitor.

The operating specifications for image capturing using the lighting box are given in the table below:

Table 3.1 Operating Condition

\begin{tabular}{|l|c|l|c|}
\hline $\begin{array}{l}\text { Camera } \\
\text { Distance from } \\
\text { background }\end{array}$ & 20 inches & $\begin{array}{l}\text { Camera } \\
\text { aperture }\end{array}$ & 6 \\
\hline $\begin{array}{l}\text { Camera } \\
\text { Focus }\end{array}$ & 20 inches & $\begin{array}{l}\text { Ambient lighting } \\
\text { intensity }\end{array}$ & 12 LUX \\
\hline $\begin{array}{l}\text { Camera } \\
\text { Shutter speed }\end{array}$ & $1 / 125 \mathrm{Sec}$ & & \\
\hline
\end{tabular}

\subsection{Image Acquisition}

In order to properly "sieve" the aggregates, it is necessary to distinguish one particle from another in the video image. The gray scale video images that are most commonly seen, seem simple to the human observer to see where object boundaries are, while the information presented to the computer from these images is nothing more than a large grid of numbers. To detect these boundaries, most approaches involve some sort of gradient detection - looking for places in the image where there are rapid changes from light to dark or vice versa. Some of the object boundaries are clearly defined by contrast between the background and the existence of object shadows. But if imaged objects such as mineral particles overlap, the contrast between two particles may not be so distinct. In addition, the existence of ridges or corners on the particles can produce high-contrast edges which are not true particle boundaries in the 2-D sense. 
Various edge-finding algorithms and lighting angles were explored to find a method that would reliably detect the boundary of each particle. The simplest and most common edge detectors are first-order high-pass filters based on the Sobel Operator or variants thereof [2]. These filters are highly sensitive to noise and directional in nature, performing best on edges that are either vertical or horizontal. Sobel filters combined with top lighting are also prone to including unwanted edges, such as those resulting from corners or shadows on the top surface of the particle.

To eliminate interior edges, a small light table was constructed for backlighting the aggregates. This lighting method produces extremely high contrast images with welldefined edges. In the phase of obtaining image data, objects are backlit to obtain sharply distinctive edges from black images on a white background, as illustrated in Fig. 3.3. Once the image has been captured under the operating conditions specified previously, each image is stored as a set of $x-y$ points. Image processing and analysis are performed on these sets.

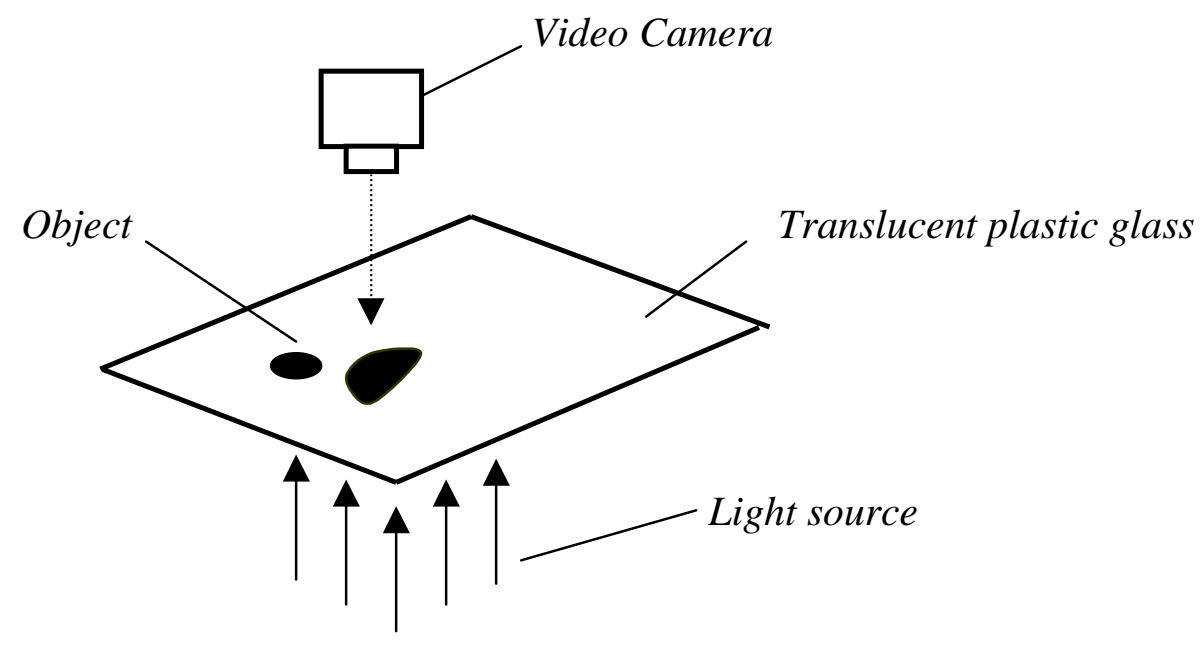

Figure 3.3 Image Capturing with Backlighting 
Fig. 3.4 illustrates the procedure related to image analysis and processing..

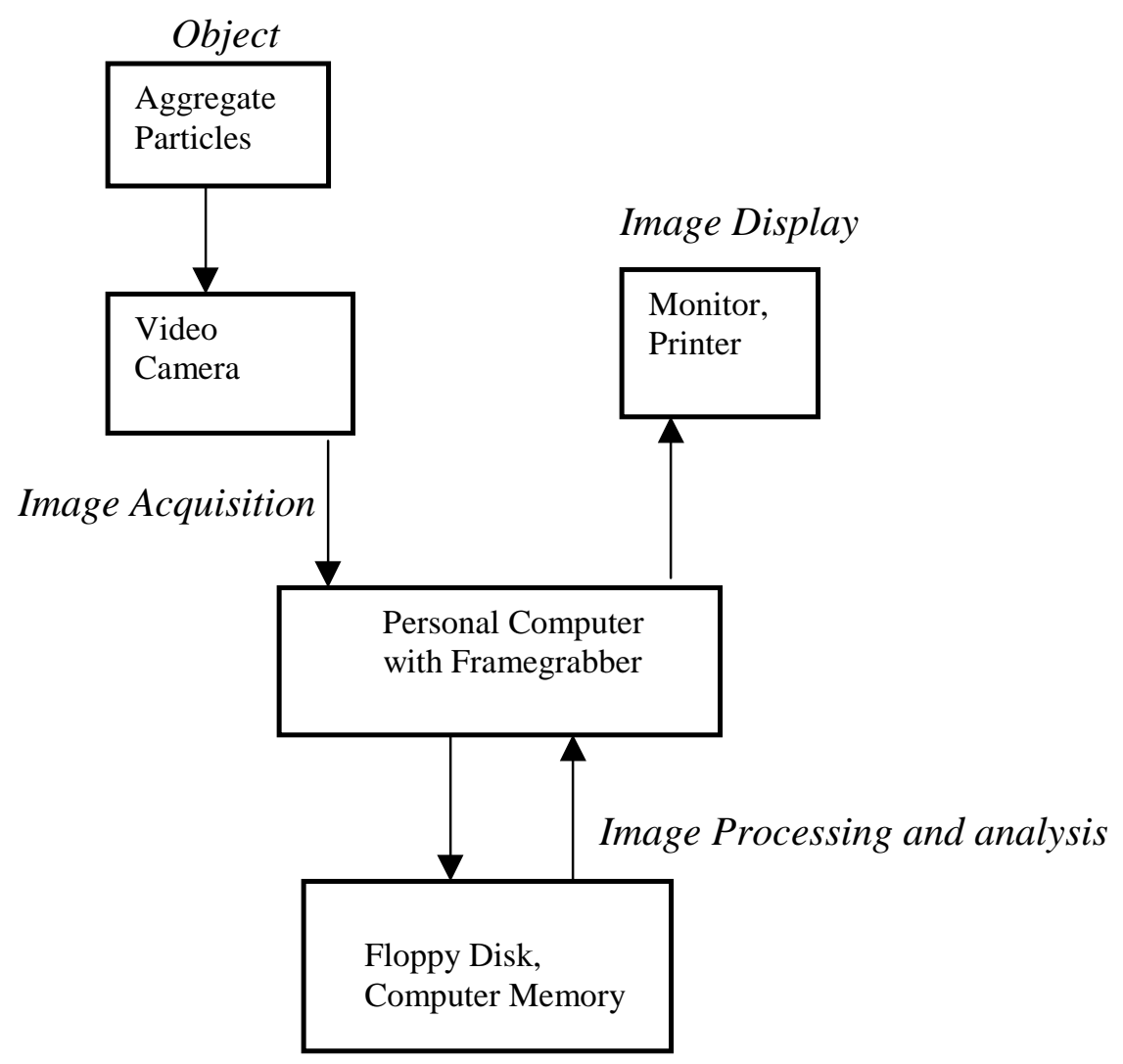

Figure 3.4 Image Acquisition and Processing System

\subsection{Image Measurement Calibration}

The measuring unit for the image is the number of pixels. For example, the image area for a given particle might be 100 pixels, and the circumference length might be 50 pixels, etc. The actual measuring unit is millimeters, thus a conversion from pixels to millimeters is required. In other words, the scale of $\mathrm{mm} /$ pixel needs being determined. 


\subsubsection{Sample for Calibration}

Three types of sample circles were found using penny, nickel, and quarter. Their diameters are $19.05 \mathrm{~mm}, 21.12 \mathrm{~mm}, 24.20 \mathrm{~mm}$, respectively. The corresponding areas are $285.02 \mathrm{~mm}^{2}, 350.33 \mathrm{~mm}^{2}$, and $459.96 \mathrm{~mm}^{2}$. The distance between the camera and the imaging background is 20 inches, set constant for all images. The parameters of the camera such as shutter speed and aperture were unchanged during the imaging process.

Fig. 3.5 shows the samples used for calibration.

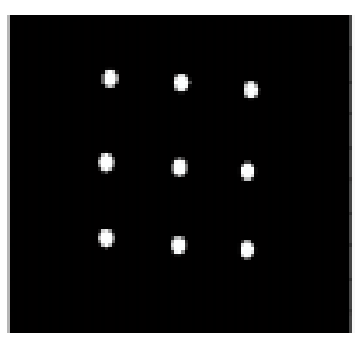

(a) Pennies

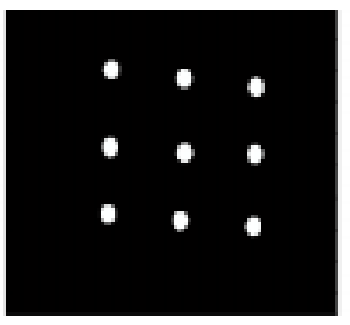

(b) Nickels

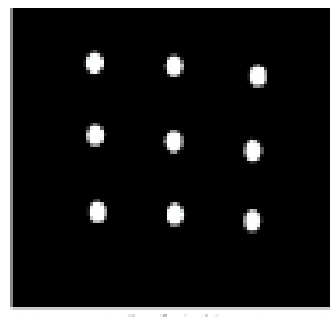

(c) Quarters

Figure 3.5 Samples for Calibration

\subsubsection{Finding Pixel Number}

Fig. 3.6 shows the pixel number for the diameter of the three sample circles. Note that the horizontal and vertical numbers are different. They are obtained by scanning the image in two orthogonal directions. Note also that although 9 coins are used for each type of circle, the plot shows that some resultant pixel numbers are coincident with each other. 


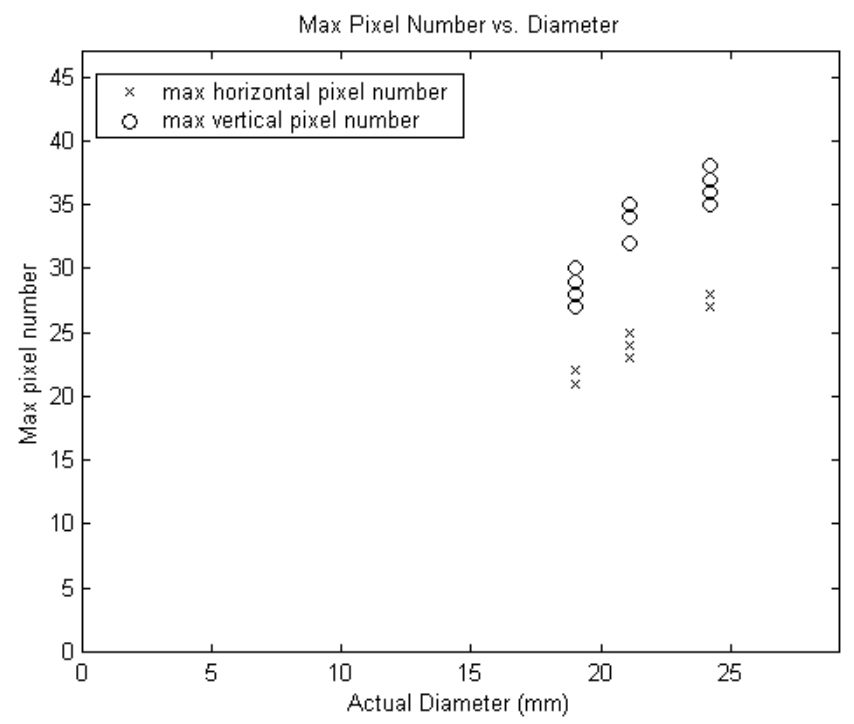

Figure 3.6 Maximum Pixel Number

Using least squares curve fitting, both the horizontal and vertical pixel data points can be fitted with a straight line, which is forced to go through the origin. Fig. 3.7 illustrates the result.

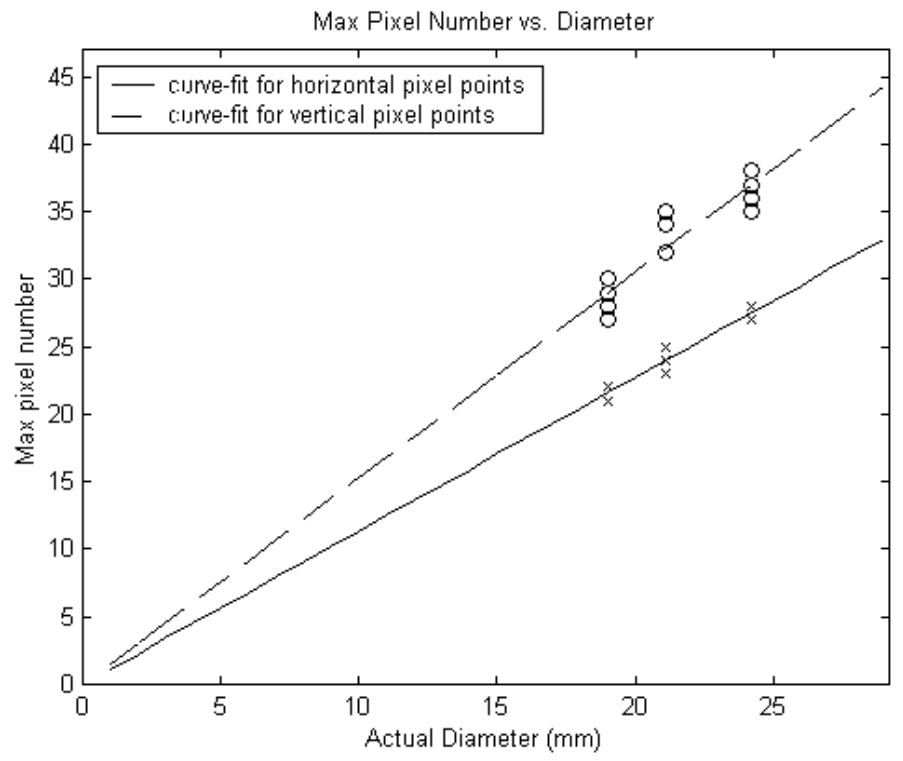

Figure 3.7 Data Curve Fitting 
The reciprocal of the slope of each straight line is taken as the desired scaling factor of $\mathrm{mm} /$ pixel . The results are: $0.8802 \mathrm{~mm} /$ pixel in the horizontal direction, and $0.6551 \mathrm{~mm} /$ pixel in the vertical direction.

\subsubsection{Area Correction}

Prior to calibration, the object area is measured in number of pixels. Using the scale factors obtained previously, the measured area in terms of square millimeters can be acquired. Fig. 3.8 shows the plot of the findings against the corresponding actual areas.

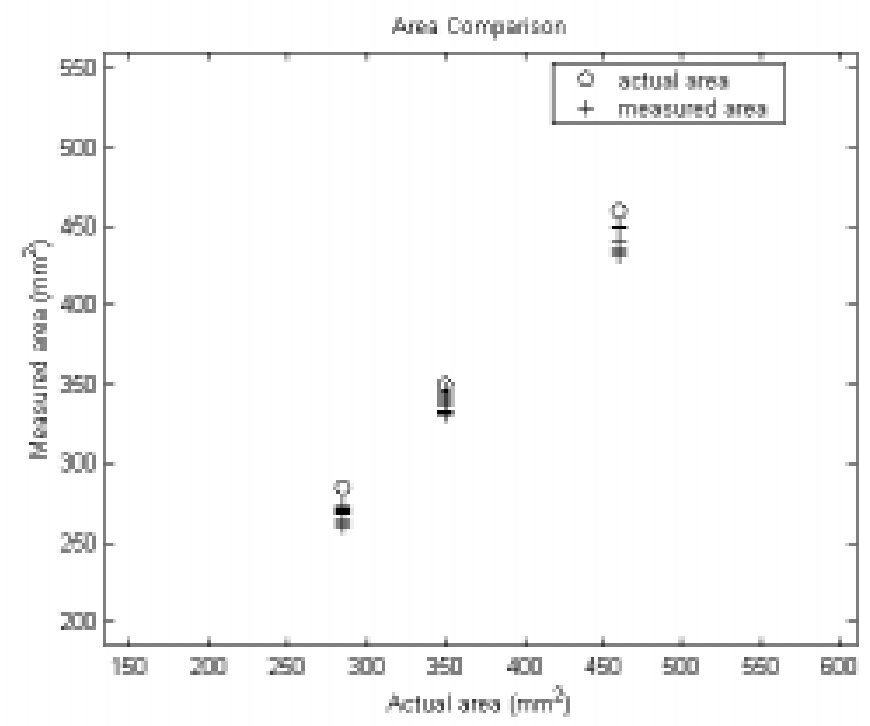

Figure 3.8 Measured and Actual Area

The measured area data points are curve fitted by a straight line using least square method, as shown in Fig. 3.9. Note that the value on the abscissa is actual area, and this leads to the relation between the actual area and the measured area. This function was found to be 


$$
A_{t}=\frac{\left(A_{m}+7.9335\right)}{0.9742}
$$

Where

$$
\begin{aligned}
& A_{t}: \text { actual area }\left(\mathrm{mm}^{2}\right) . \\
& A_{m}: \text { measured area }\left(\mathrm{mm}^{2}\right) .
\end{aligned}
$$

The above transformation is necessary because there are "dead areas" in the image between pixels, so simply multiplying the two scaling factors leads to incorrect results.

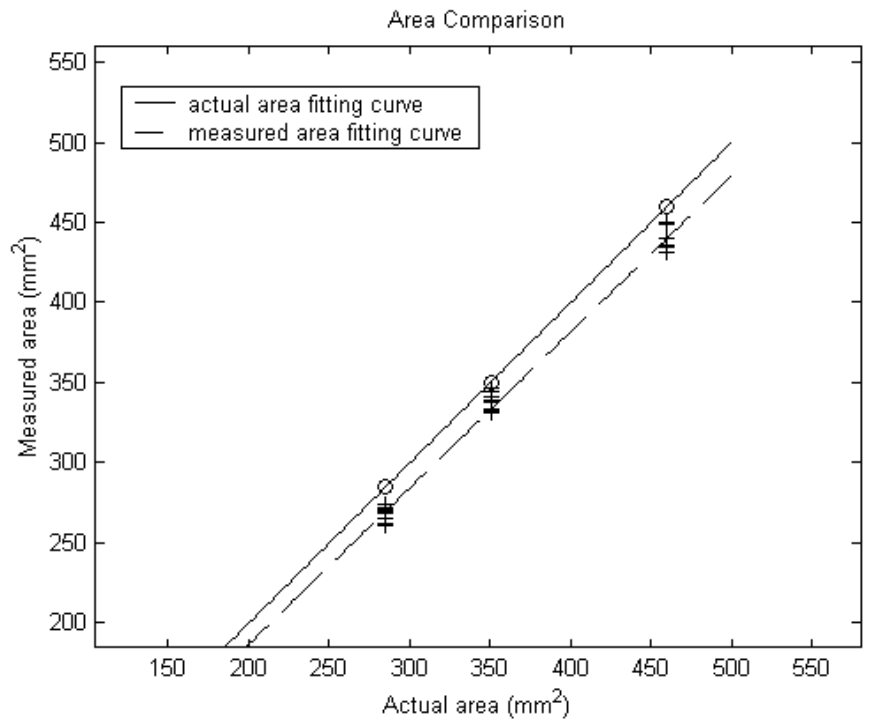

Figure 3.9 Curve Fitting for Measured Area

As illustrated in Fig. 3.10, the measured areas are much closer to the corresponding actual areas. As a result, the accuracy of the measured areas is improved. 


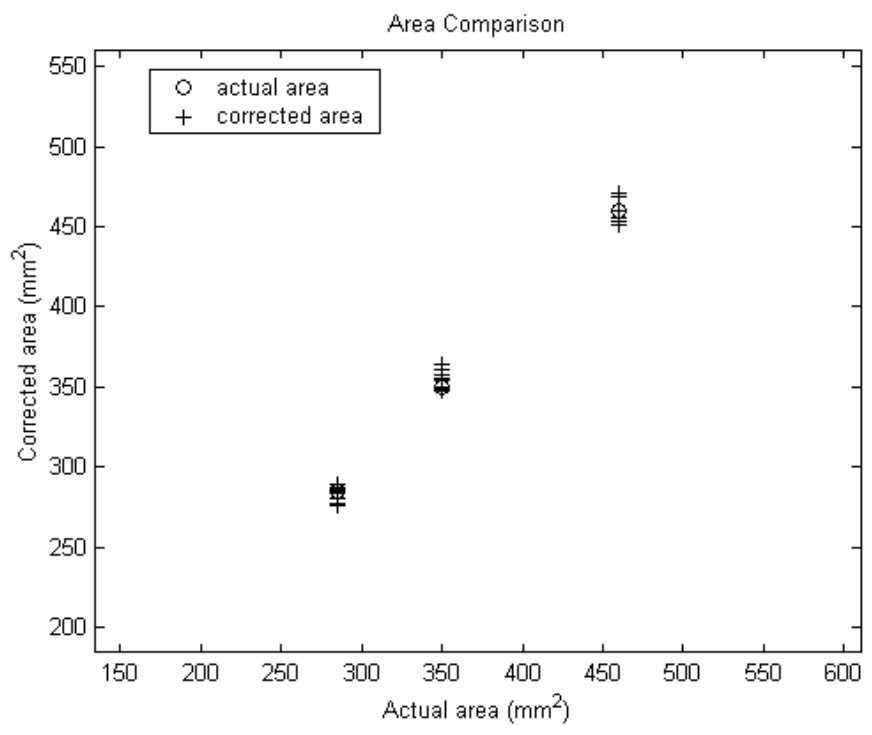

\section{Figure 3.10 Improvement of Measured Area}

The improvement in the measured areas can be demonstrated by observing their absolute percent error before and after using Eqn. (3.1). The absolute percent error for three circle samples are shown in Fig. 3.11.

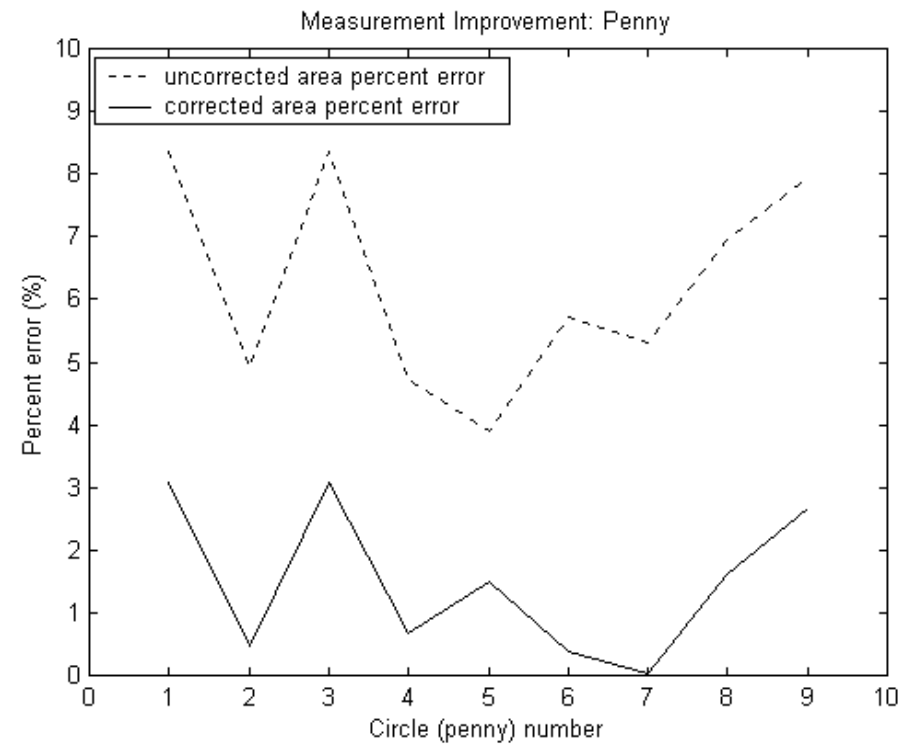

(a) absolute percent error for pennies 


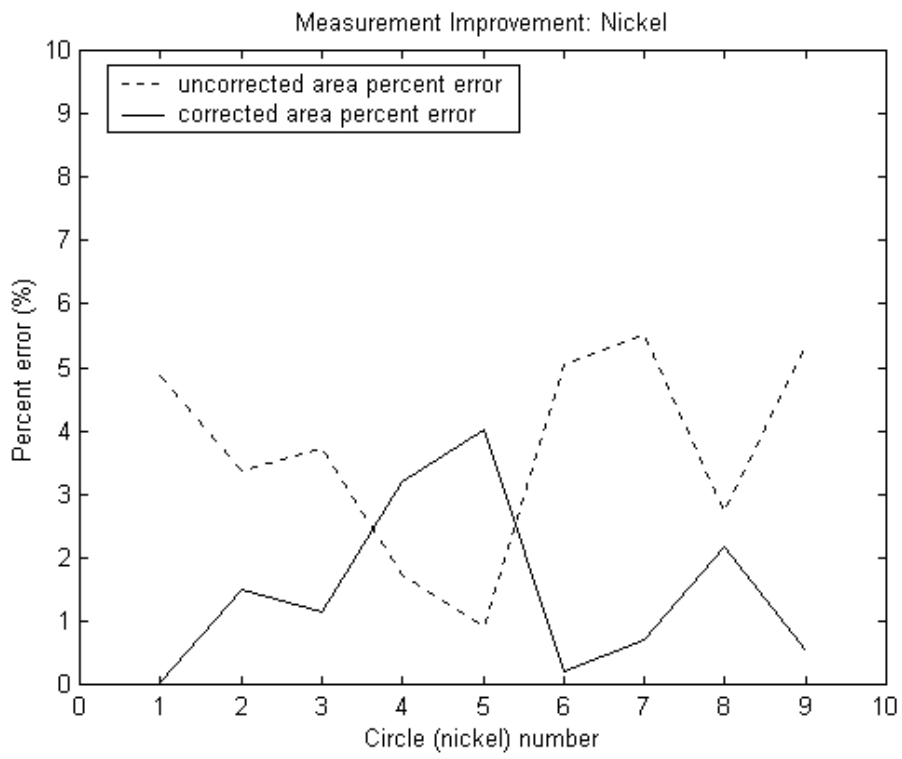

(b) absolute percent error for nickels

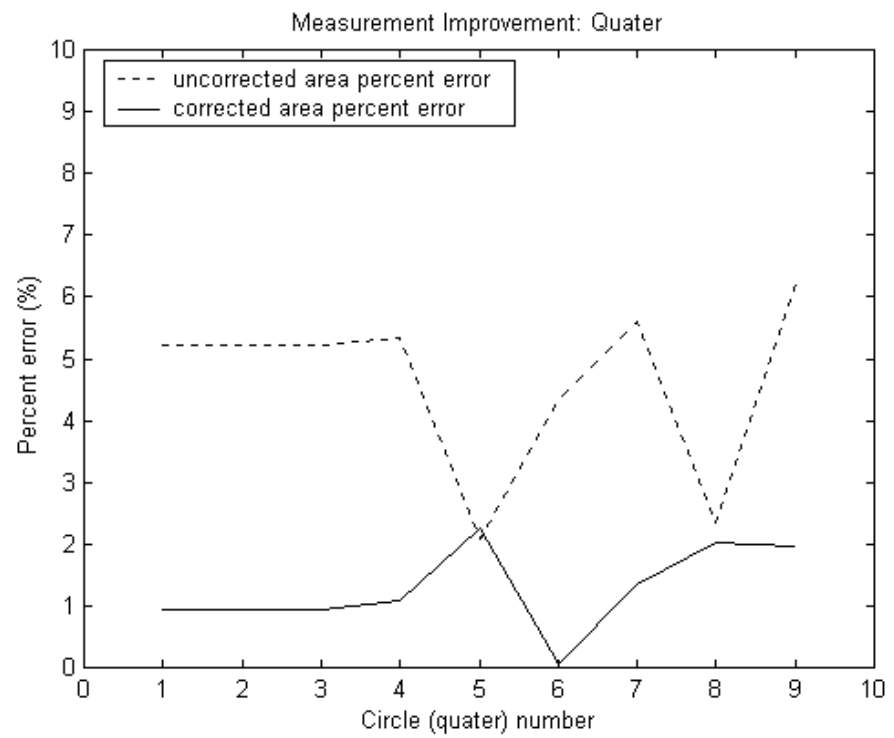

(c) absolute percent error for quarters

Figure 3.11 Absolute Percent Error Improvement 


\section{IMAGE PROCESSING AND ANALYSIS}

\subsection{Introduction}

For each imaged object, that is, a non-touching-overlapping particle, the size and shape as well as some other parameters must be computed for the particle volume estimation and optical sieving purposes. Based upon the binary images - all particles are white and background is black, the area, size, shape, and some other related statistics are calculated. Image preprocessing includes binary conversion, edge detection, and separation of the touching and overlapping particle shapes. By image analysis herein, it

means finding the particle shape centroid, area, major and minor diameters, identifying shapes, and computing all the needed statistics of the particle in question.

Solution for finding above measurements is summarized in actual research sequence as follows:

1) Binary image conversion.

2) Image capturing and seeding

3) Edge detection, region growing and particle projected area calculation.

4) Centroid location.

5) Major and minor diameter computation.

6) Particle profile shape characterization.

Successful completion of the image preprocessing and analysis paves the way to establishing a mathematical model to estimate the volume of particle, and ultimately, to obtain the particle size distribution through a sieving correlation process. 


\subsection{General Description}

Fig. 4.1 shows images of four simulated particles. Sub-figure (a) simulates the binary image that is the result of image processing, while (b) shows the completion of the analysis to it. Once the particles have been converted to binary images and separated, analysis starts with horizontal scanning and tracking the edge of each particle. During the edge following, edge points (or pixels) are stored in an ordered list, and the interior points are counted to compute the projected area of the particle. Calibrated scaling factors are used to transform pixel numbers into dimensional measurements. The centroid of the particle is calculated during the scanning process, and the pixels belonging to the particle under consideration are labeled so they can be eliminated from future scans.

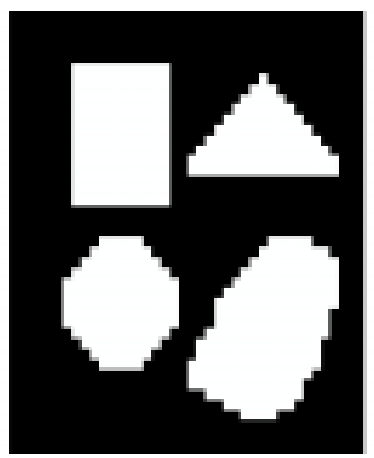

(a)

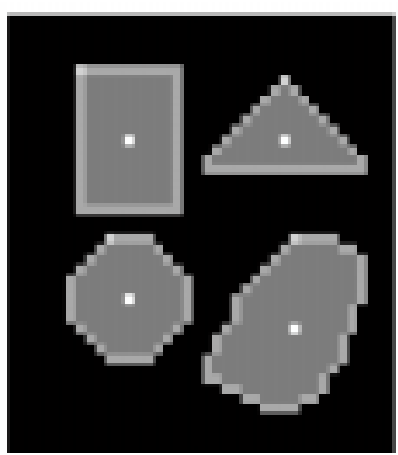

(b)

\section{Figure 4.1 Simulated Particles with Centroid,Edges} and Interior Points Labeled

Once this process is done, the list of edge points is sampled and the Euclidean distance from the centroid to each of the edge points is computed. Because particle sizes vary significantly, the sampling algorithm may be set up to choose an adequate number of points from the edge to yield a good description of the particle silhouette in oder to 
minimize the amount of computation required per particle. In this research, each edge point is sampled. This sampling method results in samples at uneven intervals of the polar angle $\theta$ from the centroid, but avoids the time-consuming search for points satisfying the angle interval criterion and the repeated calculation of the inverse tangent function.

To characterize the particle's profile shape, the "edge signature" is constructed. A signature gives the distance between each edge point and the centroid, or, the radius at each edge point, so the information about the particle's shape can be stored in the signature function. To eliminate the noise, the signature function is fitted by a polynomial. Since the order of the polynomial is lower than the number of signature points, significant smoothing of the curve occurs, yet the polynomial is complex enough to track even relatively jagged particle boundaries accurately. The maxima and minima of the polynomial can be computed, as can the sum of squared errors between the actual signature points and the fitted curve. In general, maxima of the polynomial corespond to the vertices of the particle, and the "significant" minima are often created by flat faces, as demonstrated in Fig. 4.2. 


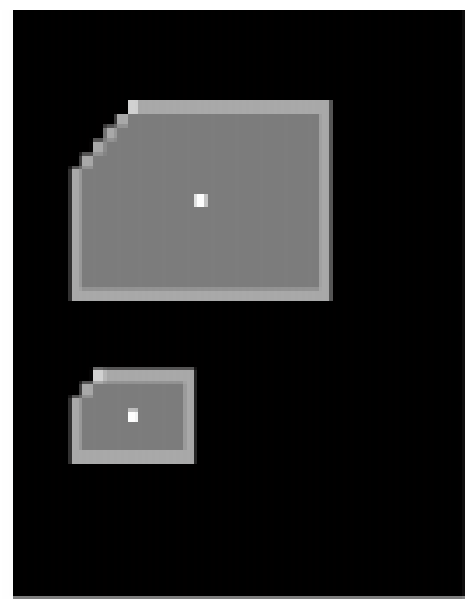

(a) two similar shapes

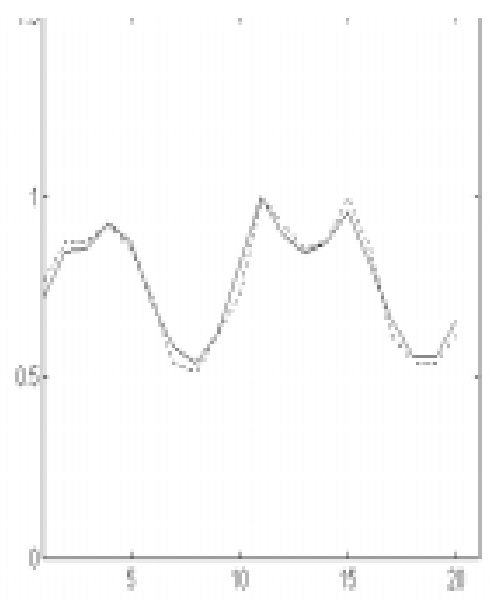

(b) normalized signatures

Figure 4.2 Shapes and Corresponding Signatures

One important further use of the edge points is made. To correlate particle size to sieve size, it is critical to measure a particle's dimension. For spherical particles the correlation is easy, but for crushed aggregates it becomes very difficult. The solution is to compute a covariance matrix from the edge points, and then to use the two eigenvectors of that matrix to compute the "major" and "minor" diameters of the particle. These dimensions are usually close to but not always coincident with the actual maximum and minimum dimensions of the particle shape. Major and minor diameters are used to compute the elongation of a particle. Note that the relationship between actual particle dimension and sieve size is very complex, involving quantization effects and sensitivity to particle shape and orientation. In this work, the minor diameter is used to correlate the sieve size. 


\subsection{Binary Image Conversion}

Edge detection can be carried out much more easily on a binary image than a gray scaled one. Computations involved in finding particle projected area, locating the centroid, characterizing profile shape are all originated from edge detection.

A high contrast image can be obtained using the backlighting box to silhouette aggregates spread on its surface. In Fig. 4.3 (a), two pieces of aggregate were placed in the scene and digitized on a $484 \times 512$ image matrix. Note that the shadow is present because backlighting is not used. To make the problem clear and simple, the two rocks were separated. Fig. 4.3 (b) shows the image captured using the backlighting box. In order to efficiently extract geometric information from the image, thresholding is taken to reduce unnecessary gray-scale variation, thus a binary image is obtained, as depicted in Fig. 4.3 (c) and (d).

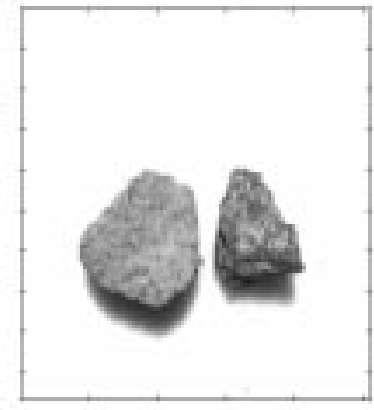

(a)

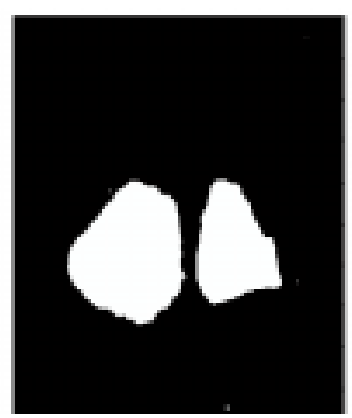

(c)

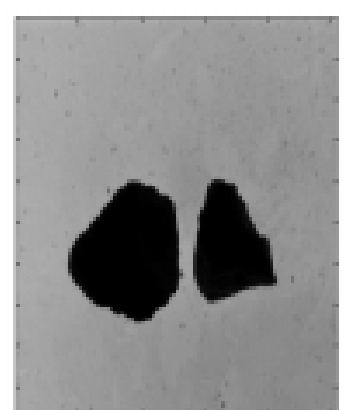

(b)

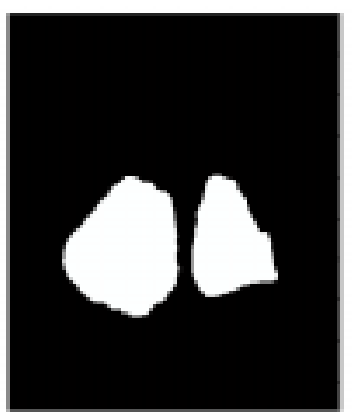

(d)

Figure 4.3 Binary Image Conversion 
Fig. (a): Image captured without using lighting box. Particle edges can be difficult to distinguish from shadows.

Fig. (b): Image captured using lighting box. Conversion to binary image is carried out on this image.

Fig. (c) (d): binary image obtained, before and after removal of small speckle noise. The speckle noises can be caused by both insignificant tiny particles and unclean camera lens. Checking the spot size experimentally can remove them.

\subsection{Object Detection and Seeding}

The whole object detection and seeding procedure is featured by "piecewise" processing in terms of particle number order. The object of interest is processed by the algorithm designed for acquiring all the needed measurements.

Particle detection is performed by taking advantage of horizontal scanning over the binary image. An object is detected when the first edge point belonging to that specific object is encountered by the scan moving point (SMP), which is travelling horizontally from left to right within the object. This first encountered point works also as a seeding point, or simply seed. A seed is always located at the top-most-then-leftmost (TMLM) position on the detected Object. Object detection and seeding is completed simultaneously. The seed is used as the starting point from which the region growing will take place. In Fig. 4.4, the brightest point on the object indicates that the object is detected and seeded. Note that for demonstration purposes, the image presented here is not a binary one, but rather an intensity one with gray scale 64. This is for 
showing up the seed location. In fact, pixel labeling is imbedded throughout the algorithm for various processing purposes.

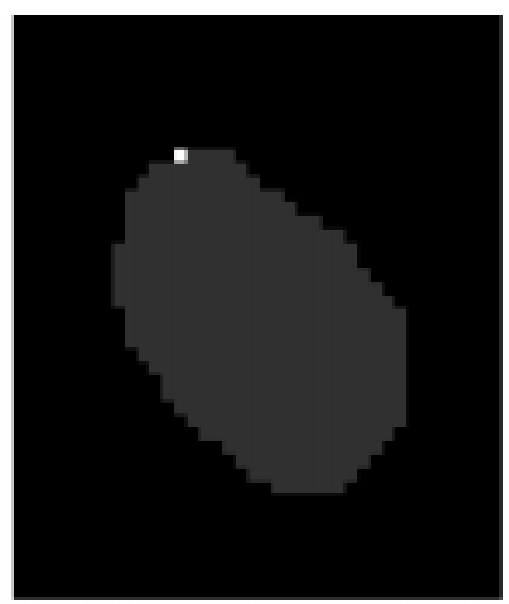

Cbject Captuing. Gray Scale: 64

\section{Figure 4.4 Object Detected and Seeded}

In the multi-object case, an object whose TMLM edge point is also in the topmost and left-most position in the image matrix, will be detected and seeded first, since the SMP is traveling rightward, and the scan line is moving downward. Once the object's last pixel has been encountered by SMP, this object is isolated from all other objects, processed and would-be-processed alike, in order to avoid being re-encountered by the SMP. The detail about isolation is given in the later section. The processed object can also be considered as having been converted to the background, and it will be ignored by the SMP. The next candidate object to be detected and seeded is the one whose TMLM edge point satisfies the position conditions for detection priority. Fig. 4.5 shows four simulated overlapped but separated objects. Fig. 4.6 illustrates the sequence of detection and seeding for these four objects. 


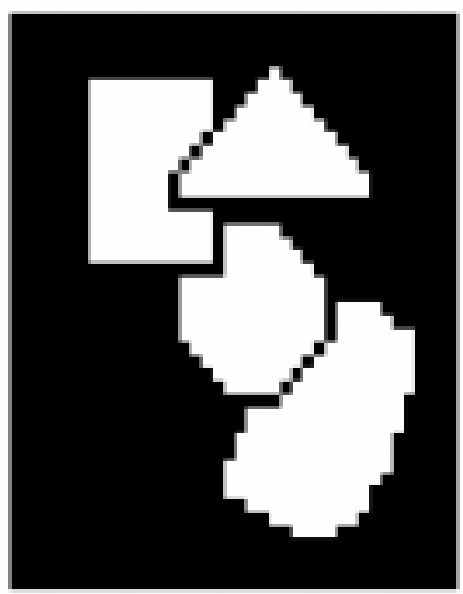

The Binayimage

Figure 4.5 Four Simulated Objects

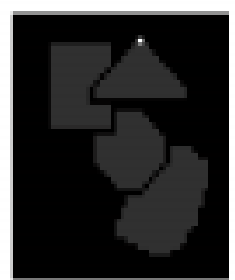

(a)

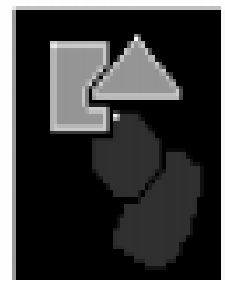

(e)

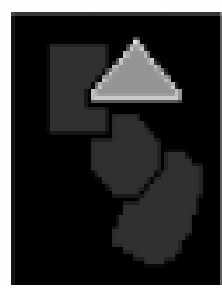

(b)

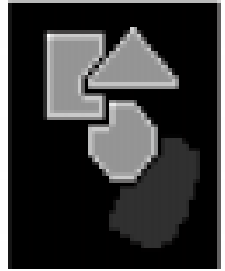

(f)

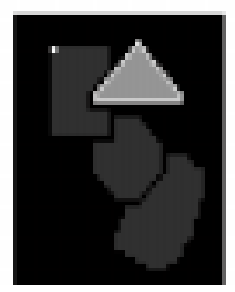

(c)

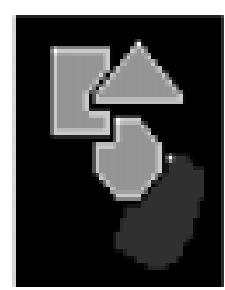

(g)

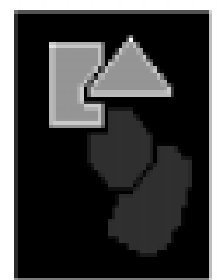

(d)

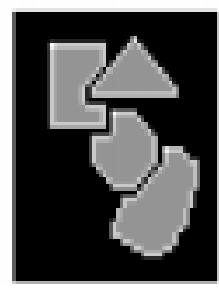

(h)

Figure 4.6 Sequence of Multi-object Detection and Seeding

\subsection{Edge Detection, Region Growing and Particle Projected Area}

The edge following point (EFP) traverses counter-clockwise along the edge of the detected object from the seed. Fig. 4.7 (a) illustrates a simulated object. During the edge traverse, the object interior points are scanned row by row, and counted before the 
EFP moves to the next edge position below, as shown in Fig. 4.7 (b). Logic tests keep the algorithm from double-counting areas or missing parts of irregular objects. The scanning ends at the point where the original seed is reached again, as shown in Fig. 4.7 (c). At the end of the scanning, the particle edge and all of its interior points have been labeled and counted, and each edge point is stored in an ordered list.

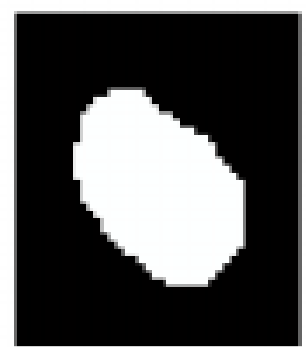

(a) before growing

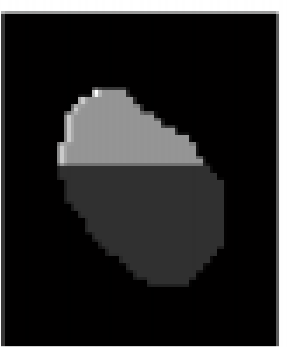

(b) during growing

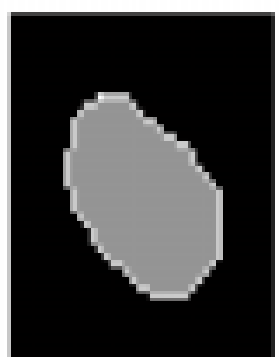

(c) finished growing

Figure 4.7 Region Growing

Fig. 4.8 demonstrates the edge traverse recording. The solid curve shows the vertical moving trajectory of the EFP, while dashed curve shows its horizontal moving trajectory. Note that both curves start at the same point, i.e., seed, and also end at that point. This shows that the full edge following has been completed. 


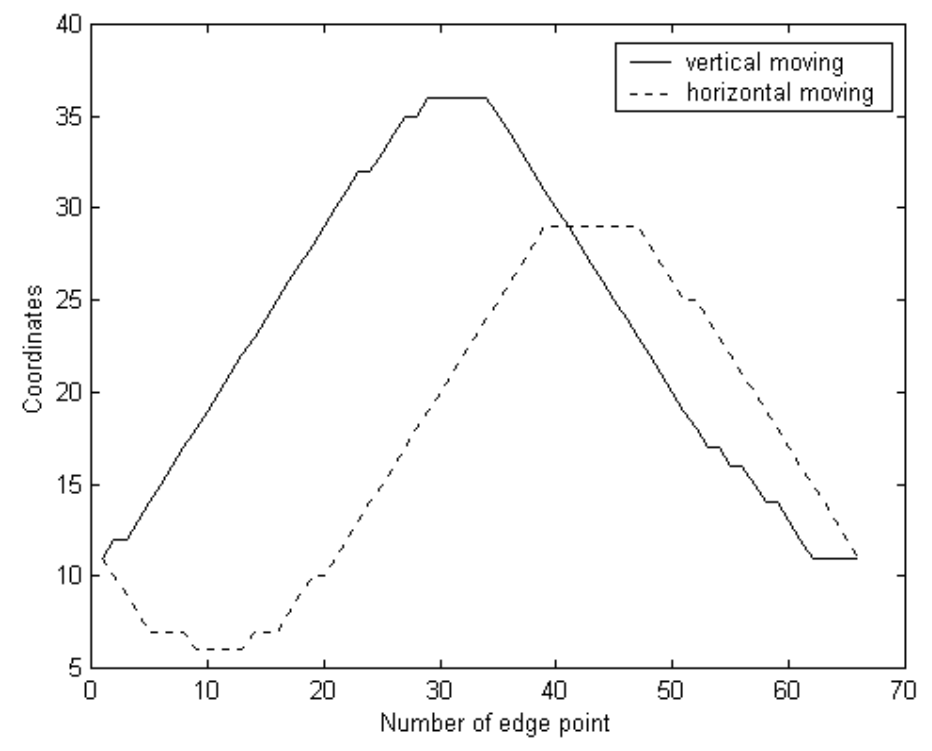

Figure 4.8 Edge Traverse Recording

When the edge following is completed, the total number of pixels on that object is known. Each pixel's area in terms of square millimeters is calculated using the $\mathrm{mm} /$ pixel scale factors and Eqn. (3.1) obtained in the calibration described in Chapter 3. The summation of all the individual pixel areas equals the projected area of that object in question.

\subsection{Centroid Location}

When the region growing process is finished, the total number of edge points of that object is also available. This is accomplished by bookkeeping the number of new points on the edge while traversing is in progress.

In image processing problems, locating the centroid of an object in the 2-D plane is of great importance in shape description and object recognition. In many cases, the 
centroid is used as a reference point to which the position of other points in question can be determined.

For a function $f(x, y)$, the moment of order $(p+q)$ is defined as $m_{p q}$, and the centroid coordinates can be found at

$$
\begin{aligned}
& \bar{x}=\frac{m_{10}}{m_{00}} \\
& \bar{y}=\frac{m_{01}}{m_{00}}
\end{aligned}
$$

where, for a digital image,

$$
\begin{aligned}
& m_{00}=\sum_{x=0}^{m-1} \sum_{y=0}^{n-1} f(x, y) \\
& m_{10}=\sum_{y=0}^{m-1} \sum_{x=0}^{n-1} x f(x, y) \\
& m_{01}=\sum_{x=0}^{m-1} \sum_{y=0}^{n-1} y f(x, y)
\end{aligned}
$$

where $x$ and $y$ indicate the coordinates of the image matrix.

Emdedding Eqn. (4.1) (4.5) in the algrithm, the position of the centroid for each individual shape in the image is located. The centroid finding procedure now is applied to the real image as shown in Fig. 4.9. 


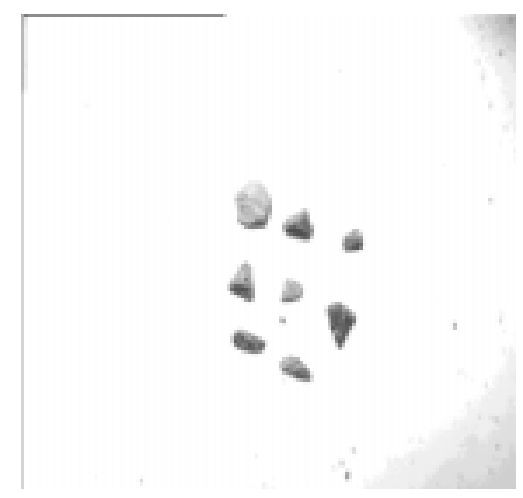

(a)

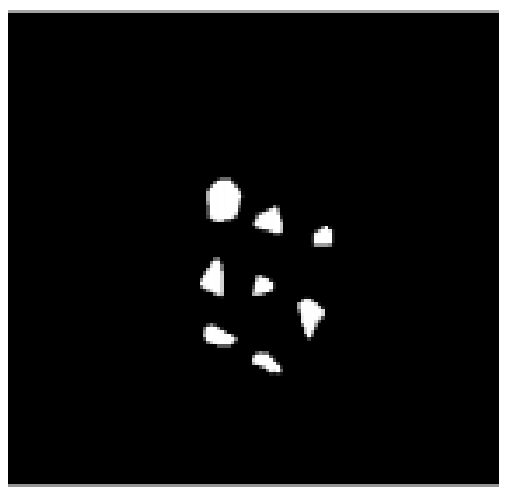

(b)

Figure 4.9 Eight Particles Imaged

Fig. 4.10 shows the centroids found in the eight aggregate particles marked by the cross. Note that in each figure the values on $x$ and $y$ axis are the coordinates which enclose the particle shape in question. In other words, particle's edge is entirely included by the figure border.

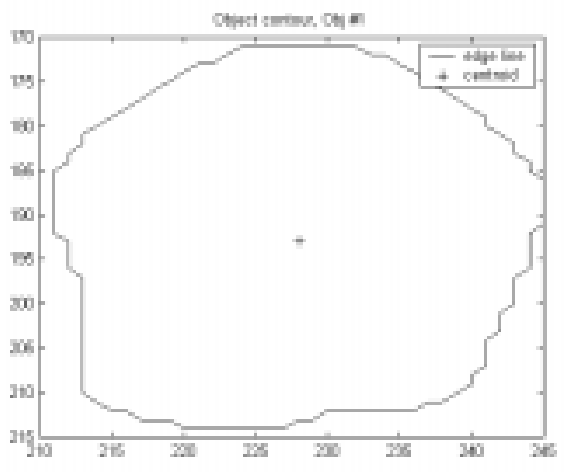

(a) $1^{\text {st }}$ particle

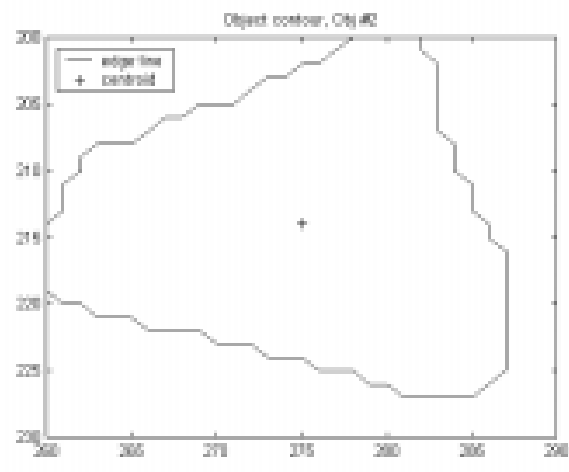

(b) $2^{\text {nd }}$ particle 


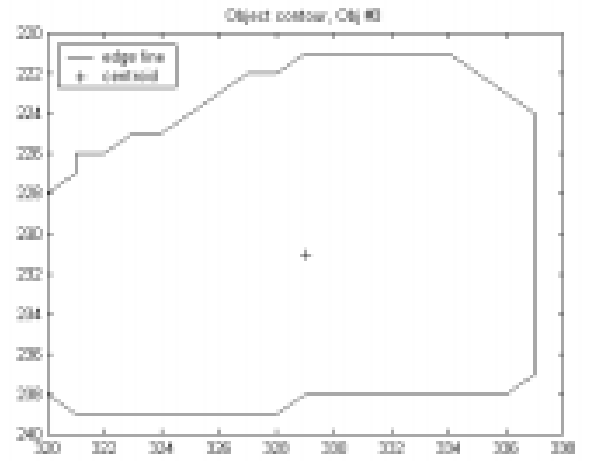

(c) $3^{\text {rd }}$ particle

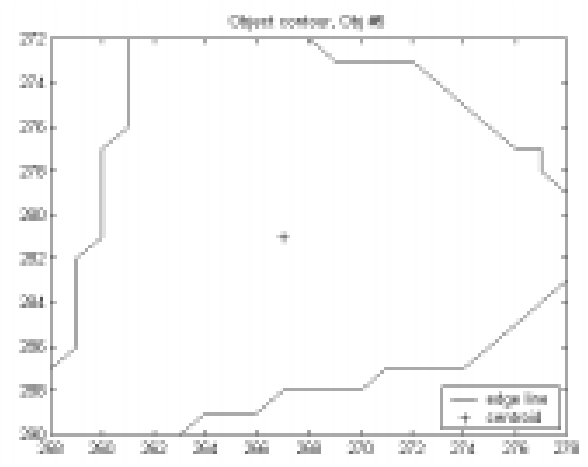

(e) $5^{\text {th }}$ particle

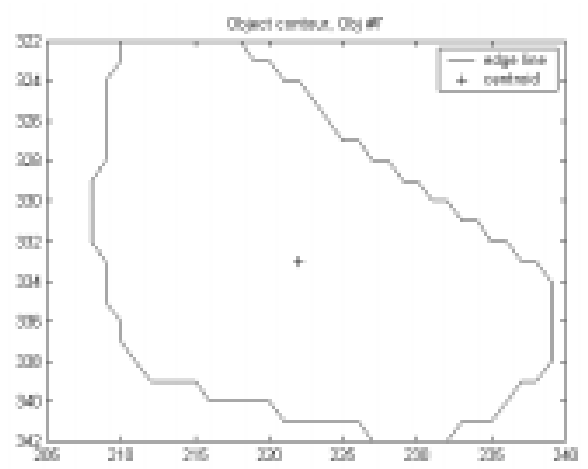

(g) $7^{\text {th }}$ particle

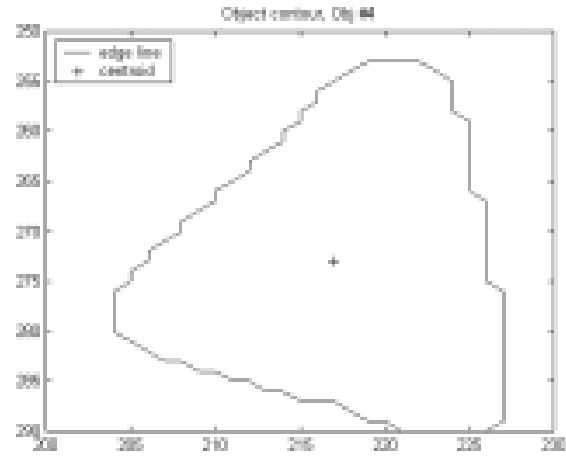

(d) $4^{\text {th }}$ particle

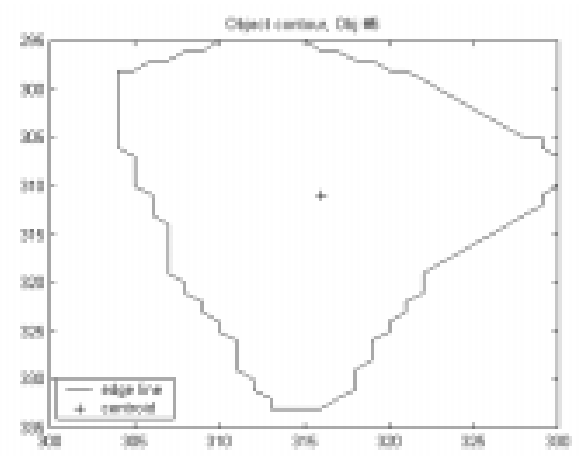

(f) $6^{\text {th }}$ particle

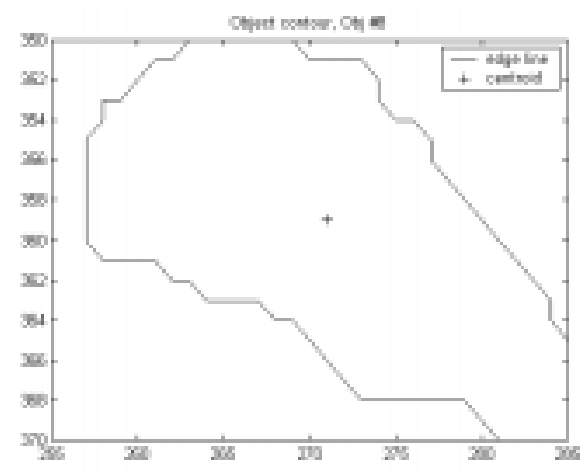

(h) $8^{\text {th }}$ particle

Figure 4.10 Centroid of Profile Shape

Fig. 4.11 (a) shows the binary image of large number of aggregate particles, and (b) shows all the particles that have been processed. 


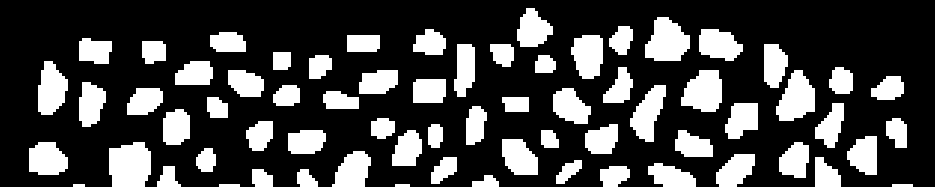

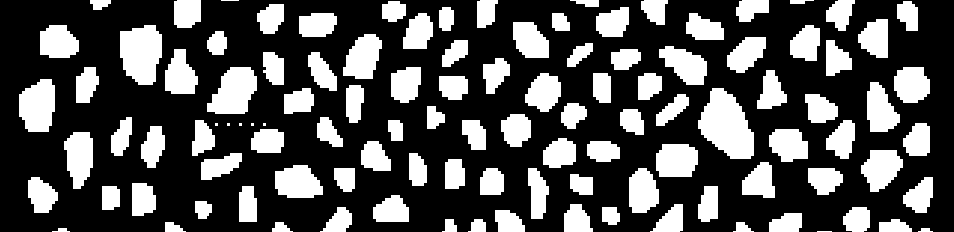

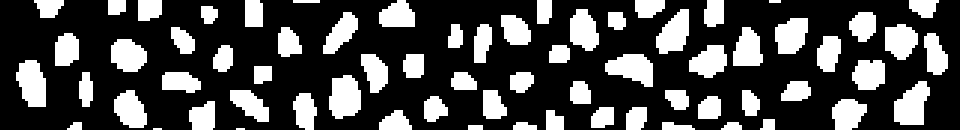

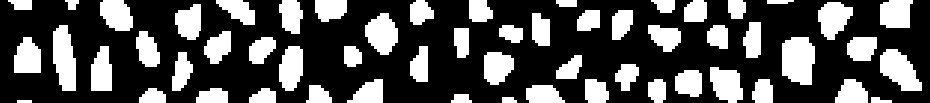

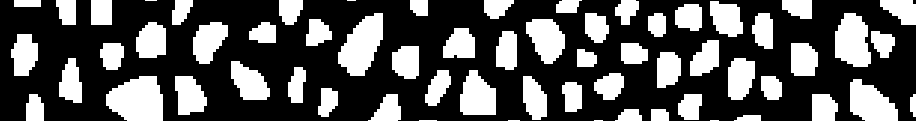

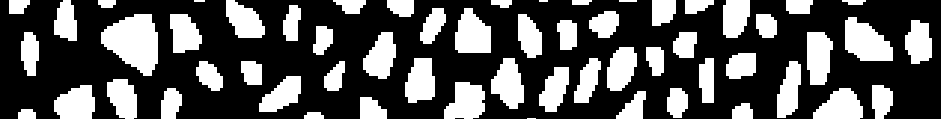

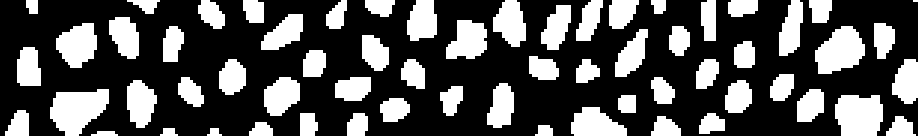

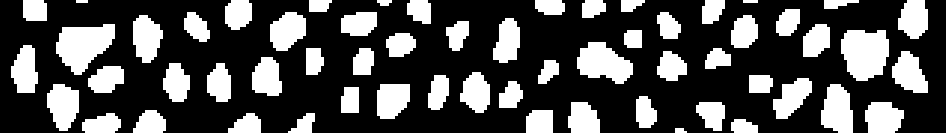

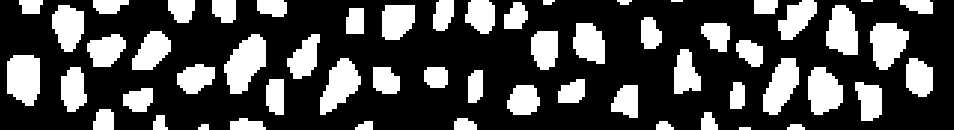

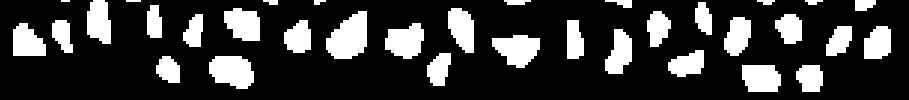

(a) binary image of stock agregates 


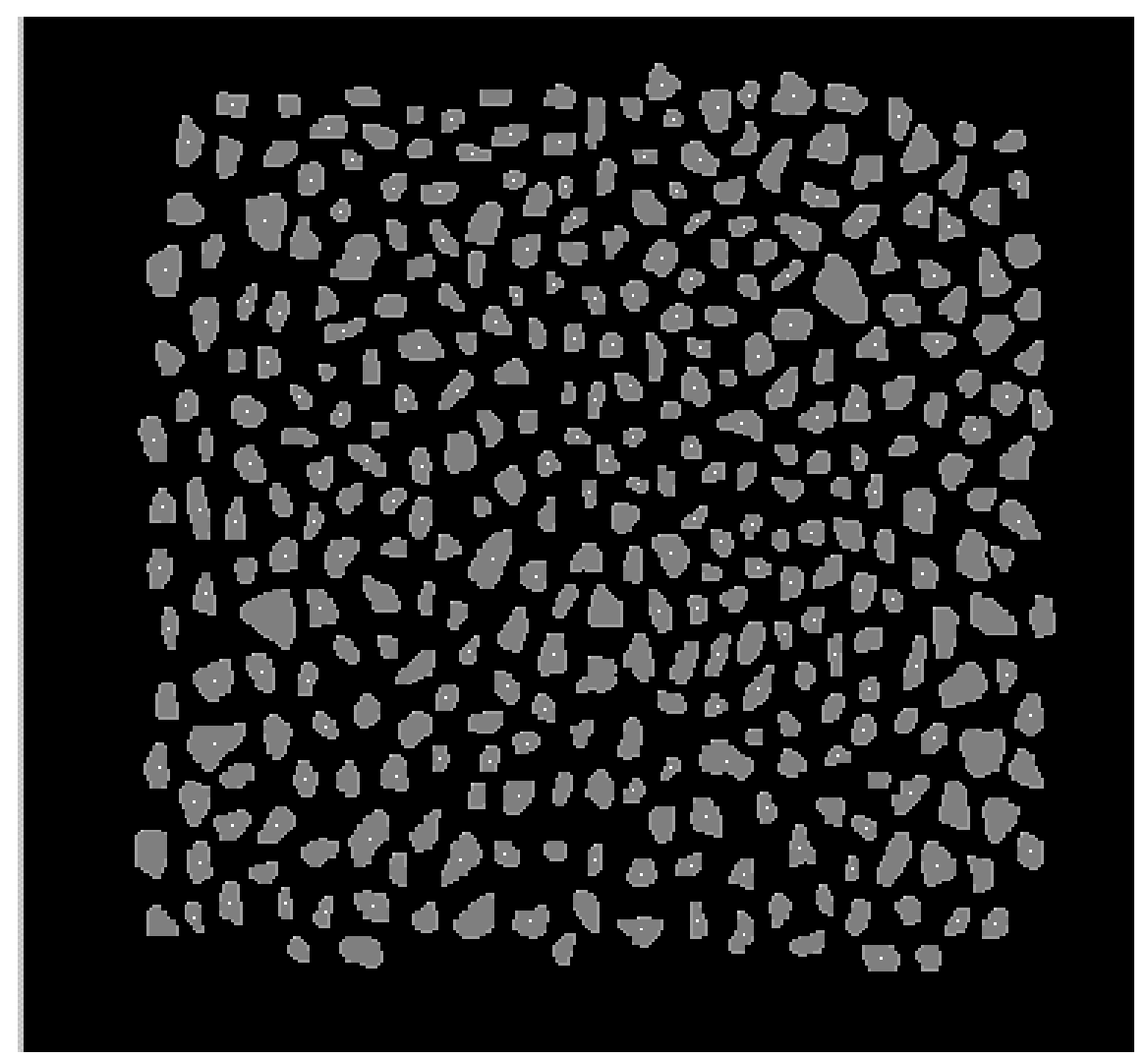

(b) finished particle processing

Figure 4.11 Aggregate Processing

\subsection{Major and Minor Diameter Computation}

Image shape analysis relies on examining a two dimensional silhouette of the object. Techniques for describing the shape measure the simple geometric proportion of the object, such as the perimeter-to-diameter ratio, aspect ratio, etc.. Aspect ratio plays an important role in this research because aspect ratio reflects a particle's elongation, and the length of the minor axis is related to the sieve size. Aspect ratio is defined as the ratio of the maximum diameter to the orthogonal minimum diameter of the shape silhouette. For a random shape, finding its aspect ratio can be performed using principal component analysis, also called "principal eigen analysis". The two end-points of the major diameter 
must be on the major eigen axis, and the two end-points of the orthogonal minor diameter must be on the minor eigen axis, as demonstrated in Fig. 4.12.

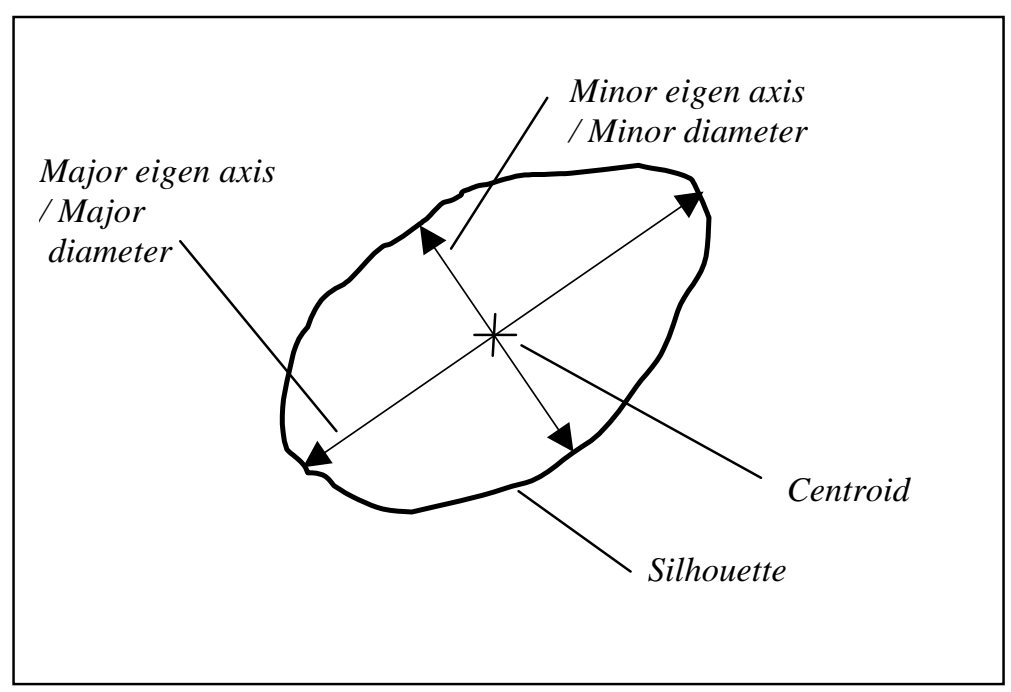

Figure 4.12 Major and Minor Diameter

Consider a set $\mathbf{P}$ of edge point $p(x, y)$ on the contour of interest, with $\mathbf{P}=\left[\mathbf{p}_{1} \mathbf{p}_{2} \ldots\right.$

$\left.\mathbf{p}_{\mathrm{n}}\right]$ and

$$
\mathbf{p}_{i}=\left[\begin{array}{l}
x_{i} \\
y_{i}
\end{array}\right]
$$

The mean vector and the covariance matrix are defined as

$$
\begin{gathered}
\mathbf{m}_{p}=\mathbf{E}\left\{\mathbf{p}_{i}\right\}=\frac{1}{N} \sum_{i=1}^{N} \mathbf{p}_{i} \\
\mathbf{C}_{p}=\mathbf{E}\left\{\left(\mathbf{p}_{\mathrm{i}}-\mathbf{m}_{\mathbf{p}}\right)\left(\mathbf{p}_{\mathrm{i}}-\mathbf{m}_{\mathrm{p}}\right)^{\mathbf{T}}\right\}
\end{gathered}
$$

where $T$ indicates vector transpose, $E$ is the expected value operator. Because $\mathbf{p}_{\mathrm{i}}$ and $\mathbf{m}_{\mathrm{p}}$ are two dimensional, $\mathbf{C}_{\mathrm{p}}$ must be a matrix of order $2 \times 2$. Element $c_{i i}$ of $\mathbf{C}_{\mathrm{p}}$ is the variance 
of $x$ and $y$ in $\mathbf{p}_{\mathrm{i}}$, and element $c_{i j}$ of $\mathbf{C}_{\mathrm{p}}$ is the covariance between $x$ and $y$. The matrix $\mathbf{C}_{\mathrm{p}}$ is real and symmetric.

For $M$ vector samples, namely, $M$ edge points, the mean vector and covariance matrix are computed as

$$
\begin{gathered}
\mathbf{m}_{\mathbf{p}}=\frac{1}{M} \sum_{i=1}^{M} \mathbf{p}_{i} \\
\mathbf{C}_{\mathbf{p}}=\frac{1}{M} \sum_{i=1}^{M}\left(\mathbf{p}_{i} \mathbf{p}_{i}^{T}-\mathbf{m}_{\mathrm{p}} \mathbf{m}_{\mathrm{p}}^{T}\right)
\end{gathered}
$$

Because the matrix $\mathbf{C}_{\mathrm{p}}$ is real and symmetric, finding a set of orthogonal eigenvectors of dimension 2 is always possible [2]. Let $\lambda_{1}$ and $\lambda_{2}$ be the eigenvalues of $\mathbf{C}_{\mathrm{p}}$, with $\lambda_{1}>\lambda_{2}$, and correspondingly, let $\mathbf{e}_{1}$ and $\mathbf{e}_{2}$ be the resultant eigenvectors. The direction of vector $\mathbf{e}_{1}$ indicates the orientation of the particle's major eigen axis, and likewise, the direction of $\mathbf{e}_{2}$ coincides with the direction of its minor eigen axis. The end-points of the major and minor axes within the contour can be found, so that the major diameter and its orthogonal minor diameter can thus be obtained.

Fig. 4.13 shows the two end-points of major and minor axis found on the edge line of the image shown previously in Fig. 4.9. Note that some major and minor diameters do not appear orthogonal because of pixel's aspect ratio. 


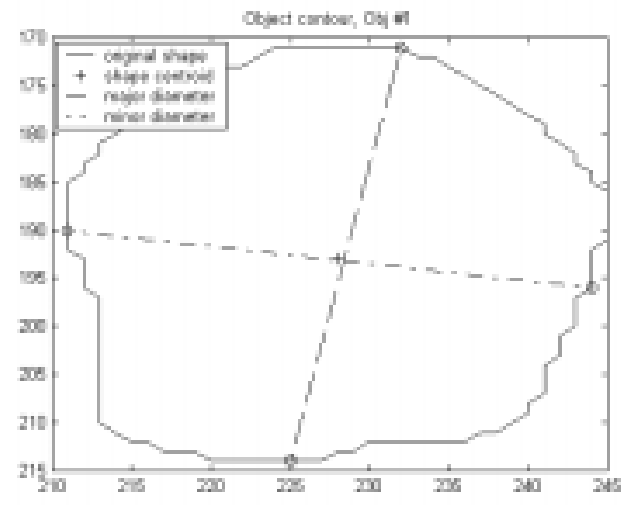

(a) diameters for object \#1

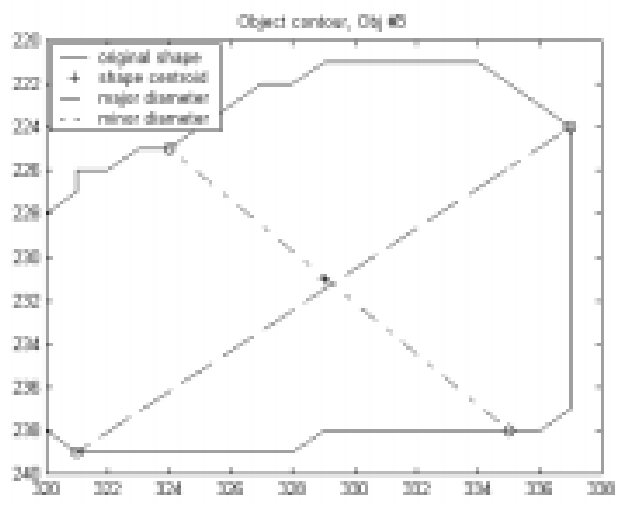

(c) diameters for object \#3

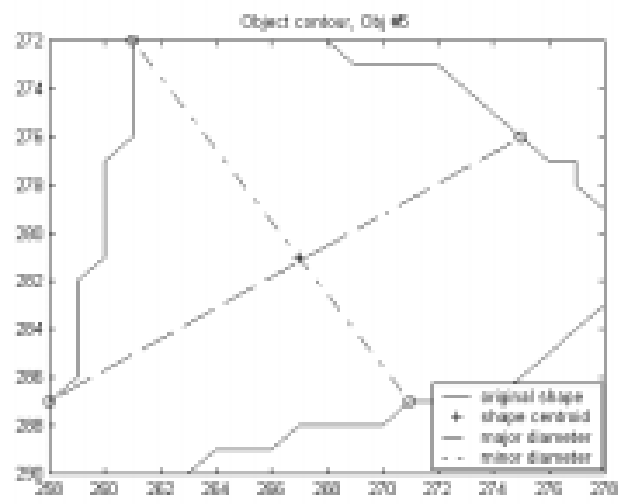

(e) diameters for object \#5

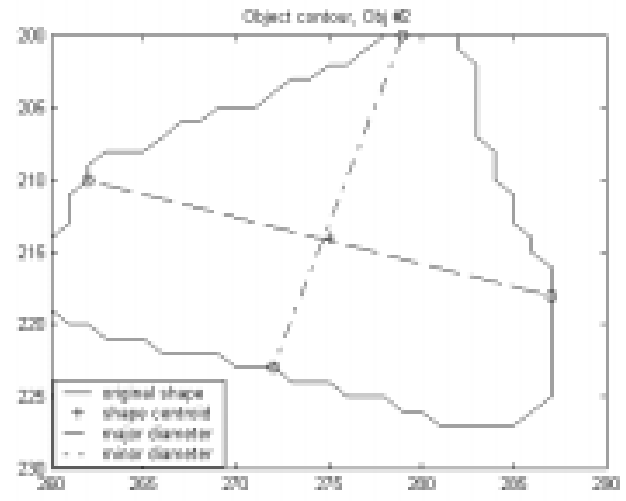

(b) diameters for object \#2

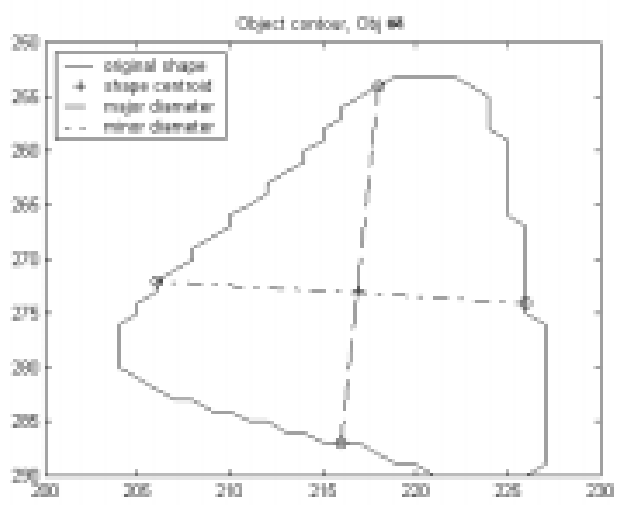

(d) diameters for object \#4

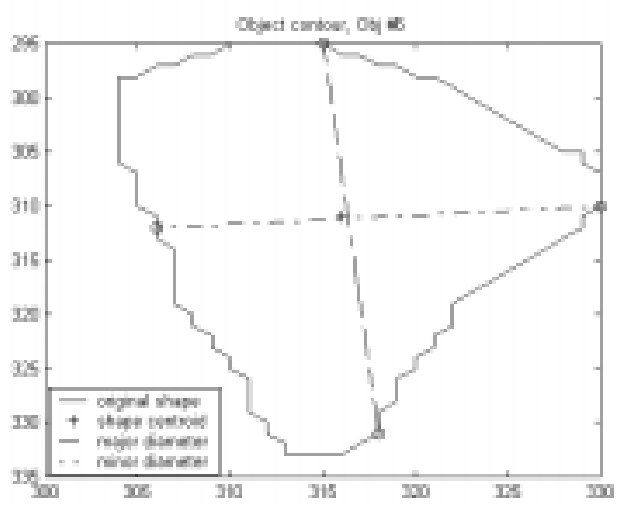

(f) diameters for object \#6 


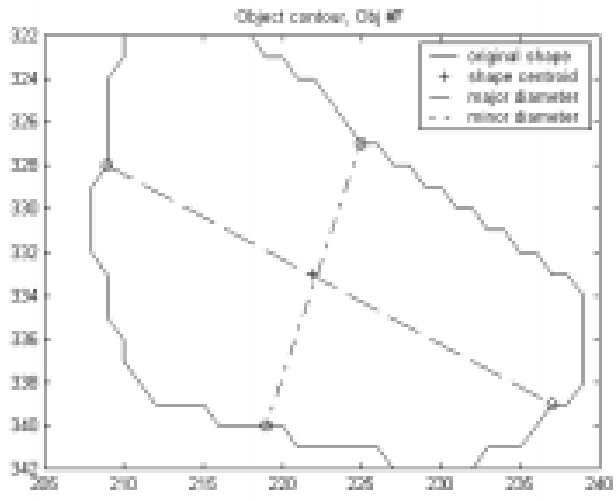

(g) diameters for object \#7

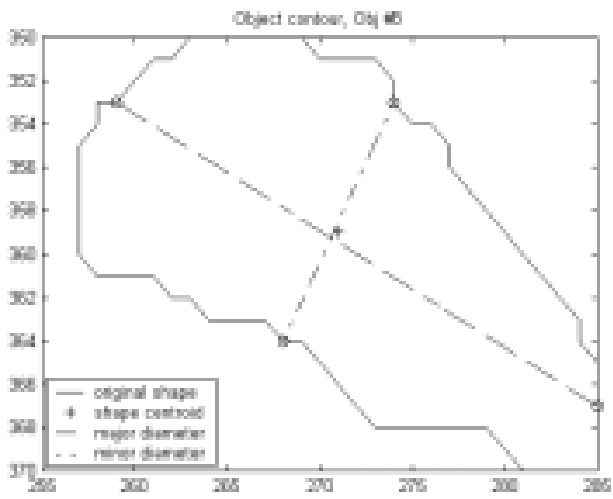

(h) diameters for object \#8

\section{Figure 4.13 Major and Minor Diameter}

After obtaining the major and minor diameter, the aspect ratio of the profile shape of the particle is computed.

\subsection{Profile Shape Characterization}

The major reason for needing to know the approximate shape of the particles lies in the fact that shape affects the strategy for converting the particle profile into an equivalent sieve size. For example, rectangular particles will sieve to the smaller of the two dimensions, which can be found approximately using the minor diameter. On the other hand, a triangular shaped particle will sieve to one vertex and the opposite side, the length that is sieved to is greater than the minor diameter of the profile shape. This requires modification of the minor diameter.

Using the list of edge points to plot the radius from the centroid to each edge point, a relation called "signature" is constructed. Fig. 4.14 illustrates such a functional relation for a square. Irrespective of how such a signature is created, the basic idea 
remains to reduce the boundary description to a 1-D function, which is easier to describe than the original 2-D contour.

Signatures generated in this way are invariant to translation, but they do depend on rotation and scaling. To achieve invariance of the signature to both scaling and orientation, the plot may be normalized by finding a consistent way to select the same starting point to generate the signature. For instance, the edge point, which has the maximum radius to the centroid, can be selected to start calculating the radius. The maximum radius is also used to scale all signatures to a uniform range, $[0,1]$. The normalization step removes dependency on size and rotation but preserves the fundamental shape of the particle's contour.

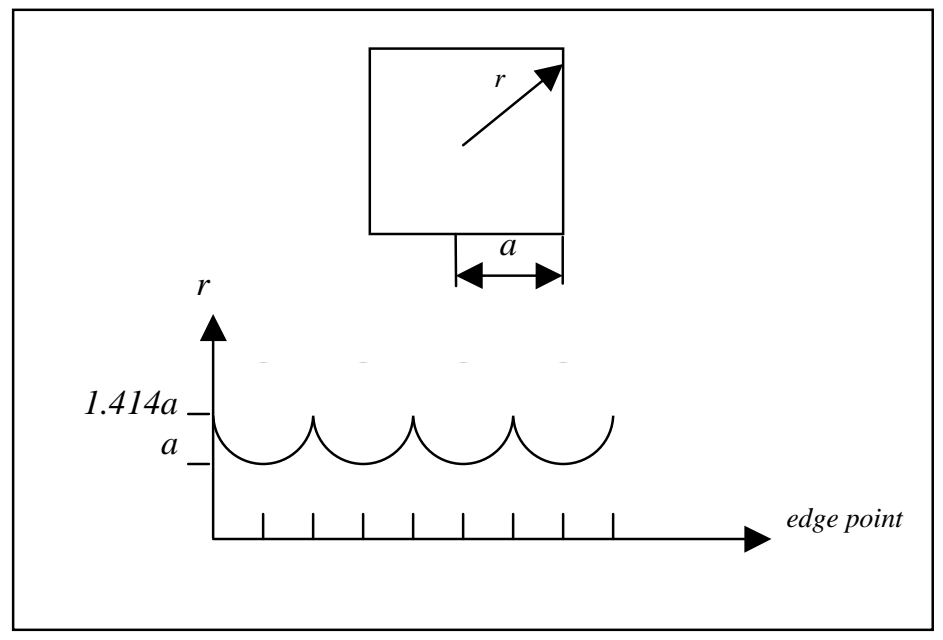

Figure 4.14 Signature

Signatures are used to store and reveal the profile shape information. Analyzing their patterns can give certain shape characteristics. For example, a relatively straight 
line parallel to the abscissa indicates that the object's shape is circular. If the signature of a convex shape has four sharp peaks of the same radius and four smooth valleys also of the same radius, and all are evenly spaced along the abscissa, it can be deduced that this object's shape is square shaped. Three peaks and three valleys in a signature imply a triangular shape in a general sense. For a convex object, its shape can be characterized by its simplified contour obtained from connecting all the characteristic vertices, provided these characteristic vertices can be detected. Fig. 4.15 demonstrates this idea, in which the contour $\overline{A B C D}$ can be used to characterize the object's original shape.

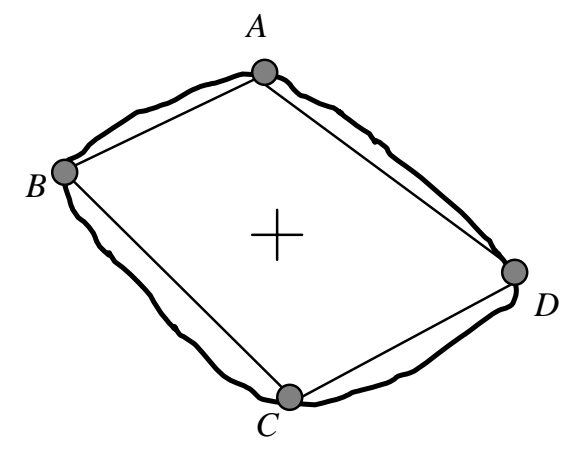

\section{Figure 4.15 Characterizing a Shape}

Although all the vertices can be extracted from the signature by observing its first derivative quantities, identifying a few characteristic ones is not an easy task. This is because the signature of an imaged object usually is very noisy, and many points that may not serve the characterizing purpose may be picked as major vertices, making the shape feature description complex. In order to efficiently find the characteristic vertices, noise must be removed. 
Polynomial curve-fitting can effectively approximate functions (interpolating polynomials) to smooth out noisy experimental and numerical data, and provide a simple analytical expression. The most commonly chosen form is the polynomial:

$$
g(x)=a_{0} x^{p}+a_{1} x^{p-1}+a_{2} x^{p-2}+a_{3} x^{p-3}+\ldots+a_{p-2} x^{2}+a_{p-1} x+a_{p}
$$

where $x$ is the variable of edge points.

Determination of the order of the polynomial $p$ is problem dependent. For a given set of data points, an order too high causes detection of unwanted and insignificant vertices, an order too low lacks sensitivity of detection. After trial-and-error, $p=18$ was selected in this research. After the order was chosen, the first derivative of the polynomial was taken to identify characteristic vertices, using:

$$
\frac{d g(x)}{d x}=0
$$

to locate the positions of the desired vertices on the original signature. The number of maxima and minima is an indicator to the number of "corners" and "sides" that the particle has.

Now, taking the same images as shown in Fig. 4.9, the selected polynomial is applied to identify these eight particles' profile shape. Fig. 4.16 demonstrates the results. All plots in the left column show the polynomial curve-fitting effect, and shapes identified on the other. 


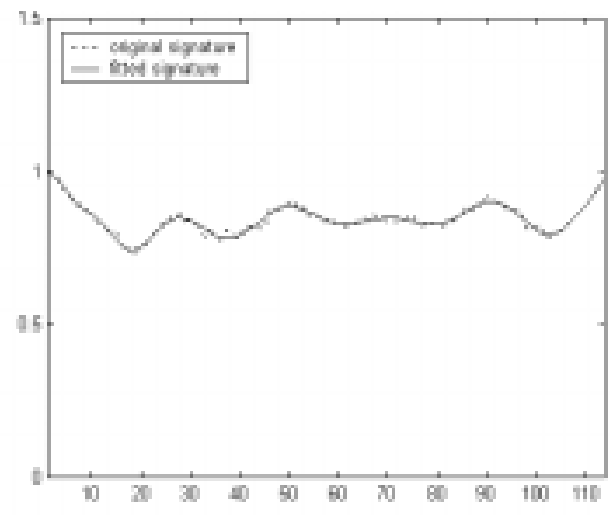

(a) curve-fitting for object \#1

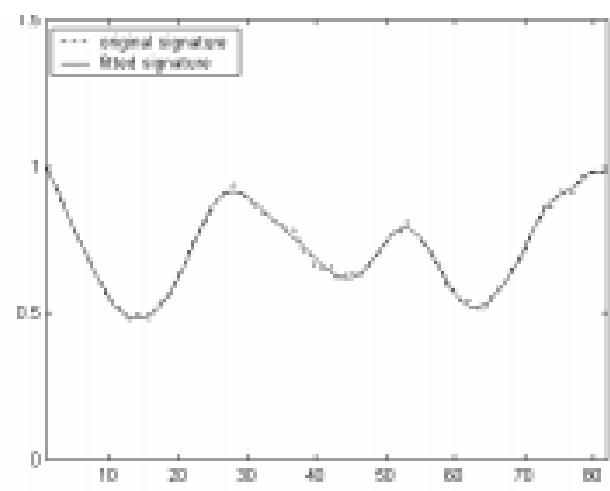

(c) curve-fitting for object \#2

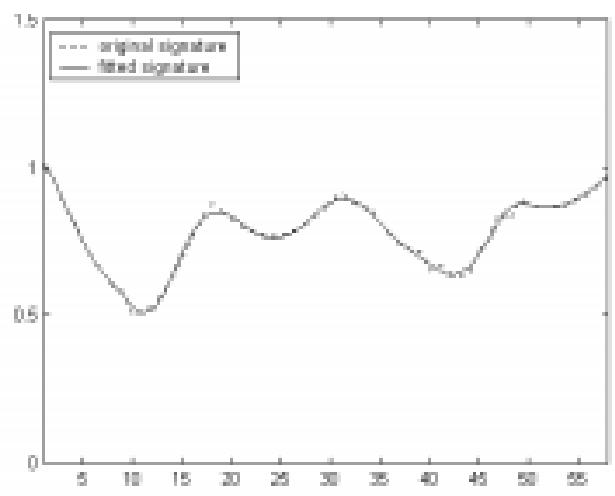

(e) curve-fitting for object \#3

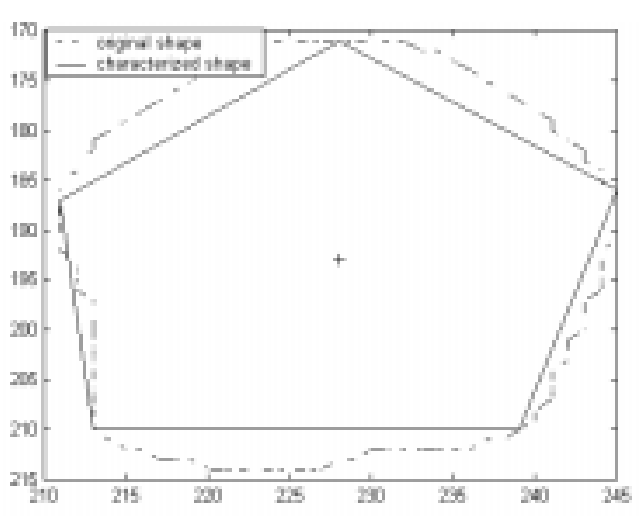

(b) characterized shape for object \#1

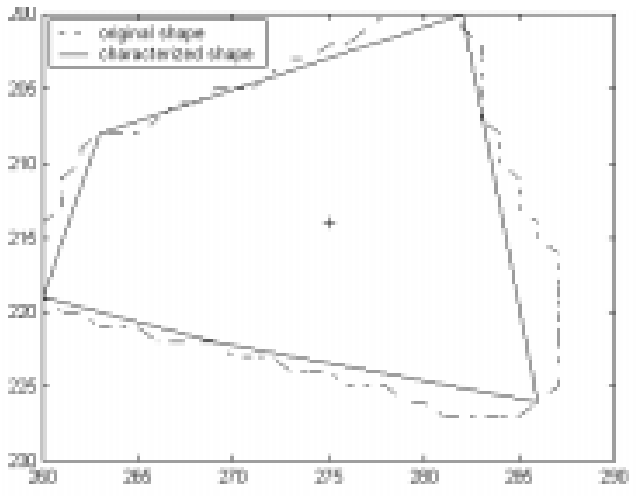

(d) characterized shape for object \#2

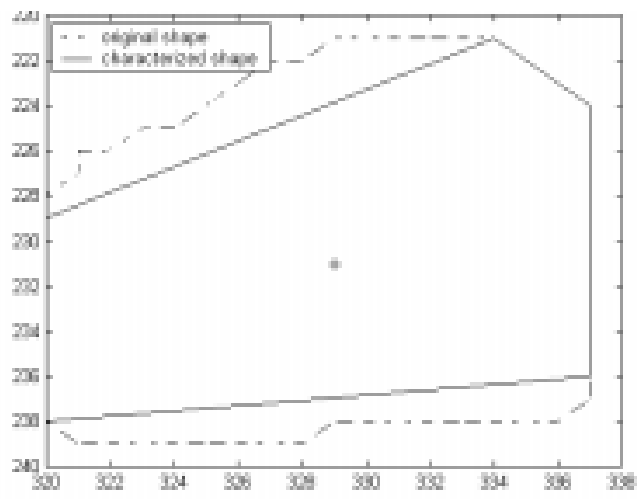

(f) characterized shape for object \#3 


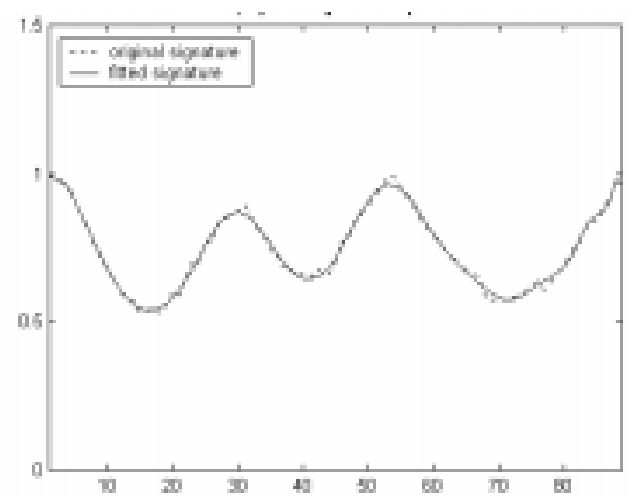

(g) curve-fitting for object \#4

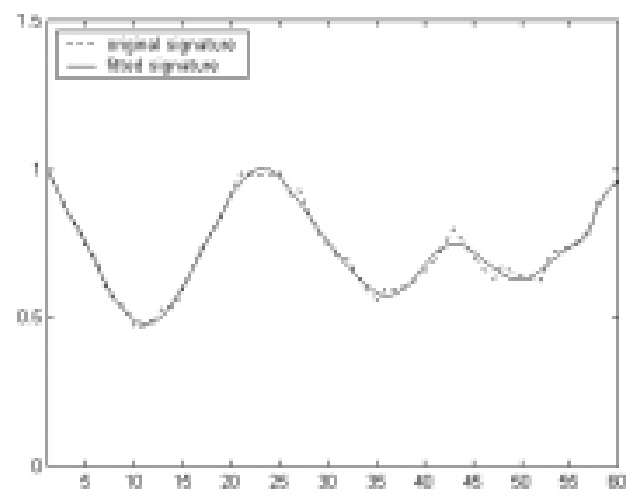

(i) curve-fitting for object \#5

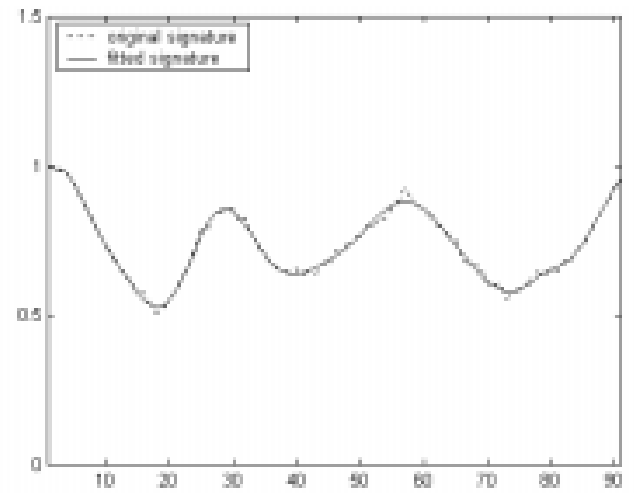

(k) curve-fitting for object \#6

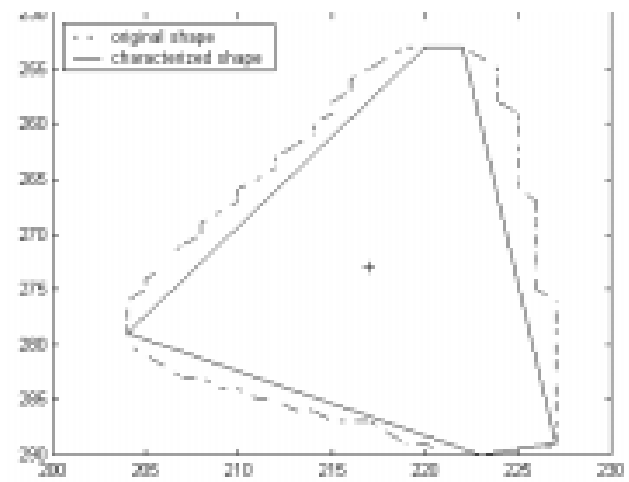

(h) characterized shape for object \#4

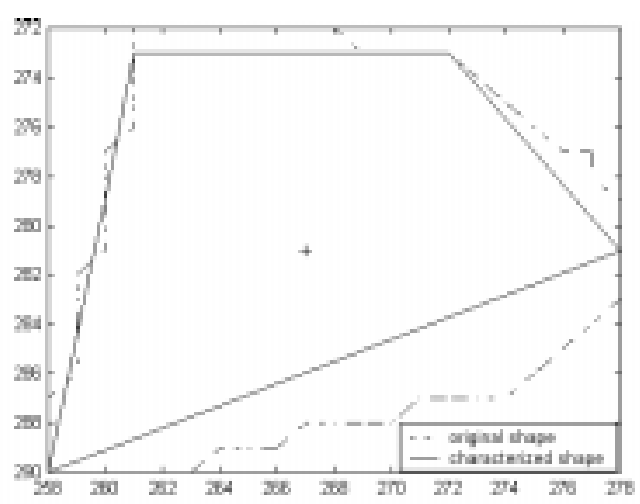

(j) characterized shape for object \#5

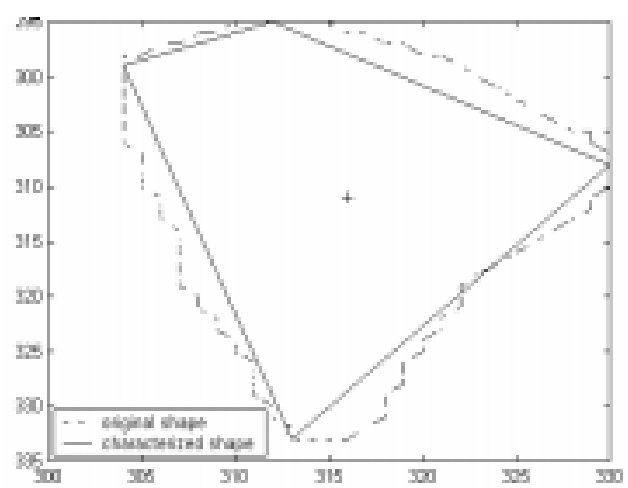

(1) characterized shape for object \#6 


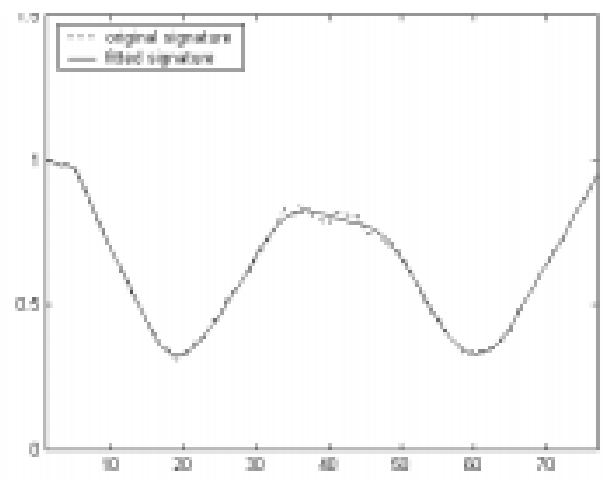

(m) curve-fitting for object \#7

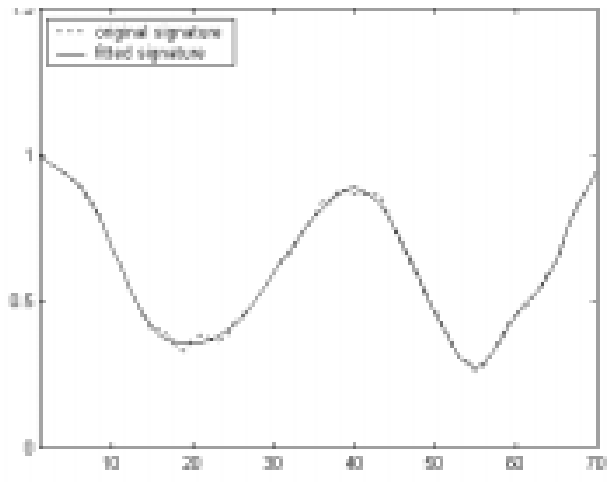

(o) curve-fitting for object \#8

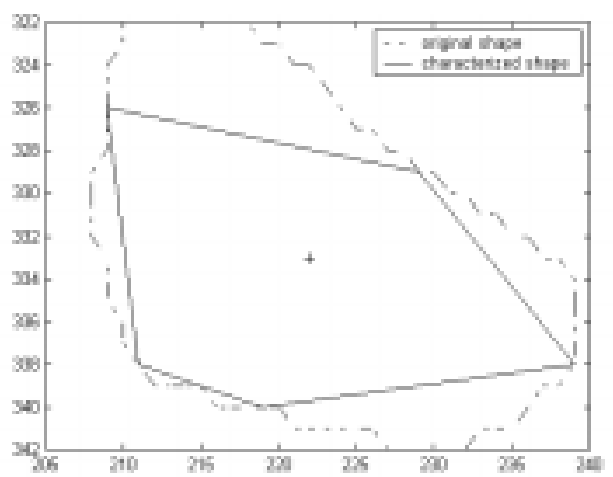

(n) characterized shape for object \#7

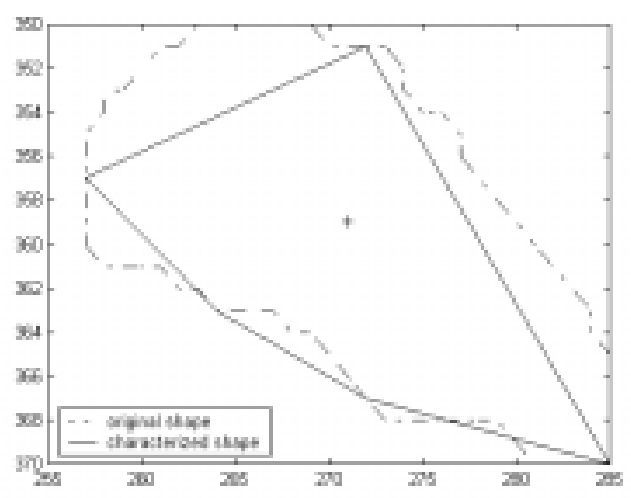

(p) characterized shape for object \#8

\section{Figure 4.16 Curve-fitting and Shape Characterization}

Note that some obviously triangular shapes are "over characterized", which means that an expected triangular shape is identified as four or five-sided shape instead. Naturally, reducing the order of the polynomial may be able to partially overcome this drawback. However, the side effect is inevitable, that is, some true four or five-sided shapes may be mistaken as triangles. So, in the algorithm of this research, it is regulated that if the number of edge points covering a side length of the shape is less than $1 / 8$ the total number of edge points, this side can be ignored. 


\section{SEPARATION OF TOUCHING AND OVERLAPPING PARTICLES}

\subsection{Introduction}

In the processing of aggregate particle images, two problems must be solved before size and shape analysis begin. First, if the particles are touching or overlapping, two or more particles will appear as one large, irregularly shaped particle. Second, each image consists of many individual particles, all of which must be processed individually to determine particle size, shape and mass. These two problems demand separation of all touching or overlapping particles before further analysis can be conducted on the image.

Morphological processing techniques can be used to convert the binary image to a gray scale topographic surface [21]. In this chapter, some basic morphological concepts are reviewed. The 3-D geometric characteristics existing between two touching or overlapping objects are analyzed. A morphological erosion process is demonstrated, which leads to finding a saddle point in a concave particle outline. A cut line is made through the saddle point and eventually the two objects are separated.

\subsection{Binary Erosion}

The fundamental operations of mathematical morphology are erosion and dilation. In this work, erosion is the more important process, and can be described as follows: suppose a binary image $\mathbf{I}_{\mathrm{m} \times \mathrm{n}}$ contains background pixels with value 0 and object pixels with value 1 . Assume that the object pixels are grouped into a single, contiguous object A comprised of $q$ pixels $a_{1}, a_{2}, \ldots a_{q}, q<\mathrm{m} \times \mathrm{n}$. Let $\mathbf{B}=\left\{b_{1} b_{2} \ldots b_{k}\right\}$ be a structuring element, which is a set of binary points that are usually (but not necessarily) contiguous 
and that usually (but not necessarily) describes some geometric shape - a line, disk, rectangle, etc. Let $b_{r}$ be the reference point for B. Note that $b_{r}$ is usually (but not necessarily) an element of $\mathbf{B}$. The structuring element $\mathbf{B}$ moves over the image. When $\mathbf{B}$ is completely contained in $\mathbf{A}$, the reference point location goes into the eroded set. In other words, an eroded image is constructed by a set that is made up of the locations of the reference point of structuring element $\mathbf{B}$, for those locations $\mathbf{B} \subset \mathbf{A}$. Fig. 5.1 portrays a typical erosion process.
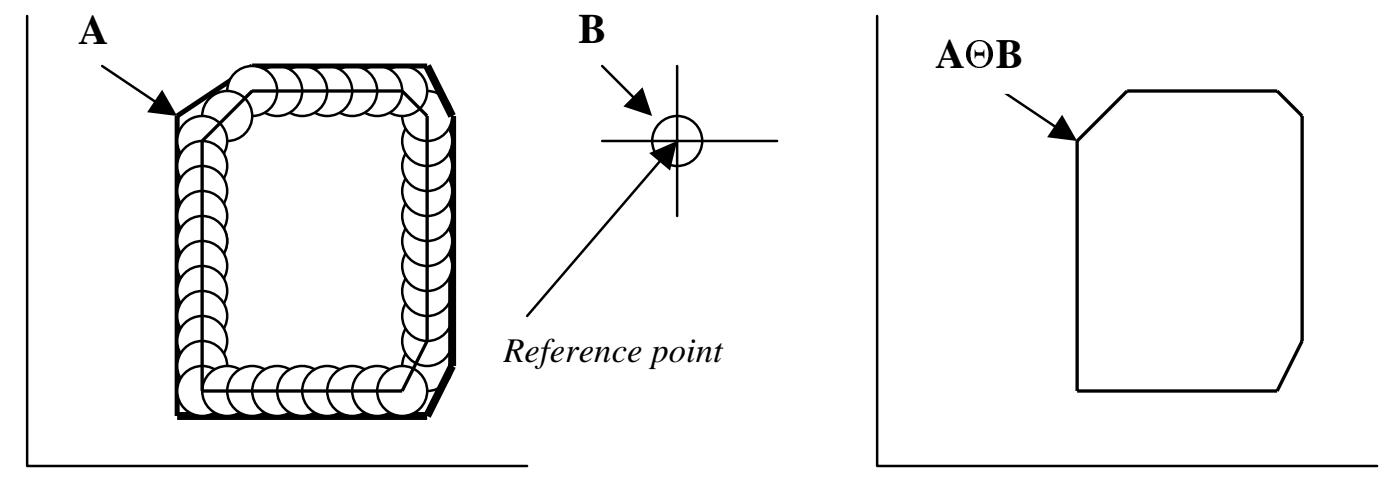

Figure 5.1 Typical Erosion as Shrinking

\subsection{Sequential Erosion}

The erosion process is performed by mathematically "moving" the structuring element over the object image. At each location, a simple Boolean process is used. If the structuring element is completely contained in the object to be eroded, the pixel location corresponding to the reference point of the structuring element is placed in the new set, which forms the eroded object. Otherwise, the reference pixel becomes background in the new image that contains the eroded object(s). 
In a sequential erosion, when the eroded object can not be eroded any more, in other words, at the point when the eroded object can not entirely contain the structuring element any more, this state is called ultimate erosion.

Sequential erosion on binary images is widely used in morphological processing. For example, computation of gradients, marking functions, distance functions are based on sequential erosion [21]. The basic mechanism is that as an erosion is performed, the eroded section is labeled with a value that increases with each erosion operation. The original binary image is thus converted to a gray-scaled 'mountain'. The brightest part (largest gray value, or highest altitude) is located at the ultimate erosion for that shape. Fig. 5.2 illustrates the sequential erosion process. 


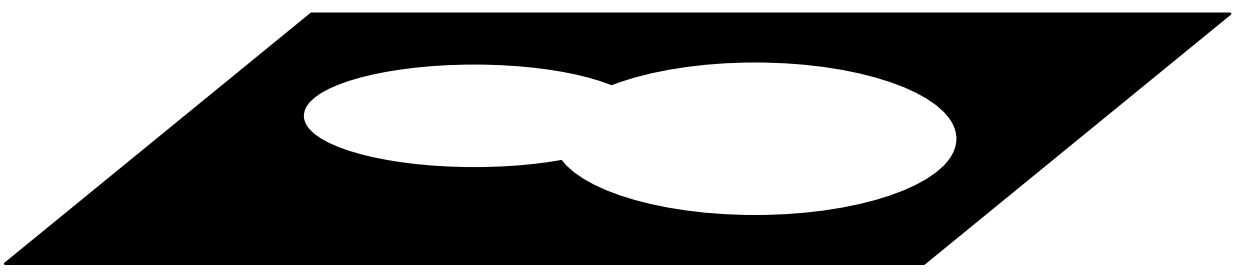

(a) original binary image

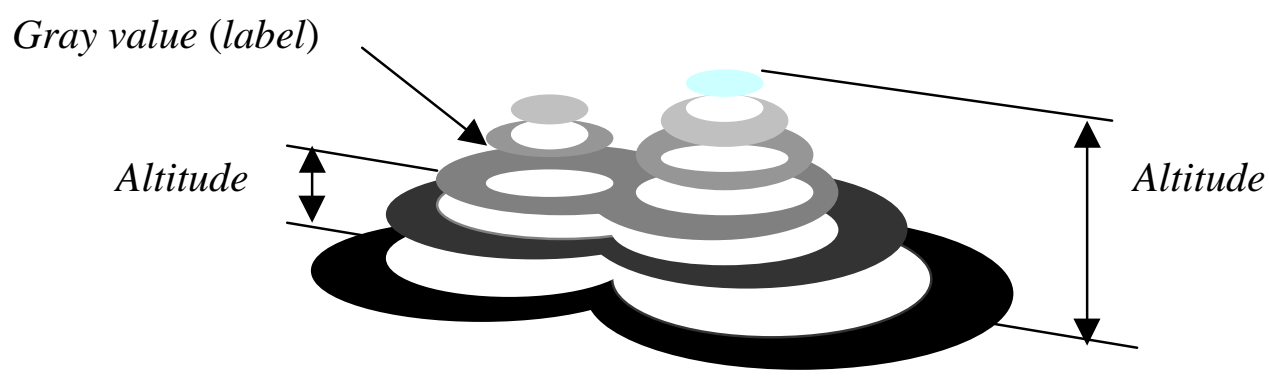

(b) after ultimate erosion (oblique view)

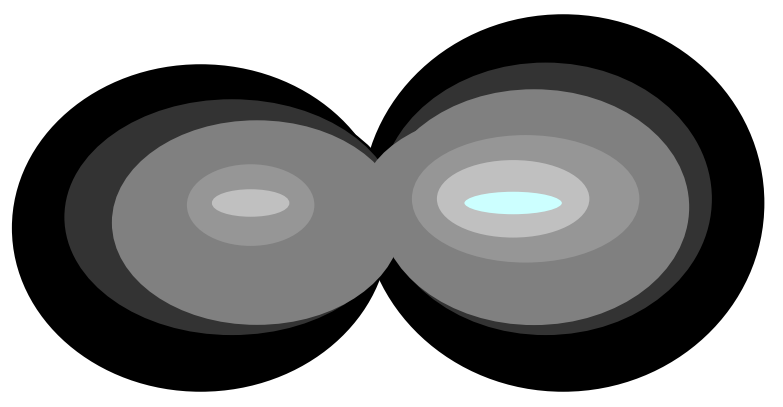

(c) after ultimate erosion (top view: topographic surface)

\section{Figure 5.2 Sequential Erosion}

Fig. 5.3 shows the whole sequential erosion process for a simulated image on a matrix of size $44 \times 40$ in which touching and overlapping take place. A structuring element of size $3 \times 3$ was used. 


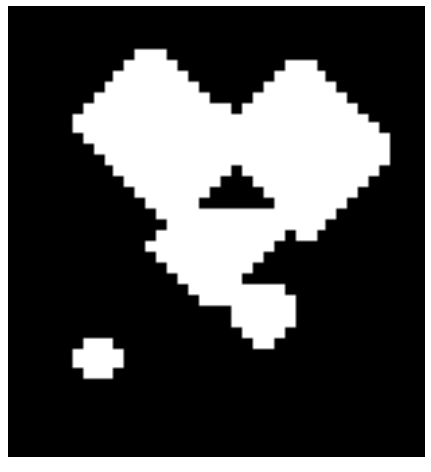

(a) before erosion

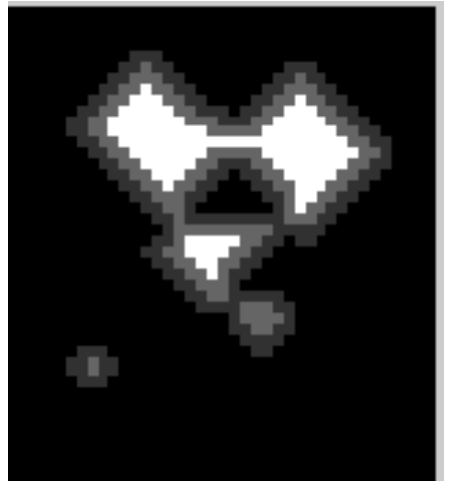

(c) $2^{\text {nd }}$ erosion

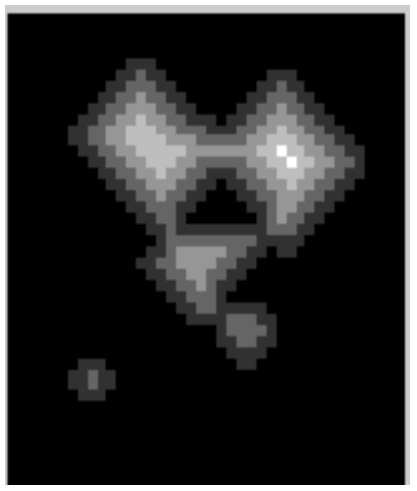

(e) $4^{\text {th }}$ erosion

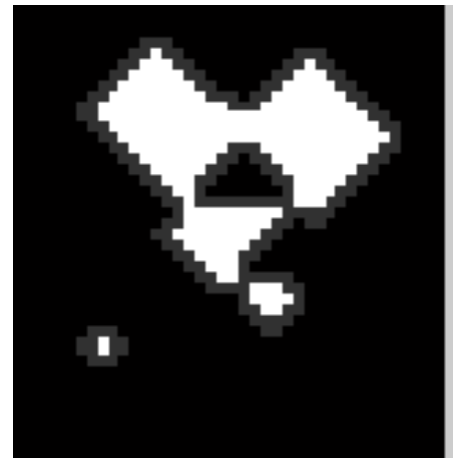

(b) $1^{\text {st }}$ erosion

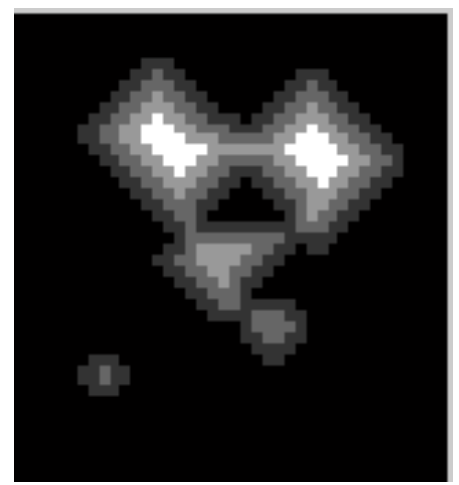

(d) $3^{\text {rd }}$ erosion

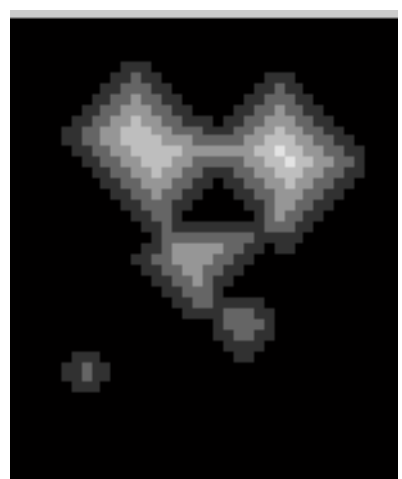

(f) ultimate erosion

Figure 5.3 Sequential Erosion on Simulated Image 
The pseudocode of the sequential erosion is listed in Table 5.1 below.

\section{Table 5.1 Pseudocode of Sequential Erosion}

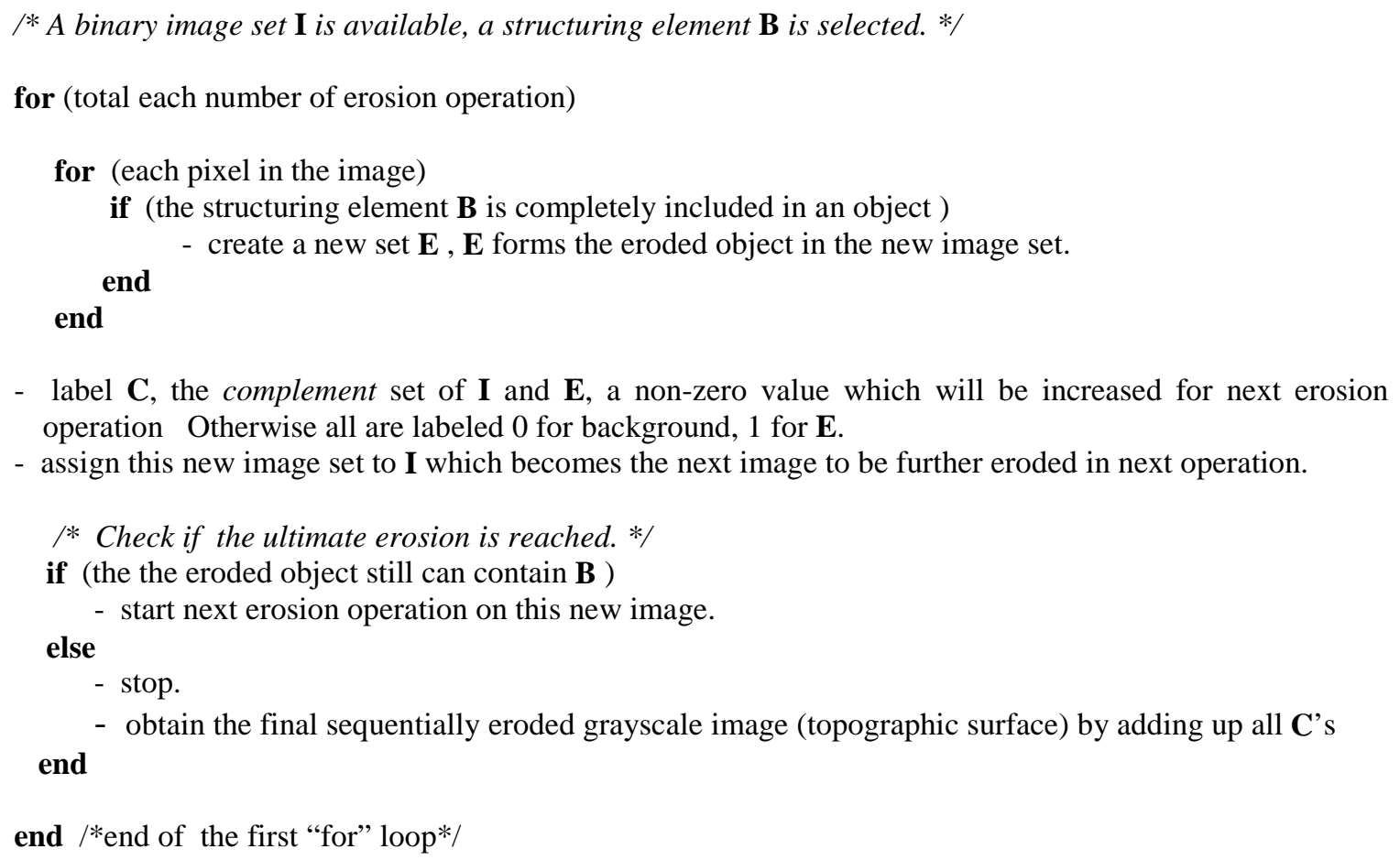

- label $\mathbf{C}$, the complement set of $\mathbf{I}$ and $\mathbf{E}$, a non-zero value which will be increased for next erosion operation Otherwise all are labeled 0 for background, 1 for $\mathbf{E}$.

- assign this new image set to I which becomes the next image to be further eroded in next operation.

/* Check if the ultimate erosion is reached. */

if (the the eroded object still can contain $\mathbf{B}$ )

else

- start next erosion operation on this new image.

- stop.

- obtain the final sequentially eroded grayscale image (topographic surface) by adding up all C's end

end $/ *$ end of the first "for" loop*I

The total number of erosion operations necessary to reach the ultimate erosion and morphological gradient of the eroded grayscale image depends on structuring element size, and on the size and shape of the object. The smaller the structuring element size (greater than one pixel, of course), the more topographic information can be revealed. Obviously, this comes with a trade-off of longer run time for computer. 


\subsection{Separating Location}

As shown in Fig. 5.3, successive bands (or rings) created by the erosion are assigned grayscale values, increasing with each erosion operation. The bands generate a contour for each object, similar to a topographic contour map. After the ultimate erosion, a complete topographic surface is formed. An inevitable characteristic for those touching and overlapping object shapes can be noted: a saddle shaped surface exists between two connected objects in the topographic plane. The erosion process serves the purpose of creating the topographic surface, on which the right place to cut these two connected objects apart is located. The right place to cut is located at the "saddle point", which mathematically is the point where a 3-D function simultaneously reaches a (local) minimum in one direction and a (local) maximum in the other direction. Examples would be the center of a saddle seat or the lowest point (gap) in a ridge between two hills. Fig. 5.4 depicts the saddle shaped surface over two connected hills and saddle point location. Note that the saddle point $S$ has the minimum value in the plane $A$, and meanwhile the maximum value in the plane $B$. Both planes are perpendicular to the background and intersect each other through the saddle point. 


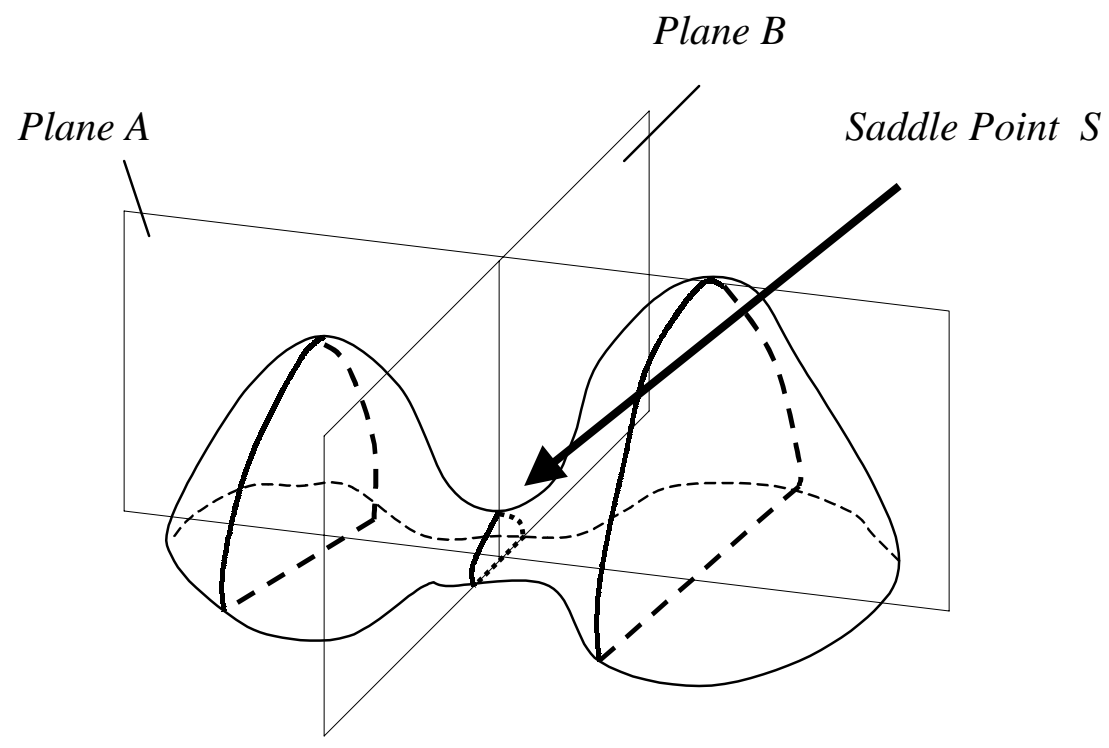

Figure 5.4 Saddle and Saddle Point

The saddle point in the ultimately eroded image shown in Fig. 5.3 (f) can be located. Fig. 5.5 (a) shows the two dissecting plane locations to find the saddle point between two hills, and (b) illustrates these two corresponding plane locations in the topographic map. Mathematically, the point set in each plane constructs a parabolic curve, with opposite opening direction. The saddle point is located at the intersecting point of these two curves. Note that the same saddle point has the minimum value for the set of all the gray value points in the plane $A$, and the maximum value in the plane $B$. Notationally, let $\mathbf{A}$ be the set of all gray value points in the plane $A$, and $\mathbf{B}$ the set of all gray value points in the plane $B, s$ the gray value at the saddle point, then the following equations hold:

$$
\begin{aligned}
& s=\min \left\{a_{i}, \forall a_{i} \in \mathbf{A}, i=1,2, \ldots, n\right\} \\
& s=\max \left\{b_{i}, \forall b_{i} \in \mathbf{B}, i=1,2, \ldots, m\right\}
\end{aligned}
$$


Once the saddle point is located, next is to determine the direction of cutting. The cutting line is contained in the plane that contains maximum gray value with the parabolic curve opening downward. In the above example, the cutting line is contained in plane $B$.

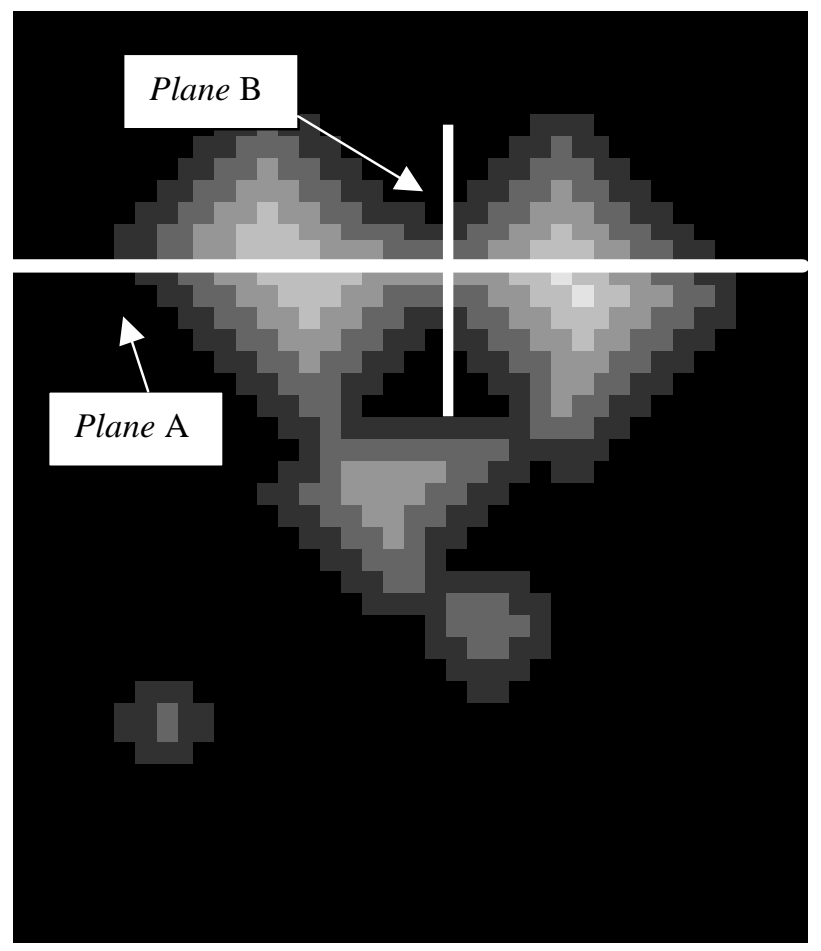

(a) dissecting planes (top view) 


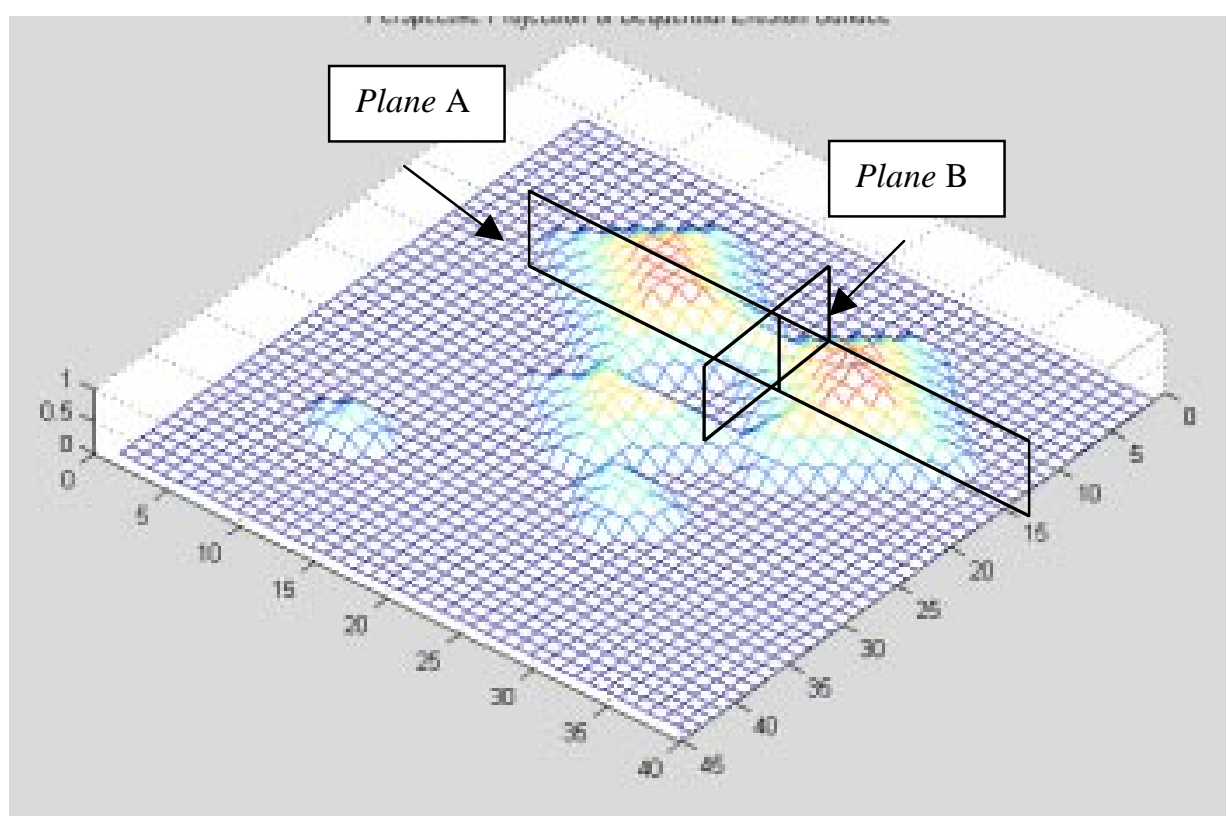

(b) dissecting planes (oblique view)

Figure 5.5 Dissecting Planes for Finding Saddle Point

More specifically, Fig. 5.6 (a) shows the altitude in gray value in dissecting plane $A$ with a fitting parabola opening upward. Similarly, (b) shows the altitude in dissecting plane $B$ with a fitting parabola opening downward. Note that both sets intersect at gray level of 0.6 , and the desired saddle point is located at position $(13,22)$ with the gray level 0.6 . 


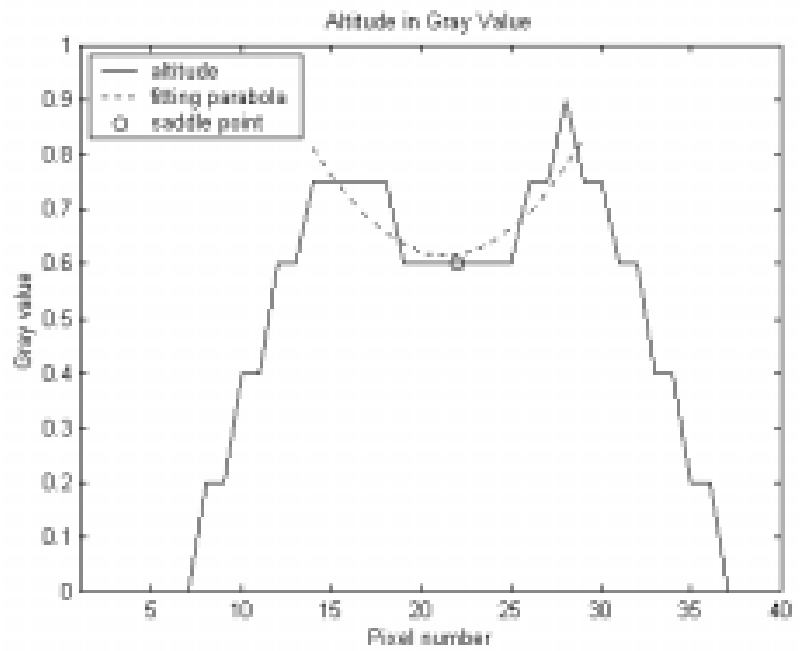

(a) altitude in plane $A$

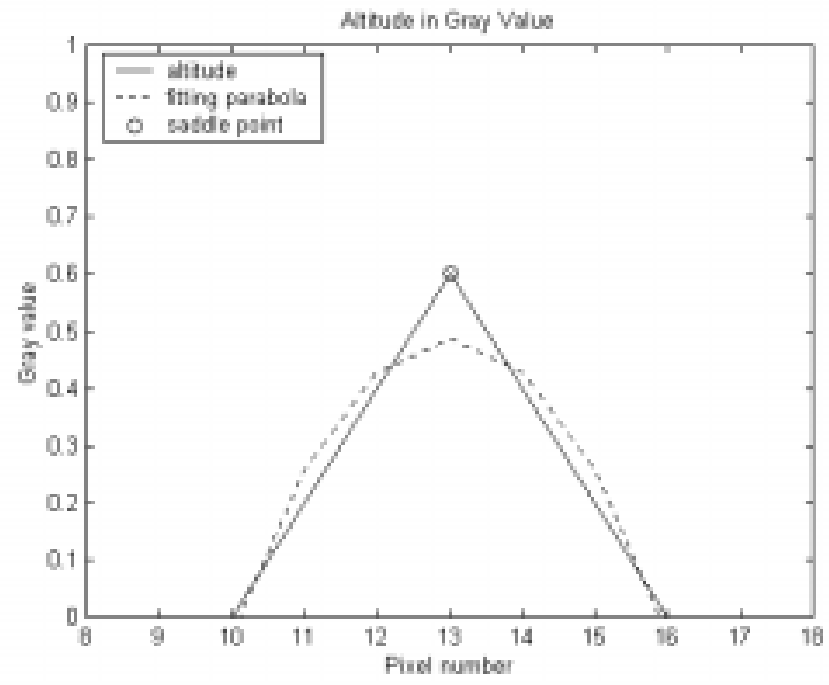

(b) altitude in plane $B$

Figure 5.6 Gray Level Altitude in Plane $A$ and $B$

\subsection{Detection of Saddle Point by Filtering}

\subsubsection{Filter Design}

A filter (or mask) can be designed to detect the saddle shaped domain and accordingly, the saddle point in the topographic surface. Based on finding saddle point, 
the cutting line can be oriented and separation can be carried out. Since the geometric characteristics of saddle surface are known, a filter was engineered to serve the separating purpose.

Again, hold the same definitions made for the plane $A$, plane $B$ and the set $\mathbf{A}$, set B, as stated in the last section. Further, let a filter have the size of $n \times m$, each grid holds value $f_{i j}, i=1,2, \ldots, n, j=1,2, \ldots, m$. Fig. 5.7 shows a filter of size $5 \times 5$.

\begin{tabular}{|l|l|l|l|l|}
\hline$f 11$ & $f 12$ & $f 13$ & $f 14$ & $f 15$ \\
\hline$f 21$ & $f 22$ & $f 23$ & $f 24$ & $f 25$ \\
\hline$f 31$ & $f 32$ & $\mathbf{f 3 3}$ & $f 34$ & $f 35$ \\
\hline f41 & $f 42$ & $f 43$ & $f 44$ & $f 45$ \\
\hline f51 & f52 & $f 53$ & $f 54$ & $f 55$ \\
\hline
\end{tabular}

Figure 5.7 A Filter of Size $5 \times 5$

The objective of designing a filter is to locate the saddle point. This requires that the filter can detect the gray value points distributing in a parabolic pattern in both planes $A$ and $B$. To achieve this, the value in each grid of the filter is assigned with +1 or -1 , symmetric about the reference point $\mathrm{f}_{i j}$ in planes $A$ and $B$. At each reference point, plane $A$ and $B$ are assumed to be orthogonal to each other, and may rotate simultaneously from $0^{\circ}$ to $90^{\circ}$ anti-clockwise searching for the orientation that qualifies the reference point to be the saddle point. If the preset conditions as given in the next section are met, the current reference point becomes the saddle point, and cutting then begins in the orientation of plane $B$. Fig. 5.8 demonstrates the values given for a filter of size $5 \times 5$, and the filter rotates from $0^{\circ}$ to $90^{\circ}$. 


\begin{tabular}{|c|c|c|c|c|}
\hline 0 & 0 & $+\mathbf{1}$ & 0 & 0 \\
\hline 0 & 0 & $\mathbf{+ 1}$ & 0 & 0 \\
\hline$+\mathbf{1}$ & $\mathbf{+ 1}$ & $\mathbf{0}$ & $\mathbf{- 1}$ & $\mathbf{- 1}$ \\
\hline 0 & 0 & $\mathbf{- 1}$ & 0 & 0 \\
\hline 0 & 0 & $\mathbf{- 1}$ & 0 & 0 \\
\hline
\end{tabular}

(a) filter at $0^{\circ}$

\begin{tabular}{|c|c|c|c|c|}
\hline 0 & $+\mathbf{1}$ & 0 & 0 & 0 \\
\hline 0 & $+\mathbf{1}$ & 0 & $\mathbf{- 1}$ & $\mathbf{- 1}$ \\
\hline 0 & 0 & $\mathbf{0}$ & 0 & 0 \\
\hline$+\mathbf{1}$ & $\mathbf{+ 1}$ & 0 & $\mathbf{- 1}$ & 0 \\
\hline 0 & 0 & 0 & $\mathbf{- 1}$ & 0 \\
\hline
\end{tabular}

(b) filter rotated by $22.5^{\circ}$

\begin{tabular}{|c|c|c|c|c|}
\hline+1 & 0 & 0 & 0 & $\mathbf{- 1}$ \\
\hline 0 & $+\mathbf{1}$ & 0 & $\mathbf{- 1}$ & 0 \\
\hline 0 & 0 & $\mathbf{0}$ & 0 & 0 \\
\hline 0 & $\boldsymbol{+ 1}$ & 0 & $\mathbf{- 1}$ & 0 \\
\hline+1 & 0 & 0 & 0 & $\mathbf{- 1}$ \\
\hline
\end{tabular}

(c) filter rotated by $45^{\circ}$

\begin{tabular}{|c|c|c|c|c|}
\hline 0 & 0 & 0 & $\mathbf{- 1}$ & 0 \\
\hline$+\mathbf{1}$ & $\mathbf{+ 1}$ & 0 & $\mathbf{- 1}$ & 0 \\
\hline 0 & 0 & $\mathbf{0}$ & 0 & 0 \\
\hline 0 & $\mathbf{+ 1}$ & 0 & $\mathbf{- 1}$ & $\mathbf{- 1}$ \\
\hline 0 & $\mathbf{+ 1}$ & 0 & 0 & 0 \\
\hline
\end{tabular}

(d) filter rotated by $67.5^{\circ}$

\begin{tabular}{|c|c|c|c|c|}
\hline 0 & 0 & $\mathbf{- 1}$ & 0 & 0 \\
\hline 0 & 0 & $\mathbf{- 1}$ & 0 & 0 \\
\hline $\mathbf{+ 1}$ & $\mathbf{+ 1}$ & $\mathbf{0}$ & $\mathbf{- 1}$ & $\mathbf{- 1}$ \\
\hline 0 & 0 & $\mathbf{+ 1}$ & 0 & 0 \\
\hline 0 & 0 & $\mathbf{+ 1}$ & 0 & 0 \\
\hline
\end{tabular}

(e) filter rotated by $90^{\circ}$

Figure 5.8 Rotation of Filter

\subsubsection{Saddle Point Conditions}

The filter demonstrated above can be extended to any larger size, and the rotating angle step then may be smaller accordingly. Suppose that in the plane $A$, there exist two points that are symmetrical to the reference point $S$ (recall that $S$ is also in the plane $B$ ). Let these two points denote $P_{A, R}$ and $P_{A, L}$ (subscript $R$ and $L$ indicate Right and Left to $S$ in the plane $A$ ), which take the gray value (altitude) $I_{A, R}$ and $I_{A, L}$, respectively. Correspondingly, assume that the filter values at these two locations are $f_{A, R}$ and $f_{A, L}$ (Note that if one is +1 , the other must be -1 ), respectively. 
Mathematically, if this reference point is detected as a saddle point $S$ which takes gray value $s_{i j}$, the following conditions hold:

In plane $A$, at certain orientation $\theta$ in the image plane,

$$
\begin{aligned}
& I_{A, R} f_{A, R}+I_{A, L} f_{A, L}=0 \\
& s_{i j}=\min \left\{P_{i j}, \forall P_{i j} \in \mathbf{A}\right\}
\end{aligned}
$$

Eqn. (5.3) ensures the two gray value points in plane $A$ have the same gray value, and Eqn. (5.4) indicates that the parabolic shape opens upward, and the reference point $S$ is at bottom on the parabola.

Similarly, in orthogonal plane $B$ at $\left(\theta \pm 90^{\circ}\right)$ in the image plane,

$$
\begin{aligned}
& I_{B, R} f_{B, R}+I_{B, L} f_{B, L}=0 \\
& s_{i j}=\max \left\{P_{i j}, \forall P_{i j} \in \mathbf{B}\right\}
\end{aligned}
$$

For the sake of clarity, two more characteristics may be observed,

$$
s_{i j} \in(\mathbf{A} \cap \mathbf{B})
$$

$$
(\mathbf{A}, \mathbf{B})^{\mathrm{c}}=(\mathbf{A} \cup \mathbf{B})-(\mathbf{A} \cap \mathbf{B})
$$

where $(\mathbf{A}, \mathbf{B})^{\mathrm{c}}$ denotes the complement set of sets $\mathbf{A}$ and $\mathbf{B}$.

The separation algorithm can be described as follows:

Step 1: At each reference point with $0^{\circ}$ orientation in plane $A$, first check its left and right neighboring pixels' gray value, see if all conditions set in Eqn. (5.3) and (5.4) are satisfied. If not, then extend to next pair of pixels before and after the two pixels just checked in the same plane. Same process is taken until the conditions are satisfied within the preset filter size $n$.

Step 2: If the conditions are met during searching within the preset filter size $n$, then further check its upper and lower neighboring pixels in the orthogonal plane $B$, see if 
all conditions set in Eqn. (5.5) and (5.6) are satisfied. If not, check next pair of pixels' gray value extending upward and downward within the preset filter size $m$.

Step 3: If an affirmative answer has been obtained for step 1 and 2, in other words, if logical $A N D$ is used to combine the two conditions set in step land step 2, and a positive answer is found, then the current reference point is the desired saddle point. Followed is to carry out the cutting process in the direction of plane B. Step 1 to Step 3 are repeated for the next new reference point in the image.

Step 4: If a negative answer has been found in either step 1 or step 2, in other words, the AND logic fails, then the current reference point is not the saddle point at the current orientation, stop.

Step 5: Simultaneously rotate the plane $A$ and plane $B$ to certain orientation, repeat step1 to step 5.

Step 6: If after a rotation of $90^{\circ}$ is finished, and no $A N D$ logic is found affirmative, then the current reference point is detected as a non-saddle-point. Move to the next new reference point, repeat Step 1 to Step 6.

The simplified pseudocode of separation for touching and overlapping shapes is listed as follows: 
/*A gray scale ultimately eroded image $\mathbf{G}$ is available, and a filter of size $n \times m$ is selected.*/

/*Scan with the filter */

for (each reference point)

if (within the filter size $n$ in plane $A$, all conditions are met, referring to Eqn. (5.3)and (5.4)) $A N D$ (within the filter size $m$ in plane $B$, all conditions are met, referring to Eqn. (5.5) and (5.6))

- the current reference point is a saddle point

- cut in the direction of plane $B$

else

- simultaneously rotate both plane $A$ and $B$ anti-clockwise to certain angle, provided a total $90^{\circ}$ is not swept. Otherwise stop.

- go back to above "if" condition, check $A N D$ logic again in new orientation just rotated. end

end /* end of "for" loop */

Referring to Fig. 5.5 (a), the separated shapes are shown in Fig. 5.9 (b), while (a) shows the connected shapes.

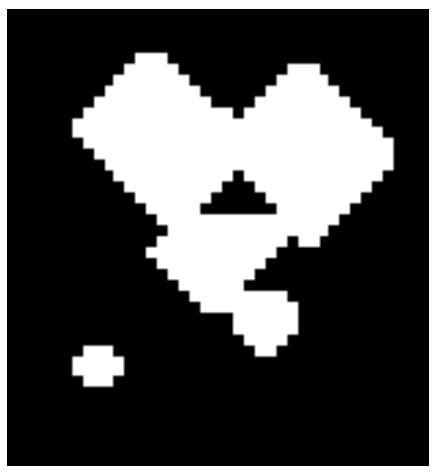

(a) connected shapes

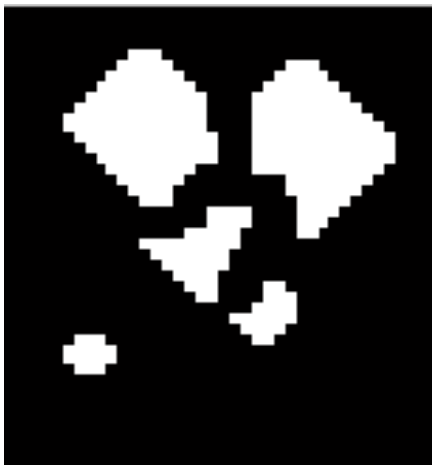

(b) separated shapes

Figure 5.9 Separation by Filtering

\subsection{Testing on a Real Image}

Now, using a structuring element of size $3 \times 3$ for sequential erosion, and a filter of size $15 \times 15$ for saddle point detection, the touching and overlapping limestone rocks 
digitized in a $484 \times 512$ matrix, as shown in Fig. 5.10 (a), was separated. The corresponding binary image is given in sub-figure (b). A total of 10 erosion operations has been elapsed before the ultimate erosion is reached. Sub-figure (c) demonstrates the resultant topographic surface, while (d) portraits the same topographic surface in an oblique view. Finally, an image of separated particles is presented in sub-figure (e).

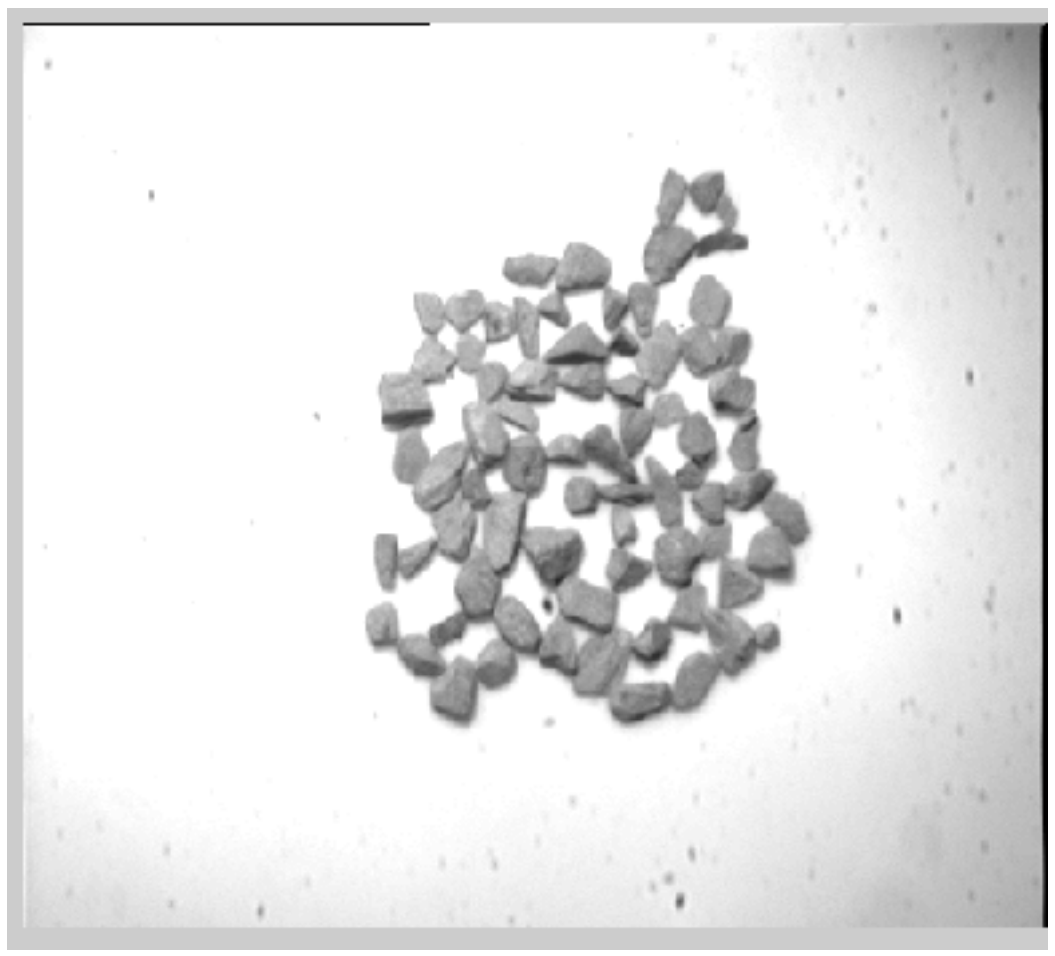

(a) gray scale image 


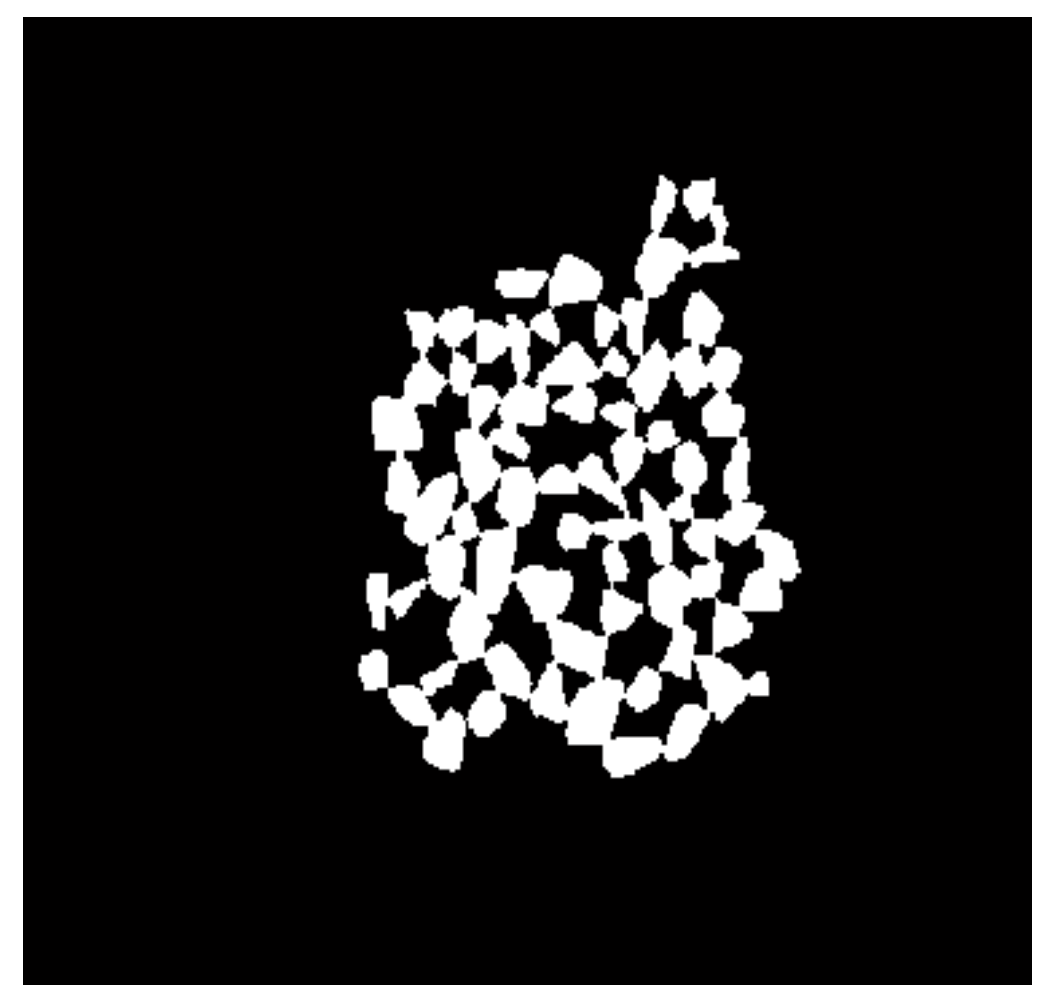

(b) binary image

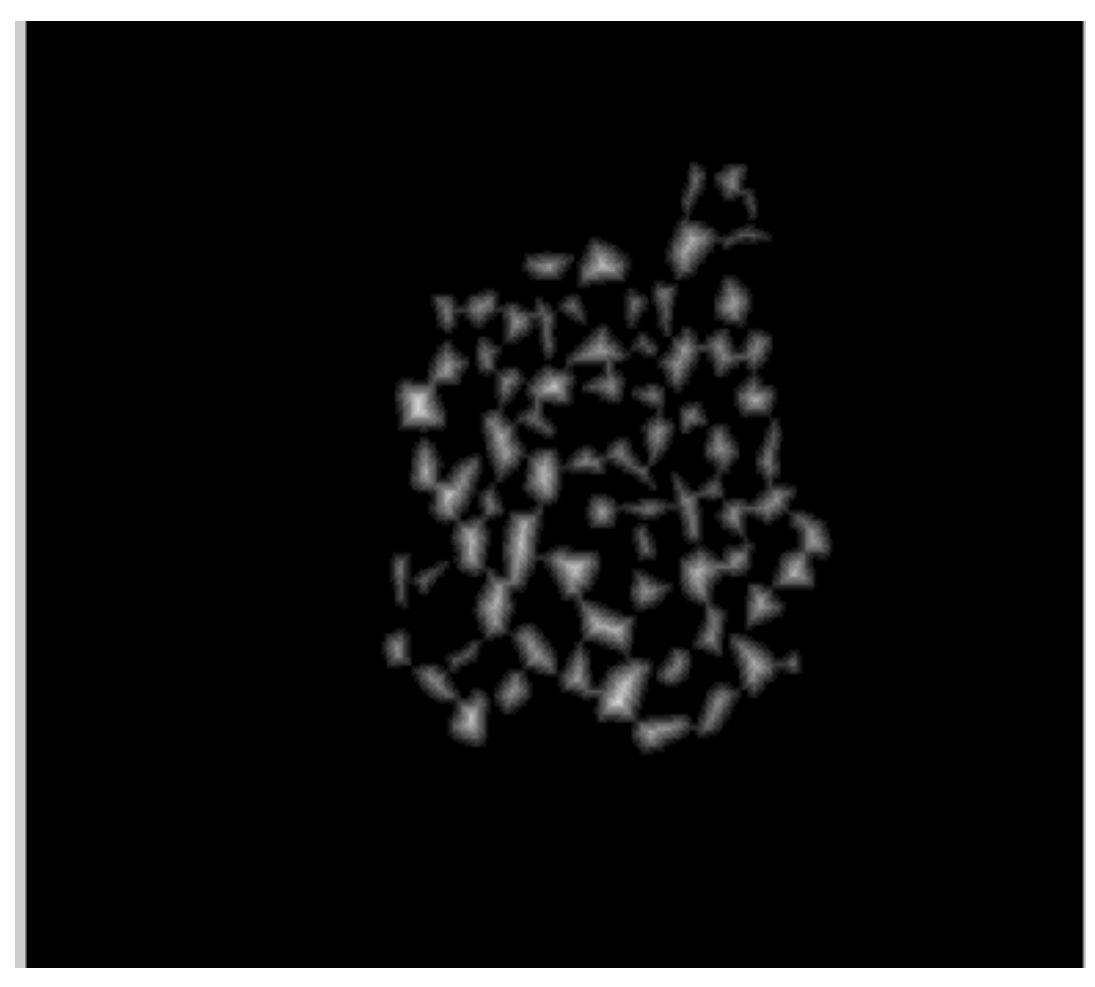

(c) topographic surface (Top view) 


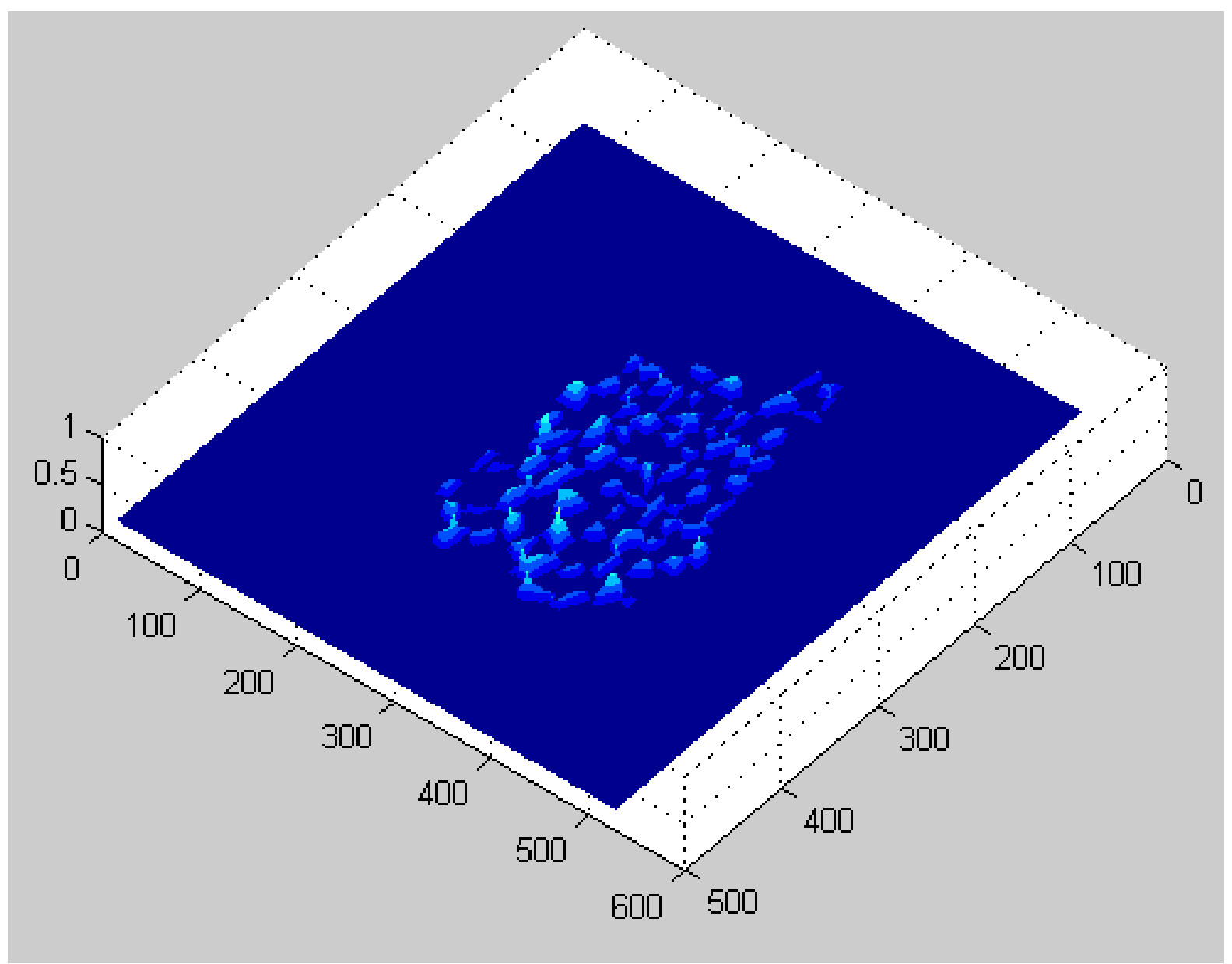

(d) topographic surface (Oblique view) 


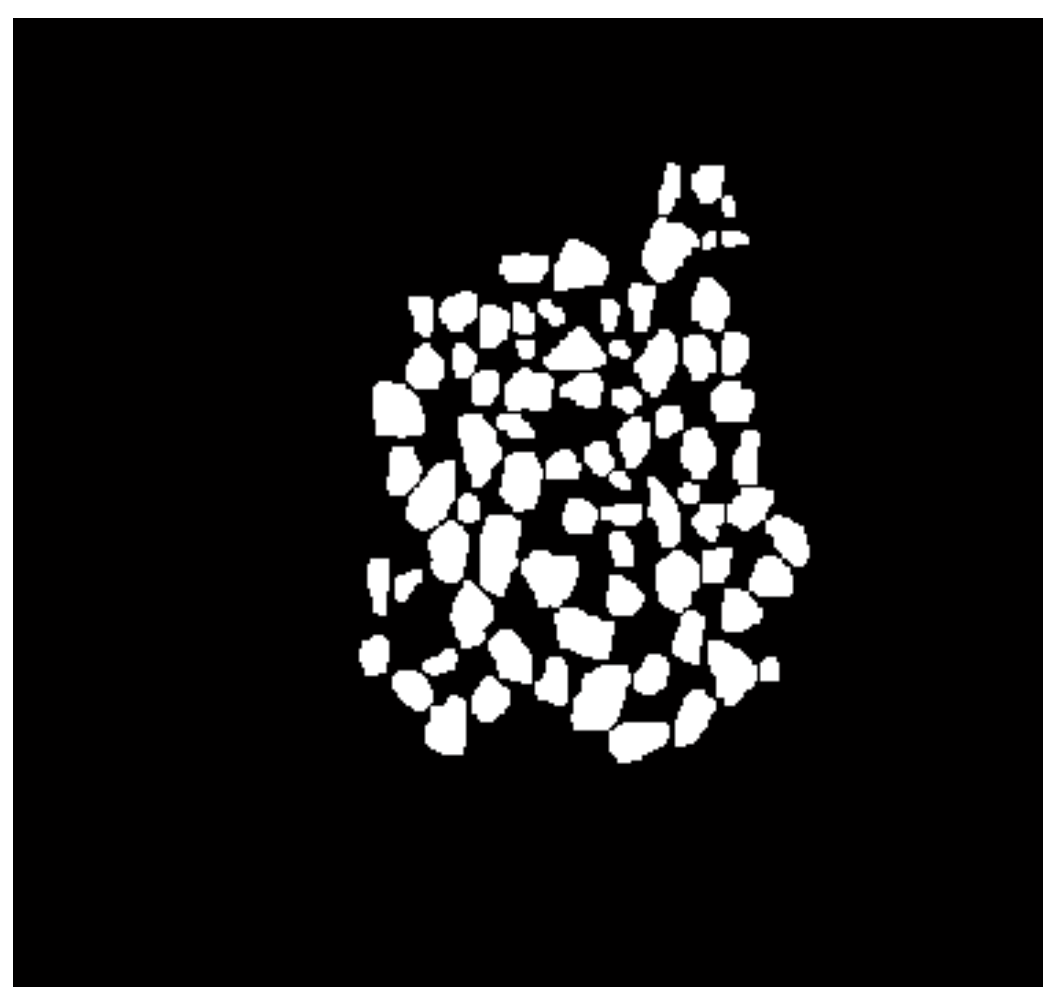

(e) particle separated image

Figure 5.10 Separation Process

The size of the filter used in the separation process is problem dependent. Larger filters give more accurate detection, but take longer to perform the filtering. Proper size can be determined experimentally. Some "false cutting" can occur for particles whose edge contour is significantly concave on opposite edges. 


\section{VOLUMETRIC MODELING}

\subsection{Introduction}

Particle size distribution (gradation) in the Superpave and in many other applications is based on the sieve mesh sizes and particle mass. In most sieving operations, gradation is evaluated as "percent-passing" by mass. The minor diameter of the particle profile shape obtained through using principal eigen analysis is used as a particle's size. This dimension is correlated to the sieve size. In this research, the density is assumed a constant, so the total mass is proportional to the volume of aggregates. Also in this research, all the obtainable information comes from a 2-D image. Volume information is not directly observed. Lack of 3-D information brings the need to estimate the volume using some of the available 2-D measurements, such as area, aspect ratio, signature mean and variance. In other words, modeling the volume of aggregates is needed.

Statistical techniques are important for image analysis. From the images captured of the mineral aggregates, all the sizes and shapes are random. The process of extracting useful information becomes equivalent to estimating random variables. The objective in this chapter is to estimate the volume of mineral aggregates to serve the purpose of gradation.

\subsection{Modeling Sample Preparation}

To observe how particle mass might be correlated with some of its parameters found in 2-D images, some samples of aggregate were collected. Fig. 6.1 demonstrates images of some of these samples. Altogether 501 pieces of limestone rock varying in 
size and shape were imaged. The following measurements were taken for each individual particle:

- Projected area $\left(\mathrm{mm}^{2}\right)$

- Major and minor diameter $(\mathrm{mm})$

- Aspect ratio

- Non-normalized signature mean $(\mathrm{mm})$ and variance $\left(\mathrm{mm}^{2}\right)$

- Mass $(g)$ and volume $\left(\mathrm{mm}^{3}\right)$ with a measured constant density of of 0.00305 $\left(\mathrm{g} / \mathrm{mm}^{3}\right)$

The methods for obtaining above measurements except mass and volume were described in Chapter 4. The mass of each particle was found using a laboratory digital balance, and added to the data vector. All the measurements are listed in Appendix I. The aggregates were arranged on the lighting box as shown in Fig. 6.1 so that each particle could be paired with its image statistics. The particles' sizes are visually judged ranging from $4.75(\mathrm{~mm})$ to $25.00(\mathrm{~mm})$.

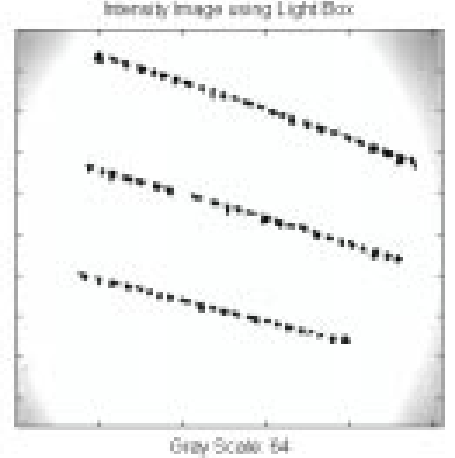

(a) size $\approx 4.75(\mathrm{~mm})$

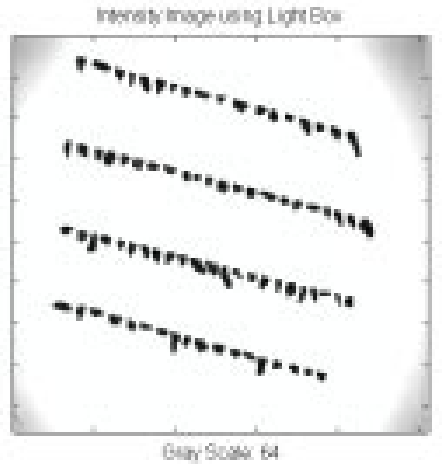

(b) size $\approx 9.50(\mathrm{~mm})$ 


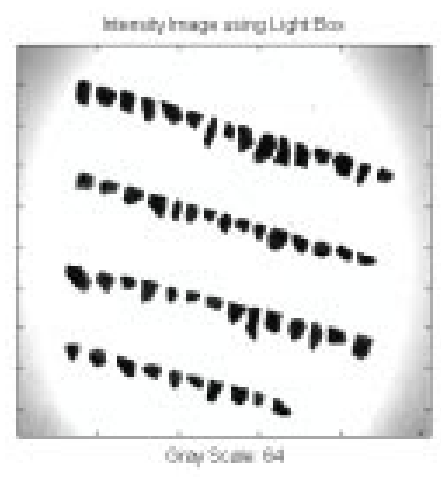

(c) size $\approx 12.50(\mathrm{~mm})$

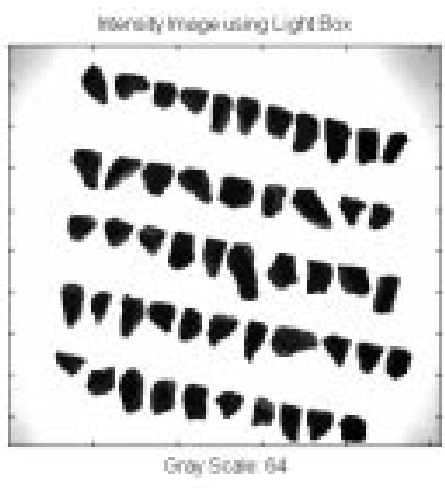

(d) size $\approx 19.00 \sim 25.00(\mathrm{~mm})$

Figure 6.1 Sample Photos

To gain some intuitive perceptions about how some parameters from a particle's 2-D image influence its mass, Fig. 6.2 illustrates the observations in which the mass of each individual particle is plotted against its area, aspect ratio, non-normalized signature mean and variance, major and minor diameter, respectively.

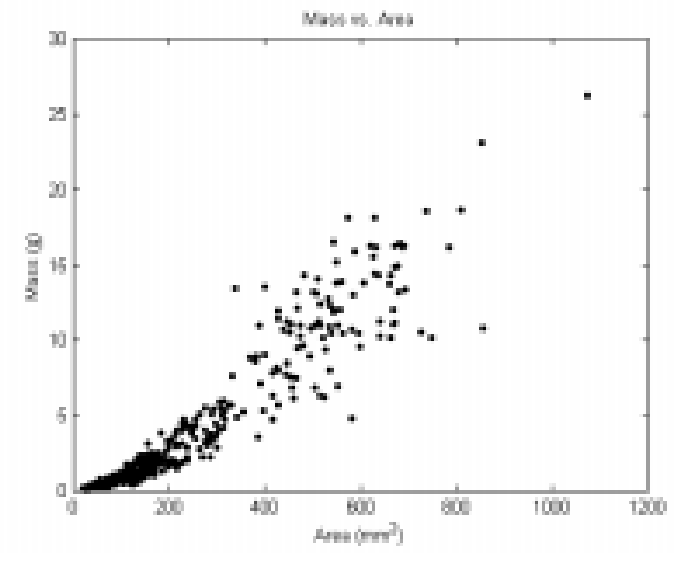

(a) mass vs. area

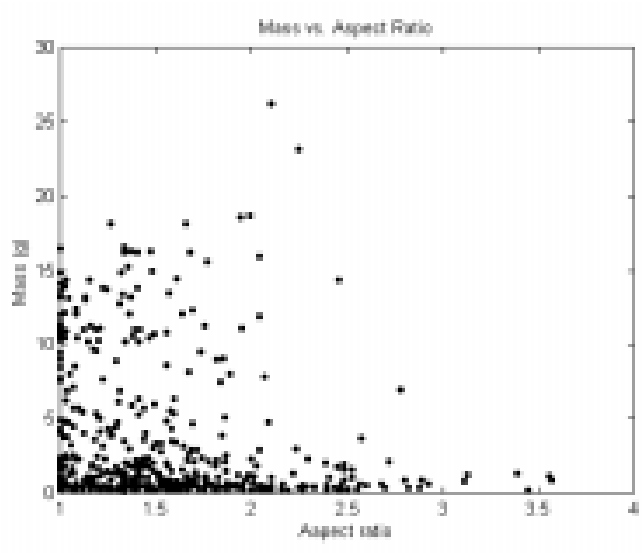

(b) mass vs. aspect ratio 


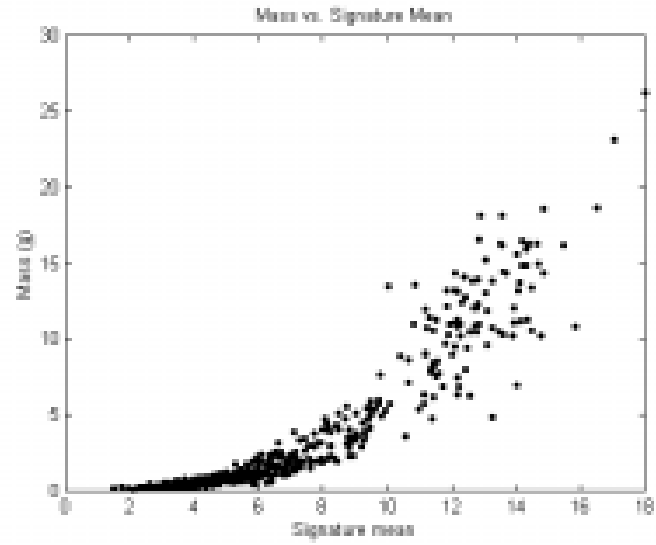

(c) mass vs. signature mean

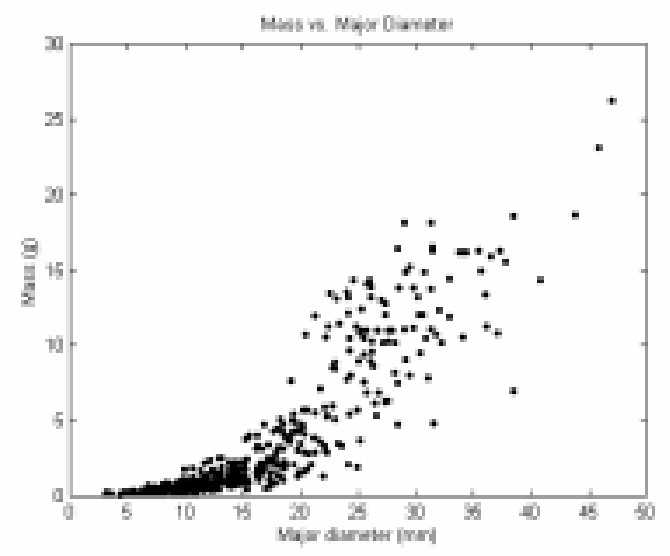

(e) mass vs. major diameter

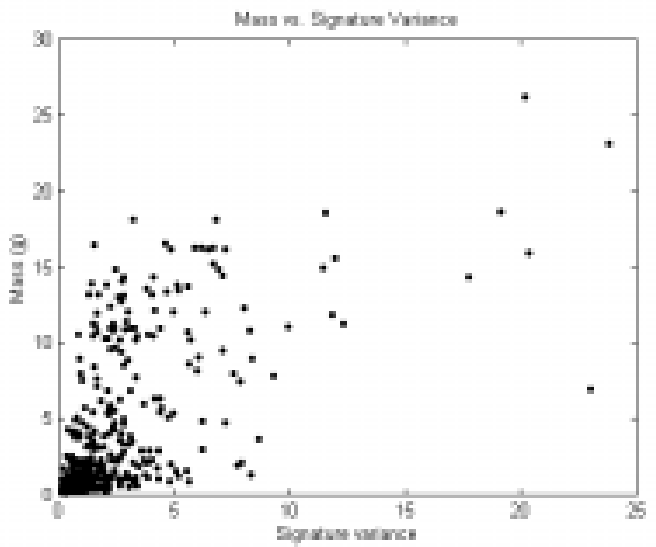

(d) mass vs. signature variance

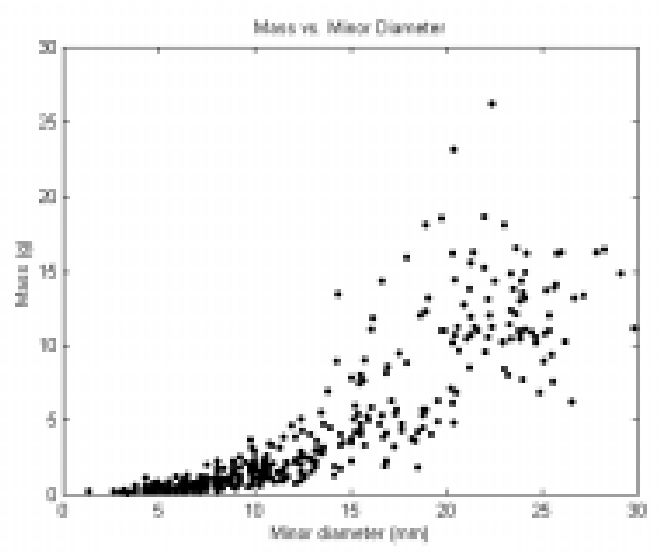

(f) mass vs. minor diameter

\section{Figure 6.2 Sample Data Observations}

If coupled with shape information, the particle's projected area can reflect its size. The signature mean is basically the "effective radius". The projected area and signature mean are apparently the dominant factors that correlate with the mass from both intuitive and experimental considerations. The general trend shows that particle mass value grows as its area and signature mean increase. The aspect ratio gives partial information about the profile shape, but is obviously not correlated directly with mass, at least in this 
sample. The signature variance may reveal how irregular the particle shape is, more specifically, how far the actual edge line deviates from the circumference whose radius is the signature mean. Major and minor diameter also indicate particle size.

Evidently, discovering a way to more accurately predict particle's 3-D measurements should be attempted based on combinations of several pertinent 2-D variables. The objective here must be to create a model using appropriate measurements from the image data, so that a "good" model is obtained. A good model will be the one in which the estimated particle volumes and the actual particle volumes are equal, at least when averaged over sufficient number of particles.

\section{3 $\underline{\text { Model Selection }}$}

Regression analysis is a statistical technique for modeling the relationship between two or more variables. Once an adequately good model is found, it is then used to predict the response to the new set of variables of the same type. In fact, multiple regression is one of the most widely used statistical techniques [16].

In general, for a dependent variable, or response, $y$ that may be related to $k$ independent variables, the standard multiple linear regression (MLR) model takes form

$$
y=\beta_{0}+\beta_{1} x_{1}+\beta_{2} x_{2}+\ldots+\beta_{k} x_{k}+\varepsilon
$$

where

$y$ : Observation or response.

$\beta_{j}:$ Partial regression coefficient, $j=0,1,2, \ldots k$

$x_{j}:$ Regressor variable, $j=1,2, \ldots k$

$\varepsilon:$ Random error with zero mean and variance $\sigma^{2}$. 
This model describes a hyperplane in the $k$-dimensional space of the independent variables $\left\{x_{j}\right\}$. The parameter $\beta_{j}$ represents the expected change in the response y per unit change in $x_{j}$ when all the remaining independent variables $x_{i}(\mathrm{i} \neq \mathrm{j})$ are held constant.

It is worth mentioning that, in general, any regression model that is linear in the parameters, that is, the $\beta$ values, is a linear regression model. Models that are polynomial or include interaction effects in the parameters may also be analyzed by multiple linear regression model by defining regressor variables that include the nonlinear effects [16].

In matrix notation, the model in Eqn. (6.1) can be expressed as

$$
\mathbf{Y}=\mathbf{X} \boldsymbol{\beta}+\varepsilon
$$

Where

$$
\begin{array}{ll}
\mathbf{Y}=\left[\begin{array}{c}
y_{1} \\
y_{2} \\
\vdots \\
y_{n}
\end{array}\right] & \mathbf{X}=\left[\begin{array}{ccccc}
1 & x_{11} & x_{12} & \cdots & x_{1 k} \\
1 & x_{21} & x_{22} & \cdots & x_{2 k} \\
1 & x_{31} & x_{32} & \cdots & x_{3 k} \\
\vdots & \vdots & \vdots & & \vdots \\
1 & x_{n 1} & x_{n 1} & \cdots & x_{n k}
\end{array}\right] \\
\boldsymbol{\beta}=\left[\begin{array}{c}
\beta_{1} \\
\beta_{2} \\
\vdots \\
\beta_{k}
\end{array}\right] & \boldsymbol{\varepsilon}=\left[\begin{array}{c}
\varepsilon_{1} \\
\varepsilon_{2} \\
\vdots \\
\varepsilon_{n}
\end{array}\right]
\end{array}
$$

The summation of error square is

$$
L=\sum_{i=1}^{n} \varepsilon_{i}^{2}=\boldsymbol{\varepsilon}^{\mathrm{T}} \boldsymbol{\varepsilon}=(\mathbf{Y}-\mathbf{X} \boldsymbol{\beta})^{\mathrm{T}}(\mathbf{Y}-\mathbf{X} \boldsymbol{\beta})
$$


The vector of least square estimators, $\hat{\mathbf{a}}$, can be obtained by minimizing $L$ and satisfying

$$
\left.\frac{\partial L}{\partial \hat{\mathbf{a}}}\right|_{\hat{\hat{a}}}=-2 \mathbf{X}^{\mathrm{T}} \mathbf{Y}+2 \mathbf{X}^{\mathrm{T}} \mathbf{X} \hat{\mathbf{a}}=\mathbf{0}
$$

thus

$$
\hat{\mathbf{a}}=\left(\mathbf{X}^{\mathrm{T}} \mathbf{X}\right)^{-1} \mathbf{X}^{\mathrm{T}} \mathbf{Y}
$$

The estimated regression model is

$$
\hat{\mathbf{Y}}=\mathbf{X} \hat{\mathbf{a}}
$$

\subsection{Particle Geometric Assumptions and Definitions}

To use MLR model, the regressor variables that may influence the observations must be found. Reasonably, these variables are particle geometry related.

Referring to Fig. 6.3, some important variables that will relate to the prospective MLR model are defined:

$\mathrm{H}_{\text {top }}$ : Top height, which is measured from the imaged background up to the highest point found in the upper surface of the particle.

$\mathrm{H}_{\text {cent }}$ : Central height, which is also measured from the imaged background and through the centroid of the profile shape to the upper surface.

$\mathrm{H}_{\text {nom }}$ : Nominal height, which is the most related variable. The value of this height quantitatively results from the actual volume of the particle, as illustrated in sub-figure (a), divided by its projected area, as depicted in sub-figure (c). In other words, the actual volume of any particle can be obtained by multiplying its projected area with its nominal height.

$\mathrm{D}_{\text {maj }}$ : Major diameter of the particle's profile shape. 
$\mathrm{D}_{\min }$ : Minor diameter of the particle's profile shape.

\section{$\downarrow^{\text {Viening }}$ direction}

(a) actual

shape and volume

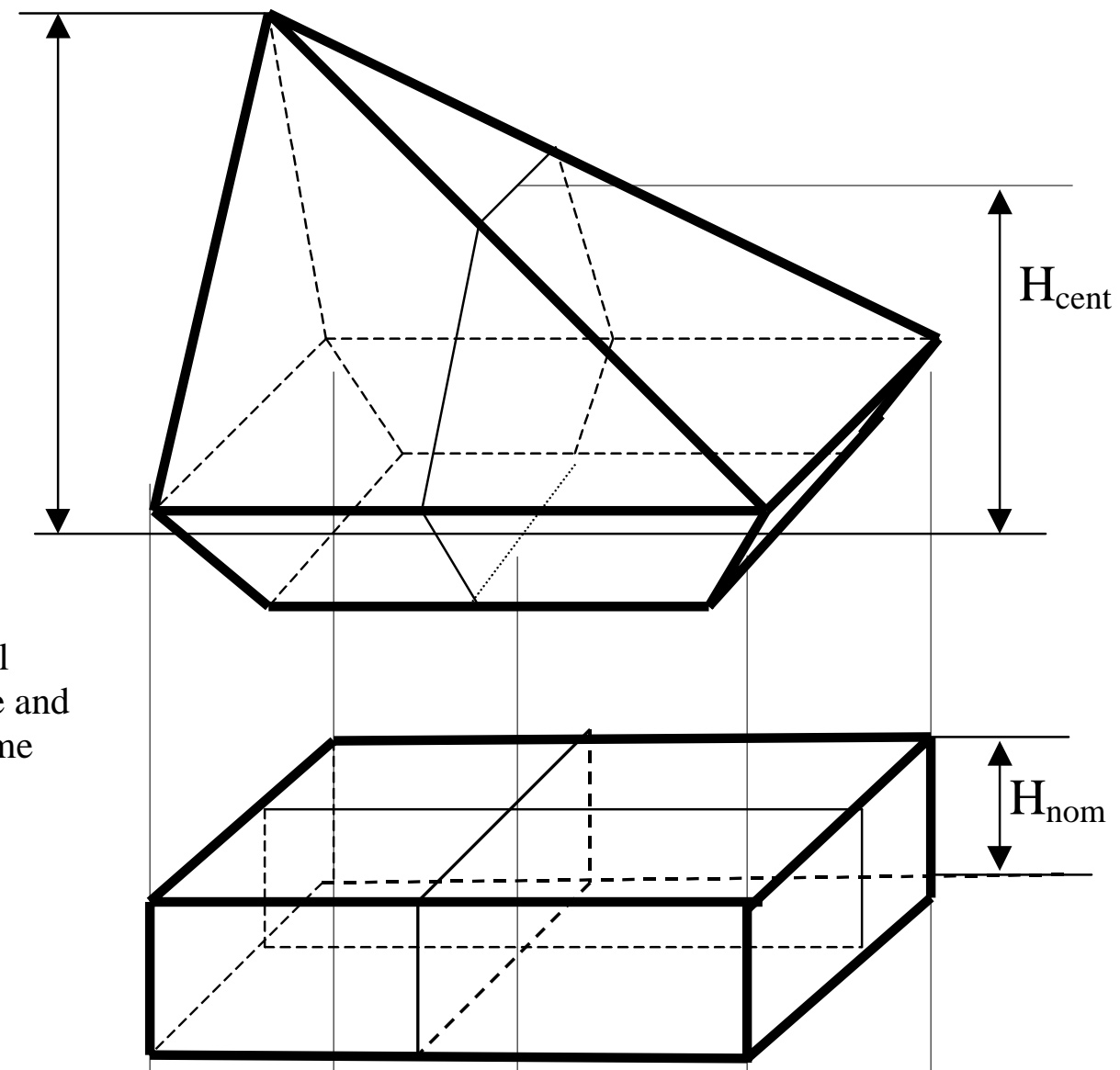

(b) equivalent shape and volume

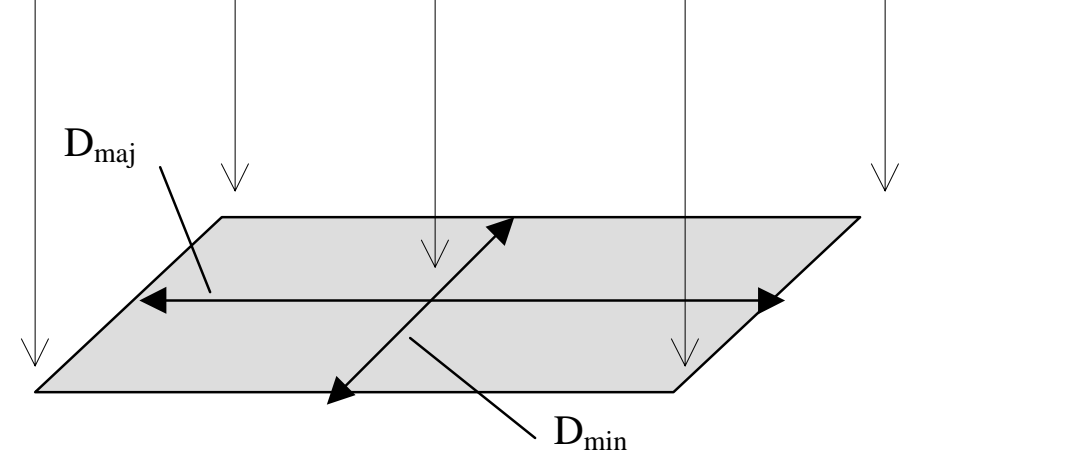

(c) common projected area

Figure 6.3 Geometric Perspective of Particle 
It is clear that the posture of the particles in the imaging scene may affect the modeling results. Here the following assumptions regarding the pattern of particle positioning in the imaged scene are claimed:

1) After randomly vibrating and toppling, the majority of the particles will lie on the background with their maximum projected area facing down. This state is termed "stable state" hereafter.

2) All particles are assumed to have a constant density.

The assumption 1) can lead to the following inequalities:

For any geometric objects, at the stable state

$$
0<H_{\text {nom }} \leq H_{\text {cent }} \leq H_{\text {top }} \leq D_{\text {min }}
$$

While for the most crushed limestone aggregates at the stable state, it holds

$$
0<H_{\text {nom }} \leq D_{\min }
$$

thus

$$
0<\frac{H_{\text {nom }}}{D_{\text {min }}} \leq 1
$$

The term $H_{\text {nom }}$ is defined as the flatness of particle throughout this work.

$D_{\text {min }}$

\subsection{MRL Model Building}

Flatness of the particle is suspected to relate to some geometric attributes, such as elongation, roundness of the profile shape, and jaggedness of the edge line. All the mentioned variables are defined to be particle size and volume independent. 
Following MLR model is build first, then each variable in it is described thereafter.

$$
\frac{H_{n o m}}{D_{\text {min }}}=\beta_{0}+\beta_{1} \frac{D_{m a j}}{D_{\text {min }}}+\beta_{2} \frac{D_{\text {min }}}{2 M_{\text {sig }}}+\beta_{3}\left(-\log \left(\frac{V_{s i g}}{M_{s i g}^{2}}\right)\right)^{-1}+\varepsilon
$$

where

$H_{\text {nom }}$ : Nominal height $(\mathrm{mm})$.

$D_{\min }$ : Minor diameter $(\mathrm{mm})$.

$D_{m a j}:$ Major diameter $(\mathrm{mm})$, also as defined in Chapter 4.

$M_{\text {sig }}$ : Mean value of the non-normalized signature $(\mathrm{mm})$.

$V_{\text {sig }}$ : Variance of the non-normalized signature $\left(\mathrm{mm}^{2}\right)$.

$\varepsilon \quad$ : Random error.

Definition and interpretation of each variable in Eqn. (6.10) are as follows:

$\frac{H_{n o m}}{D_{\min }}:$ Flatness, as illustrated in Fig. 6.4 (a).

$\frac{D_{m a j}}{D_{\min }}$ : Elongation. It takes the value of aspect ratio, as depicted in Fig. 6.4 (b).

$\frac{D_{\min }}{2 M_{s i g}}:$ Roundness. It measures the circularity of the particle. Its value increases to 1 as the profile shape of the particle becomes more circular. See Fig. $6.4(\mathrm{c})$.

$\frac{V_{s i g}}{M_{s i g}^{2}}$ : Jaggedness. For a perfectly smooth edge line, the value is zero, as demonstrated in Fig. 6.4 (d). 

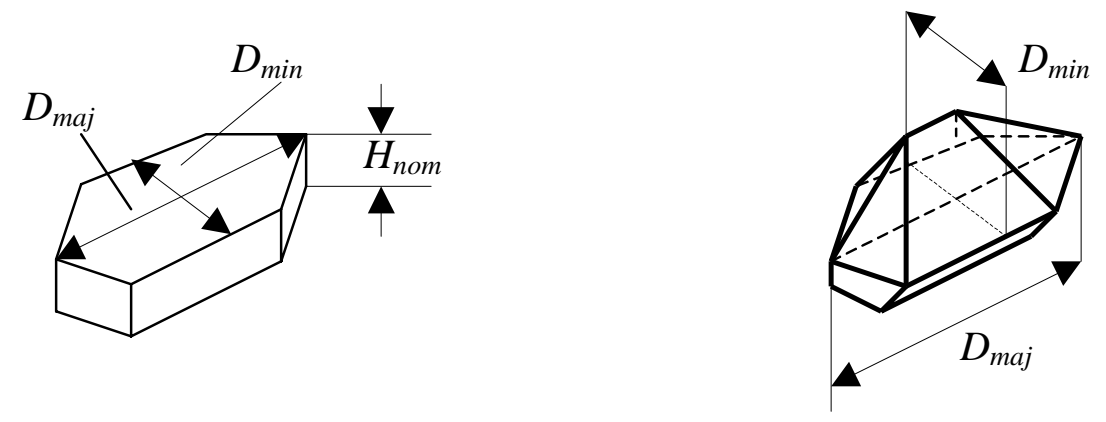

(a) flatness: $\frac{H_{n o m}}{D_{\min }}$

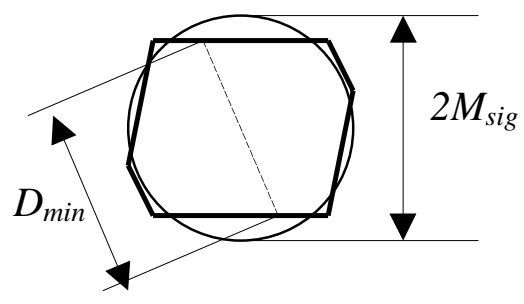

(c) roundness: $\frac{D_{\min }}{2 M_{s i g}}$ (b) elongation: $\frac{D_{m a j}}{D_{\min }}$

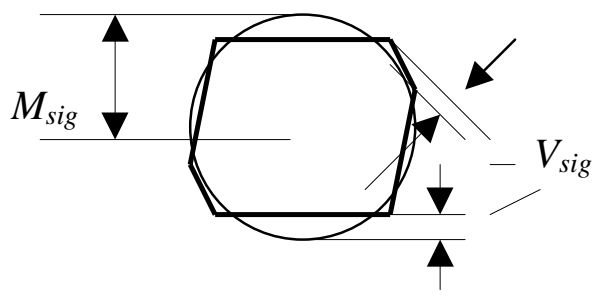

(d) jaggedness: $\frac{V_{s i g}}{M_{s i g}^{2}}$

\section{Figure 6.4 Interpretation of Model Variables}

Some aspects regarding $\frac{V_{\text {sig }}}{M_{\text {sig }}^{2}}$ need to be mentioned here: first, the signature mean is squared in the denominator to make the parameter dimensionless. Second, the logarithm transformation of $\frac{V_{\text {sig }}}{M_{\text {sig }}^{2}}$ makes the very small ratio values more readable and offsets the possible inaccuracy caused by numerical truncation in the computer system. Third, the reciprocity of the negative logarithm transformation ensures a positive value that decreases to zero as the edge line becomes smoother. To clearly demonstrate the 
purpose of this data transformation, given a array of small (not very small in order to view the plots effectively) values, starting from 0.01 up to 0.33 , increasing step by 0.04 , Fig. 6.5 shows values of the logarithm transformation of this array, and the final values of the negative reciprocity of this transformation.

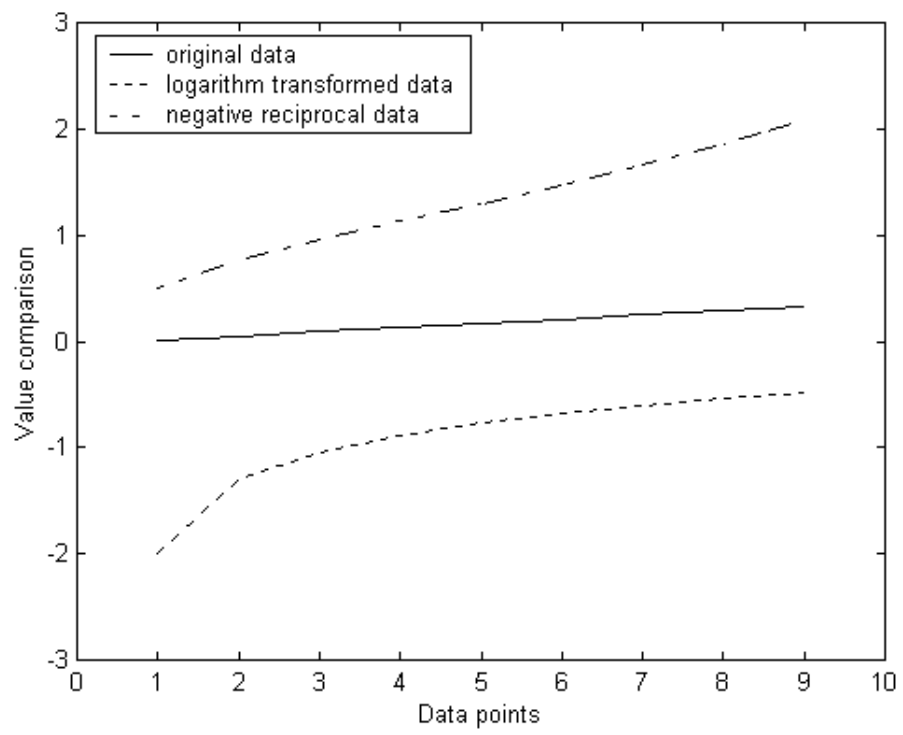

Figure 6.5 Data Transformation

The model in Eqn. (6.10) can be simplified, correspondingly, as

$$
Y_{\text {flat }}=\beta_{0}+\beta_{1} X_{\text {elong }}+\beta_{2} X_{\text {round }}+\beta_{3} X_{\text {jagged }}+\varepsilon
$$

where $X_{\text {elong }}, X_{\text {round, }}$, and $X_{\text {jagged }}$ are equivalent to the corresponding regressor variables in Eqn. (6.10).

In matrix notation,

$$
\mathbf{Y}_{\text {flat }}=\hat{\mathbf{a}} \mathbf{X}+\varepsilon
$$

The unbiased regression coefficient estimators are thus in matrix form

$$
\hat{\mathbf{a}}=\left(\mathbf{X}^{\mathrm{T}} \mathbf{X}\right)^{-1} \mathbf{X}^{\mathrm{T}} \mathbf{Y}_{\text {flat }}
$$


The prediction of flatness for each particle is

$$
\hat{Y}_{\text {flat }}=\hat{\beta}_{0}+\hat{\beta}_{1} X_{\text {elong }}+\hat{\beta}_{2} X_{\text {round }}+\hat{\beta}_{3} X_{\text {jagged }}
$$

where

$$
\hat{Y}_{\text {flat }}=\frac{\hat{H}_{\text {nom }}}{D_{\min }}
$$

Recall that the final goal is to use this model to predict the volume of the particle. Since the volume estimate used in this research equals the projected area multiplied by corresponding nominal height, the following expression holds:

$$
\hat{V}=\hat{H}_{\text {nom }} A_{\text {part }}=\hat{Y}_{\text {flat }} D_{\text {min }} A_{\text {part }}
$$

where

$$
A_{\text {part }} \text { : Particle projected area }\left(\mathrm{mm}^{2}\right) \text {. }
$$

\subsection{Overall Modeling Effect}

Using all the available data from the modeling sample of 501 particles, the complete model in Eqn. (6.10) was found to be:

$$
\frac{\hat{H}_{n o m}}{D_{\min }}=0.6660-0.1390 \frac{D_{m a j}}{D_{\text {min }}}-0.4754 \frac{D_{\text {min }}}{2 M_{s i g}}+0.4668\left(-\log \left(\frac{V_{s i g}}{M_{s i g}^{2}}\right)\right)^{-1}
$$

From Eqn. (6.14) and Eqn. (6.15), Eqn. (6.16) can be rewritten as

$$
\hat{V}=\hat{\beta}_{0} A_{\text {part }} D_{\text {min }}+\hat{\beta}_{1} A_{\text {part }} D_{\text {min }} X_{\text {elong }}+\hat{\beta}_{2} A_{\text {part }} D_{\text {min }} X_{\text {round }}+\hat{\beta}_{3} A_{\text {part }} D_{\text {min }} X_{\text {jagged }}
$$

Now, further define 


$$
\begin{aligned}
& X_{0}^{*}=A_{\text {part }} D_{\text {min }} \\
& X_{\text {elong }}^{*}=A_{\text {part }} D_{\text {min }} X_{\text {elong }} \\
& X_{\text {round }}^{*}=A_{\text {part }} D_{\text {min }} X_{\text {round }} \\
& X_{\text {jagged }}^{*}=A_{\text {part }} D_{\text {min }} X_{\text {jagged }}
\end{aligned}
$$

Thus, Eqn. (6.18) becomes

$$
\hat{V}=\hat{\beta}_{0} X_{0}^{*}+\hat{\beta}_{1} X_{\text {elong }}^{*}+\hat{\beta}_{2} X_{\text {round }}^{*}+\hat{\beta}_{3} X_{\text {rough }}^{*}
$$

with the same $\hat{\mathbf{a}}$ values as obtained.

Fig. 6.6 shows the overall modeling effect, i.e., comparison of estimated and true volume. Fig. 6.7 zooms in on the constituent parts of the plot in Fig. 6.6 (b) for better viewing.

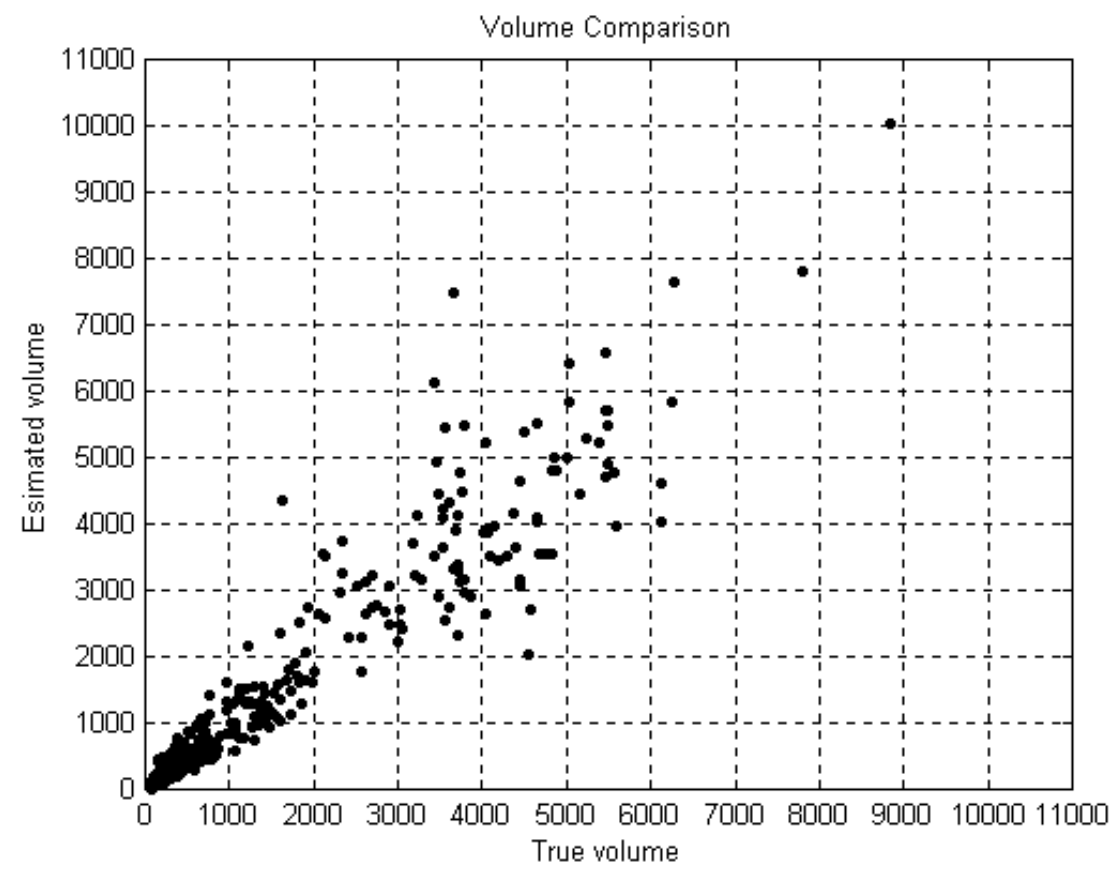

(a) 


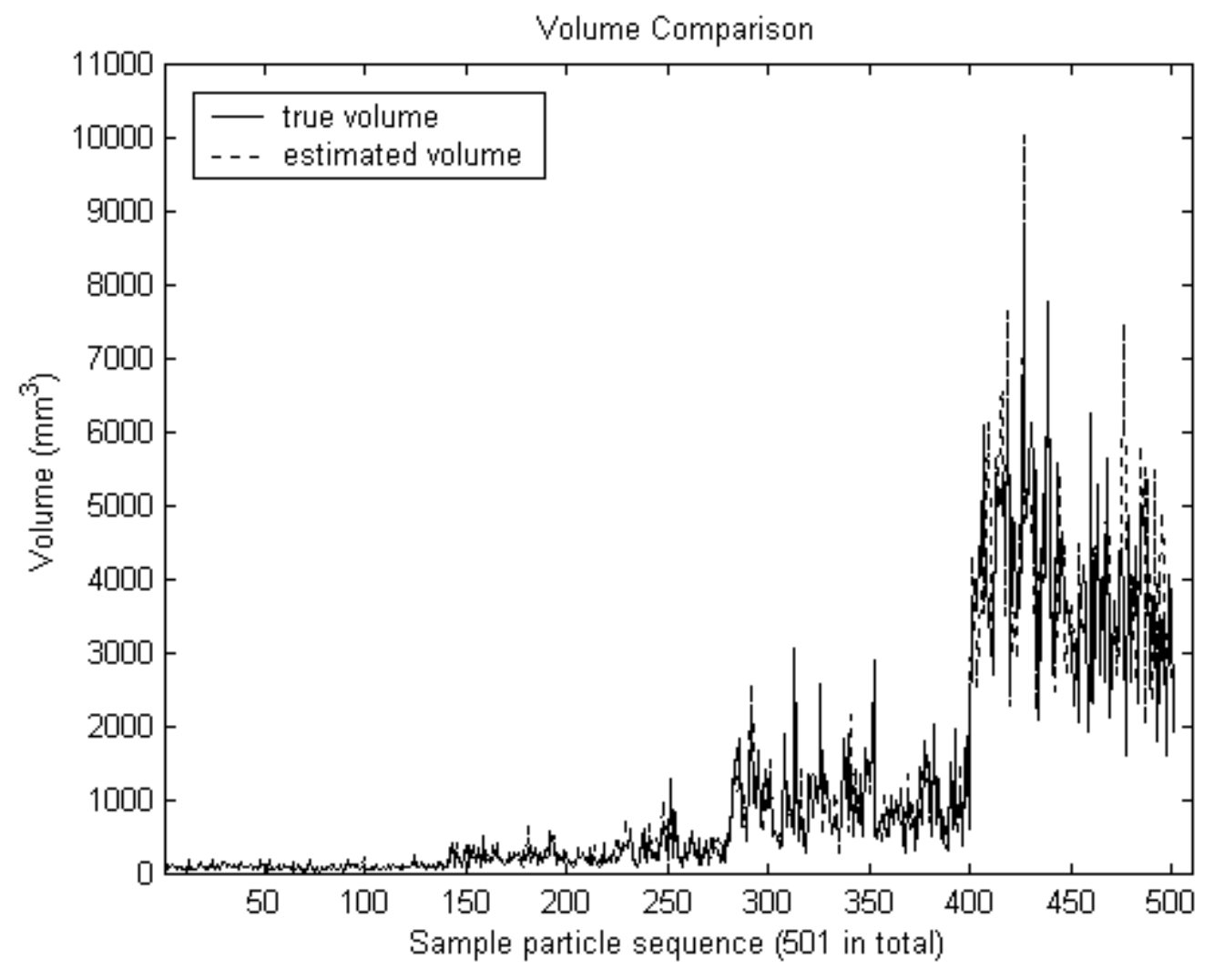

(b)

Figure 6.6 Volume Comparison

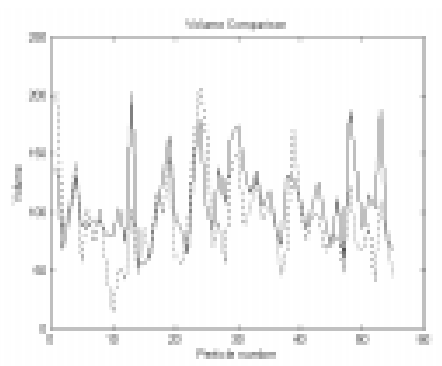

(a) particle \#1 \#55

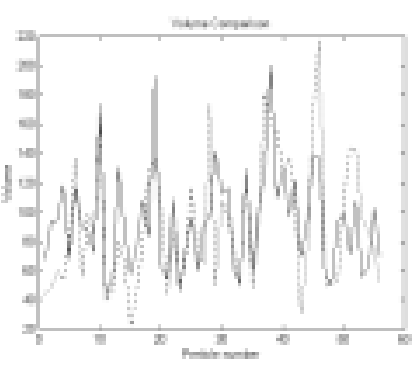

(b) particle \#56 \#110

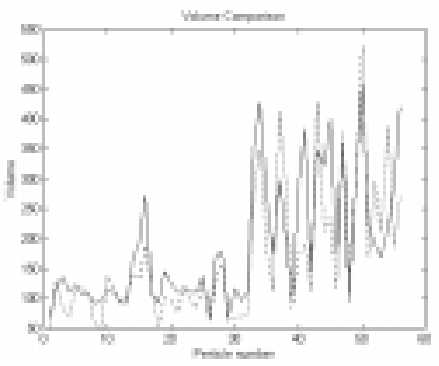

(c) particle \#111 \#165 


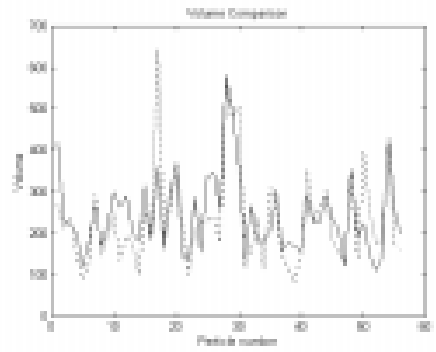

(d) particle \#166 \#220

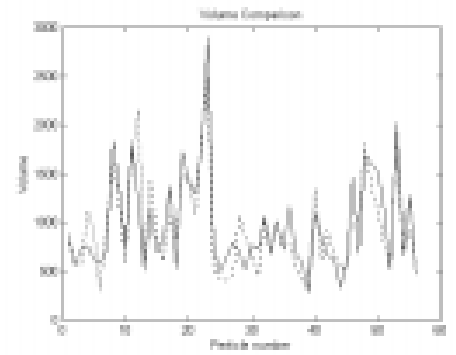

(g) particle \#331 \#385

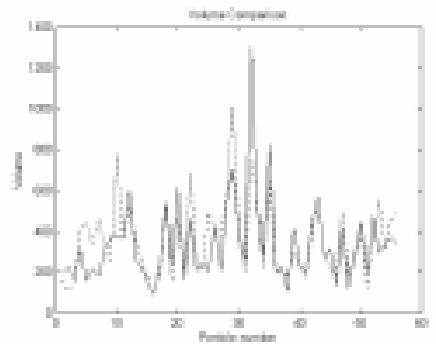

(e) particle \#221 \#275

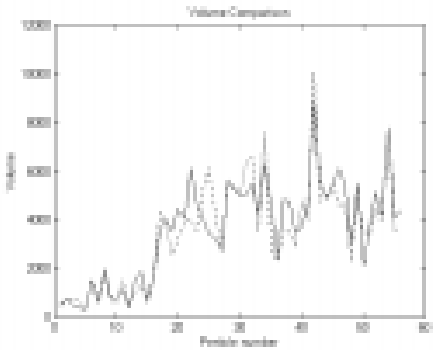

(h) particle \#386 \#440

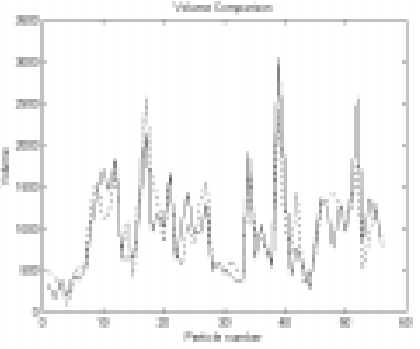

(f) particle \#276 \#330

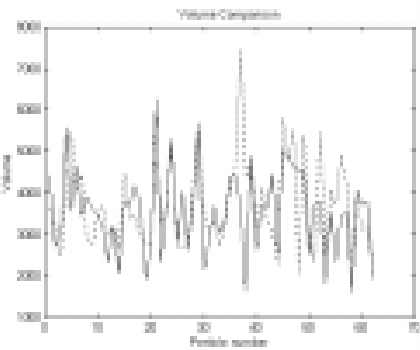

(i) particle \#441 \#501

Figure 6.7 Modeling Effect Observation

\subsection{Model Adequacy}

To simplify the statistical analysis of the model, Eqn. (6.19) is treated as an approximated standard MLR model with zero intercept. A number of techniques can be used to measure the adequacy of a multiple regressor model [16]. Two of those techniques to present the adequacy of the model were used. The pertinent theoretical basis can be reviewed in the book by Hines and Montgomery [16] and Myens [18].

\subsubsection{The Coefficient of Multiple Determination}

The coefficient of multiple determination, in a loose sense, is a measure of the amount of reduction in the variability of the response obtained by using the regressor 
variables [16], is defined in the problem as

$$
R^{2}=1-\frac{S S_{E}}{S_{v v}}
$$

where

$$
\begin{gathered}
S S_{E}=\mathbf{a}^{T} \mathbf{a}=\sum_{i=1}^{n=501}\left(\hat{V}_{i}-V_{i}\right)^{2} \\
S_{v v}=\sum_{i=1}^{n=501}\left(V i-\bar{V}_{i}\right)^{2}=\mathbf{V}^{T} \mathbf{V}-\frac{\left(\sum_{i=1}^{n=501} V_{i}\right)^{2}}{n}
\end{gathered}
$$

where $S S_{E}$ is the sum of squares for error, and $S_{v v}$ is the sum of squares.

For the modeling sample of 501 particles, it was calculated that $S S_{E}=118075237$, $S_{v v}=1257933386$, thus $R^{2}=0.91$. This indicates that about $91 \%$ of the variability in the actual volume $V$ has been explained when the three regressor variables are used in the model.

\subsubsection{Error Normality}

One of the assumptions for fitting a regression model is that the errors are uncorrelated random variables and normally distributed with mean zero and constant variance, i.e., $\operatorname{NID}\left(0, \sigma_{e}^{2}\right)$. Fig. 6.8 shows the errors plotted against the particle sequence. Note that it follows the same particle sequence as that used in Fig. 6.6. 


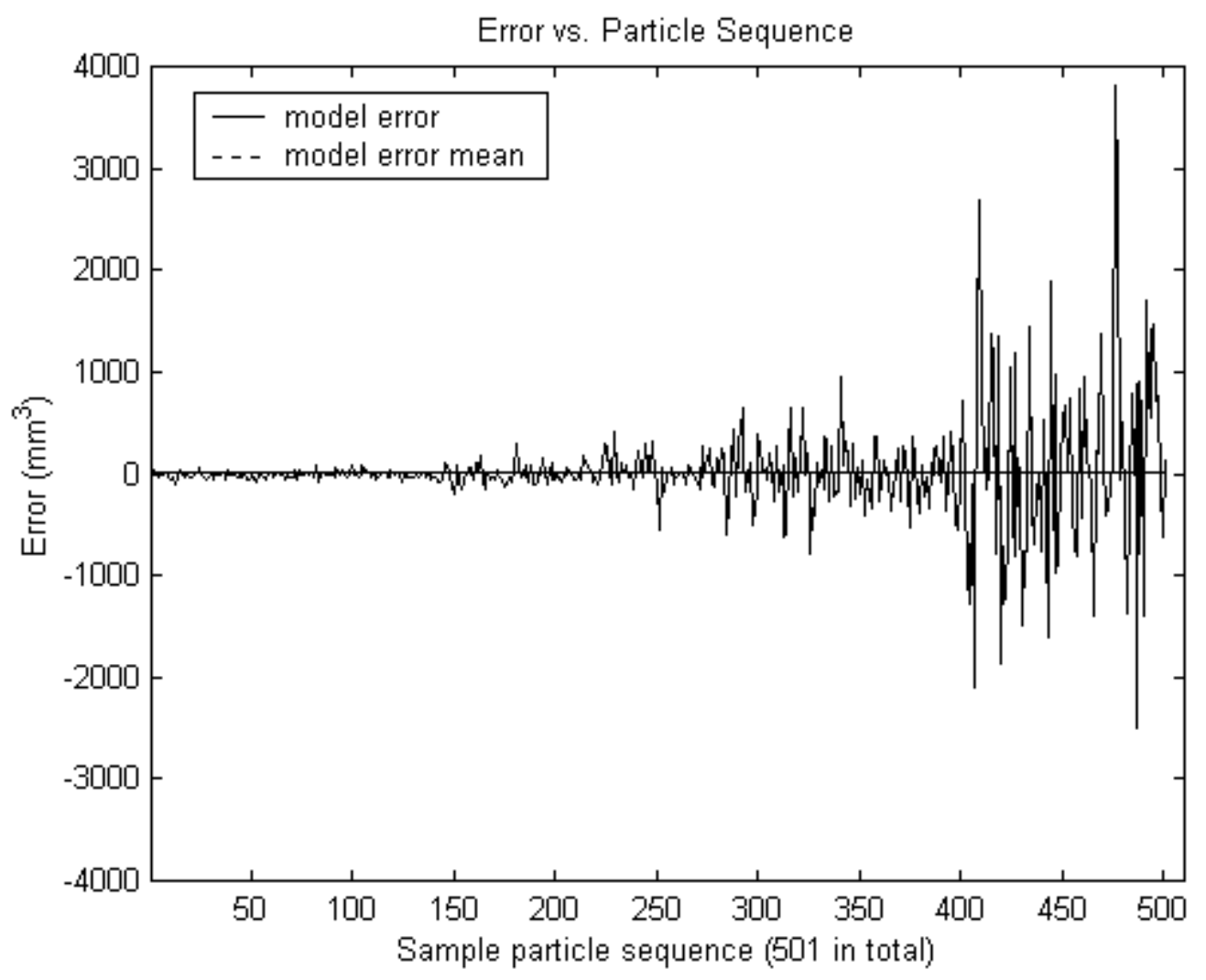

Figure 6.8 Model Error vs. Particle Sequence

To test the normality of the residual which is the difference between actual volume and estimated volume, one may standardize the residuals by computing the quantity

$$
d_{i}=\frac{\varepsilon_{i}}{\sqrt{M S_{E}}}
$$

where $i=1,2,3, \ldots, \mathrm{n}$

$M S_{E}:$ mean squares, and

$$
E\left(M S_{E}\right)=\sigma_{e}^{2}
$$

where $E(\bullet)$ denotes the expectation, and $\sigma_{e}^{2}$ is the error variance [16]. 
If the error are $\operatorname{NID}\left(0, \sigma_{e}^{2}\right)$, then approximately $95 \%$ of the standardized residuals should fall in the interval $(-2,2)$. Fig. 6.9 presents the histogram of the $d_{I}$ 's for the sample.

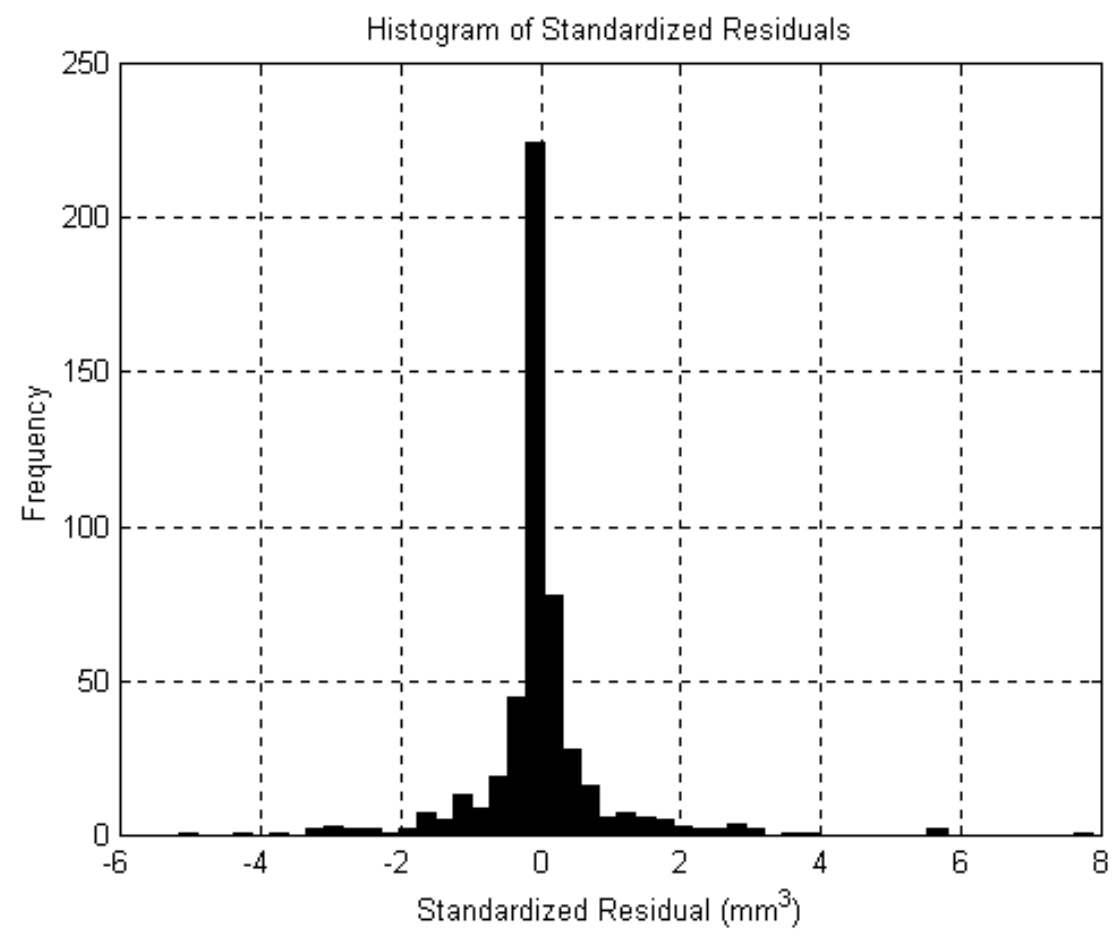

Figure 6.9 Histogram of Standardized Residuals

The percentage of the resultant standardized residuals that are within $(-2,2)$ is found to be $94.01 \%$, the errors can thus be regarded as being distributed normally.

\subsection{Model Testing}

The quality of the developed volumetric model needs to be tested using the randomly selected samples. If the sample population satisfies the size range (minor diameter is between $4.75 \mathrm{~mm}$ and $25.00 \mathrm{~mm}$ ), it is reasonable to expect a satisfactorily accurate result. 


\subsubsection{Preparation of Testing Sample Population}

Due mainly to the size constraints of the lighting box on which the particles are placed, the number of particles in an image is limited. Eight batches of testing sample were prepared. Particles in each batch were then placed in the imaging scene and were photographed. The same particles were photographed five times. Each time the particles were "stirred up" to give a completely new image. The five images of each batch were marked with $a, b, c, d, e$ respectively for analysis, thus there were 40 total images in the sample.

To test the model, one out of five images from each sample batch was randomly selected. The statistics of the each batch are tabulated in Table 6.1. The histograms of some useful measurements for each and for the combined batch are included in Appendix II. The total actual mass of the each batch is obtained by weighing.

Table 6.1 Sample Measurement Statistics

\begin{tabular}{|c|c|c|c|c|c|c|c|c|}
\hline $\begin{array}{c}\text { Batch / } \\
\text { Image } \\
\#\end{array}$ & $\begin{array}{c}\text { Total } \\
\text { particle } \\
\#\end{array}$ & $\begin{array}{c}\text { Total } \\
\text { true } \\
\text { mass }(g)\end{array}$ & $\begin{array}{l}\text { Area } \\
\text { mean } \\
\left(\mathrm{mm}^{2}\right)\end{array}$ & $\begin{array}{l}\text { Area } \\
\mathrm{STD}^{+} \\
\left(\mathrm{mm}^{2}\right)\end{array}$ & $\begin{array}{c}\text { Major } \\
\text { diameter } \\
\text { mean } \\
(\mathrm{mm})\end{array}$ & $\begin{array}{c}\text { Major } \\
\text { Diameter } \\
\mathrm{STD}^{+} \\
(\mathrm{mm})\end{array}$ & $\begin{array}{c}\text { Minor } \\
\text { diameter } \\
\text { mean } \\
(\mathrm{mm})\end{array}$ & $\begin{array}{c}\text { Minor } \\
\text { Diameter } \\
\mathrm{STD}^{+} \\
(\mathrm{mm})\end{array}$ \\
\hline$\# 1 / b$ & 345 & 316.25 & 96.3661 & 40.0473 & 11.4350 & 3.0401 & 7.8602 & 2.2699 \\
\hline \#2 /a & 376 & 265.91 & 75.2003 & 38.4560 & 9.8945 & 3.4555 & 6.5525 & 2.1892 \\
\hline$\# 3 / \mathrm{e}$ & 215 & 475.06 & 169.2037 & 81.5658 & 15.4329 & 4.7307 & 10.8713 & 3.6119 \\
\hline$\# 4 / d$ & 251 & 383.46 & 125.3001 & 75.1036 & 13.1246 & 4.4820 & 8.8168 & 3.6209 \\
\hline$\# 5 / a$ & 378 & 625.52 & 128.0232 & 78.6642 & 13.1727 & 5.0281 & 8.8973 & 5.9956 \\
\hline \#6 / a & 76 & 512.02 & 362.9582 & 197.1795 & 23.5464 & 7.7220 & 16.1730 & 5.3785 \\
\hline$\# 7$ / c & 71 & 521.38 & 376.1573 & 204.8211 & 23.1580 & 7.3076 & 17.3936 & 6.2691 \\
\hline$\# 8 / \mathrm{e}$ & 150 & 1006.19 & 356.2143 & 206.9573 & 22.5798 & 8.0629 & 16.2522 & 6.1154 \\
\hline
\end{tabular}

$\mathrm{STD}^{+}:$Standard deviation. 


\subsubsection{Testing Results}

For five images from the same batch, eight batches in total, using the volumetric model and the same density used before to compute the estimated mass, sub-figure (a) shows the estimated mass of each image from batch \#1 to \#8. So, for a single batch, every five mass estimates are plotted versus one actual mass. In (b), for each batch, averaging these five estimated mass values as a data point, then plot them against the actual mass. (c) demonstrates the percent error from (b).

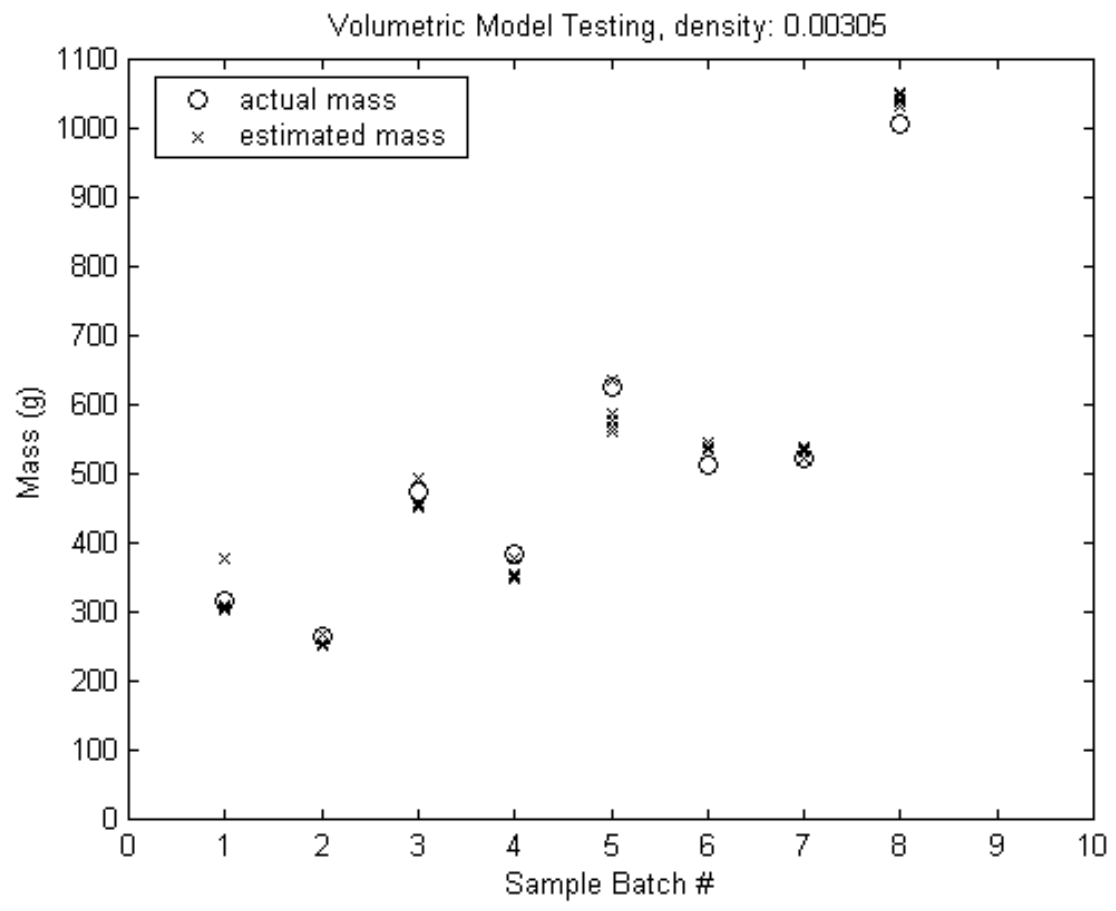

(a) mass comparison 


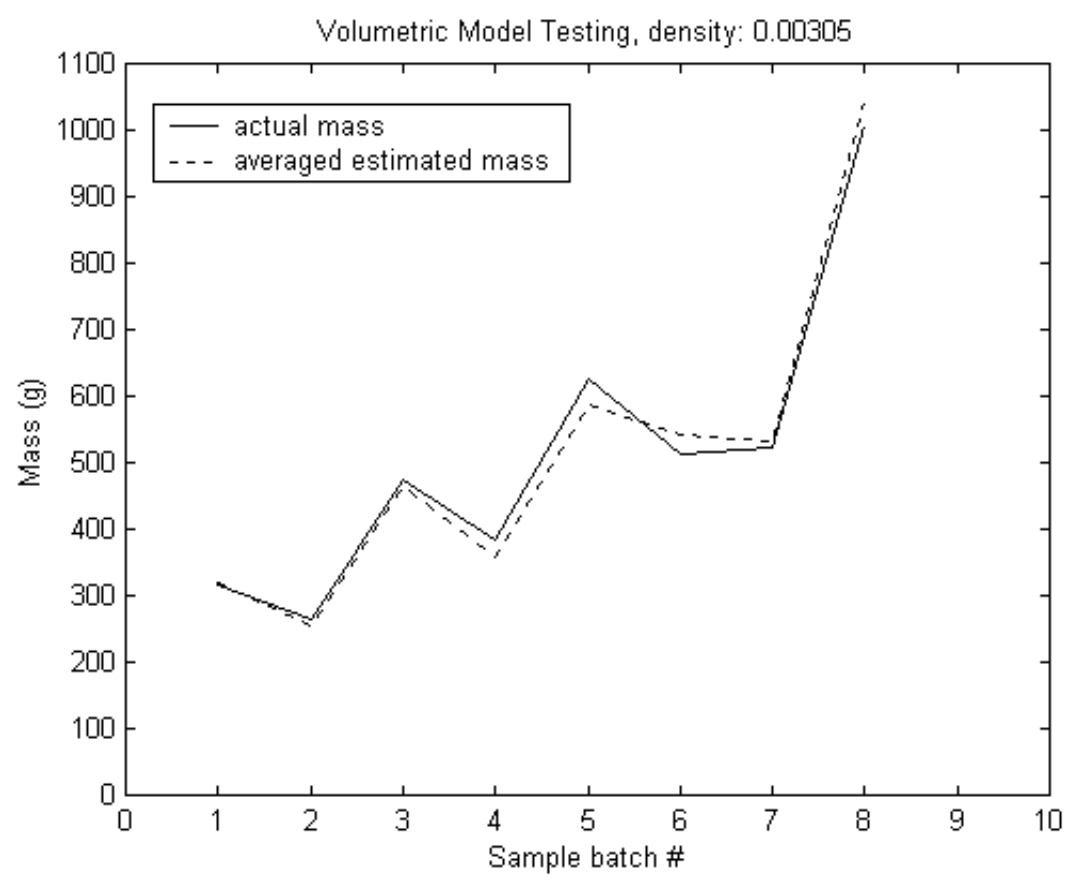

(b) mass comparison

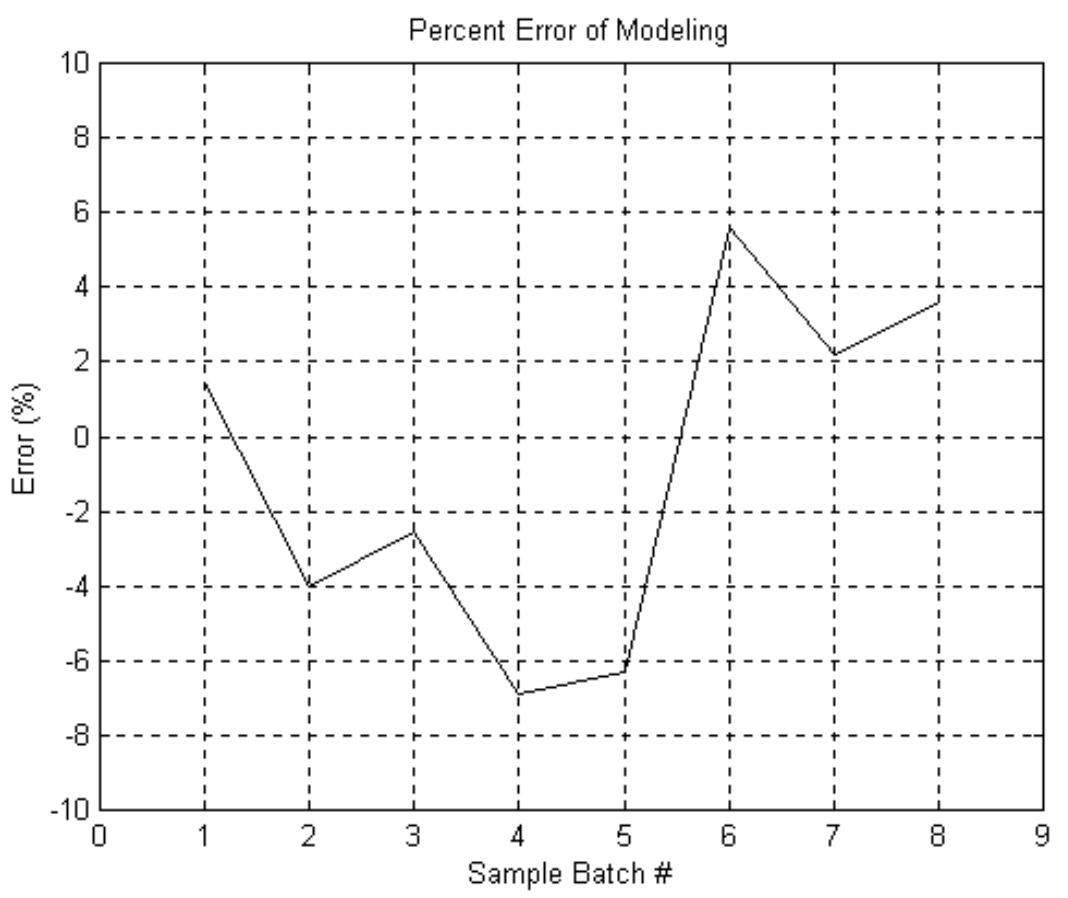

(c) percent error

Figure 6.10 Model Testing for Mass 
From sub-figure (c), it is observed that the percent error can reach as high as

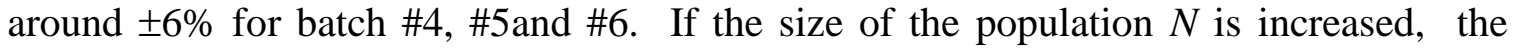
accuracy can then be improved. To do this, take a random combination of the images, and each image of the combination was randomly selected from one of the eight batches. This indicates that the total number of particles will increase up to $N=1862$, which is a total particle number of eight batches. Fig. 6.11 illustrates such a random combination formed by image $b, a, d, e, c, e, c, d$ from the sample batch $\# 1, \# 2, \ldots \# 8$, respectively. 


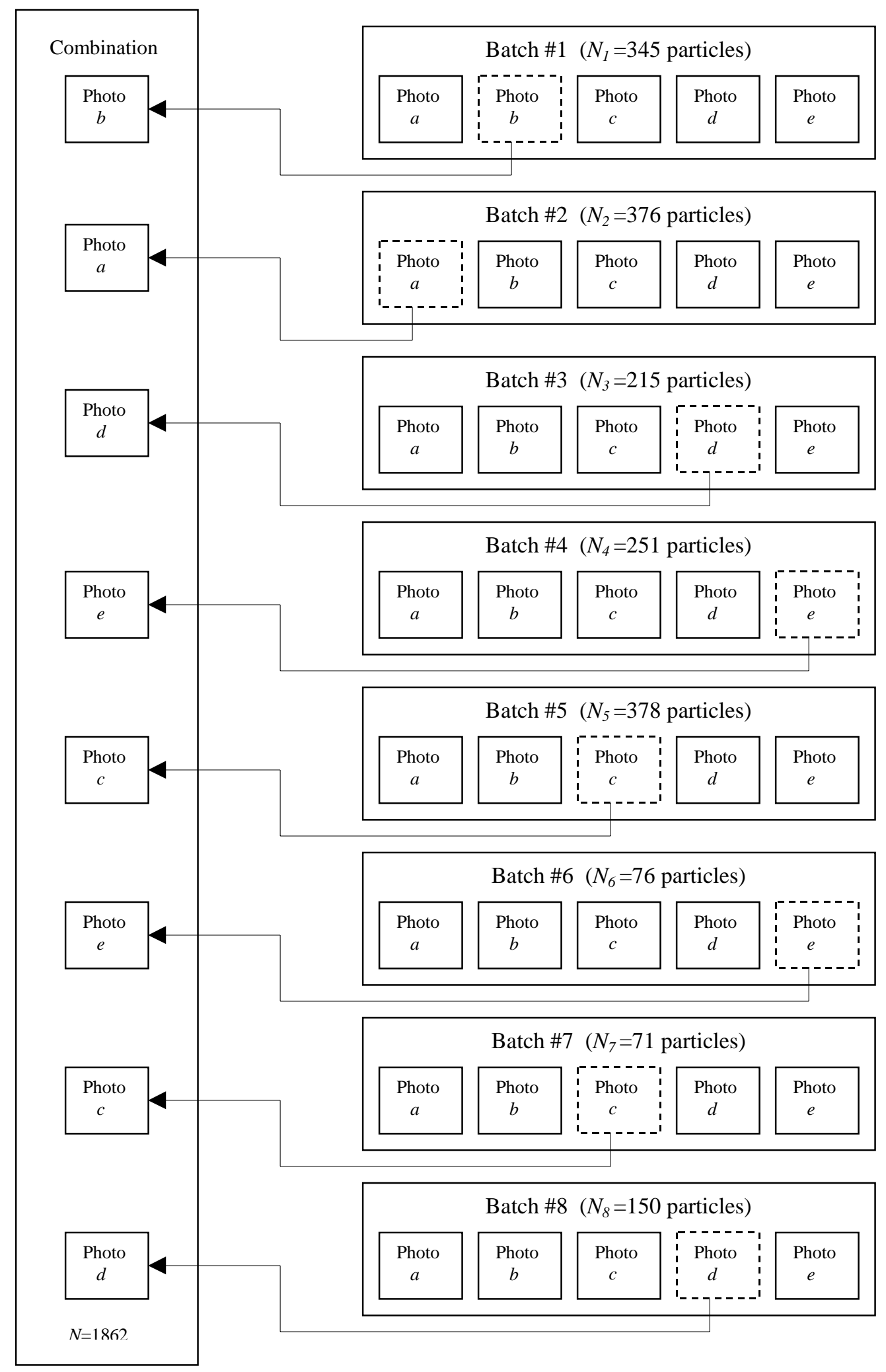

Figure 6.11 A Random Combination of Sample Batches (badececd) 
100 combinations were tested. Each combination may represent one "grand" image covering all 1862 particles. Each new constituent image results from a new "stirup", and in total 100 stir-ups were conducted for producing 100 grand images. The result is shown in Fig. 6.12. Fig. 6.13 shows the percent error, which mainly stays within $\pm 2 \%$, and is greatly reduced as anticipated.

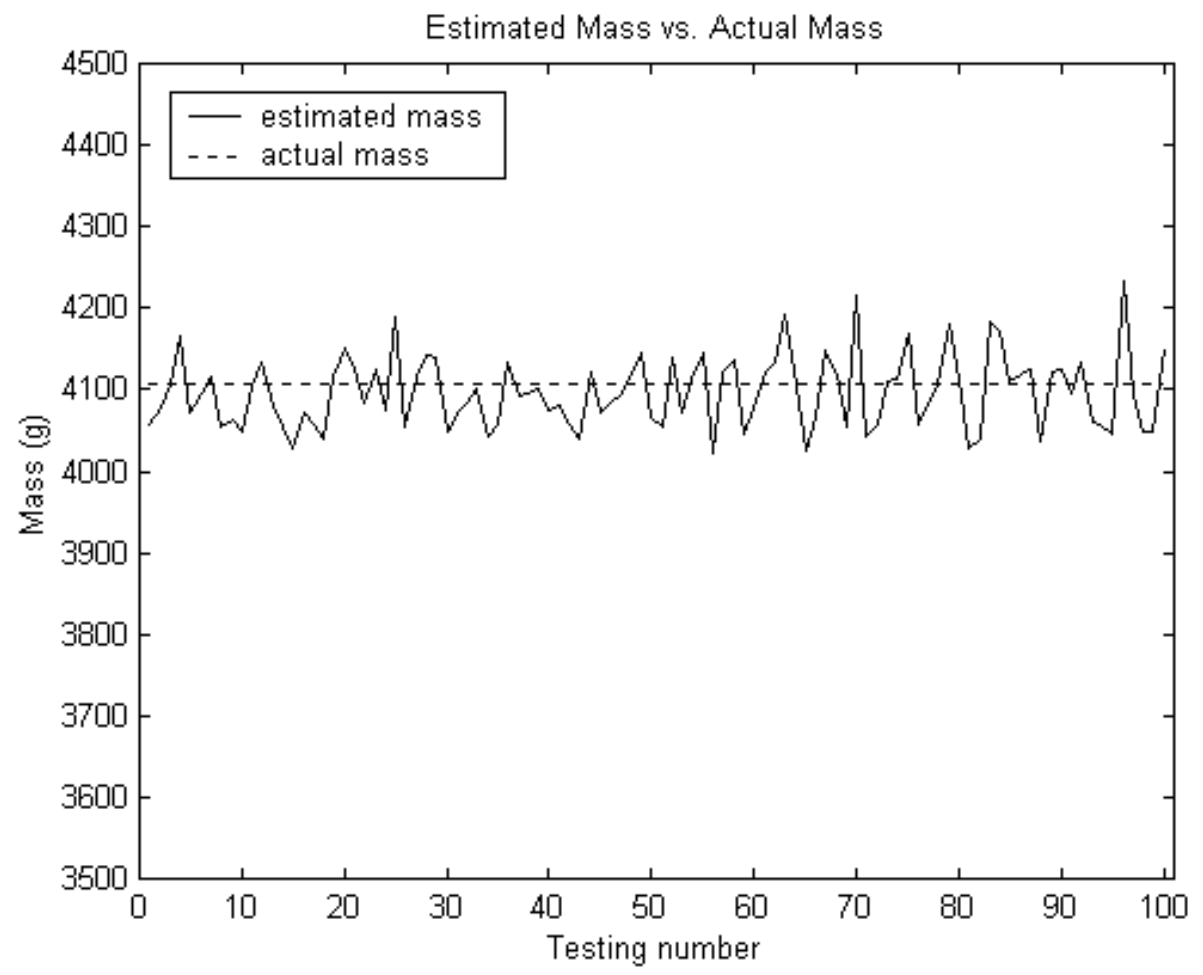

Figure 6.12 Modeling Result for 100 Combinations 


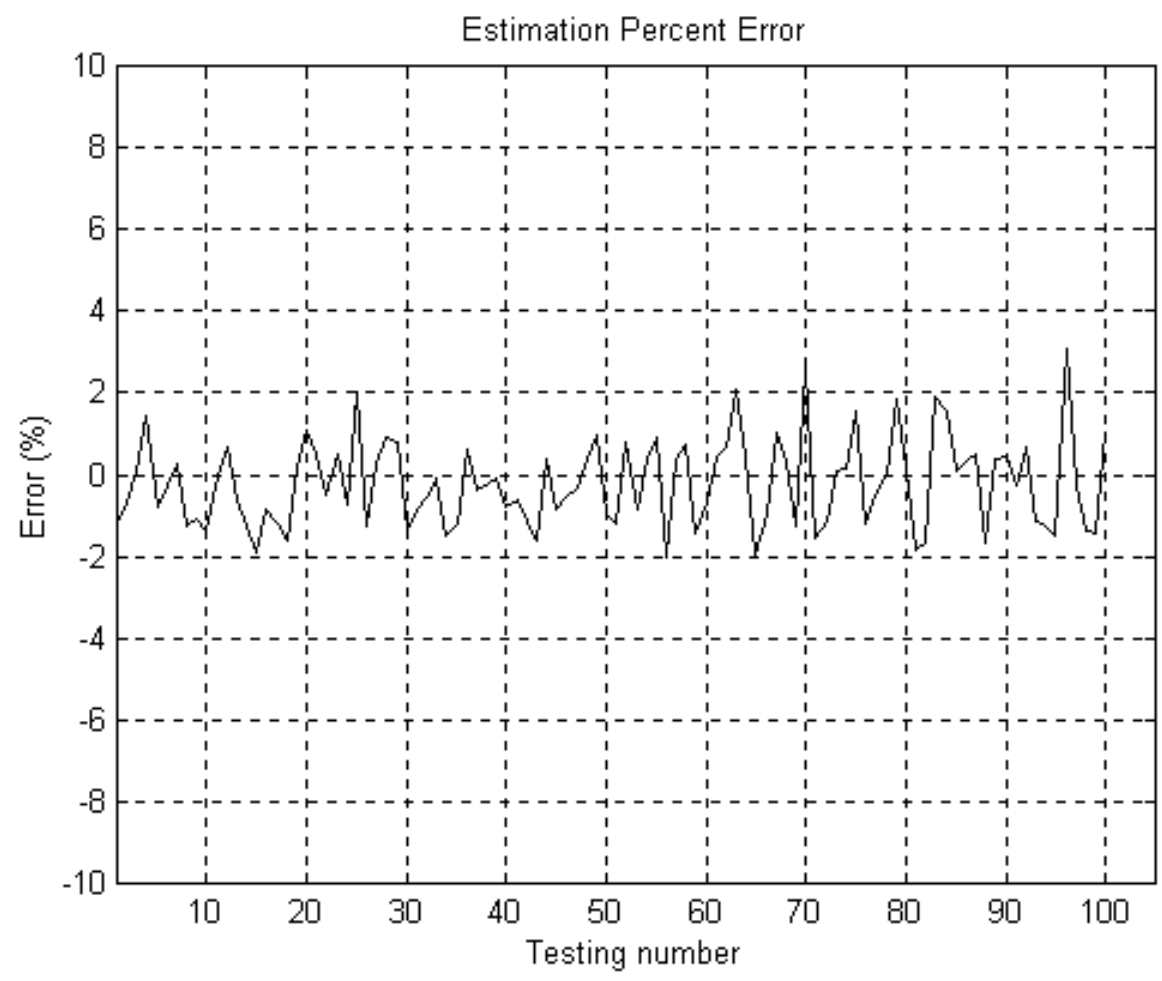

Figure 6.13 Percent Error for 100 Combinations 


\section{SIEVING CORRELATION}

\subsection{Introduction}

Since the Superpave specifications are given on the basis of percent passing by mass, two correlations must be made. First, particles are optically sieved into "bins" or sizes based on the minor dimension of the particle. In each bin, the number of particles is not important, rather, the total mass of the particles in that bin is. Second, particle's mechanical sieving behavor must be correlated with the optical sieving behavor. The model which correlates information obtained from the 2-D image to the volume has been established. In this chapter, a sieving correlation method was developed by first modeling the particle cross section into a rectangle, plus the scaling factors obtained from calibration to take the actual cross section shape and other related elements into account. Based on the flatness distribution from 501 sample particles, a criterion was set to determine the sieving strategy for each particle. Results are presented as gradation curves in the format used for Superpave analysis. The optical sieving results were also compared against mechanically sieved measurements of the same samples to test accuracy. The results showing satisfactory correspondance were presented.

\subsection{Identified Sieving Problems}

Either a circular sieve opening or a cylindrical particle as shown in Fig. 7.1 (a) will make the sieving process easy to describe analytically. In either case, the fashion in which the particles fall through the sieve opening is rotation invariant in the falling direction. Unfortunately, neither the sieve opening shape nor the cross section of the particle is circular. Sieve openings are square, and the cross section of crushed particles 
is randomly shaped as illustrated in Fig. 7.1 (b). This makes the sieving analysis rather complex.

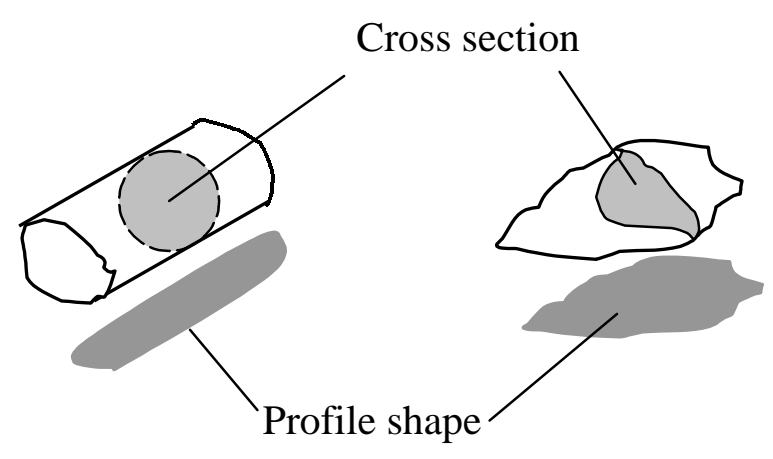

$\begin{array}{ll}\text { (a) circular cross section } & \text { (b) random shape cross section }\end{array}$

Figure 7.1 Particle Cross Section Shape

For example, for a fairly flat particle with $22 \mathrm{~mm}$ minor diameter, it is clear that it can not pass if its minor diameter is parallel to the square sieve opening of $19.00 \mathrm{~mm}$. However, after rotating a certain angle $\alpha$, it becomes passable since $22 \mathrm{~mm}$ is smaller than the diagonal $(26.87 \mathrm{~mm})$ of the square opening, as shown in Fig.7.2. There is a tendency for flat particles, especially those with rounded edges to pass diagonally through the sieve. 


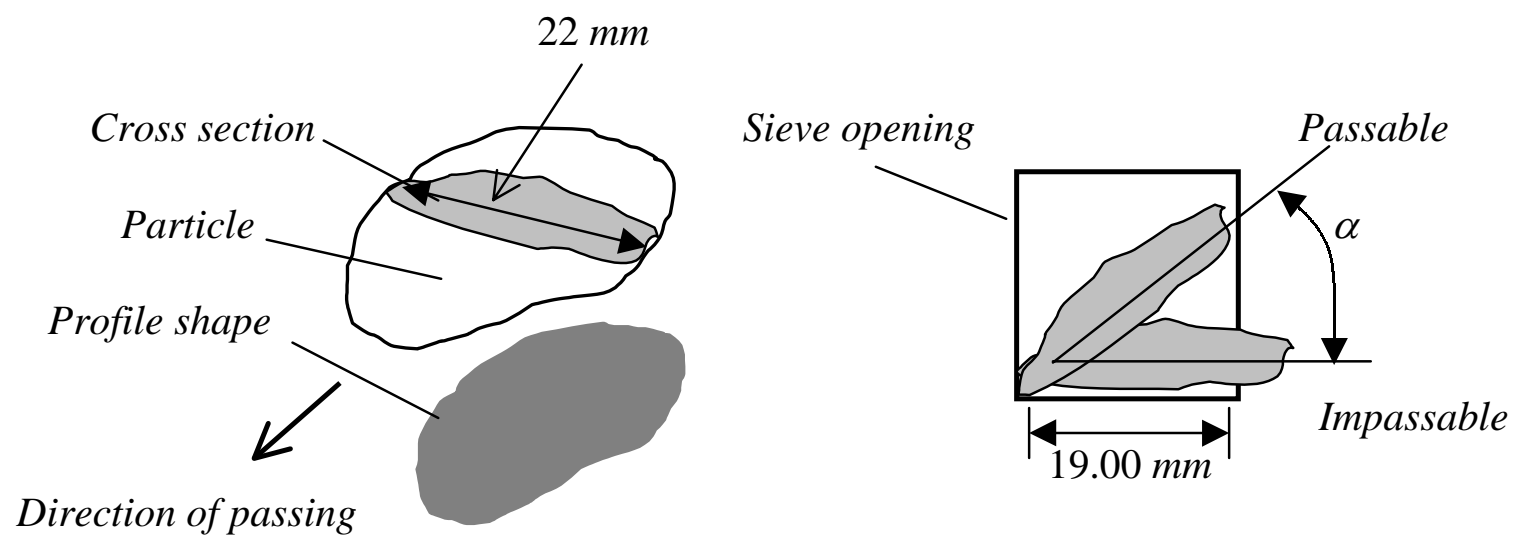

Figure 7.2 Particle Passing Mechanism

In vision sieving, the particle minor diameter is approximately known, but particle thickness is not, and the thickness is undoubtedly a critical factor that influences particle sieving behavior. This requires development a method for relating the 2-D observable features to the sieving behavior of particles. It will probably be impossible to do this with extreme accuracy for individual particles. However, it is expected that reasonably accurate results for bulk measurements should be obtained.

\subsection{Analytical Discussion}

To simplify the complexity of the sieving behavior problem, the interaction between the particle cross section and the square mesh of a given sieve was first analytically modeled. Let $d_{s v}$ be the size of a given sieve, and as defined previously, $D_{\text {min }}$ be the minor diameter, and $H_{\text {nom }}$ be the nominal height. This way the cross section takes the shape of a rectangle, as illustrated in Fig. 7.3. 


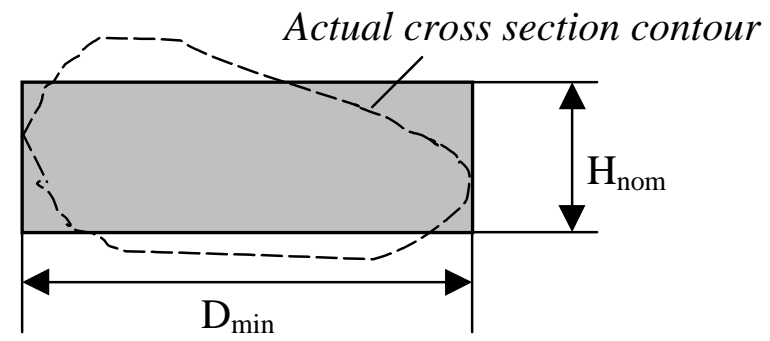

\section{Figure 7.3 Modeled Particle Cross Section}

The interaction of the cross section with the sieve size takes place only for those

particles whose minor diameters are within the range $d_{s v}<D_{\min }<\sqrt{2} d_{s v}$. The reason that the equal sign "=" was not included is that, if particle minor diameter equals the sieve size, it is expected to pass, while if it has the same dimension as the diagonal of square mesh, it will be retained with certainty, since particles are not "razor thin".

Given a sieve mesh size $d_{s v}$, the critical position for a particle with assumed rectangular cross section of minor diameter $D_{\min }$ and nominal height $H_{\text {nom }}$ to pass or be retained in the sieve is illustrated in Fig. 7.4. 


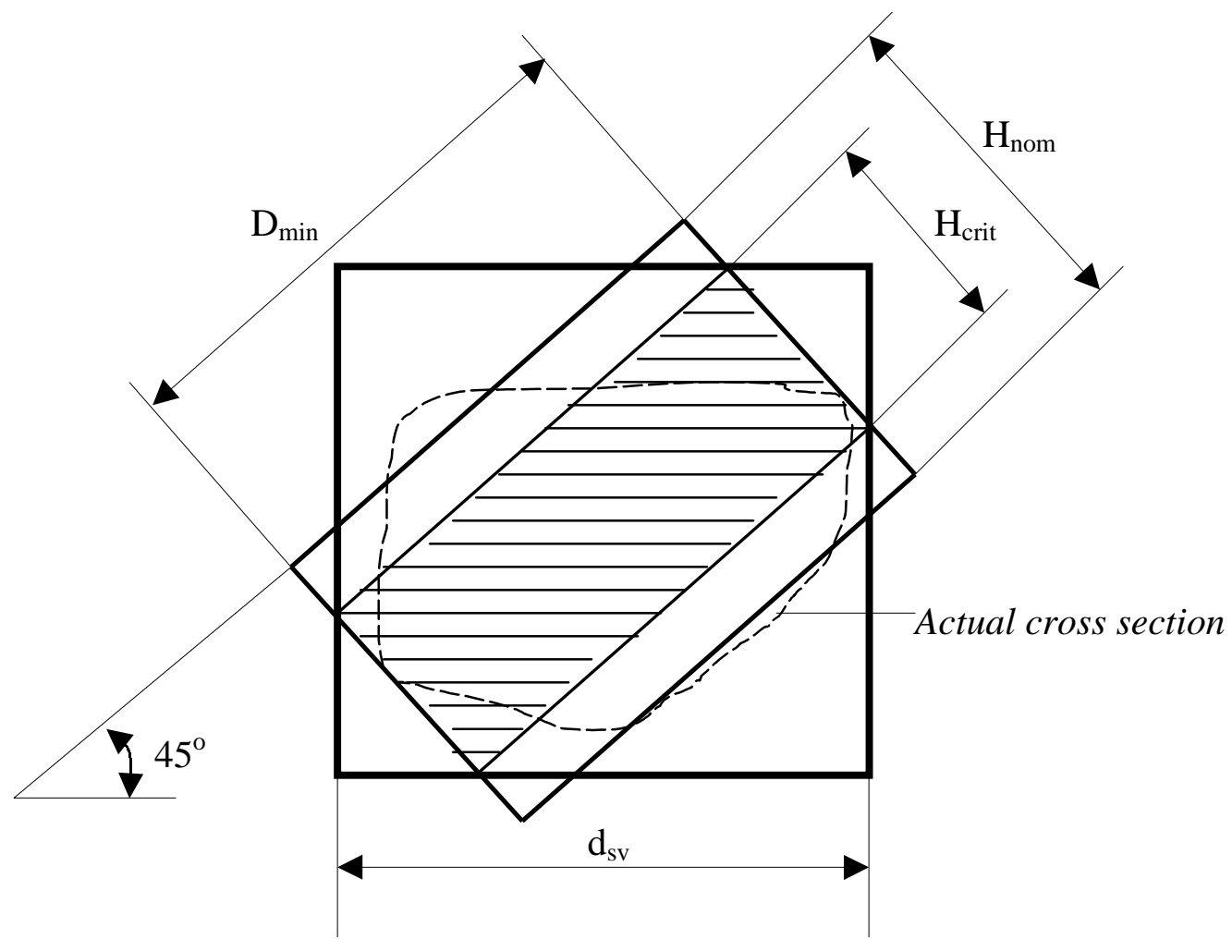

Figure 7.4 Critical Position

For any given minor diameter $D_{\min }$ satisfying $d_{s v}<D_{\min }<\sqrt{2} d_{s v}$., it has a "critical height", denoted as $H_{c r i t}$ in above figure. The hatched rectangle is correspondingly formed by $D_{\min }$ and $H_{c r i t}$, as also shown in above figure. If the nominal height $H_{n o m}$ is shorter than $H_{c r i t}$, the particle passes this sieve, otherwise it is retained. The following simple relation holds for $H_{c r i t}$ and $D_{\min }$ :

$$
H_{c r i t}=\sqrt{2} d_{s v}-D_{\min }
$$


Two special cases determine the range of $H_{\text {crit }}$

i) if $D_{\text {min }}=\sqrt{2} d_{s v}$, then $H_{c r i t}=0$. This means that unless the particle is "razor thin", it will be retained on the sieve. Particles with this $D_{\min }$ will always be retained in practice.

ii) if $D_{\min }=d_{s v}$, then $H_{c r i t}=0.4142 D_{\min }$. Particles with this $D_{\min }$ will be expected to pass the sieve.

From i) and ii), the range of the critical height is thus

$$
0<H_{c r i t}<0.4142 D_{\min }
$$

Recall that the flatness of the particle is defined as

$$
Y_{f l a t}=\frac{H_{n o m}}{D_{\min }}
$$

and further define the critical flatness for a particle of $D_{\min }$ as

$$
Y_{\text {crit }}=\frac{H_{c r i t}}{D_{\min }}
$$

Apparently, the state of any particle of $D_{\min }$ in the sieve can be determined by the following conditions:

$$
\left\{\begin{array}{c}
\text { if }: Y_{\text {flat }} \leq Y_{\text {crit }}, \text { pass } \\
\text { if }: Y_{\text {flat }}>Y_{\text {crit }}, \text { retained }
\end{array}\right.
$$

One may argue that for the crushed limestone aggregates, the shapes of the cross section are not retangular, so the conditions set in inequality (7.4) are not sufficient to determine a particle's passing or being retained. To remedy this, a calibration factor is obtained experimentally and added to correct the above conditions, as described later . 


\subsection{Sieving Probability Analysis}

For any particle with $D_{\min }$ in a sieve, the conditions expressed in inequality (7.5) are fundamental for judging its ultimate state: passing or being retained. From Eqn. (7.1) and (7.4), the corresponding critical flatness can thus be easily obtained as

$$
Y_{c r i t}=\sqrt{2} \frac{d_{s v}}{D_{\text {min }}}-1
$$

Now the question arises: what is the flatness, $Y_{\text {flat }}$, of the particle of interest?

The sample of 501 particles was analyzed to determine the probability distribution of the particle flatness. Fig. 7.5 and Fig. 7.6 present a scatter diagram and a histogram of the sample flatness statistics.

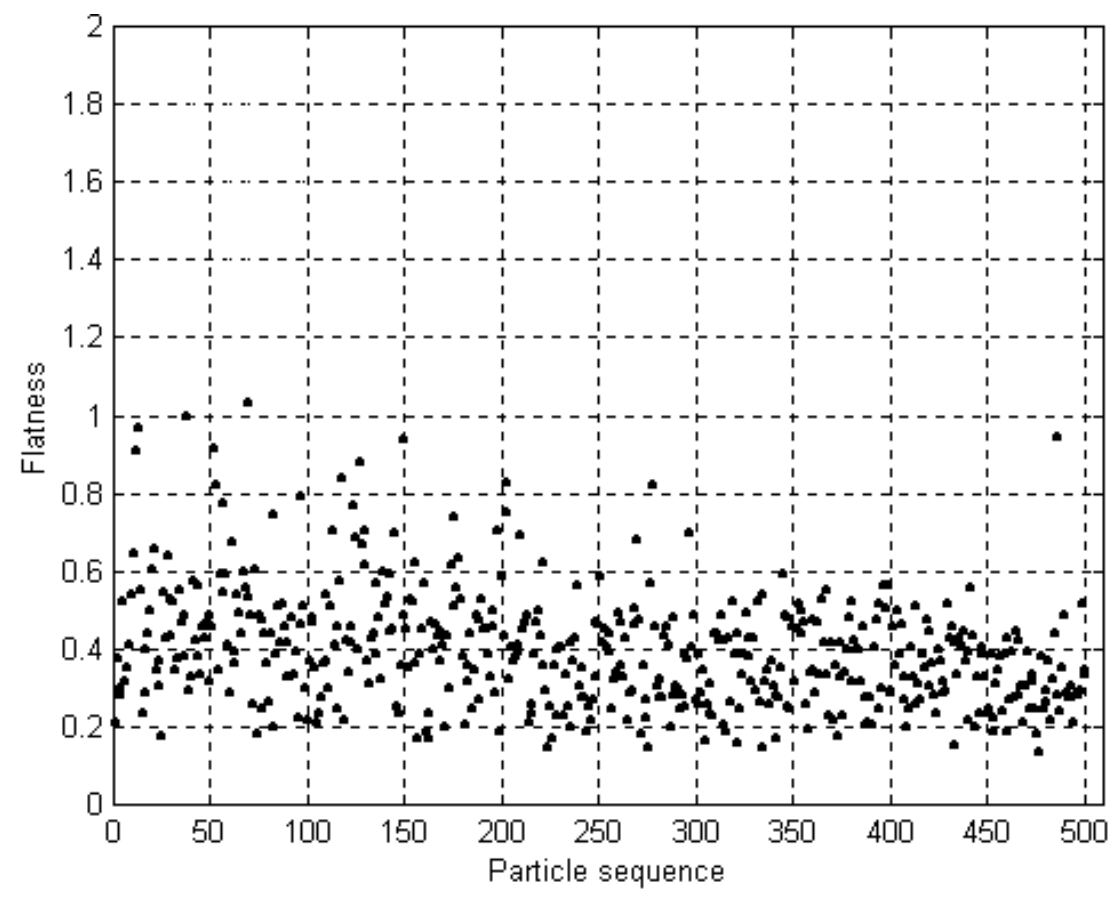

Figure 7.5 Scatter Diagram of Sample Flatness 


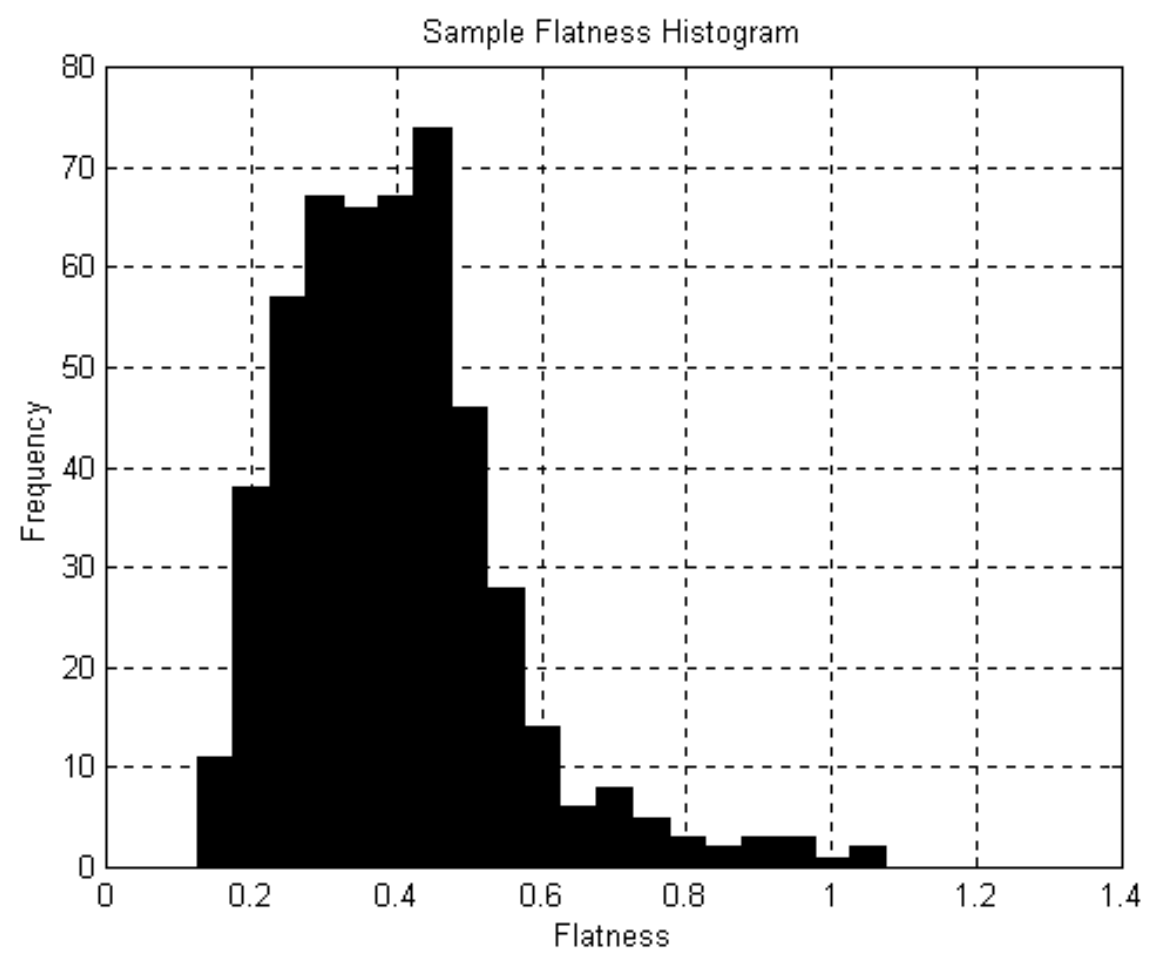

Figure 7.6 Sample Flatness Histogram

The cumulative distribution of the 501 sample particles is obtained from the probability density data. Its approximated distribution function is attained by applying a curve-fitting technique, shows as the solid curve in Fig. 7.7. The distribution function is approximated as

$$
P\left(Y_{c r i t}\right)=-19.9524 Y_{c r i t}^{5}+67.4 .1075 Y_{c r i t}^{4}-83.6898 Y_{c r i t}^{3}+44.4792 Y_{c r i t}^{2}-7.6510 Y_{c r i t}+0.4069
$$




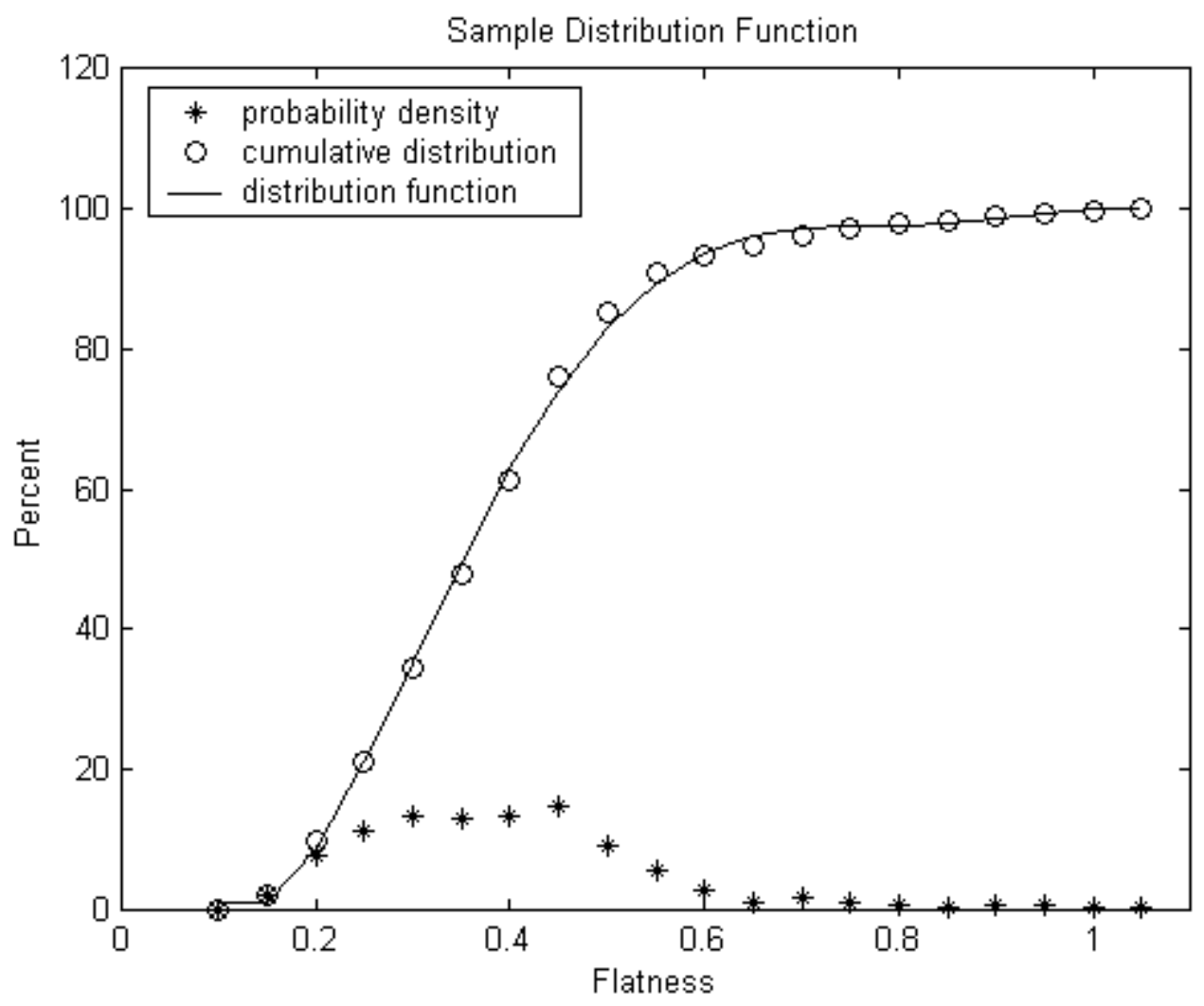

Figure 7.7 Sample Flatness Distribution

The strategy for using the function in Eqn. (7.7) is straightforward:

1) Given the particle's measured minor diameter $D_{\min }$, select those particles for which $d_{s v}<D_{\min }<\sqrt{2} d_{s v}$.

2) Calculate the $H_{\text {crit }}$.

3) From the cumulative probability distribution function in Eqn. (7.7), calculate

$$
P^{*}=\left\{P\left(Y_{\text {flat }}\right) \leq Y_{\text {crit }}\right\}
$$

where $P(\bullet)$ indicates probability.

4) Create a random number $\eta$ on $[0,1]$ using a uniform distribution.

5) If $\eta \leq P^{*}$, the particle passes. Otherwise it is retained. 
This approach makes no attempt to determine analytically whether an individual particle passes or is retained. Rather, it exploits the central tendency of the data to provide an estimate which proves to be fairly accurate over a large sample.

For the sake of convenience, rewrite inequality (7.5) which are the conditions for sieving behavior.

$$
\left\{\begin{array}{c}
\text { if }: Y_{\text {flat }} \leq Y_{\text {crit }}, \text { pass } \\
\text { if }: Y_{\text {flat }}>Y_{\text {crit }}, \text { retained }
\end{array}\right.
$$

Recall that inequalities expressed in (7.9) are based on the assumption that the particle has rectangle shaped cross section which is not true in reality. Also, the conditions in (7.9) is for analytical determination whether an individual particle passes or is retained. At this point, the analytical determination for an individual partilcal has been converted to statistical determination for a group of particles. This fact imposes an additional modification to $P^{*}$ to compensate for inaccuracies caused by irregular shaped cross section. Referring to the steps in using sample flatness distribution function stated previously, multiply $P^{*}$ by a scaling factor called sieving calibration factor, $\zeta_{s v}$, where $\zeta_{s v} \geq 1.00$. Then conditions for sieving behavior becomes

$$
\left\{\begin{array}{c}
\text { if }: \eta \leq \zeta_{s v} P^{*}, \text { pass } \\
\text { if }: \eta>\zeta_{s v} P^{*}, \text { retained }
\end{array}\right.
$$

where $\zeta_{s v}$ can be calibrated experimentally for the given sieve size.

\subsection{Sieving Phase Analysis}

\subsubsection{Size Modification for Triangular Shapes}

The minor diameter of the shape is regarded as the size that determines the sieving behavior. For triangular shaped particles as shown in Fig. 7.8, however, the 
actual sieve size will be greater than the minor diameter, to an extent that may vary according to the actual shape. This requires to sort out those triangle shapes, and modify the minor diameter that is the most vital element to determine passing or staying on a given sieve.

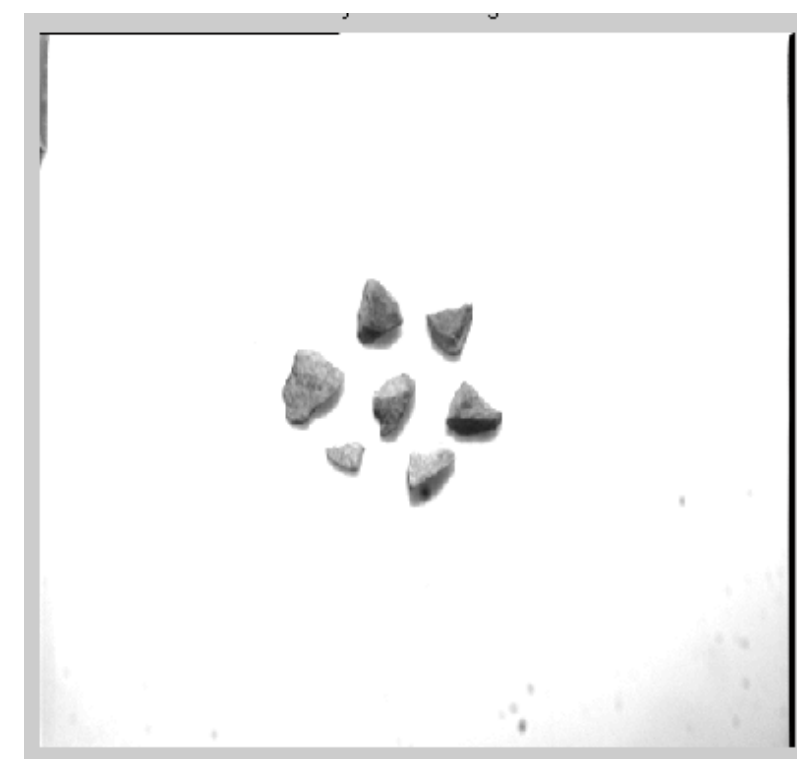

Figure 7.8 Triangle Shaped Particles

Rather than determine the modifying extent for each triangular particle, the minor diameter is multiplied by a correction factor, which can be obtained experimentally. This factor found to work well is:

$$
D_{\text {min }}^{*}=D_{\text {min }}(1+\mu)
$$

where

$D_{\text {min }}^{*}$ : modified minor diameter.

$\mu:$ a uniformly distributed random number within $[0,0.3]$. 


\subsubsection{Sieve Series and Sieving Phase}

The same sample population that had been applied to test the volumetric model was used to determine the calibration factors, $\zeta_{s v}$. As stated previously, it consists of eight batches, whose basic statistics are tabulated in Table 6.1. The histograms of some measurements for each batch and for the total sample are included in Appendix II.

The sieve set consists of five sizes: $4.75 \mathrm{~mm}, 9.50 \mathrm{~mm}, 12.50 \mathrm{~mm}, 19.00 \mathrm{~mm}$, and $25.00 \mathrm{~mm}$. They are stacked with the largest mesh size at the top, with successively smaller mesh sizes below, as shown in Fig. 7.9. For the sake of notational convenience, $d_{s v}$ denotes a sieve of any size. Note that the particles in investigation are poured in the top $25.00 \mathrm{~mm}$ sieve, falling through onto the next sieve below if not retained. Sieves are vibrated and rotated using an automatic sieve shaker. 


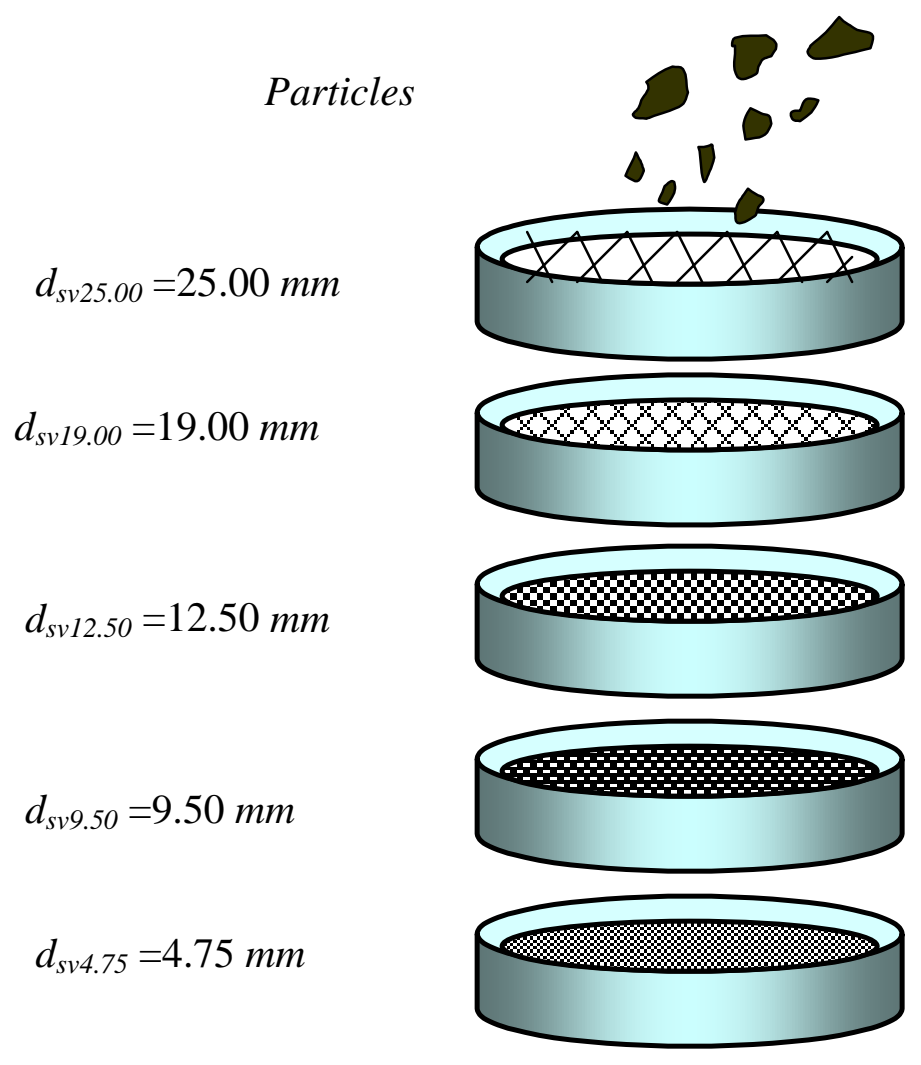

\section{Figure 7.9 Physical Sieve Cascade}

For a given sieve of size $d_{s v}$, only those particles whose minor diameter $D_{\text {min }}$, are

within the range $d_{s v}<D_{\min }<\sqrt{2} d_{s v}$, are candidates for sieving behavior consideration. The particles outside this range will be either retained in the upper sieve or pass through onto the lower sieve.

Fig. 7.10 outlines the overall sieving analysis for the whole prospective sample population. 
: A symbolic curve that means the particle may or may not be retained on the sieve.

1 : The state in which all particles within the corresponding size range are partially retained, i.e., some pass, some remain in the current sieve.

ए: The state in which all particles within the corresponding size range are absolutely retained.

: The state in which all particles within the corresponding size range are partially retained either in the sieve of $d_{s v 12.50}$, or in the sieve of $d_{s v 9.50}$.

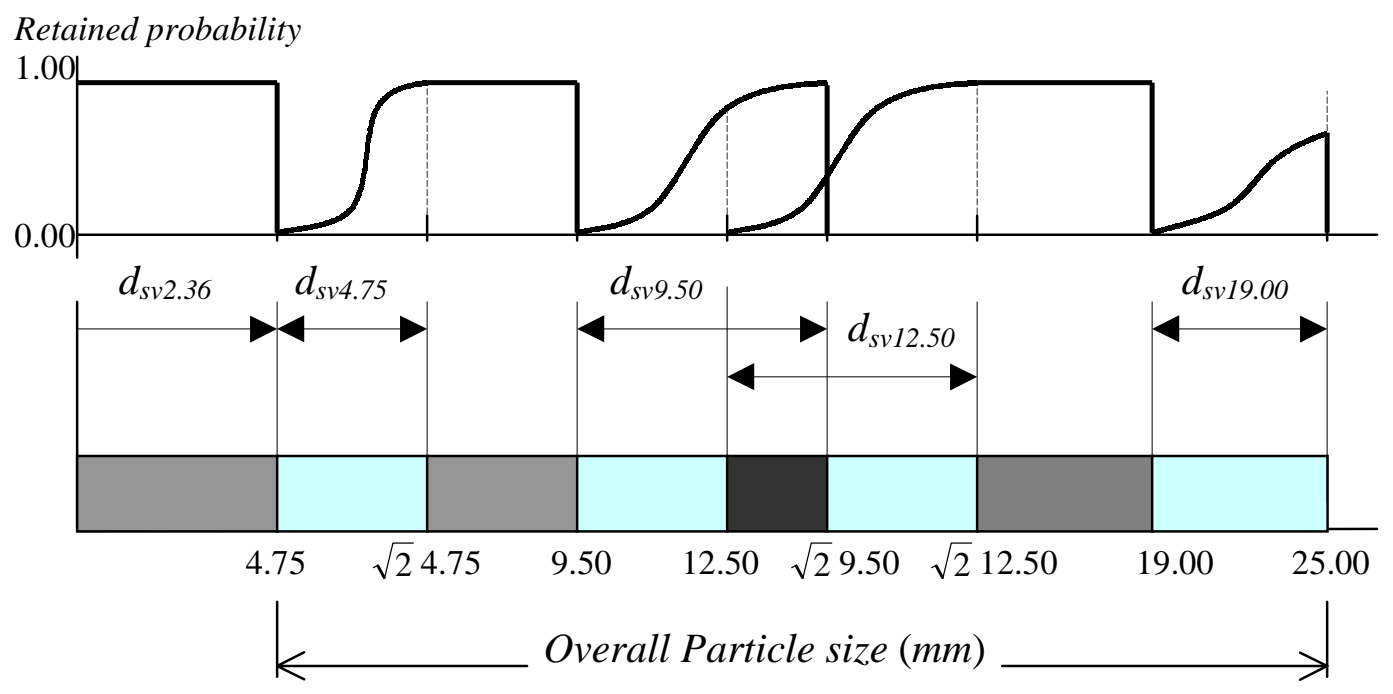

Figure 7.10 Overview of Sieving Phase Analysis

After all particles in the image scene have been processed, three available parameters can be used to conduct the vision sieving process: shape, minor diameter, and estimated mass, denoted as $S_{s h p}, D_{\min }$, and $\hat{M}$, respectively. For $N$ particles in the image 
scene having been processed, a data array can thus be formed ready for vision sieving processing:

$$
\left[\begin{array}{ccc}
S_{s h p, 1} & D_{\min , 1} & \hat{M}_{1} \\
S_{s h p, 2} & D_{\min , 2} & \hat{M}_{1} \\
\vdots & \vdots & \vdots \\
S_{s h p, N-1} & D_{\min , N-1} & \hat{M}_{N-1} \\
S_{s h p, N} & D_{\min , N} & \hat{M}_{N}
\end{array}\right]
$$

The shape parameter gives information about the triangle shaped particles, whose minor diameter must be modified to correctly relate them to their sieving behavior.

In the algorithm, the sieving starts off with scanning the first column in matrix (7.12), finding triangles and modifying their diameters. Then the second column is scanned to group particles by size into bins for the percent passing statistics. The elements in the third column are assigned to the corresponding "volume bins" (sieves) suitable to their minor diameters. At the end of the "binning" algorithm, the percent passing is computed as the Superpave specifies according to the formula:

$$
\frac{\sum_{k=1}^{m} \hat{M}_{k}}{\sum_{w=1}^{N} \hat{M}_{w}} \times 100
$$

where

$m:$ the total number of particles retained in the sieve in question.

$N$ : the total number of particles in the population. 
$\hat{M}_{k}:$ mass of $k^{\text {th }}$ particle in the sieve in question.

$\hat{M}_{w}:$ mass of $w^{\text {th }}$ particle in the population.

The pseudocode of the overall sieving procedure is given in Table 7.1. Note that the actual working code can be written in various ways, and the coding presented in the following pseudo code is not necessarily the optimal one. The major purpose here is to clearly present the logic thread imbedded in the optical sieving phase. 


\section{Table 7.1 Pseudocode of Sieving Procedure}

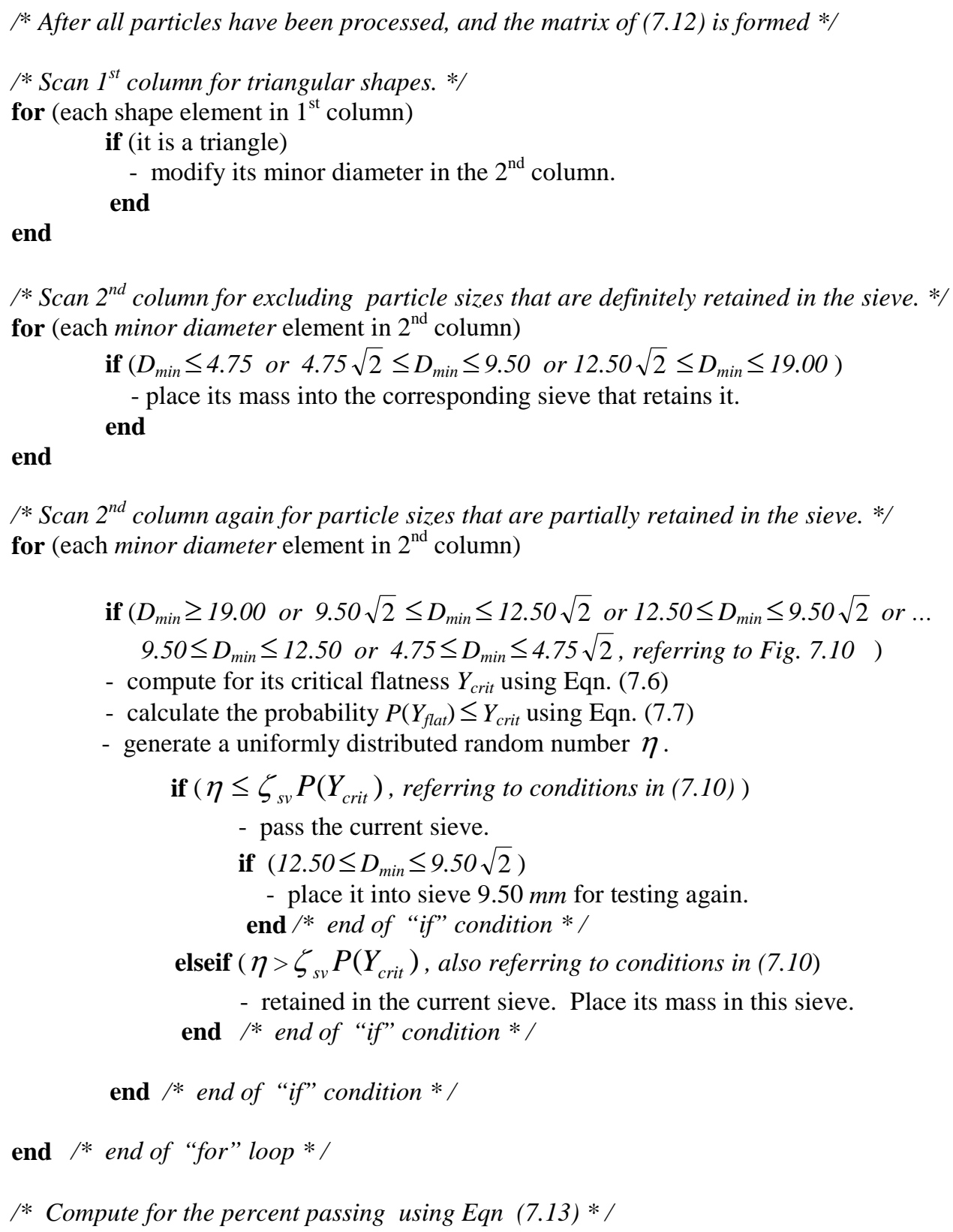

\subsection{Benchmark}

The particle size distribution (gradation) obtained from the vision sieving is calibrated against the results obtained from mechanically sieving the same sample. The 
result works as the "benchmark" for the sieving parameter calibration. The same eight batches of sample particles were used to obtain the benchmark.

The eight batches of sample particles are mechanically sieved. Each batch was sieved ten times in order to achieve better accuracy. As a result, for each batch, ten percent passing curves and ten percent retained curves were obtained. The desired results, the percent retained and percent passing, can be acquired. By percent retained, it means the percent of the particles in terms of volume (or mass) retained on each of the five sieves. As an example, Fig. 7.11 (a) and (b) shows the result for batch \#4 of percent retained and percent passing from mechanical sieving, respectively.

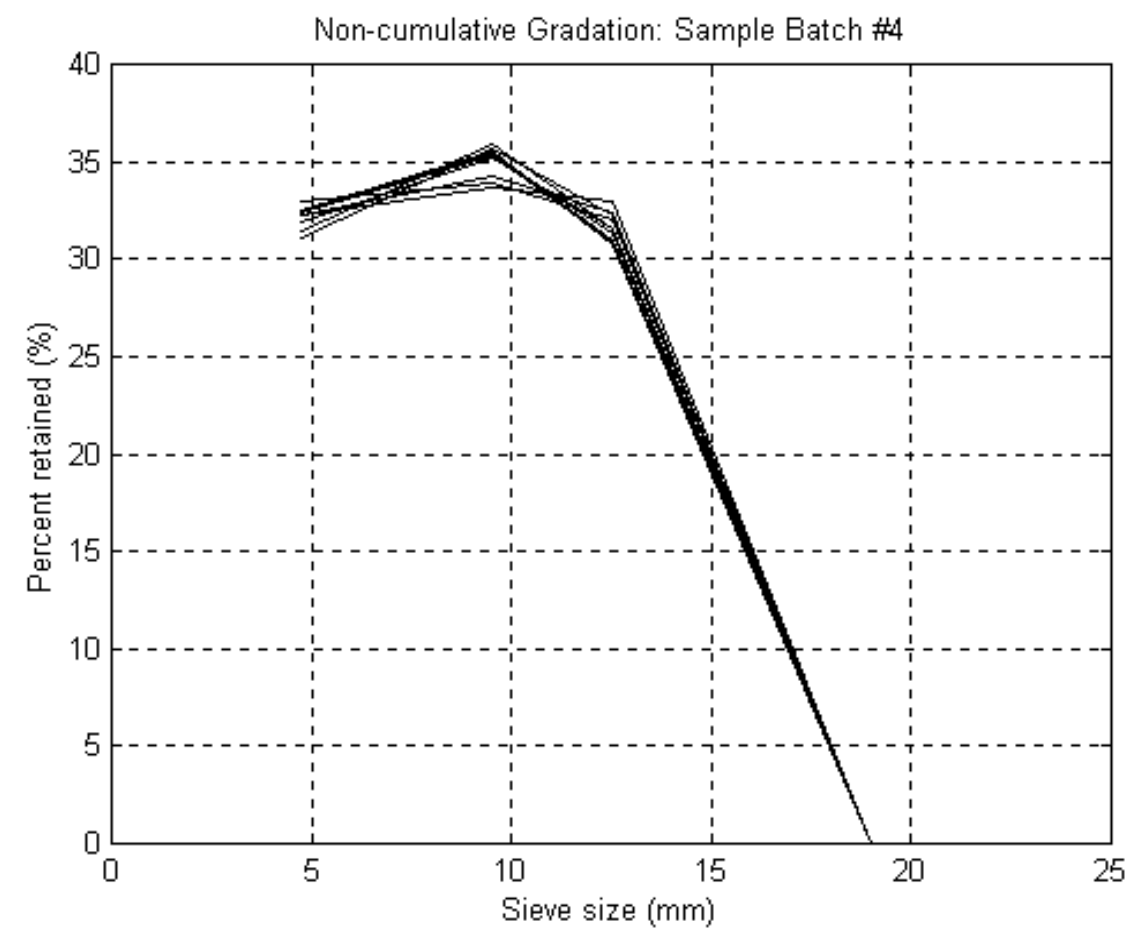

(a) percent retained 


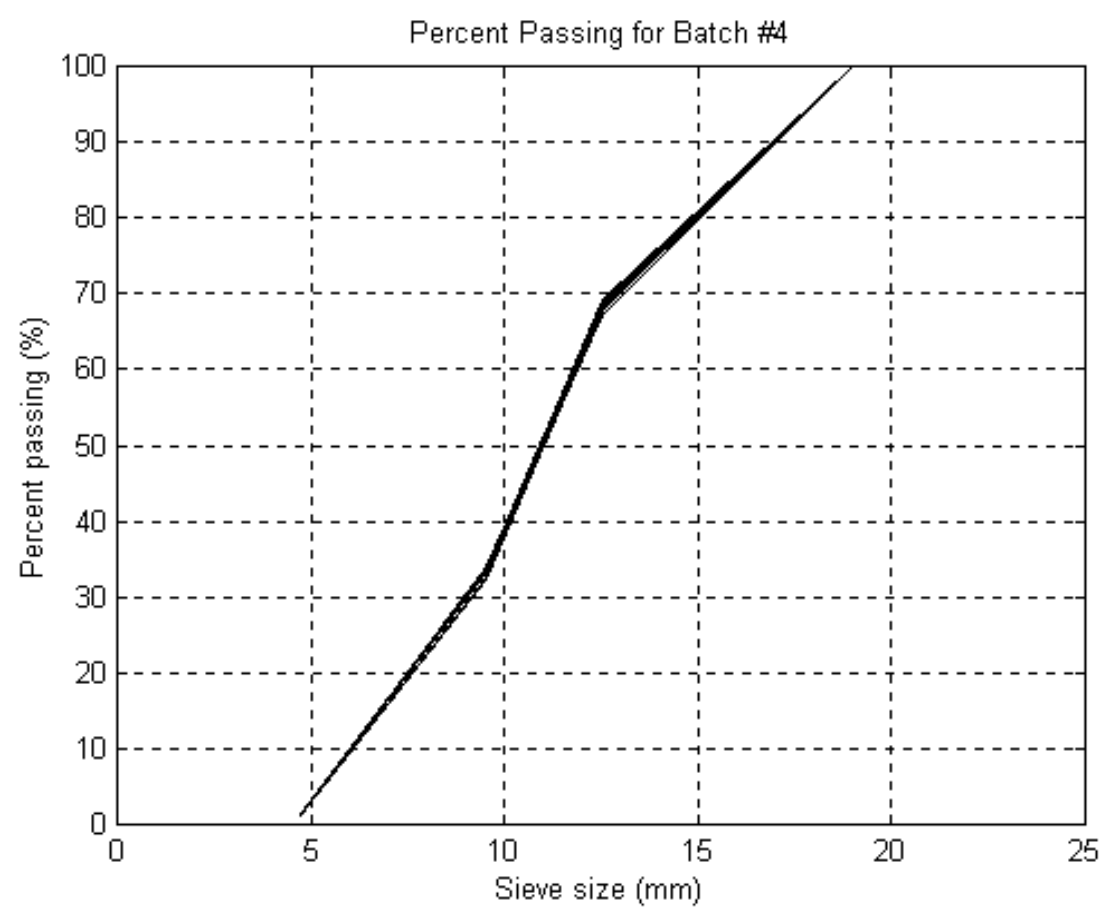

(b) percent passing

\section{Figure 7.11 Percent Retained and Percent Passing for Sample Batch \#4}

As expected, each resultant curve does not coincide with each other. In other words, ten times of mechanical sieving come up with ten different results. Two major reasons may explain these differences. First, borderline particles may only pass for a very particular orientation, which may or may not be achieved during the random tumbling of the sieving process. Secondly, attrition occurs during the sieving process. When all the particles are toppling in the enclosure of the vibrating and rotating sieves, particles are hitting and breaking down each other, inevitably reducing the size and volume of each particle. As a consequence, the mass in each sieve decreases as sieving goes on. This affects the value of percent retained, and thereby, affects the percent passing. Fig. 7.12 shows for sample batch $\# 7$ the data scattering for the $9.50 \mathrm{~mm}, 12.50$ $\mathrm{mm}$, and $19.00 \mathrm{~mm}$ sieve results. 


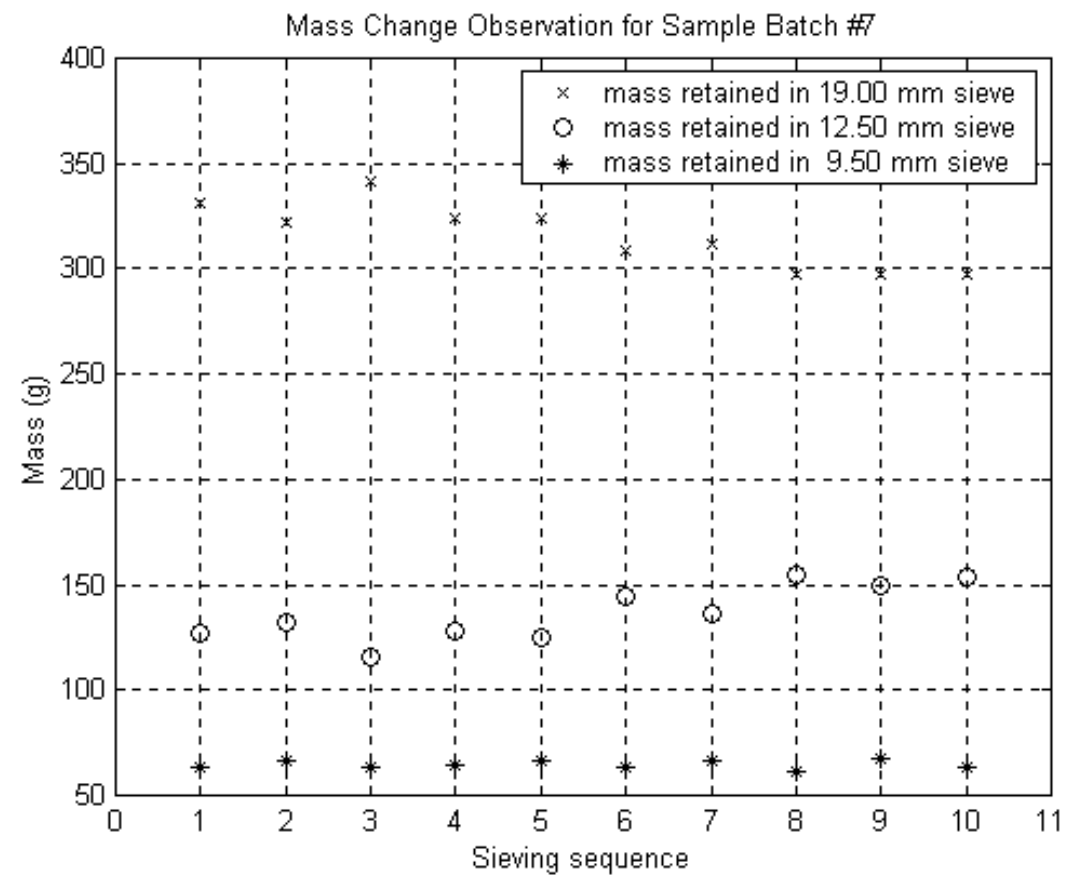

Figure 7.12 Mass Change in Sieving Process

To obtain the benchmark, the data points at each sieve size was averaged. Fig. 7.13 (a) (b) shows the benchmark of percent retained and percent passing, respectively, for sample batch \#4. 


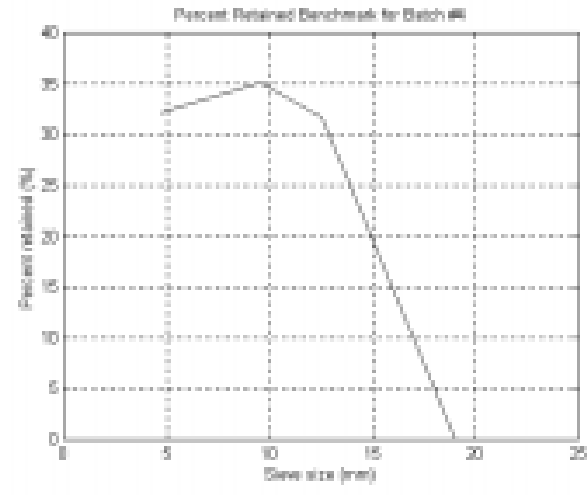

(a) percent retained

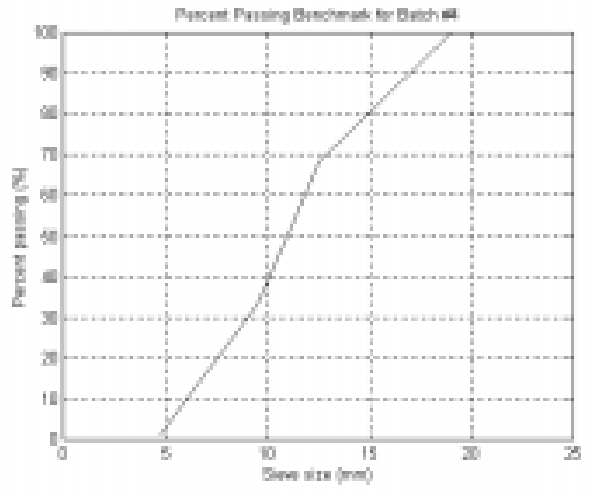

(b) percent passing

Figure 7.13 Benchmark for Sample Batch \#4

By the same method, the benchmark of all other batches was obtained, and given in Appendix III.

A sample population of larger size was formed by combining all the particles from the eight batches The corresponding percent retained and percent passing benchmark are shown in Fig. 7.14, and the values are listed in Table 7.2.

\subsection{Sieving Calibration Factor}

Using a large sample population formed by combining all eight batches, the sieving calibration factor, $\zeta_{s v}$, as introduced in inequality (7.10), was calibrated to be

$$
\zeta_{\mathbf{s v}}=\left[\begin{array}{c}
\zeta_{19.00} \\
\zeta_{1250} \\
\zeta_{950} \\
\zeta_{4.75}
\end{array}\right]=\left[\begin{array}{l}
2.00 \\
7.00 \\
3.00 \\
1.00
\end{array}\right]
$$

The calibration factors can be interpreted as follows:

The cross section of the particle is not rectangular. In most cases, the height at two ends of the minor diameter is shorter than the critical height $H_{\text {crit }}$, as illustrated 
previously in Fig. 7.4. This apparently increases the probability for the particles to pass the sieve. $\zeta_{\mathrm{sv}} \geq 1$ accounts for this rounding effect.

For each sieve of different size, there is a different calibration factor value. All these factors have been determined by back calculation from the large sample population with known size distribution. With the addition of these calibration factors, the sieving strategy's conditions expressed in inequality (7.10) becomes semi-empirical, because they are empirically derived values. The significance of $\zeta_{\mathrm{sv}}$ is that it accounts for numerous effects such as particle's cross section shape distribution. In summary, the physical significance is to account for combination of the following aspects:

1) The effect of the particle's cross section shape distribution. Although there is no theoretical a priori knowledge about cross section shape distribution inherent in the used method, it does affect the behavior of particles in the sieve to pass or be retained.

2) The effects of separation of overlapping and touching particles. Using the developed algorithm, the separated particles' shape and mass are not the same as they are manually isolated. This affects the particle's sieving behavior. Again, no a priori knowledge is available to determine even statistically how the sieving behavior will be influenced.

3) The effects caused by vision system errors such as hardware calibration, software imperfection.

4) The effects of sample population size. 


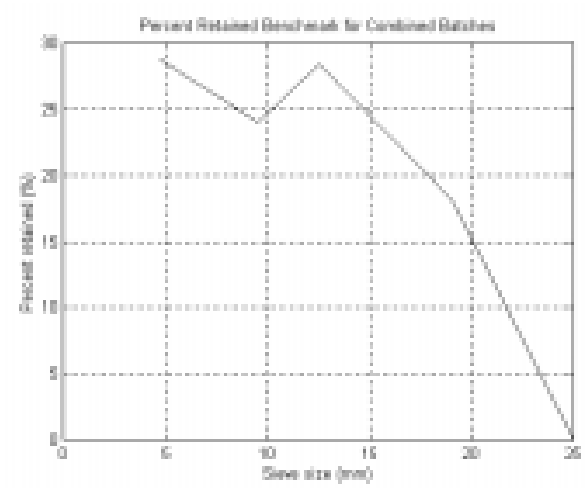

(a) percent retained

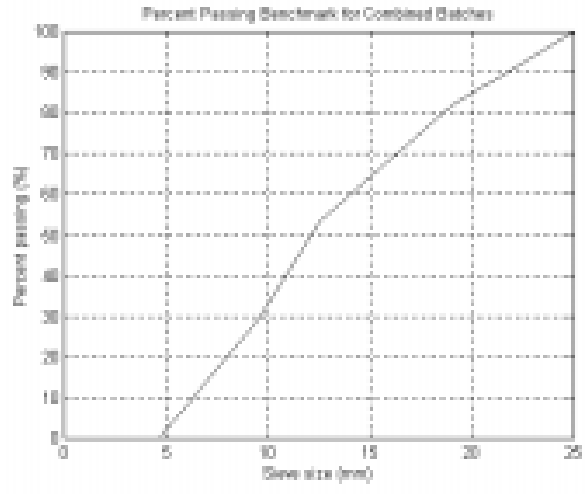

(b) percent passing

Figure 7.14 Benchmark for Combined Sample Batches

Table 7.2 Benchmark Values for Combined Sample Batches

\begin{tabular}{|l|l|l|l|l|l|}
\hline \multirow{2}{*}{ Type } & \multicolumn{5}{c|}{ Sieve Size (mm) } \\
\cline { 2 - 6 } & 4.75 & 9.50 & 12.50 & 19.00 & 25.00 \\
\hline $\begin{array}{l}\text { Percent } \\
\text { Retained (\%) }\end{array}$ & 28.806 & 23.996 & 28.390 & 18.250 & 0.000 \\
\hline $\begin{array}{l}\text { Percent } \\
\text { Passing (\%) }\end{array}$ & 0.557 & 29.383 & 53.385 & 81.766 & 100 \\
\hline
\end{tabular}

\subsection{Vision Sieving Result}

With the calibrated $\zeta_{s v}$ 's, sieving correlation testing conducted. The samples are the same as those used for volumetric model testing, i.e., eight batches with a total of 1862 particles. Recall that the benchmark has been available, as shown in Fig. 7.14. The basic algorithm was written in Table 7.1

Again, a random combination of the images was taken, each of combination's constituent image was randomly selected from one different sample batch. This indicates that the total number of particles will increase up to $N=N_{1}+N_{2}+\ldots+N_{8}=1862$, which is a 
summation of particle number of all eight batches. Fig. 7.15 illustrates how a random combination of photo $d, a, b, e, b, d, c, a$ selected from sample batch \#1, \#2, ..., \#8 respectively, is formed. 


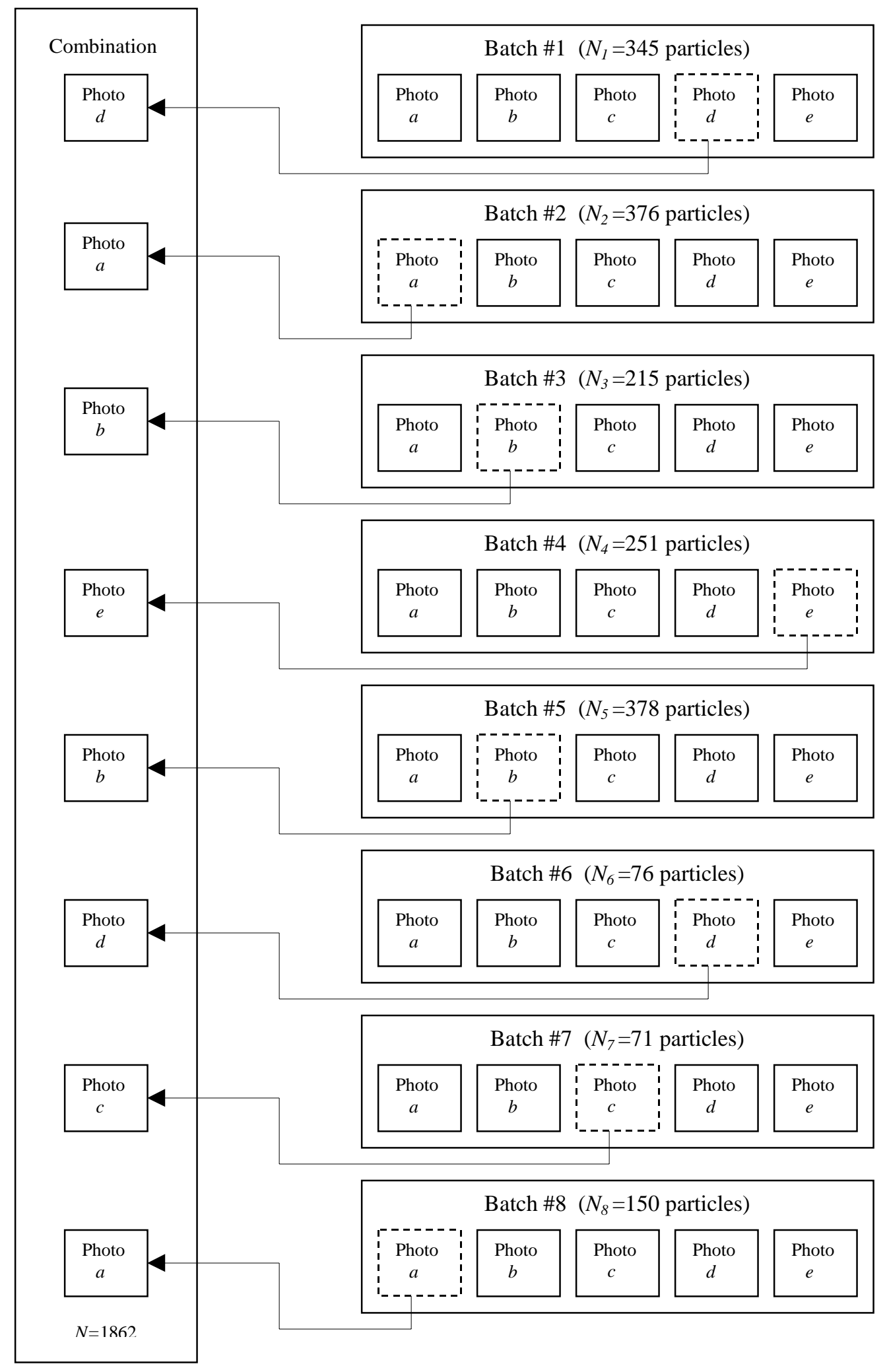

Figure 7.15 A Random Combination of Sample Batches (dabebdca) 
In effect, each random combination represents one "grand" image covering all 1862 particles. Each constituent image results from a new "shuffling" of the same sample batch. Therefore, each random combination of images may be regarded as result of "grand shuffling" of 1862 particles.

Testing on five grand images was conducted. This means that 1862 particles were shuffled five times to produce these five grand images. On each grand image, all particles were "optically" sieve ten times, final vision sieving result is taken by averaging these ten results, and then compare it to the benchmark.

Figures from Fig. 7.16 to Fig. 7.20 demonstrate the results of these five testings. From the results, it shows that the percent passing residuals are within $\pm 3 \%$. The residual between the percent passing benchmark and vision sieving percent passing is listed in Table 7.3. The error may be contributed to by the following reasons:

1) Simple measurement of the particle minor diameter is not an entirely true representation of what happens in physical sieving. Particles that are somewhat flat can turn diagonally in the sieve and pass a smaller mesh size than one would suspect from simple size measurements.

2) Error in the calibration factor $\mathrm{mm} /$ pixel can contribute to vision sieving error.

3) Accurate measurement of percent passing requires accurate projection of the volume from the optically measured parameters. This in practice is impossible to achieve with extreme accuracy, especially for individual particles.

4) Error existing in minor diameter estimation for triangular shapes can contribute to sieving correlation error. 
5) Overlapping and touching particles' profile shape and volume estimate change during separation.

6) If the size sample population is not sufficiently large, it will create error in vision sieving process because a uniformly distributed random number is involved in determining "pass or retain" for a particle in the sieve.

7) The benchmark itself has certain discrepancies due to particle physical sieving behavior. 


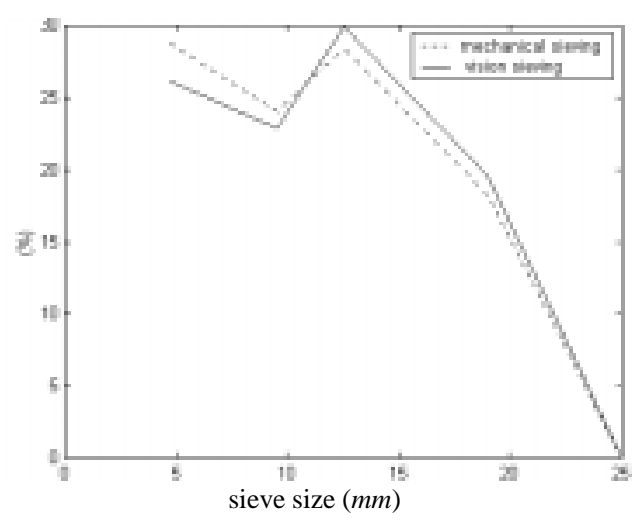

(a) percent retianed

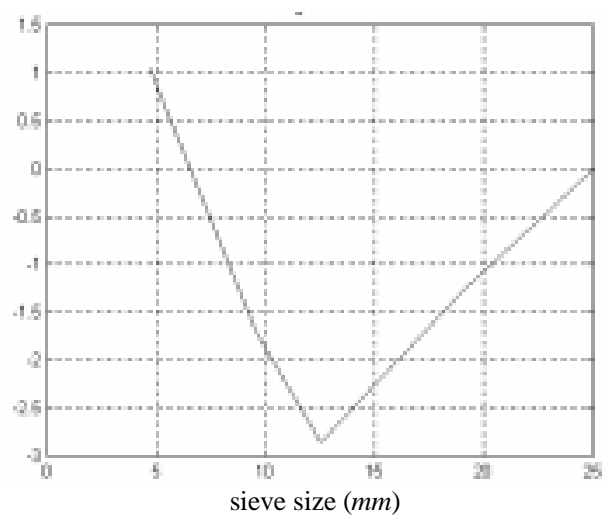

(b) percent passing residual

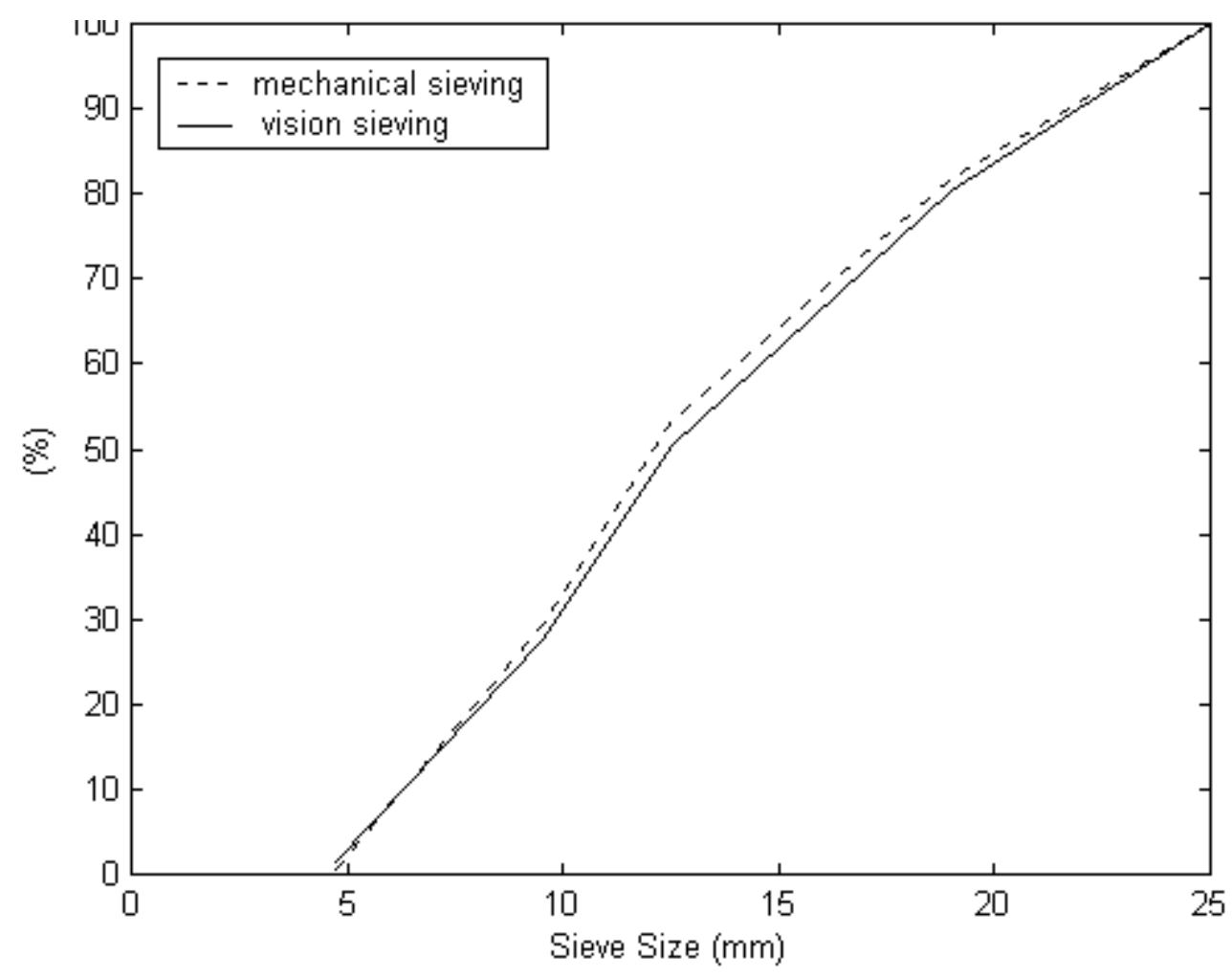

(c) cumulative percent passing

Figure 7.16 Sieving Correlation Testing \#1

(Combination: image e,e,d,b,c,d,c,e from sample batch \#1 to \#8, respectively) 


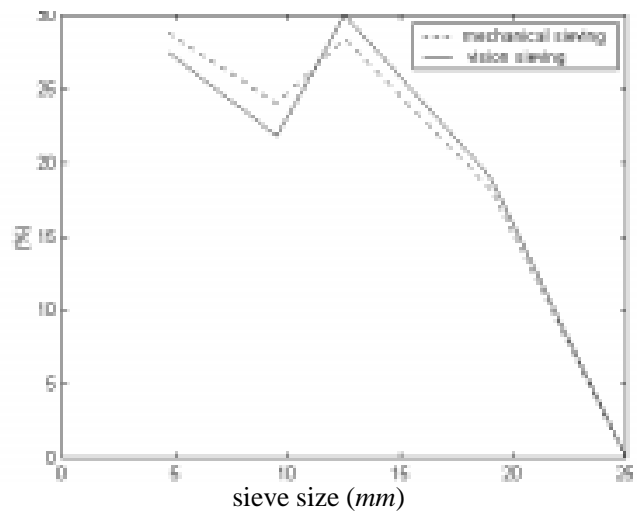

(a) percent retained

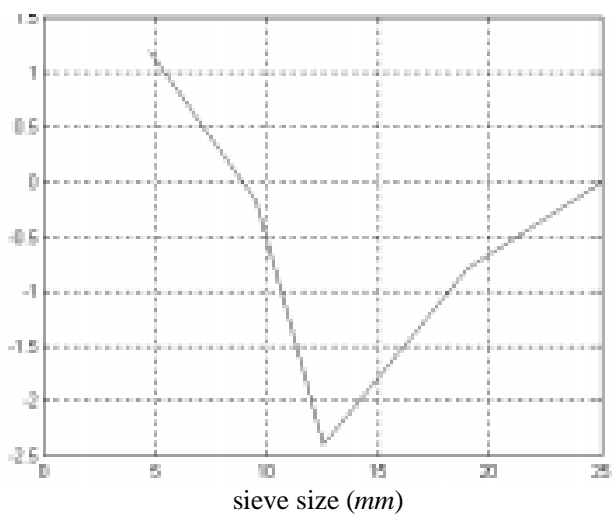

(b) percent passing residual

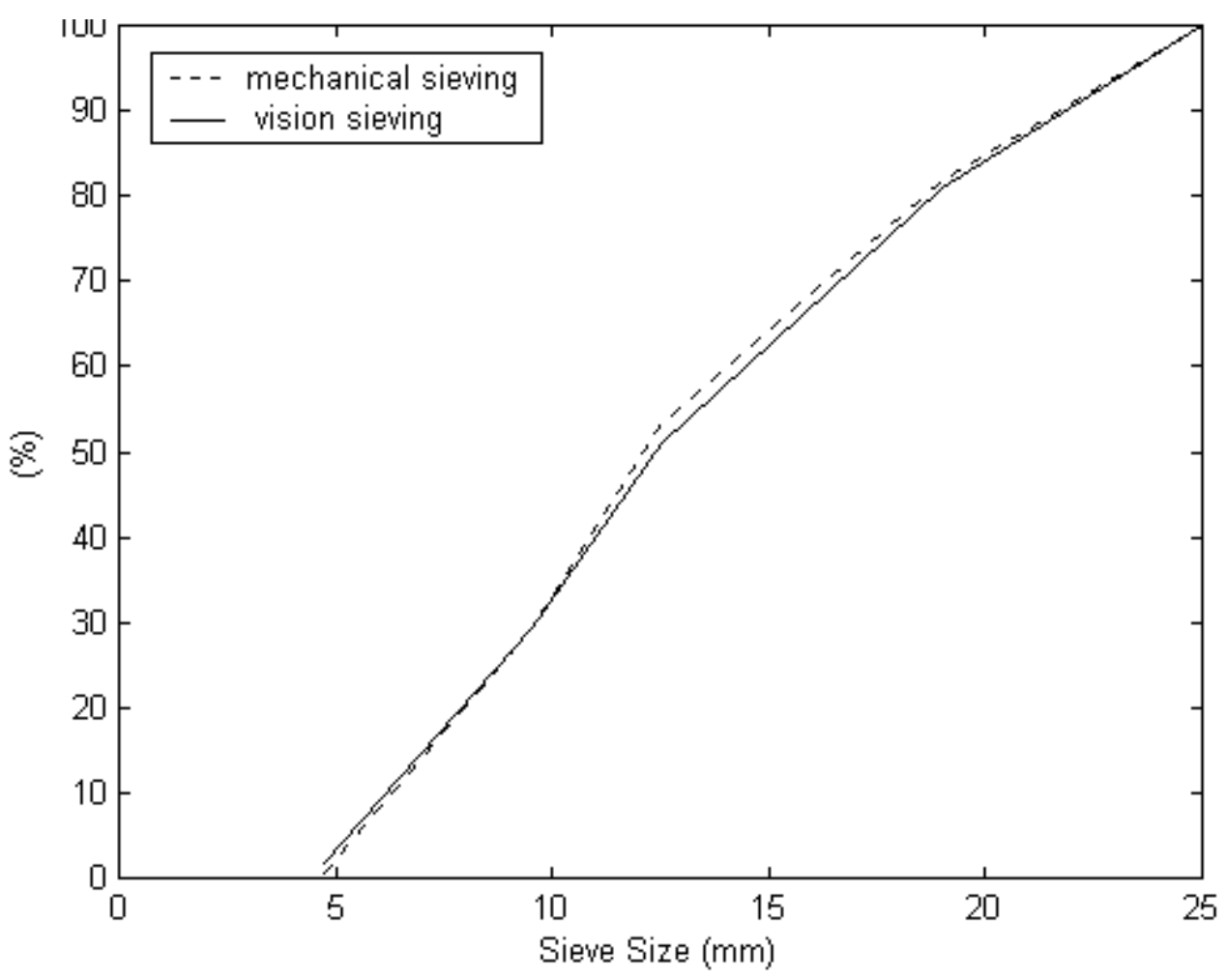

(c) cumulative percent passsing

Figure 7.17 Sieving Correlation Testing \#2

(Combination: image c,e,a,c,a,b,d,e from sample batch \#1 to \#8, respectively) 


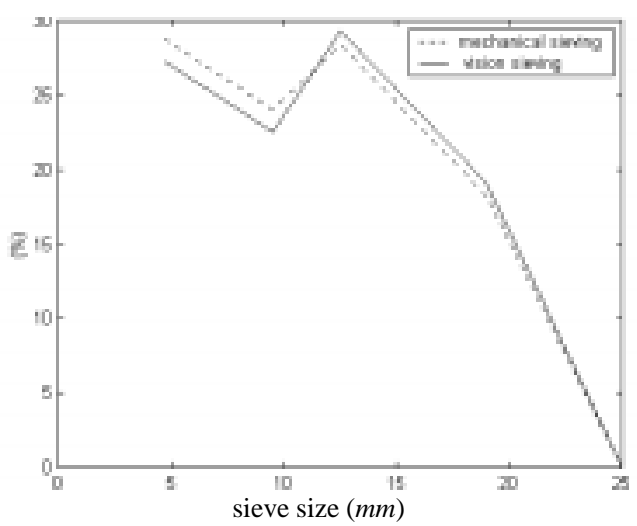

(a) percent retained

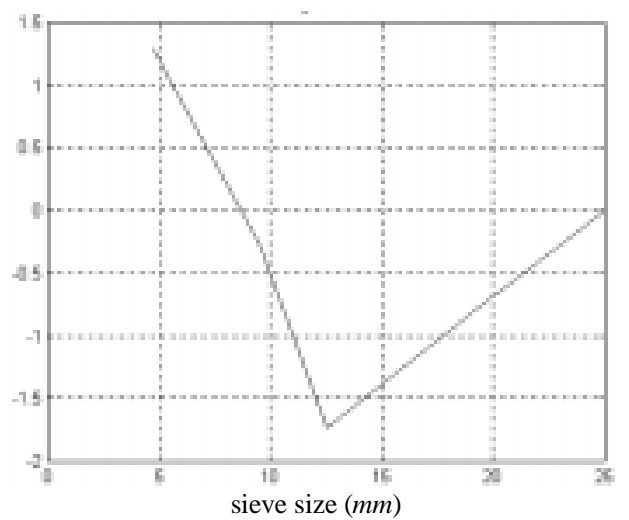

(b) percent passing residual

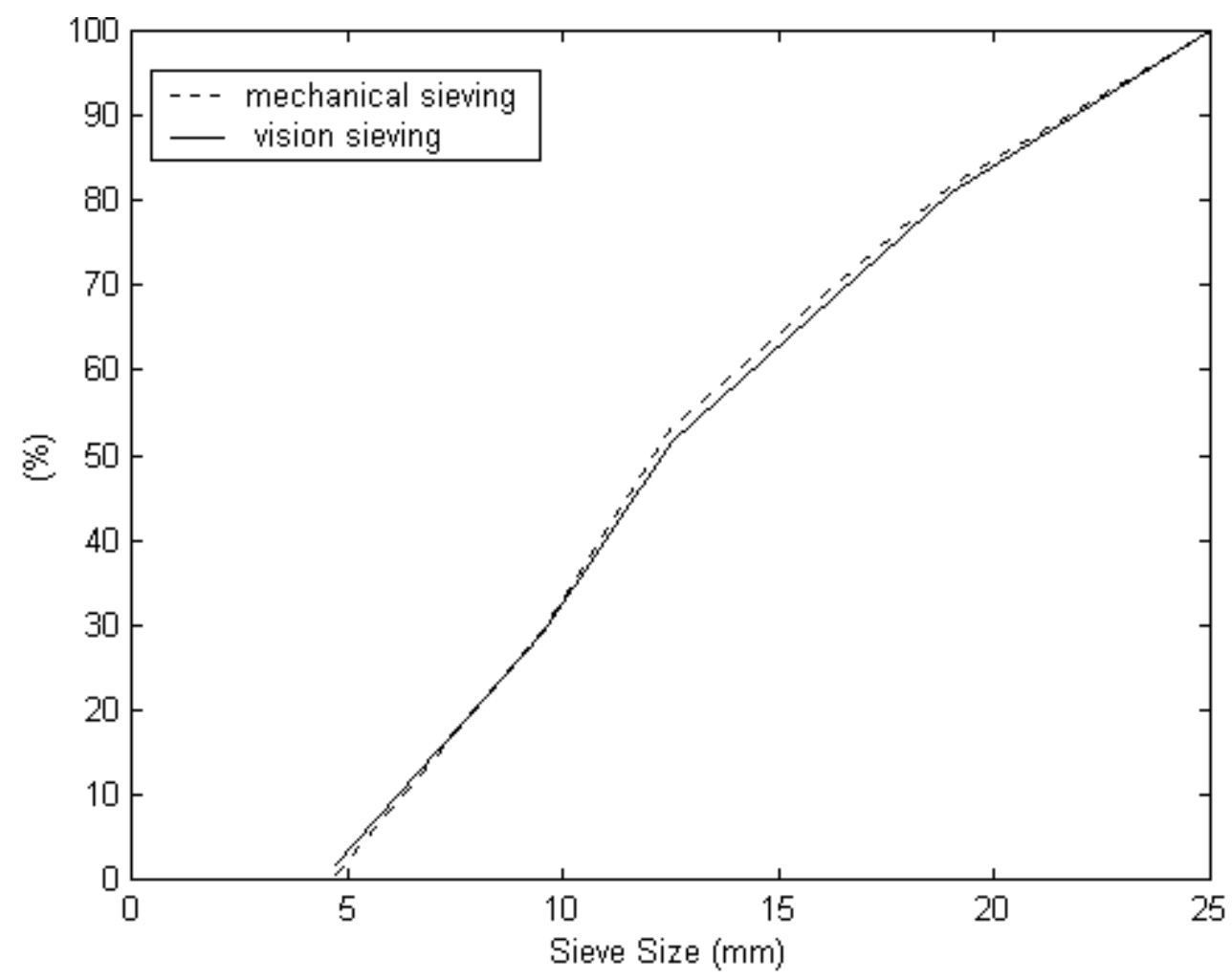

(c) cumulative percent passsing

Figure 7.18 Sieving Correlation Testing \#3

(Combination: image e,d,e,a,a,a,b,c from sample batch \#1 to \#8, respectively) 


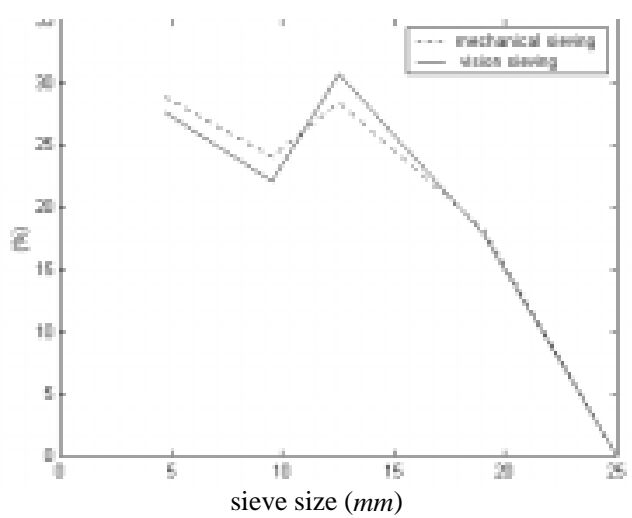

(a) percent retained

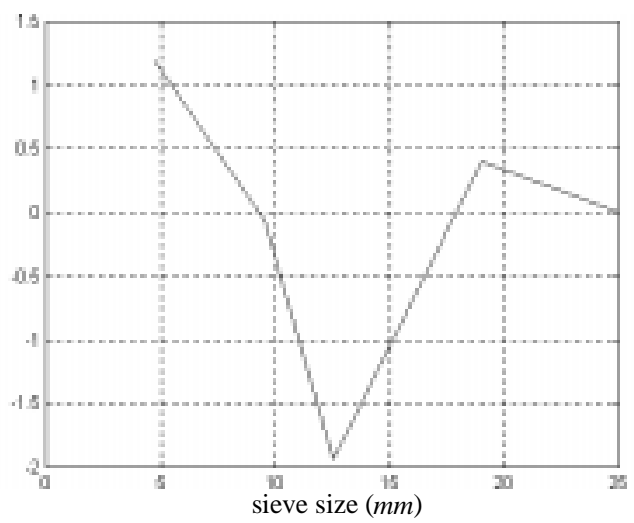

(b) percent passing residual

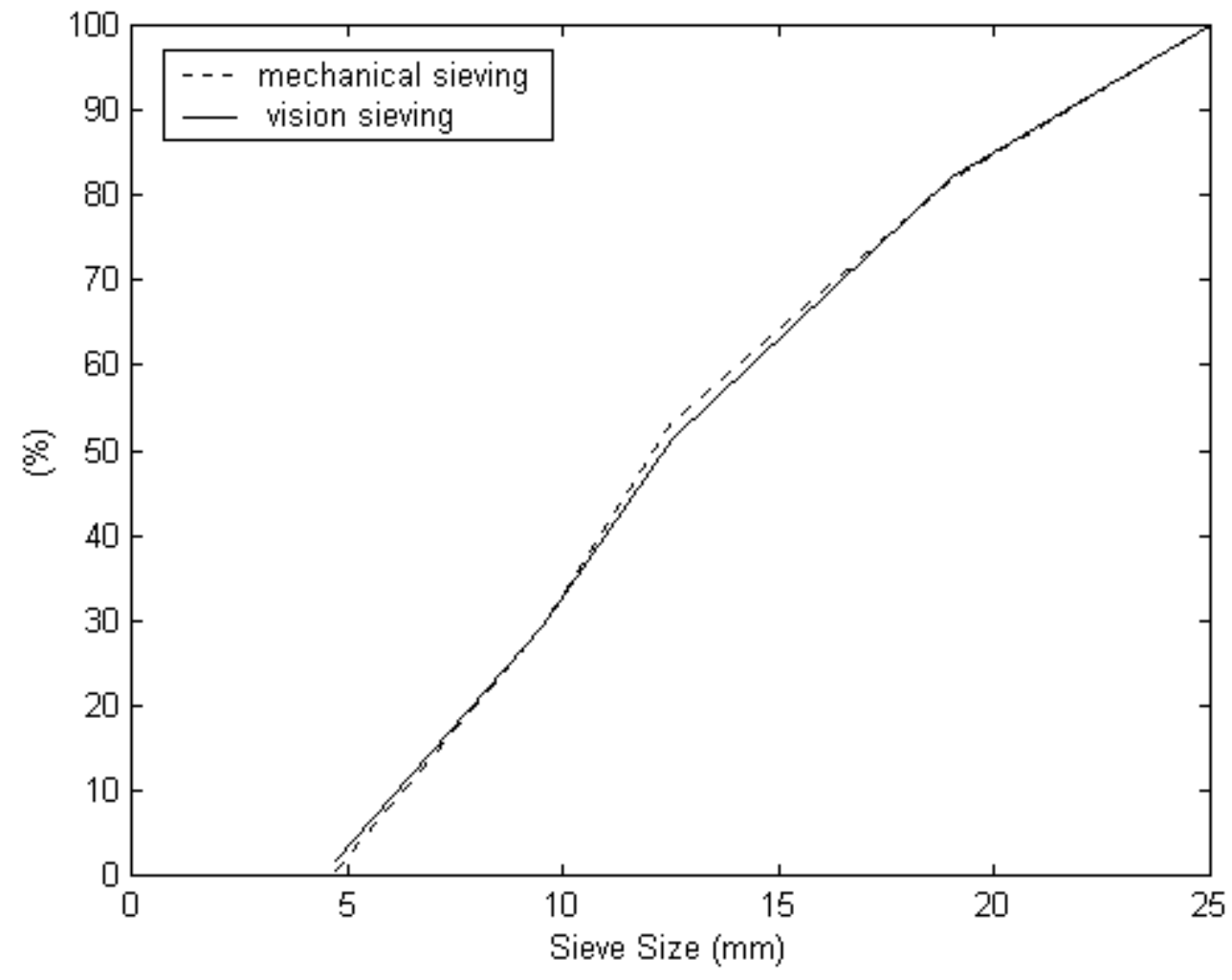

(c) cumulative percent passsing

Figure 7.19 Sieving Correlation Testing \#4

(Combination: image b,e,c,e,a,e,e,e from sample batch \#1 to \#8, respectively) 


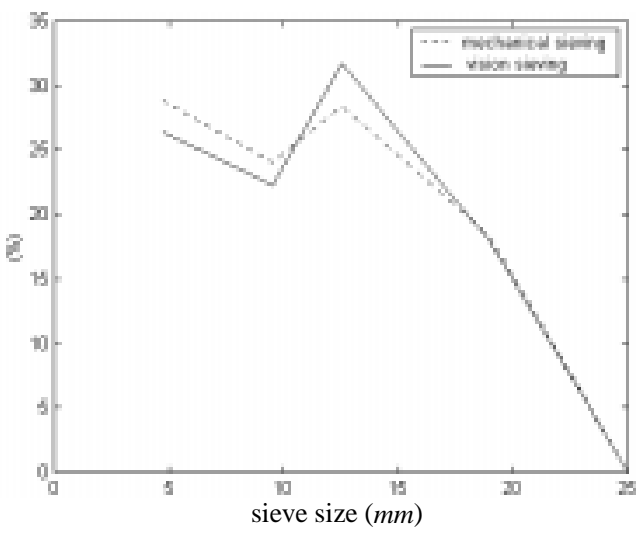

(a) percent retained

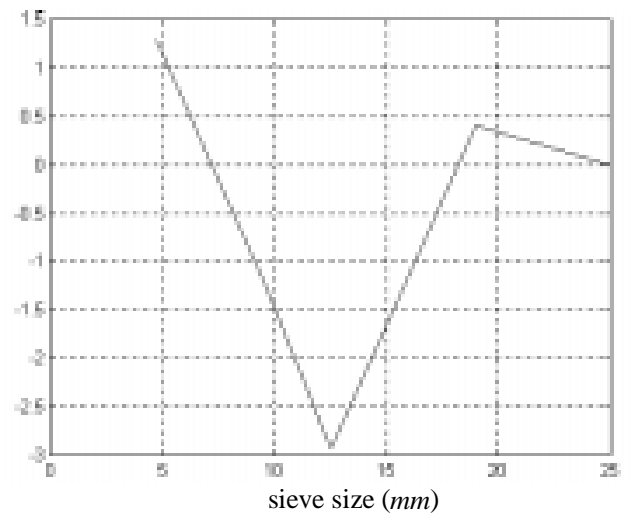

(b) percent passing residual

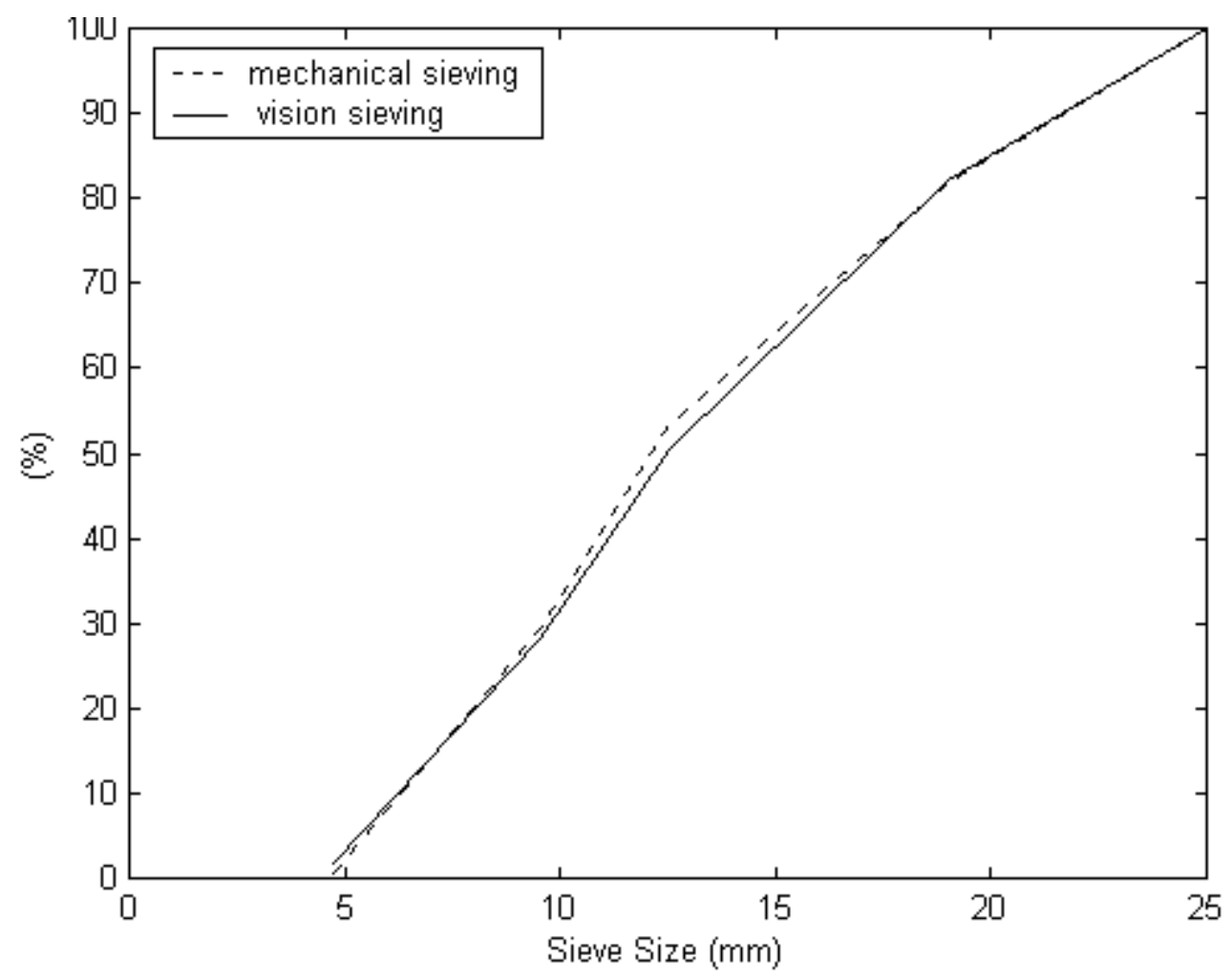

(c) cumulative percent passsing

Figure 7.20 Sieving Correlation Testing \#5

(Combination: image e,d,d,a,c,e,d,b from sample batch \#1 to \#8, respectively) 
Table 7.3 Testing Residuals

\begin{tabular}{|c|l|l|l|l|l|}
\hline \multirow{2}{*}{$\begin{array}{c}\text { Test } \\
\text { number }\end{array}$} & \multicolumn{5}{|c|}{ Sieve Size (mm) } \\
\cline { 2 - 6 } & 4.75 & \multicolumn{5}{|c|}{ Percent-Passing Residual (\%) } \\
\hline$\# 1$ & 1.0406 & -1.6774 & -2.8493 & -1.2805 & 0.0000 \\
\hline$\# 2$ & 1.1905 & -0.1776 & -2.3980 & -0.7918 & 0.0000 \\
\hline$\# 3$ & 1.2950 & -0.2743 & -1.7304 & -0.8218 & 0.0000 \\
\hline$\# 4$ & 1.1915 & -0.0563 & -1.9429 & 0.4042 & 0.0000 \\
\hline$\# 5$ & 1.2828 & -1.1989 & -2.9305 & 0.3992 & 0.0000 \\
\hline $\begin{array}{c}\text { Mean } \\
\text { Error }\end{array}$ & 1.2001 & -0.6769 & -2.3702 & 0.4181 & 0.0000 \\
\hline
\end{tabular}




\section{SYSTEM PERFORMANCE}

\subsection{Introduction}

The testing results that have been obtained thus far were from the sample population in which all the particles were separated manually. In practice, it is implausible to manually arrange a large number of particles prior to being imaged.

In this chapter, a sample population was arranged in such a way that particle touching and overlapping occurrences were allowed. The touching and overlapping particles are separated using the separation algorithm described in chapter 5, then go through the same process of image analysis as did the samples previously. The benchmark of percent passing for the sample tested is obtained from the WVU civil engineering laboratory. By comparison of the optical sieving result and the benchmark, the performance of the system is evaluated.

\subsection{Sample Preparation and Discussion}

A sample of population of $N=1972$ particles was prepared for testing the performance of the developed system. These particles were broken down into ten sample groups due to backlight panel dimension limitation. In each sample group, touching and overlapping were allowed among particles. For sample group \#2, Fig. 8.1 (a) shows the binary images with some occurrences of touching and overlapping of limited extent, while (b) shows the corresponding image in which separation was completed. The similar figures for all ten groups are given in Appendix IV. 


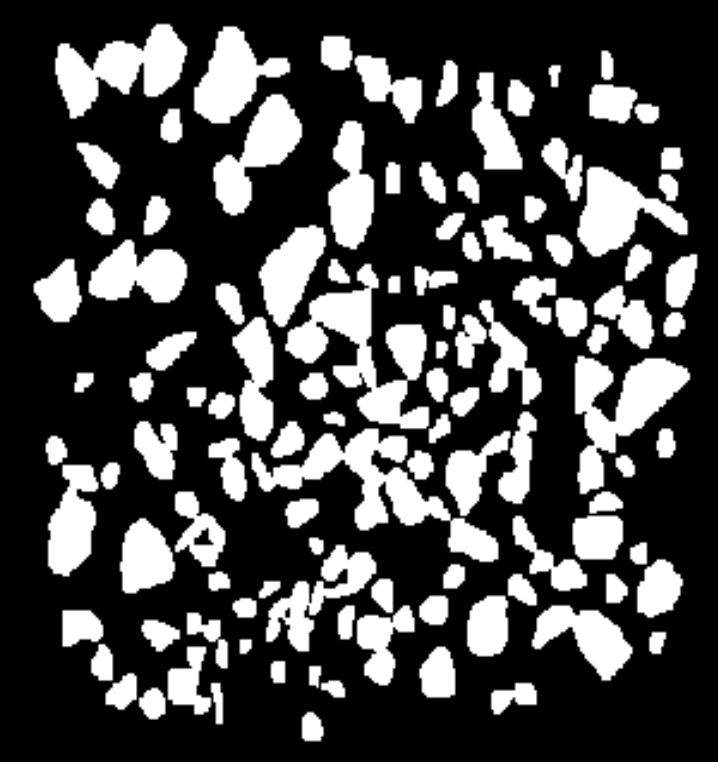

(a) group \#2: particles with touching and overlapping

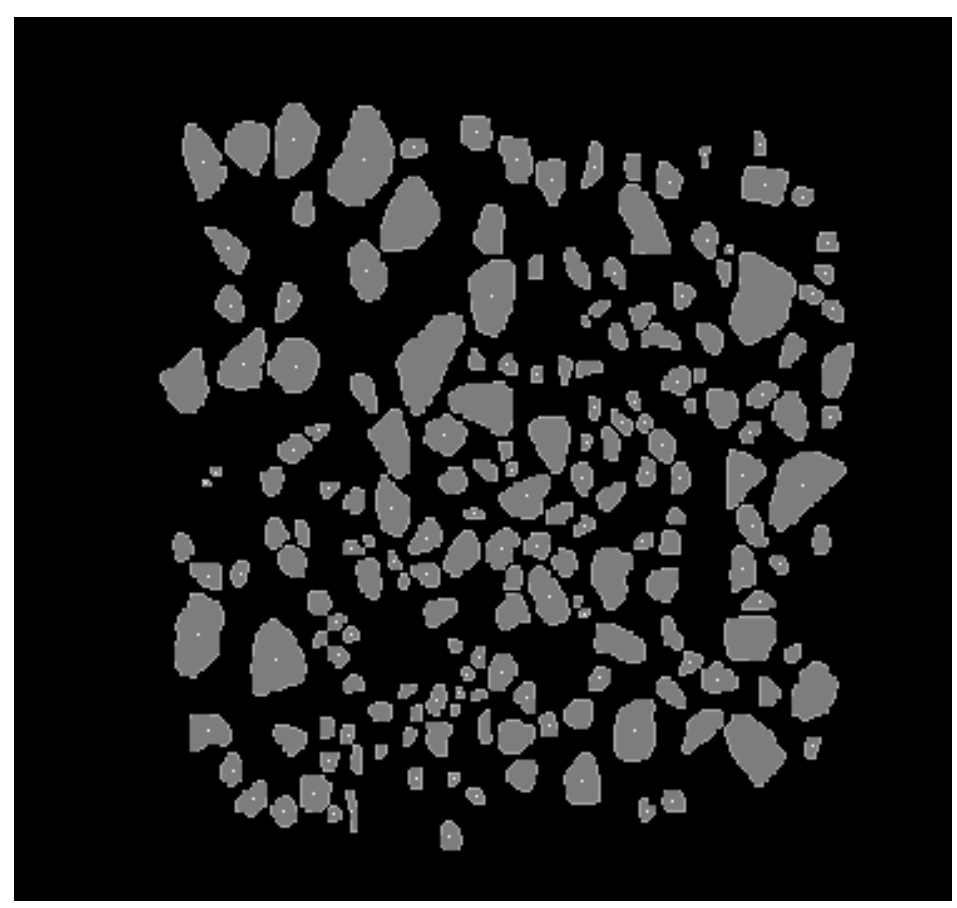

(b) group \#2: particles after separation

Figure 8.1 Particles of Sample Group \#2 
Some effects caused by separation can be observed after applying the algorithm to such a large number of particles. For a few particles, "false cutting" took place, mainly for particles with a concave shape. This increases the total number of particles retained in smaller sieves. "Miscutting" was noted among a very small number of particles. By miscutting, it means that the connected particles are separated, but not at the place they should be. The reason for miscutting is complex, and is definitely related to the contour shape of the connected particles. In most cases, the separating process appears satisfactory. The impact on the sieving results caused by separation is assumed to be insignificant.

To gain some statistical perception of the sample population tested, the histogram of optically measured minor diameter, estimated flatness, and estimated volume are presented in Fig. 8.2, Fig. 8.3, and Fig. 8.4, respectively.

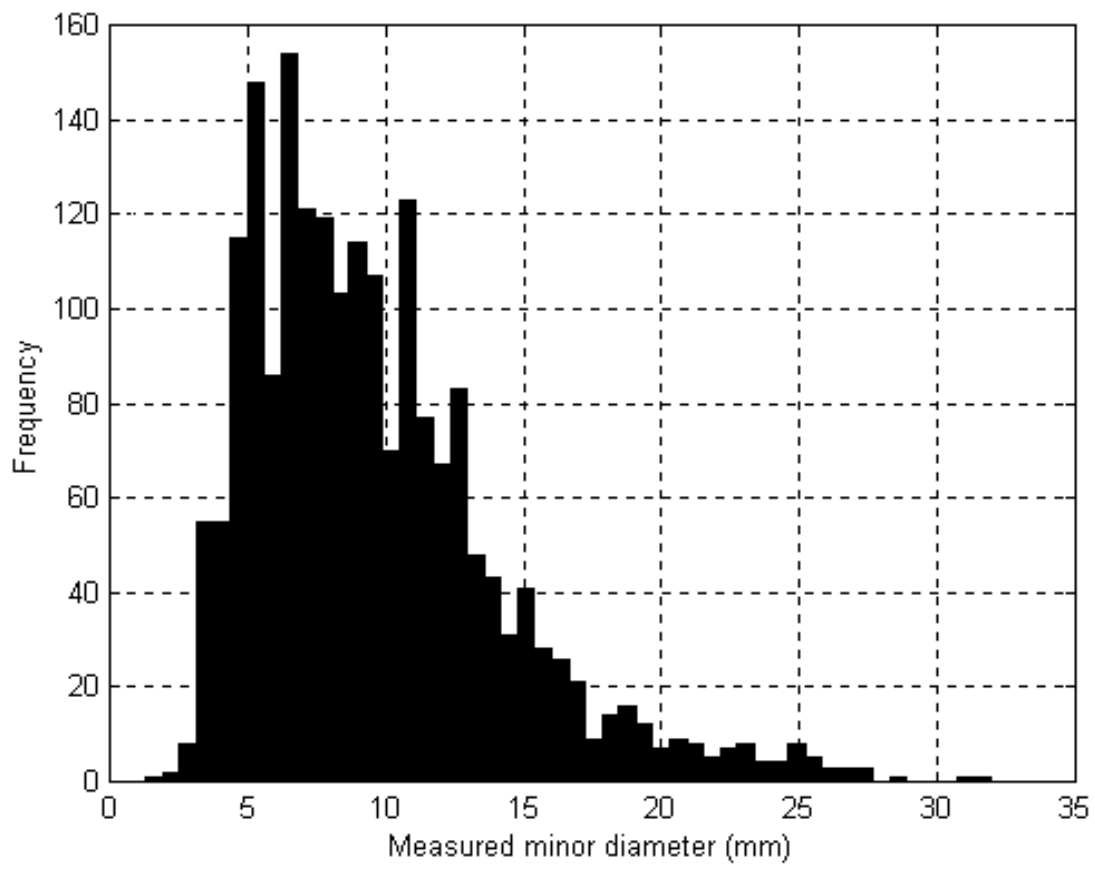

Figure 8.2 Histogram of Measured Minor Diameter 


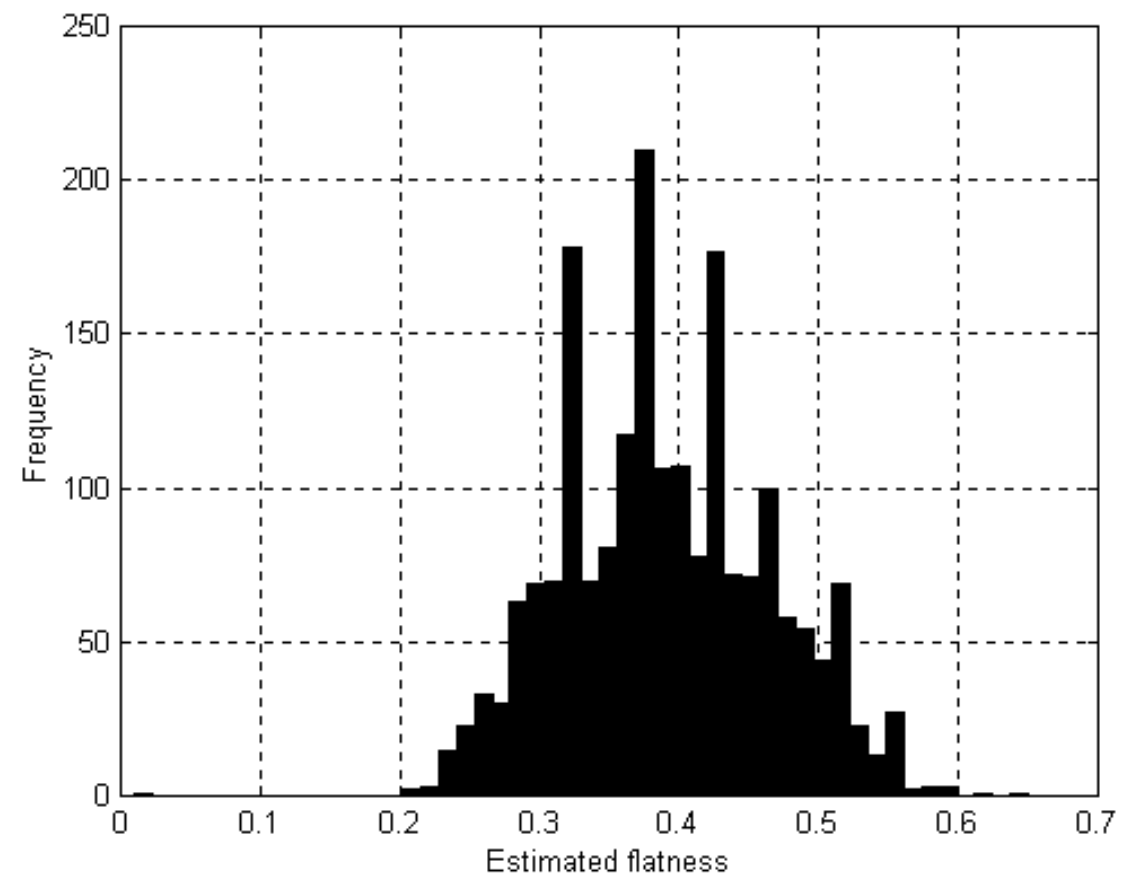

Figure 8.3 Histogram of Estimated Flatness

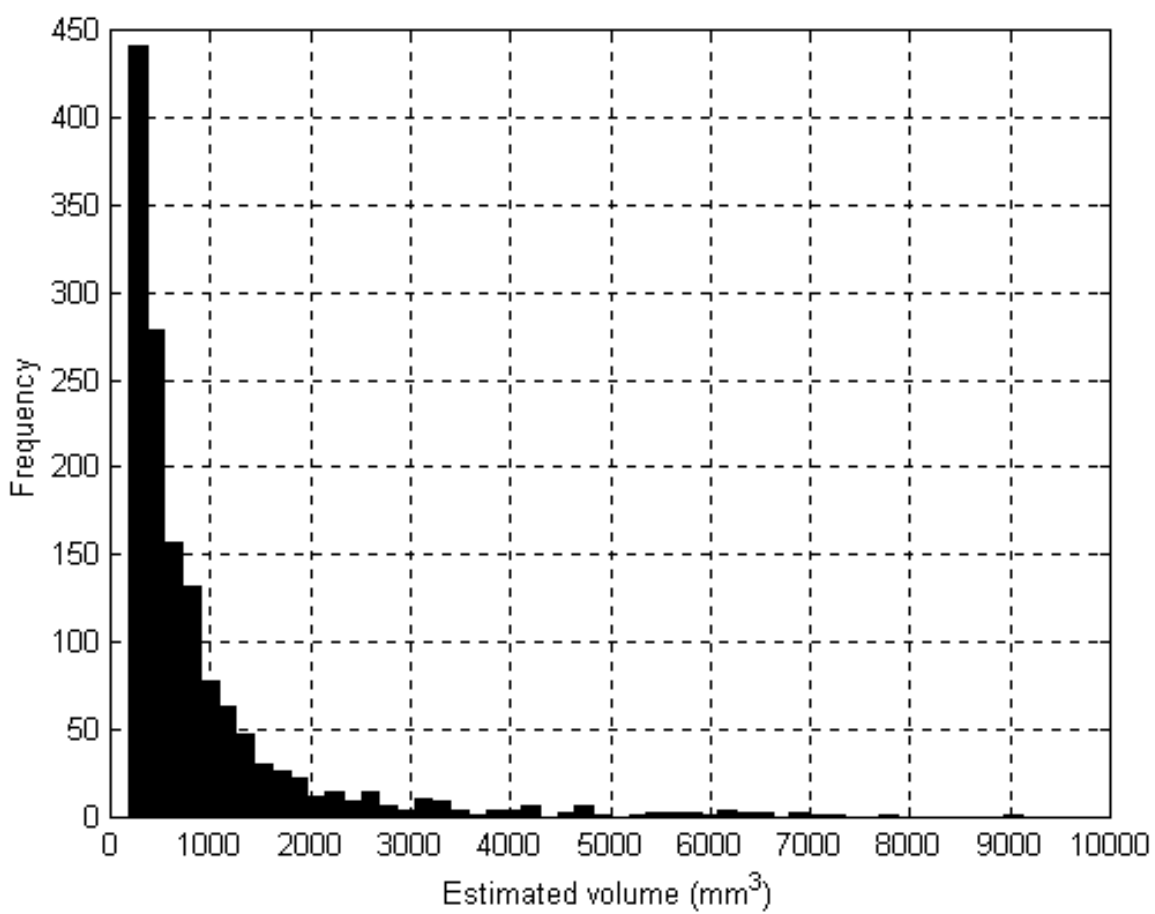

Figure 8.4 Histogram of Estimated Volume 


\section{3 $\underline{\text { Sample Benchmark }}$}

The sample of 1972 particles was mechanically sieved ten times in the laboratory. Each time the sieving result is expectedly different. The causes of these differences were described in section 7.6. Fig. 8.5 (a) shows the percent retained curves from the ten sievings, and (b) is the benchmark that is averaged from these ten values at each sieve size. Correspondingly, Fig. 8.6 (a) shows the ten percent passing curves, and (b) works as the percent passing benchmark for the sample population that will be sieved optically.

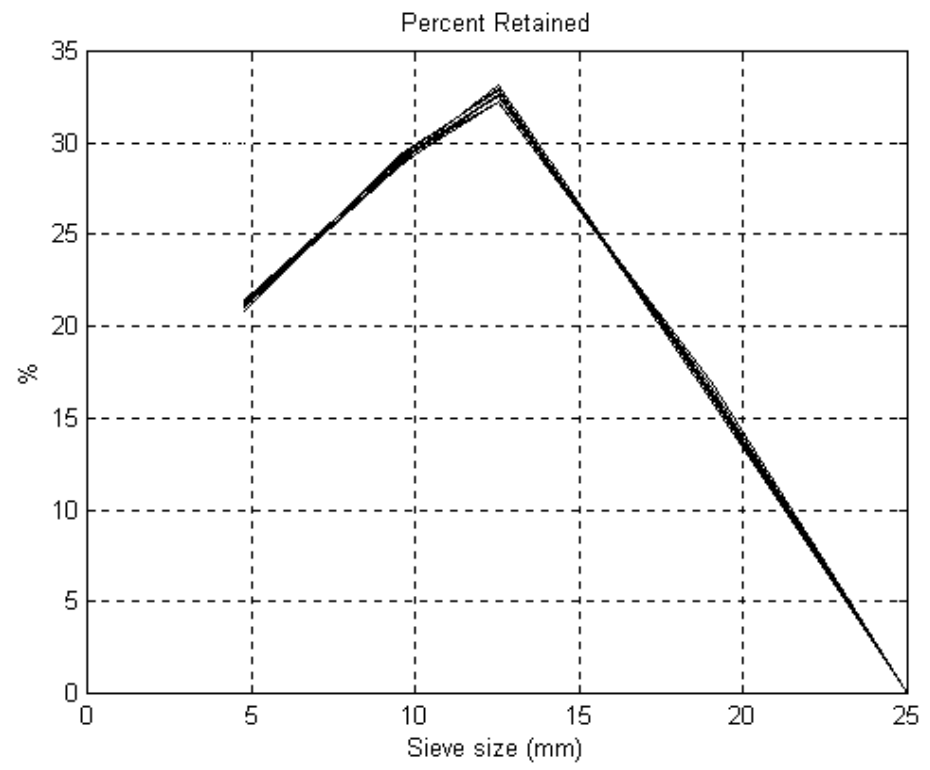

(a) percent retained curves for sieving 10 times 


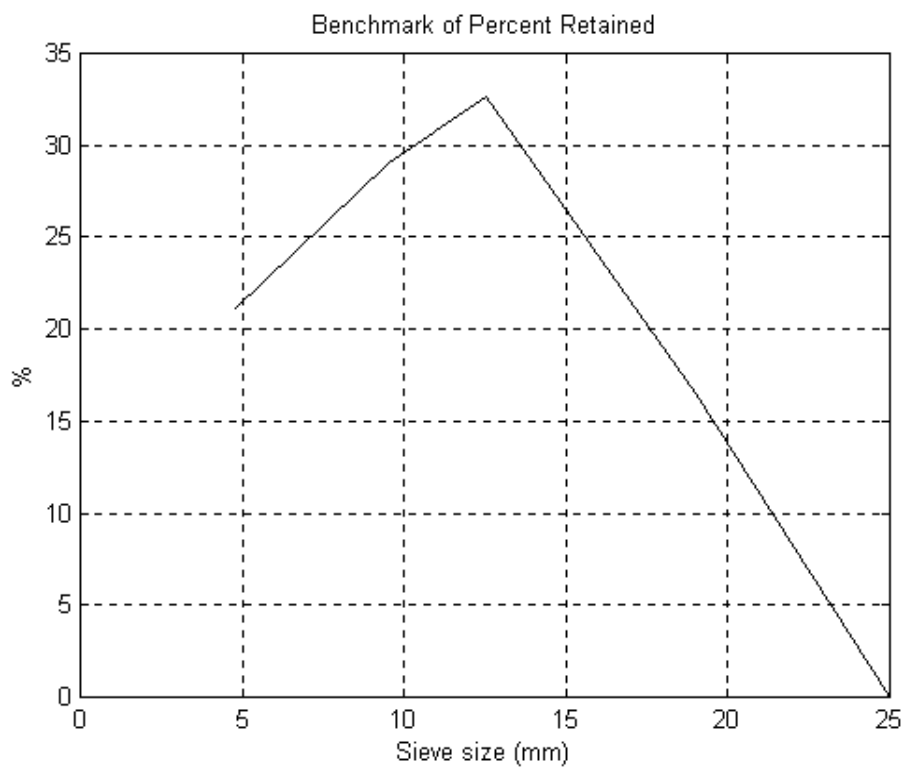

(b) averaged curve as the benchmark

Figure 8.5 Percent Retained Benchmark

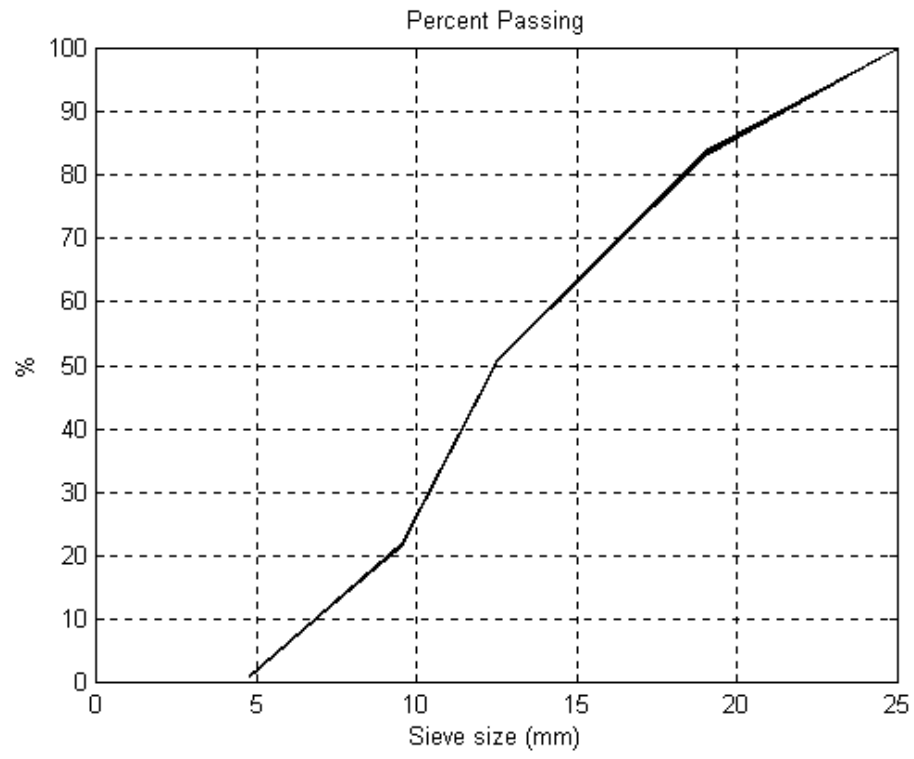

(a) percent passing curves for sieving 10 times 


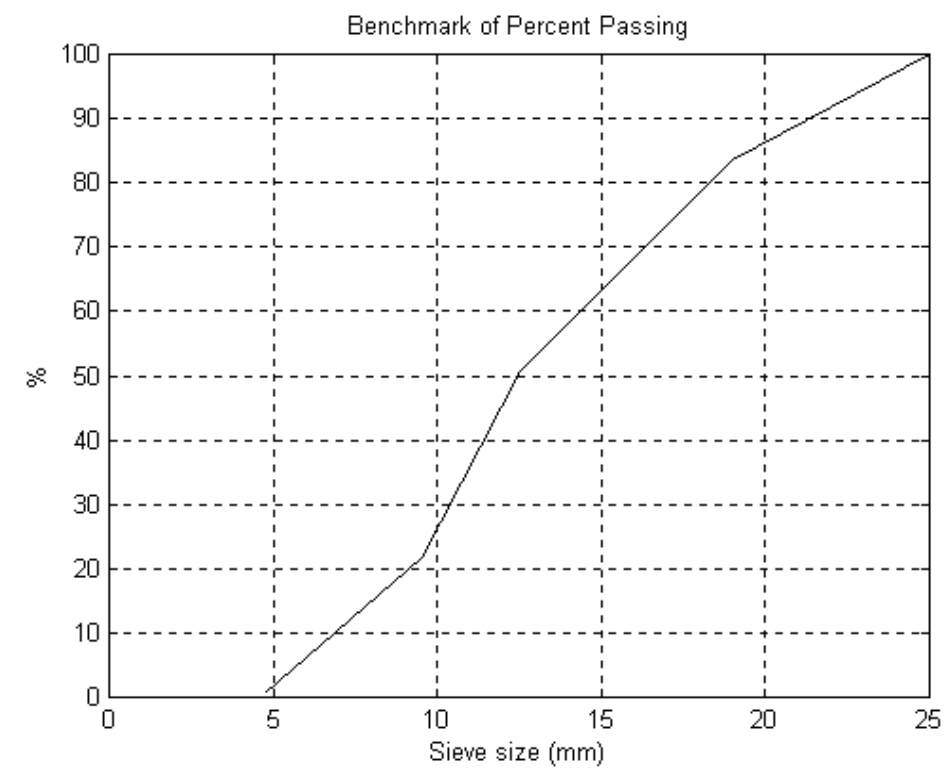

(b) averaged curve as the benchmark

Figure 8.6 Percent Passing Benchmark

The statistics of the mechanical sieving is tabulated in the table below.

Table 8.1 Statistics of the Benchmark (Sieving of 10 times)

\begin{tabular}{|c|c|c|c|c|c|}
\hline \multicolumn{3}{|c|}{$\begin{array}{c}\text { Percent Retained } \\
(\%)\end{array}$} & \multicolumn{3}{c|}{$\begin{array}{c}\text { Percent Passing } \\
(\%)\end{array}$} \\
\hline $\begin{array}{c}\text { Sieve size } \\
(\mathrm{mm})\end{array}$ & Mean & Variance & $\begin{array}{c}\text { Sieve size } \\
(\mathrm{mm})\end{array}$ & Mean & Variance \\
\hline 25.00 & 0.00 & 0.00 & 25.00 & 100.00 & 0.00 \\
\hline 19.00 & 16.51 & 0.10 & 19.00 & 83.49 & 0.10 \\
\hline 12.50 & 32.65 & 0.08 & 12.50 & 50.84 & 0.03 \\
\hline 9.50 & 29.06 & 0.03 & 9.50 & 21.78 & 0.02 \\
\hline 4.75 & 21.06 & 0.03 & 4.75 & 0.72 & 0.00 \\
\hline
\end{tabular}




\subsection{Results Comparison}

To establish confidence in the optical sieving system, the technique must yield results readily comparable to mechanical sieving methods.

For these separated 1972 particles, the same sieving procedure was used as had been for those samples described in section 7.8. The same sieving calibration factors were used. The sample was "optically" sieved 10 times, and the mean of the results was taken as the test value.

The results are listed in Table 8.2 and Table 8.3. Fig. 8.7 and Fig. 8.8 show the graphical comparison.

Table 8.2 Percent Retained Result Comparison

\begin{tabular}{|c|c|c|c|c|c|c|c|c|}
\hline & \multicolumn{8}{|c|}{ Sieve Size $(\mathrm{mm})$} \\
\hline & \multicolumn{2}{|c|}{4.75} & \multicolumn{2}{|c|}{9.50} & \multicolumn{2}{|c|}{12.50} & \multicolumn{2}{|c|}{19.00} \\
\hline \multirow{4}{*}{$\begin{array}{l}\text { Test } \\
\text {-ing }\end{array}$} & \multicolumn{8}{|c|}{ Benchmark Value (\%) } \\
\hline & \multicolumn{2}{|c|}{21.06} & \multicolumn{2}{|c|}{29.06} & \multicolumn{2}{|c|}{32.65} & \multicolumn{2}{|c|}{16.51} \\
\hline & \multicolumn{8}{|c|}{ Comparison and Residual } \\
\hline & $\begin{array}{l}\text { Testing } \\
\text { result }\end{array}$ & Residual & $\begin{array}{c}\text { Testing } \\
\text { result }\end{array}$ & Residual & $\begin{array}{c}\text { Testing } \\
\text { result }\end{array}$ & Residual & $\begin{array}{l}\text { Testing } \\
\text { result }\end{array}$ & Residual \\
\hline 1 & 23.33 & 2.27 & 27.85 & -1.21 & 33.91 & 1.26 & 13.28 & -3.23 \\
\hline 2 & 22.16 & 1.10 & 29.47 & 0.41 & 31.10 & -1.55 & 15.45 & -1.05 \\
\hline 3 & 22.34 & 1.28 & 28.95 & -0.11 & 32.09 & -0.56 & 14.91 & -1.60 \\
\hline 4 & 23.45 & 2.39 & 28.77 & -0.29 & 32.62 & -0.03 & 13.63 & -2.87 \\
\hline 5 & 22.95 & 1.89 & 28.08 & -0.98 & 34.12 & 1.47 & 13.27 & -3.24 \\
\hline 6 & 22.55 & 1.49 & 29.27 & 0.21 & 32.67 & 0.02 & 13.85 & -2.65 \\
\hline 7 & 22.19 & 1.13 & 29.59 & 0.53 & 31.95 & -0.70 & 14.59 & -1.91 \\
\hline 8 & 23.06 & 2.00 & 28.59 & -0.47 & 31.26 & -1.39 & 15.47 & -1.04 \\
\hline 9 & 22.59 & 1.53 & 29.21 & 0.15 & 32.12 & -0.53 & 14.40 & -2.11 \\
\hline \multirow[t]{3}{*}{10} & 22.49 & 1.43 & 28.64 & -0.42 & 33.16 & 0.51 & 13.97 & -2.54 \\
\hline & \multicolumn{8}{|c|}{ Average Values } \\
\hline & 22.71 & 1.65 & 28.84 & -0.22 & 32.50 & -0.15 & 14.28 & -2.22 \\
\hline
\end{tabular}




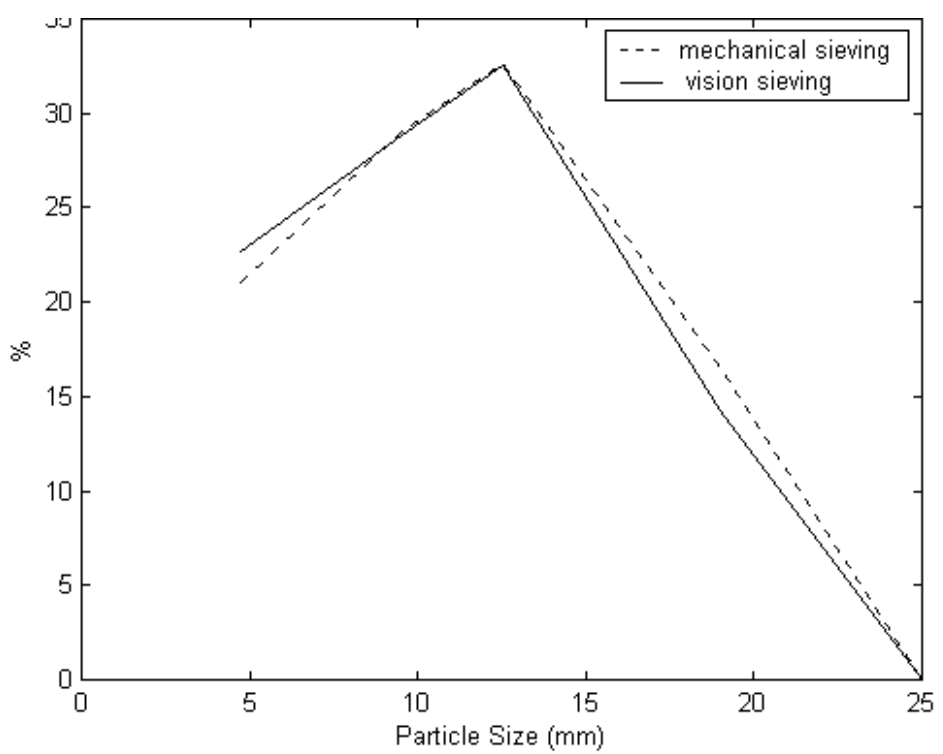

Figure 8.7 Percent Retained Correlation

Table 8.3 Percent Passing Result Comparison

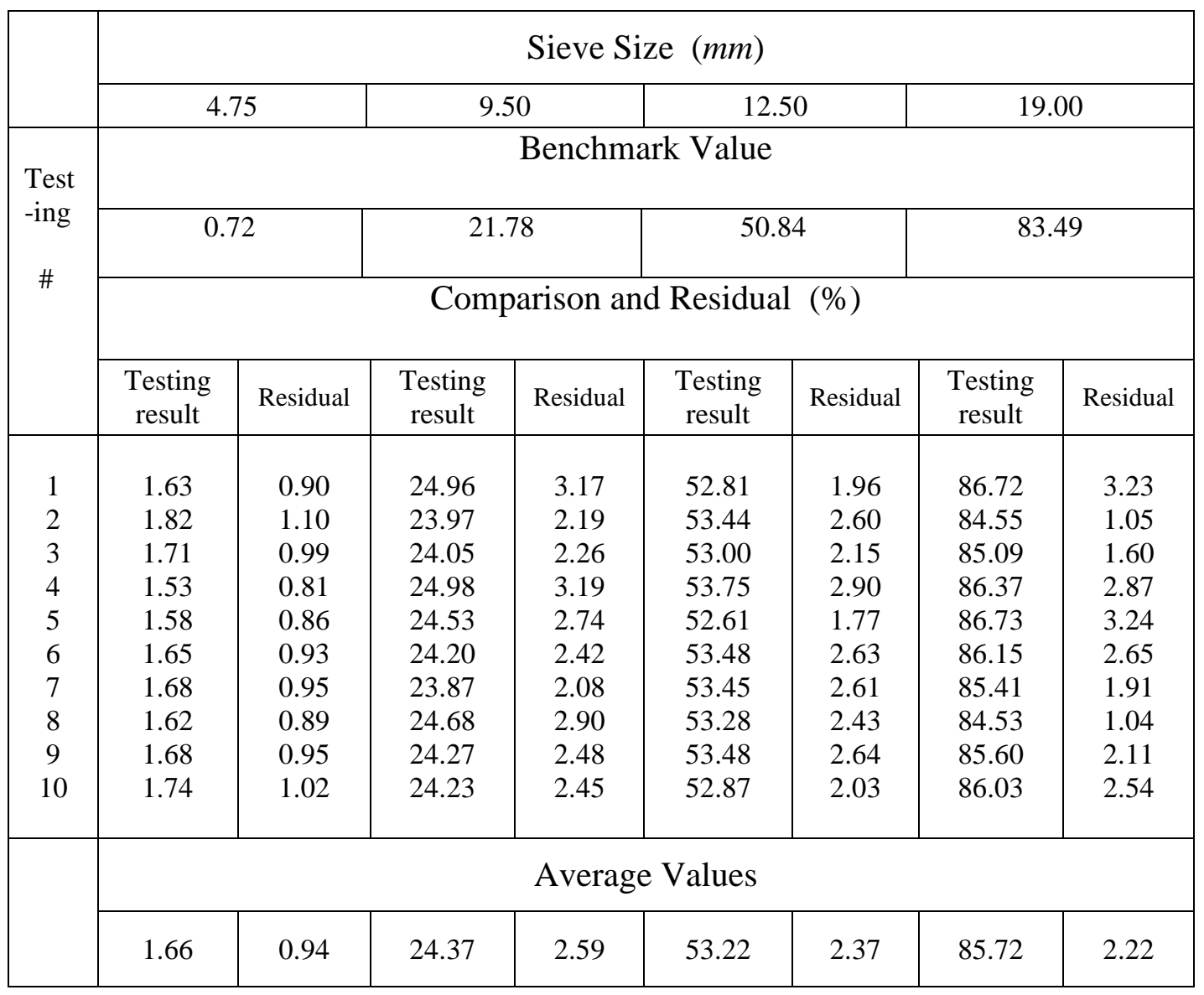




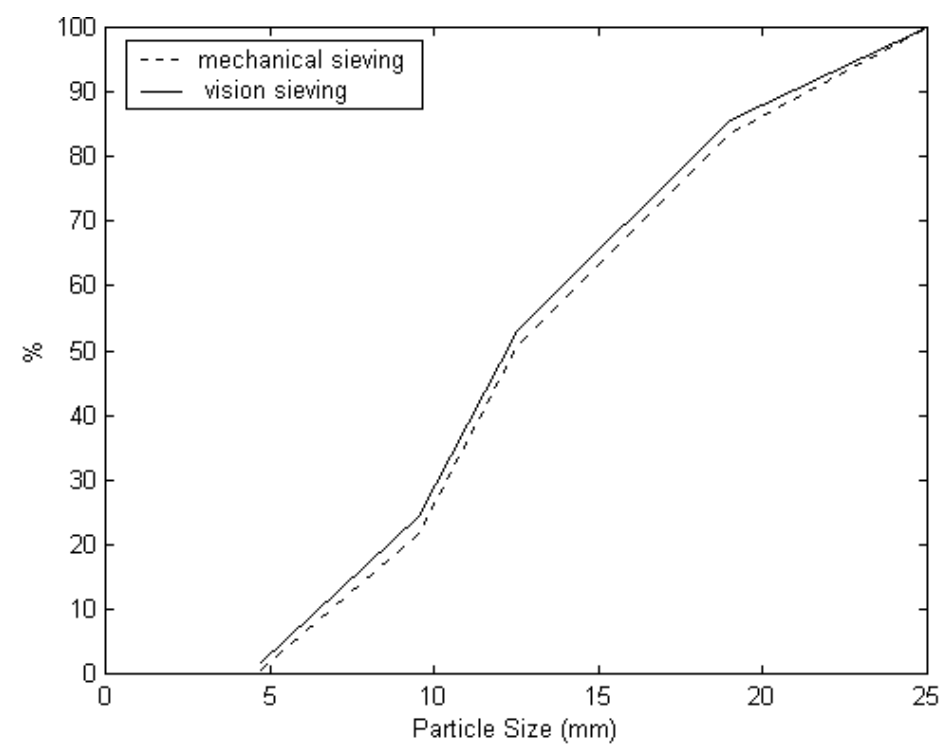

Figure 8.8 Percent Passing Correlation

The vision sieving results of percent passing are all over-estimated after the ten algorithm executions, but the residuals all are under 3 points, which is reasonably acceptable. Several literature reports concerning video grading point out that sample preparation and segmentation methods are a primary source of error. There can be numerous causes that contribute to the testing error, as stated in section 7.8. To analyze aggregates samples comprised of a mix of widely different particles, sorting the sample into groups of similar size is suggested [33, 39]. 


\section{CONCLUSIONS AND FUTURE RESEARCH}

\subsection{Conclusions}

In this dissertation, the feasibility was investigated of using a non-contact optical technique to provide information on crushed limestone aggregates' gradation. The research was conducted mainly on the following three areas and have contributed some insights to prospective application of optical sieving which is to replace relatively slower mechanical methods.

First, particles are often touching and overlapping in the imaged scene. One major contribution of this work has been the development of a simple and effective method to discriminate the touching and overlapping particles in the imaged scene.

Second, standards for classifying particles are generally based on size and mass. Mass needs to be known in order to perform gradation. A second major contribution is the development of a volume model that relates variables available from 2-D aggregate image to particle volume. Assuming constant density, mass is estimated from volume.

Third, as Superpave stipulates, percent-passing curve is used to measure proper mixture of particles of varying sizes. The conventional way of obtaining this curve is based on mechanical sieving. A contribution made in this work is that using statistical analysis, a correlation between mechanical and optical sieving has been constructed.

\subsection{Future Research}

There are three major areas that are related to this research and may need to be studied further. 
First, is there a possibility to apply the same technique to fine particles? It is believed that the processes developed in this research project constitute a feasible approach to the problem of optical sieving of fine aggregates. Although the methods in this research were only demonstrated for the coarse aggregates, it is believed that similar techniques could be applied to fine aggregates by positioning the camera closer to the particles or by using a longer focal length lens to obtain greater magnification, or simply by using a higher resolution camera. It is unlikely that any single scanning technology can yield the desired accuracy for measuring particle sizes over a very broad range. For example, one may need two different grading station designs; one optimized for scanning fine aggregates and a second optimized for scanning coarse aggregates [33]. Since fine particles and dust tend to agglomerate in piles several layers thick, some additional methods may be necessary to evaluate the fines.

Second, specifications are given to coarse aggregate angularity in the Superpave guidebook. In this work, significant effort to this subject has not been devoted. However, it is reasonable to believe that using the similar methods developed in this work for particle shape characterization, automated means to measure coarse aggregate angularity could be feasible.

Consideration of the source of system error is a broad and important topic. There are many factors inherent in the measuring system that adversely affect its accuracy. Of interest would be studies of various types of aggregates and their characteristics, requirements for camera resolution, sampling methods and limitation, and refinements of the volume and sieving models. N. Maerz has investigated some aspects of system error in [25], but the topic remains a rich one for continuing the work. 
The approach developed in this work was based on a relatively low cost vision system and sophisticated image analysis. The algorithms were tested on a limited number of samples, and have produced encouraging results. The approaches described in this dissertation are theoretically sound and practically plausible. The research shows that the automation of coarse aggregate inspection is a feasible idea. 


\section{BIBLIOGRAPHY}

1. US Department of Transportation, Federal Highway Administration, Background of Superpave Asphalt Mixture Design and Analysis. Publication No. FHWA-SA-95-003, February, 1995.

2. Rafeal G. Gonzalez, and Richard E. Woods, Digital Image Processing. (Massachusetts: Addison-Wesley Publishing, 1992).

3. Mohammed Bennamoun, and Boualem Boashash. "A Structural-Description-Based Vision System for Automatic Object Recognition." IEEE Transactions on system, man, and cybernetics-Part B: Cybernetics, vol. 27, no. 6 (1997): 893-906.

4. R. M. Parkin, D. W. Calkin, and M. R. Jackson, "Roadstone Aggregate: An Intelligent Opto-Mechatronic Product Classifier for Sizing and Grading." Mechatronics, vol. 5, no. 5 (1995): 461-467.

5. Carlo Castellini, Franco Francini, and Giuseppe Longobardi, and Enrico Pampaloni, "On-Line Characterization of the Shape and Size of Particles." Part. Part. Syst. Charact. vol. 10, no. 1 (1993): 7-10.

6. P. L. Rosin, and G. A. W. West, "Curve Segmentation and Recognition by Superellipses." IEE Proceedings-Vision, Image, Signal Processing, vol. 143, no. 3 (1995): 280-288.

7. N. N. Clark, "Three Techniques for Implementing Digital Fractal Analysis of Particle Shape.” Powder Technology, vol. 46, no. 1 (1986).

8. James Pickett, Nigel Clark, and Stephanie Shuker, "A Re-examination of Piper's Shape Analysis Technique.” Part. Part. Syst. Charact. vol. 8 (1991): 110-115. 
9. Nigel N. Clark, and Stephanie Reilly, "Template Matching for Particle Shape Description.” Particulate Science and Technology, vol. 13, no. 2 (1995): 69-83.

10. S. Reilly, and N. N. Clark, "Computer Based General Shape Description with Polygonal Harmonics and a Roughness Index.” Proc. Instn. Mech. Engrs. vol. 205 (1991): 103-111.

11. Clark N. N., and T. P. Meloy, "Delta Analysis of Particle Surface Fine Structure." Journal of Powder \& Bulk Solids Technology, vol. 9, no. 1 (1985): 1-8.

12. Anthony J. Maeder, and Nigel N. Clark, "Harmonic Endurance: A New Shape Descriptor Derived from Polygonal Harmonics.” Powder Technology, vol. 68 (1991): 137-143.

13. Thomas P. Meloy, and Nigel N. Clark, "Fourier Analysis: A Computer Based Technique for Particle Shape Characterization.” Conference on Computers in the Coal Industry, Morgantown, WV, June 1986, Proceedings, pp. 309-319.

14. H. J. Noordmans, and A. W. M. Smeulders, "Detection and Characterization of Isolated and Overlapping Spots." Computer Vision and Image Understanding, vol. 70, no. 1 (1998): 23-35.

15. Edward R.Dougherty, An Introduction to Morphological Image Processing. vol. TT9 (Washington: SPIE -The International Society for Optical Engineering, 1992).

16. William W. Hines, and Douglas C. Montgomery, Probability and Statistics in Engineering and Management Science. 2d ed., (New York: John Wiley \& Sons, Inc. 1980).

17. Douglas C. Montgomery, Design and Analysis of Experiments. (New York: John Wiley \& Sons, Inc. 1976). 
18. Raymond H. Myens, Classical and Modern Regression with Applications. 2d ed., (PWS-KENT Publishing Company, 1990).

19. Shibata Toshiharu, and Yamaguchi Kenji, "Shift X, Y-coordinate Direction of Line Figures and the Extraction of Particle Shape Information." Powder Technology, vol. 81, no. 2 (1994).

20. O.R. Mitchell, K. Fukunaga, TP. Wallace, "Three-dimensional Shape Analysis Using Local Descriptors." IEEE Trans. Pattern Analy. and Mach. Intellig., vol. 3, no. 3 (1981).

21. Luc Vincent, “Applied Morphological and Nonlinear Image Analysis Techniques." Short Course Notes, Xerox Scansoft, Palo Alto, CA, 1998.

22. S. R. Krogh, “Crushing Characteristics.” Powder Technology, vol. 27 (1980): 171181.

23. R. P. King, "Linear Stochastic Model for Mineral Liberation.” Powder Technology, vol. 81 (1994): 217-234.

24. Alexander V. Potapov, and Charles S. Campbell, "Computer Simulation of ImpactInduced Particle Breakage.” Powder Technology, vol. 87 (1996): 207-216.

25. Norbert H. Maerz, and Wei Zhou, "Optical Digital Fragmentation Measuring Systems - Inherent Sources of Error.” FRAGBLAST, The International Journal for Blasting and Fragmentation, vol. 2, no.4 (1998): 415-431.

26. N. H. Maerz, "Aggregate Sizing and Shape Determination Using Digital Image Processing." International Center for Aggregate Research (ICAR) $6^{\text {th }}$ Annual Symposium Proceedings, St. Louis, Missouri, April 19-20, 1998, pp. 195-203. 
27. N. H. Maerz, "Reconstructing 3-D Block Size Distribution from 2-D Measurements on Sections." Proceedings of the FRAGBLAST 5 Workshop on Measurement of Blast Fragmantation, Montreal, Quebec, Canada, 23-24 Aug., 1996, pp. 39-43.

28. L. Vincent, and P. Soille, "Watersheds in Digital Spaces: An Efficient Algorithm Based on Immersion Simulation.” IEEE Trans. Pattern Anal. Machine Intell., vol. 13, no. 6 (1991): :583-598.

29. George W. Snedecor, and William G. Cochran, Statistical Methods. $6^{\text {th }}$ ed., (Iowa: The Iowa State University Press, Ames, Iowa, 1967).

30. "VDG-40 Measurement of Particle Size Distribution of Granular Mixtures." Commercial Catalogue for Laboratory Central Des Ponts and Chaussees (LCPC); also in VDG 40 Technical Bulletin, Ministere de I'Equipment, July 1995.

31. R. L. Weingart, and B. D. Prowell, "Specification Development Using the VDG-40 Videograder for Shape Classification of Aggregates." Proceedings of the $7^{\text {th }}$ Annual Symposium, International Center of Aggregate Research, University of Texas, Austin, April 19-21, 1999.

32. Chetana Rao, and Erol Tutumluer, "A New Image Analysis Approach for Determination of Volume of Aggregates." Transportation Research Board, $79^{\text {th }}$ Annual Meeting, Washington, D.C , January 9-13, 2000.

33. H. Kim, C. Browne, A. Rauch, and C. Haas, "Technical Aspects of Implementing Rapid Aggregate Gradation." Proceedings of the $8^{\text {th }}$ Annual ICAR Symposium, International Center for Aggregates Research, University of Texas at Austin, April 12-14, 2000.

34. Chetana Rao, and Erol Tutumluer, "Flat and Elongated Ratio Determination Using the University of Illinois Image Analyzer." Proceedings of the $8^{\text {th }}$ Annual ICAR 
Symposium, International Center for Aggregates Research, University of Texas at Austin, April 12-14, 2000.

35. WipWare Inc. web site: http://wipware.com, July, 2000.

36. Micromeritics Corporation website: http://www.Micromeritics.com/ps_optisizer.html, July, 2000.

37. N. H. Mearz, and W. Zhou, "Flat and Elongated: Advances Using Digital Image Analysis." Proceedings of the $7^{\text {th }}$ Annual ICAR Symposium, International Center for Aggregates Research, University of Texas at Austin, April19-21, 1999.

38. N. H. Mearz, "Online Fragmentation Analysis: Achievements in the Mining Industry." Proceedings of the $7^{\text {th }}$ Annual ICAR Symposium, International Center for Aggregates Research, University of Texas at Austin, April19-21, 1999.

39. A. Rauch, C. Hass, and H. Kim, "State of the Art in Aggregate Classification." Proceedings of the $7^{\text {th }}$ Annual ICAR Symposium, International Center for Aggregates Research, University of Texas at Austin, April 19-21, 1999.

40. T. C. Palangio, and N. H. Maerz, "Case Studies Using the Wipfrag Image Analysis System." FRAGBLAST $6^{\text {th }}$ International Symposium for Rock Fragmentation By Blasting, Johannesburg, South Africa, August 8-12, 1999, pp. 117-120.

41. B. M. K. Bocoum, "Hatch Sieving of Cylindrical Particles Cascadography." (master's thesis, West Virginia University, n.d.). 


\title{
APPENDIX I
}

\section{Modeling Sample Statistics (Total of 501 particles)}

\author{
Area: Projected area $\left(\mathrm{mm}^{2}\right)$ \\ Aps. : Aspect ratio \\ $D_{m a j}:$ Major diameter $(\mathrm{mm})$ \\ $D_{\text {min }}$ : Minor diameter $(\mathrm{mm})$ \\ $M N_{\text {sig }}$ : Non-normalized signature mean $(\mathrm{mm})$ \\ $V R_{\text {sig }}$ : Non-normalized signature variance $\left(\mathrm{mm}^{2}\right)$ \\ Mass: Particle actual mass $(g)$
}

\begin{tabular}{|c|c|c|c|c|c|c|}
\hline Area & Asp. & $D_{m a j}$ & $D_{\min }$ & $M N_{s i g}$ & $V R_{\text {sig }}$ & Mass \\
\hline 79.7623 & 1.0797 & 9.0093 & 8.3439 & 4.3932 & 0.3751 & 0.4110 \\
\hline 43.6570 & 2.1019 & 8.8433 & 4.2073 & 3.1808 & 0.9354 & 0.2040 \\
\hline 50.7597 & 1.0618 & 6.8671 & 6.4672 & 3.3289 & 0.2773 & 0.2940 \\
\hline 63.7812 & 1.2011 & 8.7778 & 7.3084 & 3.8873 & 0.3601 & 0.3900 \\
\hline 37.1462 & 1.2040 & 5.5286 & 4.5919 & 2.6133 & 0.1777 & 0.2640 \\
\hline 50.1678 & 1.4524 & 8.0294 & 5.5286 & 3.4113 & 0.4535 & 0.2600 \\
\hline 44.8408 & 1.2620 & 7.4402 & 5.8956 & 3.0841 & 0.2023 & 0.2780 \\
\hline 53.1272 & 2.1019 & 8.8433 & 4.2073 & 3.5668 & 0.9646 & 0.2720 \\
\hline 33.0030 & 1.0697 & 4.9120 & 4.5919 & 2.4312 & 0.1575 & 0.2420 \\
\hline 22.3490 & 2.5000 & 3.2755 & 1.3102 & 1.7460 & 0.2320 & 0.2360 \\
\hline 36.5543 & 1.3173 & 5.7814 & 4.3889 & 2.6613 & 0.1340 & 0.3060 \\
\hline 31.2273 & 2.4512 & 6.4672 & 2.6384 & 2.4935 & 0.7110 & 0.2220 \\
\hline 56.0867 & 2.9265 & 10.8826 & 3.7186 & 3.8627 & 1.8787 & 0.6000 \\
\hline 31.8192 & 2.0487 & 6.4672 & 3.1568 & 2.5225 & 0.5574 & 0.1640 \\
\hline 45.4327 & 1.4717 & 7.7661 & 5.2769 & 3.1783 & 0.3998 & 0.1650 \\
\hline 37.1462 & 1.4626 & 6.2992 & 4.3068 & 2.7250 & 0.3054 & 0.1880 \\
\hline 57.2705 & 1.1776 & 7.4180 & 6.2992 & 3.5148 & 0.2784 & 0.3100 \\
\hline 49.5759 & 1.2620 & 7.4402 & 5.8956 & 3.3514 & 0.3253 & 0.3820 \\
\hline 62.5975 & 1.6759 & 8.8433 & 5.2769 & 3.9591 & 0.8836 & 0.4890 \\
\hline 36.5543 & 1.4626 & 6.2992 & 4.3068 & 2.7048 & 0.3180 & 0.2820 \\
\hline 40.6975 & 2.5546 & 8.0644 & 3.1568 & 3.0127 & 0.7156 & 0.2510 \\
\hline 44.2489 & 2.1019 & 8.8433 & 4.2073 & 3.1931 & 0.7903 & 0.1920 \\
\hline 69.7001 & 1.3119 & 8.8990 & 6.7834 & 4.1040 & 0.3806 & 0.4270 \\
\hline 76.8028 & 1.8238 & 11.5149 & 6.3136 & 4.4950 & 1.3156 & 0.5300 \\
\hline 74.4353 & 1.0000 & 8.0294 & 8.0294 & 4.1791 & 0.2462 & 0.3160 \\
\hline 41.2894 & 1.9585 & 7.3573 & 3.7567 & 2.9718 & 0.6607 & 0.2520 \\
\hline 48.9840 & 1.1667 & 7.6806 & 6.5834 & 3.2703 & 0.2611 & 0.4100 \\
\hline 36.5543 & 1.2040 & 5.5286 & 4.5919 & 2.6008 & 0.1789 & 0.3190 \\
\hline 60.8218 & 1.2184 & 7.6747 & 6.2992 & 3.7048 & 0.4201 & 0.4940 \\
\hline 67.3326 & 2.3898 & 11.7388 & 4.9120 & 4.2317 & 1.6890 & 0.5170 \\
\hline 47.8002 & 1.4000 & 7.6806 & 5.4861 & 3.2664 & 0.3090 & 0.4040 \\
\hline 51.3516 & 1.2287 & 8.0888 & 6.5834 & 3.3832 & 0.3439 & 0.3460 \\
\hline 57.2705 & 1.3723 & 8.5279 & 6.2145 & 3.6654 & 0.5241 & 0.3990 \\
\hline 50.7597 & 2.6974 & 10.0305 & 3.7186 & 3.6012 & 1.5517 & 0.3100 \\
\hline 47.8002 & 1.6245 & 8.5723 & 5.2769 & 3.3323 & 0.7259 & 0.3520 \\
\hline 50.7597 & 1.7544 & 8.0559 & 4.5919 & 3.2774 & 0.5542 & 0.2630 \\
\hline 33.5949 & 1.5000 & 6.5834 & 4.3889 & 2.5218 & 0.1847 & 0.2130 \\
\hline 41.8813 & 2.8190 & 8.8990 & 3.1568 & 3.2486 & 1.4370 & 0.3920 \\
\hline 70.2920 & 1.6189 & 10.2212 & 6.3136 & 4.2535 & 0.7799 & 0.3850 \\
\hline 54.3110 & 1.3723 & 8.5279 & 6.2145 & 3.4875 & 0.3252 & 0.3290 \\
\hline 40.6975 & 2.1593 & 8.0294 & 3.7186 & 3.0389 & 0.9985 & 0.2570 \\
\hline 47.8002 & 1.6759 & 8.8433 & 5.2769 & 3.3514 & 0.6560 & 0.3140 \\
\hline 52.5353 & 1.0769 & 6.7834 & 6.2992 & 3.3669 & 0.1805 & 0.3740 \\
\hline 37.7381 & 1.4626 & 6.2992 & 4.3068 & 2.8003 & 0.3753 & 0.2700 \\
\hline 46.6164 & 1.6196 & 7.4372 & 4.5919 & 3.0853 & 0.3686 & 0.2120 \\
\hline 43.6570 & 1.1204 & 6.3136 & 5.6350 & 3.0026 & 0.2523 & 0.3320 \\
\hline
\end{tabular}




\begin{tabular}{|c|c|c|c|c|c|c|}
\hline .0030 & 1.2174 & 5.1220 & 4.2073 & 2.4590 & 0.1752 & \\
\hline & & & & & & \\
\hline .0651 & 1.0000 & 6.2992 & 6.2992 & & & \\
\hline 3.0651 & 1.0000 & 6.2992 & 6.2992 & & & \\
\hline 6.0246 & 1.4744 & 8.0888 & .4861 & & & \\
\hline & 1.4047 & 5.2769 & .7567 & 2.2732 & & 3060 \\
\hline & 2.2686 & 9.7704 & 4.3068 & 3.6921 & & 5570 \\
\hline & 1.2476 & 6.5834 & 5.2769 & 2.9295 & & \\
\hline & 1.5854 & & & & & \\
\hline & 1.4633 & 5.4413 & 3.7186 & 2.4596 & 2747 & \\
\hline & 1.2040 & & & & & \\
\hline .5543 & 1.0000 & 5.4413 & 5.4413 & 2.6446 & & \\
\hline & 3.4473 & & & & & \\
\hline & 1.3344 & & 6.6955 & 3.7582 & & \\
\hline & & & & & & \\
\hline & & & & & & \\
\hline & 2.4697 & 9.1838 & & 3.4110 & & \\
\hline & 2.6033 & 12.3273 & & & & \\
\hline 26.4922 & 1.3333 & 4.3889 & 3.2917 & 2.1192 & & \\
\hline & & & & & & \\
\hline & 1.4582 & 7.1625 & 4.9120 & 100 & & \\
\hline & & & & & & \\
\hline & 1.0709 & 3.1568 & 2.9478 & 1.5 & & \\
\hline & & & & & & \\
\hline 75 & 1.0000 & & 5.4413 & 2.8 & & \\
\hline & & & & & & \\
\hline & 2.0420 & 305 & & & & \\
\hline & & & & & & \\
\hline & & & & & & \\
\hline & 1.5296 & 7.5134 & 4.912 & & & \\
\hline & & & & & & \\
\hline & 1.4626 & 992 & 4.3068 & 2.7 & & \\
\hline & & & & & & \\
\hline 32 & & 084 & 5.2769 & & & \\
\hline & 1.0000 & & & & & \\
\hline 01 & 1.2922 & & & & & \\
\hline & & & & & & \\
\hline 52.5 & 1.4524 & 8.0294 & 5.5286 & & & \\
\hline & & & & & & \\
\hline & 2.19 & & & & & \\
\hline & & & & & & \\
\hline & & & & & & \\
\hline & & & & & & \\
\hline & & & & & & \\
\hline & & & & & & \\
\hline & & & & & & \\
\hline & 2.8774 & 12.3923 & & & & \\
\hline 218 & 1.5982 & 8.6963 & & 759 & & \\
\hline 60.8218 & 1.0000 & & & 3.7306 & & \\
\hline 53.7191 & 1.6616 & 8.1619 & & 038 & & \\
\hline & 1.0000 & & & & & \\
\hline & 1.5546 & & 4.3068 & 3.0 & & \\
\hline & 1.5217 & 10.4496 & & & & \\
\hline & & & & & & \\
\hline & & & & & & \\
\hline & & & & $2.5 \mathrm{C}$ & & \\
\hline & & & & & & \\
\hline & 1.8697 & & 4.9120 & 3.70 & & \\
\hline & & & & & & \\
\hline & 1.0000 & & & 3.7840 & & \\
\hline 47.2083 & 2.0663 & 8.8990 & 4.3068 & 3.3933 & & 650 \\
\hline & 2.3931 & & & & & \\
\hline & 1.6631 & 7.1625 & 4.3068 & 3.0881 & 0.3955 & 0.3050 \\
\hline & & & & & & \\
\hline & & & 6.9779 & 3.6144 & & 0.3510 \\
\hline & & & & & & \\
\hline & 1.9261 & 7.1625 & 3.7186 & 3.0241 & & 0.3260 \\
\hline & 1.4524 & 8.0294 & 5.5286 & 3.5507 & & $0.36 / 0$ \\
\hline & 1.0357 & 7.4180 & 7.1625 & 3.6906 & & 0.3200 \\
\hline 54.9029 & 2.0663 & 8.8990 & 4.3068 & 3.6390 & 0.9993 & 0.3190 \\
\hline
\end{tabular}




\begin{tabular}{|c|c|c|c|c|c|c|}
\hline & & & & & & \\
\hline & & & & & & \\
\hline & & & & & & \\
\hline & & & & & & \\
\hline & & & & & & \\
\hline & & & & & & \\
\hline & & & & & & \\
\hline & & & & & & \\
\hline & & & & & & \\
\hline & & & & & & \\
\hline & & & & & & \\
\hline & & & & & & \\
\hline & 2.43 & & & & & \\
\hline & & & & & & \\
\hline & & & & & & \\
\hline & & & & & & \\
\hline & & & & & & \\
\hline & & & & & & \\
\hline & & & & & & \\
\hline & & & & & & \\
\hline & & & & & & \\
\hline & & & & & & \\
\hline & & & & & & \\
\hline & & & & & & \\
\hline & & & & & & \\
\hline & & & & & & \\
\hline & & & & & & \\
\hline & & & & & & \\
\hline & & & & & & \\
\hline & & & & & & \\
\hline & & & & & & \\
\hline & & & & & & \\
\hline & & & & & & \\
\hline & & & & & & \\
\hline & & & & & & \\
\hline & & & & & & \\
\hline & & & & & & \\
\hline & & & & & & \\
\hline & & & & & & \\
\hline & & & & & & \\
\hline & & & & & & \\
\hline & & & & & & \\
\hline & & & & & & \\
\hline & & & & & & \\
\hline & & & & & & \\
\hline & & & & & & \\
\hline & & & & & & \\
\hline & & & & & & \\
\hline & & & & & & \\
\hline & & & & & & \\
\hline & & & & & & \\
\hline & & & & & & \\
\hline & & & & & & \\
\hline & & & & & & \\
\hline & 000 & & 12 & 354 & 0.3968 & \\
\hline & & & & & & \\
\hline & & & & & & \\
\hline & & & & & & \\
\hline & & & & & & \\
\hline & & & & & & \\
\hline & & & & & & \\
\hline & & & & & & \\
\hline & 1.9680 & & & & 1.5020 & \\
\hline & & & & & & \\
\hline & & & 10.4496 & 6.8799 & & \\
\hline & & & & & & \\
\hline & 1.0034 & & 9.1838 & 4.7871 & 0.4857 & \\
\hline & & 15.0692 & & 5.5192 & & \\
\hline & & & & & & \\
\hline 56.0867 & 1.0000 & 7.1625 & 7.1625 & 3.5215 & 0.1204 & 0.4120 \\
\hline
\end{tabular}




\begin{tabular}{|c|c|c|c|c|c|c|}
\hline & 1.2605 & & & & & \\
\hline & & & & & & \\
\hline & .3237 & & & & & \\
\hline & & & & & & \\
\hline 72.0677 & .0033 & & & & & \\
\hline & 2.4466 & & & & & \\
\hline & & & & & & \\
\hline 7.8296 & 2.5211 & & 7.3107 & 6.4370 & & 1.0570 \\
\hline & & & & & & \\
\hline & 1.0000 & & & & & \\
\hline & & & & & & \\
\hline & & & 4.5 & & & \\
\hline & & & & & & \\
\hline & 2.4849 & 13. & 5.4413 & & & \\
\hline & & & & & & \\
\hline & & & & & & \\
\hline & & & & & & \\
\hline & & & & & & \\
\hline 94 & 2.8 & & & & & \\
\hline & & & & & & \\
\hline & 2.0 & & & & & \\
\hline & & & & & & \\
\hline & 1.3 & & & & & \\
\hline & & & & & & \\
\hline & 2.3 & & & & & \\
\hline & & & & & & \\
\hline & & & 6.25 & & & \\
\hline & & & & & & \\
\hline & & & & & & \\
\hline & & & & & & \\
\hline & & & & & & \\
\hline & & & & & & \\
\hline & & & & & & \\
\hline & & & & & & \\
\hline & & & & & & \\
\hline & & & & & & \\
\hline & & & & & & \\
\hline & & & & & & \\
\hline & & & & & & \\
\hline & & & & & & \\
\hline & & & & & & \\
\hline & & & & & & \\
\hline & & & & & & \\
\hline & & & & & & \\
\hline & & & & & & \\
\hline & & & & & & \\
\hline & & & & & & \\
\hline & & & & & & \\
\hline & & & & & & \\
\hline & & & & & & \\
\hline 520 & & & & & & 6090 \\
\hline & & & & & & \\
\hline & & & & & & \\
\hline & & & & & & \\
\hline 93 & & & & & & \\
\hline & & & & & & \\
\hline & & & 10.2 & & & \\
\hline & & & & & & 100 \\
\hline & & & & & & \\
\hline & & & & & & \\
\hline 56.1160 & & & 12.9345 & 520 & & 1.6040 \\
\hline & & & & & & \\
\hline 105.2135 & 1.7631 & & 7.9153 & 5.4010 & 2.0249 & 1.1520 \\
\hline & & & & & & \\
\hline 182.7511 & & & 12.0989 & & & 3.8620 \\
\hline & & & & & & \\
\hline 86.8649 & & & 7.1625 & 4.5482 & 0.5018 & 0.8300 \\
\hline 57.2998 & & & & & & \\
\hline & & & 6.2992 & & & 0.6260 \\
\hline 86.86 & 1.7093 & 10.7 & & & & 0.6360 \\
\hline
\end{tabular}




\begin{tabular}{|c|c|c|c|c|c|c|}
\hline 72.6596 & 1.3806 & 8.6963 & 6.2992 & 4.1058 & 0.4990 & \\
\hline 108.1730 & 1.0000 & 11.7388 & 11.7388 & 5.2987 & 0.4603 & 1.2190 \\
\hline 83.3136 & 1.1477 & 9.2154 & 8.0294 & 4.4895 & 0.4281 & 0.6760 \\
\hline 64.9650 & 1.4546 & 9.1838 & 6.3136 & 4.0058 & 0.8988 & 0.6020 \\
\hline 122.9702 & 1.9311 & 14.3249 & 7.4180 & 5.9500 & 2.3281 & 1.2670 \\
\hline 151.9728 & 1.4082 & 14.7147 & 10.4496 & 6.4686 & 1.3254 & 1.6760 \\
\hline 89.8244 & 1.1535 & 10.5942 & 9.1840 & 4.8459 & 0.8047 & 0.8080 \\
\hline 88.0487 & 1.1350 & 9.4705 & 8.3439 & 4.6632 & 0.2890 & 0.9320 \\
\hline 75.0271 & 1.0000 & 8.0294 & 8.0294 & 4.2545 & 0.3175 & 0.3860 \\
\hline 135.3999 & 1.2171 & 12.8947 & 10.5942 & 6.1248 & 0.9791 & 1.2350 \\
\hline 73.8434 & 1.8075 & 10.4496 & 5.7814 & 4.3545 & 1.0245 & 0.3710 \\
\hline 106.9892 & 2.7984 & 15.4710 & 5.5286 & 5.6280 & 3.2864 & 0.8800 \\
\hline 103.4379 & 1.5116 & 13.8828 & 9.1840 & 5.3700 & 2.0040 & 1.3090 \\
\hline 55.4948 & 1.6428 & 8.4147 & 5.1220 & 3.6611 & 0.7764 & 0.5750 \\
\hline 112.9081 & 1.4087 & 12.5857 & 8.9341 & 5.4463 & 0.6789 & 1.4170 \\
\hline 147.2377 & 1.2629 & 13.9559 & 11.0504 & 6.3830 & 1.1420 & 0.8820 \\
\hline 104.0297 & 1.1724 & 10.7670 & 9.1838 & 5.1168 & 0.6026 & 1.0210 \\
\hline 133.0323 & 1.3361 & 13.6872 & 10.2441 & 5.9262 & 1.0655 & 1.0970 \\
\hline 135.3999 & 1.3689 & 15.5176 & 11.3361 & 6.0934 & 1.3459 & 1.0030 \\
\hline 125.9297 & 1.8997 & 15.3661 & 8.0888 & 5.8985 & 1.8637 & 0.4500 \\
\hline 94.5595 & 1.3938 & 9.9829 & 7.1625 & 4.8026 & 0.8350 & 1.1420 \\
\hline 44.8408 & 1.4582 & 7.1625 & 4.9120 & 3.1474 & 0.4339 & 0.5360 \\
\hline 111.7243 & 1.3075 & 11.5628 & 8.8433 & 5.3552 & 0.4970 & 1.3400 \\
\hline 146.0539 & 2.0132 & 17.3411 & 8.6136 & 6.5336 & 3.0532 & 1.1470 \\
\hline 143.0945 & 1.3097 & 14.6168 & 11.1603 & 6.2917 & 1.3589 & 1.5340 \\
\hline 280.4129 & 1.4872 & 19.7317 & 13.2678 & 8.7393 & 1.7457 & 3.0610 \\
\hline 304.6804 & 1.2095 & 20.3264 & 16.8063 & 9.4210 & 1.5487 & 4.1600 \\
\hline 262.0643 & 1.1998 & 18.2270 & 15.1911 & 8.4743 & 0.8011 & 5.0950 \\
\hline 256.1454 & 1.5802 & 19.7183 & 12.4785 & 8.4284 & 2.3970 & 4.3470 \\
\hline 291.0669 & 1.5797 & 24.2664 & 15.3612 & 9.4516 & 4.3773 & 5.4410 \\
\hline 193.9970 & 1.0000 & 16.7750 & 16.7750 & 7.3451 & 168 & 1.9110 \\
\hline 228.3266 & 2.4813 & 24.8890 & 10.0305 & 8.4393 & 7.7350 & 1.9320 \\
\hline 138.9512 & 1.4337 & 14.0082 & 9.7704 & 6.0090 & 0.7346 & 1.9450 \\
\hline 295.8020 & 1.4380 & 19.0793 & 13.2678 & 9.0719 & 2.6343 & 3.5680 \\
\hline 416.5476 & 1.5943 & 27.3336 & 17.1445 & 11.1546 & 4.3778 & 6.3480 \\
\hline 299.9453 & & 22.3429 & & & & \\
\hline 279.8210 & 1.3292 & 19.9659 & 15.0207 & 8.8602 & 1.4852 & 3.4930 \\
\hline 199.3240 & 1.3636 & 17.9545 & 13.1667 & 7.5776 & 1.4872 & 3.0340 \\
\hline 339.6019 & 1.0000 & 19.5409 & 19.5409 & 9.8057 & 0.7566 & 4.9470 \\
\hline 183.3430 & 2.0245 & 18.5929 & 9.1838 & 7.2541 & 3.4912 & 1.8700 \\
\hline 156.1160 & 1.6469 & 16.1786 & 9.8240 & 6.6206 & 1.6480 & 3.1620 \\
\hline 231.8779 & 1.2636 & 19.2795 & 15.2575 & 8.1593 & 1.5376 & 4.2230 \\
\hline 200.5078 & 2.0375 & 20.3398 & 9.9829 & 7.7205 & 3.5845 & 2.8950 \\
\hline 263.8400 & 1.5252 & 20.6226 & 13.5210 & 8.8221 & 2.8419 & 2.8310 \\
\hline 299.9453 & 1.2684 & 21.1665 & 16.6879 & 9.2899 & 1.9715 & 3.8300 \\
\hline 147.8296 & 1.8638 & 16.5857 & 8.8990 & 6.3042 & 2.4060 & 1.5020 \\
\hline 176.8322 & 1.2881 & 14.8360 & & 6.8609 & & 1.7210 \\
\hline 128.2972 & 1.0647 & 12.0695 & 11.3361 & 5.9320 & 0.7837 & 1.4950 \\
\hline 186.3024 & & 15.1698 & & 7.0951 & & 1.3030 \\
\hline 143.6864 & 1.4582 & 14.3249 & 9.8240 & 6.3319 & 1.2873 & 1.0850 \\
\hline 143.0945 & 1.1478 & 13.2197 & 11.5174 & 6.1067 & 0.6325 & 1.1470 \\
\hline 325.3965 & 1.0940 & 20.5308 & 18.7673 & 9.7433 & 1.1664 & 5.6810 \\
\hline 214.1212 & 1.4107 & 18.6006 & 13.1852 & 7.8445 & 1.5786 & 1.9300 \\
\hline 214.1212 & 1.6435 & 18.5306 & 11.2750 & 7.8287 & 1.9900 & 3.1500 \\
\hline 173.8727 & 1.6667 & 16.4584 & 9.8750 & 7.1676 & 2.4274 & 2.2430 \\
\hline 159.6674 & 2.4812 & 19.9000 & 8.0202 & 7.1265 & 5.5197 & 1.6130 \\
\hline 398.7909 & 1.8503 & 29.0713 & 15.7114 & 11.1853 & 6.0729 & 9.0520 \\
\hline 230.1023 & 1.0239 & 16.8875 & 16.4939 & 8.0816 & 1.0446 & 4.7610 \\
\hline 167.9538 & 1.2941 & 16.3346 & 12.6220 & 6.8399 & 1.5287 & 1.3050 \\
\hline 268.5751 & 1.2373 & 18.5929 & 15.0267 & 8.8129 & 2.2491 & 2.2800 \\
\hline 127.1135 & 1.0593 & 11.2750 & 10.6434 & 5.7089 & 0.2168 & 1.7100 \\
\hline 124.1540 & 1.7636 & 15.1911 & 8.6136 & 5.9472 & 2.1015 & 0.8450 \\
\hline 176.2403 & 1.8642 & 17.1795 & 9.2154 & 7.1428 & 2.7149 & 2.5200 \\
\hline 245.4914 & 1.1267 & 16.2913 & 14.4596 & 8.4939 & 2.2017 & 4.0500 \\
\hline 289.2912 & 2.5732 & 25.1415 & 9.7704 & 9.3025 & 8.6492 & 3.7100 \\
\hline 284.5561 & 1.0329 & 17.3927 & 16.8389 & 8.9552 & 1.1641 & 2.2700 \\
\hline 286.3318 & 1.0326 & 18.7847 & 18.1915 & 9.0178 & 0.7478 & 3.8030 \\
\hline 237.2049 & 1.7153 & 21.2568 & 12.3923 & 8.2121 & 3.9021 & 2.9000 \\
\hline 240.7563 & 1.2500 & 19.3971 & 15.5176 & 8.3865 & 2.3694 & 4.3000 \\
\hline 333.0911 & 1.2251 & 19.1725 & 15.6503 & 9.7797 & 1.6583 & 7.6170 \\
\hline
\end{tabular}




\begin{tabular}{|c|c|c|c|c|c|c|}
\hline & & & & & & \\
\hline & & & & & & \\
\hline & 1.5853 & & & & & \\
\hline & 1.6755 & & & & & \\
\hline & 1.2042 & & & & & \\
\hline & 1.8160 & & & & & \\
\hline & & & & & & \\
\hline 7753 & 1.0000 & & 18.4793 & & & \\
\hline & & & & & & \\
\hline & 1.7000 & 18.6 & & & & \\
\hline & & & & & & \\
\hline & 1.0000 & & & & & \\
\hline & & & & & & \\
\hline & & 22. & & & & \\
\hline & & & & & & \\
\hline & & & & & & \\
\hline & 1.5265 & & & & & \\
\hline & & & & & & \\
\hline 63 & 2.2949 & & & & & \\
\hline & & & & & & \\
\hline & & & & & & \\
\hline & & & & & & \\
\hline & 1.0 & & & & & \\
\hline & & & & & & \\
\hline & 1.40 & 22. & & & & \\
\hline & & & & & & \\
\hline & 1.45 & & & & & \\
\hline & & & & & & \\
\hline & & & & & & \\
\hline & & & & & & \\
\hline & & & & & & \\
\hline & 1.3 & & & & & \\
\hline & & & & & & \\
\hline & & & & & & \\
\hline & & & & & & \\
\hline & & & & & & \\
\hline & & & & & & \\
\hline & & & & & & \\
\hline & & & & & & \\
\hline & 1.3 & & & & & \\
\hline & & & & & & \\
\hline & & & & & & \\
\hline & & & & & & \\
\hline & & & & & & \\
\hline & & & & & & \\
\hline & & & & & & \\
\hline & & & & & & \\
\hline & & & & & & \\
\hline & & & & & & \\
\hline & & & & & & 250 \\
\hline & & & & & & \\
\hline & 1.25 & & & & & \\
\hline & & & & & & \\
\hline & & & & & & \\
\hline & & & & & & \\
\hline & 1.45 & & & & & \\
\hline & & & & & & \\
\hline & 1.0145 & & 15. & & & \\
\hline & & & & & & \\
\hline & & & & & & \\
\hline & 2.2173 & & & & & \\
\hline & & & & & & \\
\hline & 1.0000 & & & & 0.16 & \\
\hline & & & & & & \\
\hline & 1.1639 & 16.8293 & & & 1.7898 & 1.7980 \\
\hline & & & & & & \\
\hline & 1.7426 & 17.4054 & & & 2.5224 & 2.4000 \\
\hline & 1.4296 & & 10.5114 & & & \\
\hline & 1.5023 & 23.6029 & 15.7114 & & 3.05 & 3.302 \\
\hline & 1.66 & & & 6.2074 & & 1.1530 \\
\hline
\end{tabular}




\begin{tabular}{|c|c|c|c|c|c|c|}
\hline 233.6536 & 1.6942 & 20.1643 & 11.9022 & 8.0649 & 2.8011 & 14.6500 \\
\hline 273.3102 & 1.5766 & 21.2222 & 13.4605 & 8.7381 & 2.4405 & 5.5050 \\
\hline 163.8106 & 2.3838 & 17.9100 & 7.5134 & 6.9738 & 4.8760 & 2.0500 \\
\hline 314.7425 & 1.0439 & 18.4271 & 17.6527 & 9.3812 & 1.0481 & 4.7070 \\
\hline 581.09 & 1.49 & 31.75 & 21.28 & 13.27 & 5.59 & 10.7100 \\
\hline 544.40 & 2.04 & 32.89 & 16.11 & 13.11 & 11.85 & 11.8720 \\
\hline 452.06 & 1.00 & 22.16 & 22.16 & 11.44 & 0.89 & 10.5750 \\
\hline 465.67 & 1.37 & 26.08 & 19.03 & 11.85 & 4.06 & 13.1580 \\
\hline 556.83 & 1.64 & 30.59 & 18.67 & 12.69 & 6.37 & 12.0200 \\
\hline 572.81 & 1.26 & 29.04 & 22.97 & 12.89 & 3.22 & 18.1270 \\
\hline 558.60 & 1.02 & 26.09 & 25.66 & 12.80 & 1.47 & 13.8900 \\
\hline 669.28 & 1.36 & 30.31 & 22.25 & 13.93 & 4.96 & 12.0110 \\
\hline 748.60 & 1.41 & 32.21 & 22.92 & 14.74 & 5.74 & 10.1970 \\
\hline 597.67 & 1.19 & 26.26 & 22.04 & 13.10 & 2.26 & 9.5780 \\
\hline 427.20 & 1.89 & 29.43 & 15.53 & 11.33 & 7.59 & 8.0010 \\
\hline 688.22 & 1.40 & 33.77 & 24.20 & 14.33 & 6.45 & 16.2100 \\
\hline 587.01 & 2.04 & 36.54 & 17.95 & 14.30 & 20.35 & 15.9500 \\
\hline 674.61 & 1.00 & 29.07 & 29.07 & 14.16 & 2.50 & 14.8450 \\
\hline 676.98 & 1.48 & 35.64 & 24.11 & 14.68 & 11.41 & 14.9550 \\
\hline 784.70 & 1.33 & 34.42 & 25.80 & 15.48 & 4.87 & 16.1830 \\
\hline 532.56 & 1.31 & 27.40 & 20.91 & 12.43 & 2.72 & 12.7140 \\
\hline 809.56 & 1.99 & 43.73 & 21.93 & 16.51 & 19.12 & 18.6390 \\
\hline 399.38 & 1.00 & 23.99 & 23.99 & 10.83 & 3.80 & 13.5690 \\
\hline 389.91 & 1.07 & 21.74 & 20.24 & 10.66 & 1.69 & 7.1320 \\
\hline 483.43 & 1.15 & 25.95 & 22.51 & 12.08 & 4.09 & 14.3340 \\
\hline 509.47 & 1.00 & 25.72 & 25.72 & 12.37 & 2.79 & 14.1000 \\
\hline 472.78 & 1.00 & 26.20 & 26.20 & 11.87 & 2.15 & 10.3300 \\
\hline 669.28 & 1.21 & 29.04 & 24.09 & 13.93 & 2.29 & 11.1010 \\
\hline 548.54 & 1.40 & 29.81 & 21.22 & 12.64 & 5.12 & 13.8000 \\
\hline 072.36 & 2.10 & 46.99 & 22.35 & 17.98 & 20.13 & 26.2380 \\
\hline 682.31 & 1.00 & 28.39 & 28.29 & 14.18 & 1.58 & 16.4900 \\
\hline 637.91 & 1.03 & 24.64 & 23.91 & 13.68 & 2.82 & 14.3610 \\
\hline 671.06 & 1.34 & 37.31 & 27.83 & & 6.17 & 16.3100 \\
\hline 630.22 & 1.66 & 31.31 & 18.90 & 13.55 & 6.84 & 18.1430 \\
\hline 631.40 & 1.68 & 34.11 & 20.29 & 13.56 & 7.21 & 16.2000 \\
\hline 367.42 & 1.29 & 23.02 & 17.91 & 10.41 & 3.00 & 8.9150 \\
\hline 620.16 & 1.47 & 31.39 & 21.38 & 13.53 & 6.67 & 16.2880 \\
\hline 524.27 & 1.03 & 27.33 & 26.53 & 12.55 & 2.64 & 6.2300 \\
\hline 515.99 & 1.19 & 25.79 & 21.62 & 12.21 & 2.26 & 11.0210 \\
\hline 547.95 & 1.35 & 29.50 & 21.93 & 13.07 & 6.69 & 15.2630 \\
\hline 533.74 & 1.69 & 31.93 & 18.90 & 12.80 & 8.01 & 12.2820 \\
\hline 852.77 & 2.25 & 45.79 & 20.38 & 17.03 & 23.80 & 23.1000 \\
\hline 467.45 & 1.02 & 24.18 & 23.60 & 11.85 & 4.15 & 12.1200 \\
\hline 507.70 & 1.04 & & 22.16 & 12.19 & 2.84 & 13.0680 \\
\hline 534.33 & 1.05 & 24.38 & 23.22 & 12.44 & 1.01 & 8.0100 \\
\hline 382.81 & 1.82 & & 14.30 & 11.20 & 8.37 & 9.0010 \\
\hline 542.62 & 1.33 & 31.42 & 23.60 & 12.83 & 4.63 & 16.5500 \\
\hline 727.29 & 1.38 & 34.11 & 24.68 & 14.45 & 3.89 & 10.5530 \\
\hline 605.95 & 1.22 & 28.55 & 23.48 & 13.26 & 2.10 & 13.7730 \\
\hline 640.28 & 1.20 & 27.57 & 22.92 & 13.60 & 2.04 & 10.2980 \\
\hline 427.79 & 1.00 & 23.30 & 23.30 & 11.29 & 2.96 & 11.4800 \\
\hline 436.67 & 1.00 & 20.41 & 20.41 & 11.22 & 1.66 & 10.7130 \\
\hline 520.13 & 1.34 & 27.09 & 20.29 & 12.26 & 3.35 & 10.1970 \\
\hline 525.46 & 1.00 & 25.50 & 25.50 & 12.49 & 2.71 & 9.4190 \\
\hline 456.20 & 1.31 & 26.78 & 20.50 & 11.73 & 3.14 & 6.8400 \\
\hline 468.6339 & 1.7363 & 30.3822 & 17.4982 & 12.0923 & 7.0839 & 99.4850 \\
\hline 459.7555 & 1.3033 & 26.4395 & 20.2870 & 11.4271 & 1.8524 & 46.1390 \\
\hline 671.0602 & 1.0000 & 29.7762 & 29.7762 & 14.1421 & 1.6282 & 11.1570 \\
\hline 504.7391 & 1.1253 & 26.7298 & 23.7537 & 12.2484 & 2.4358 & 10.9750 \\
\hline 514.2094 & 1.0841 & 25.3312 & 23.3670 & 12.3166 & 2.2577 & 12.4230 \\
\hline 446.1420 & 1.0000 & 22.3533 & 22.3533 & 11.5191 & 2.3569 & 11.2200 \\
\hline 426.0178 & 1.0721 & 20.2609 & 18.8975 & 11.1532 & 2.1963 & 35.7050 \\
\hline 423.0583 & 1.6708 & 28.1178 & 16.8293 & 11.4429 & 5.9909 & 98.1560 \\
\hline 737.9437 & 1.9448 & 38.4287 & 19.7593 & 14.8512 & 11.5347 & $7 \quad 18.5320$ \\
\hline 504.1472 & 1.0375 & 25.7893 & 24.8580 & 12.2096 & 2.1089 & 96.9000 \\
\hline 562.1524 & 1.0926 & 25.5004 & 23.3401 & 12.7735 & 1.5068 & 10.4440 \\
\hline 626.0765 & 1.7723 & 37.7268 & 21.2867 & 14.0071 & 11.9240 & 15.5310 \\
\hline 444.9583 & 1.0000 & 23.0347 & 23.0347 & 11.4245 & 1.5698 & 88.4590 \\
\hline 427.7935 & 1.0000 & 21.2867 & 21.2867 & 11.1655 & 1.6755 & 12.0010 \\
\hline 415.3638 & 2.0687 & 31.0859 & 15.0267 & 11.3523 & 9.2711 & 17.7910 \\
\hline
\end{tabular}




\begin{tabular}{ccccccc}
534.9255 & 1.0310 & 25.2444 & 24.4858 & 12.7001 & 2.8860 & 10.9510 \\
691.1844 & 1.3684 & 35.5063 & 25.9477 & 14.6260 & 5.8700 & 16.2770 \\
514.2094 & 1.4102 & 27.5567 & 19.5409 & 12.1605 & 4.1793 & 6.3610 \\
444.9583 & 1.0738 & 22.7511 & 21.1884 & 11.5378 & 2.8463 & 8.5550 \\
475.1446 & 1.3893 & 27.6462 & 19.9000 & 11.9212 & 4.3961 & 10.9650 \\
494.6770 & 1.0000 & 25.0686 & 25.0686 & 12.0026 & 0.9314 & 8.9800 \\
479.2879 & 1.1763 & 24.2584 & 20.6226 & 11.8091 & 2.4841 & 9.7150 \\
584.0524 & 1.1339 & 26.9886 & 23.8015 & 13.0513 & 2.6182 & 12.9830 \\
678.1628 & 1.1289 & 30.1384 & 26.6969 & 14.0391 & 1.7422 & 13.1530 \\
856.3217 & 1.5543 & 37.0638 & 23.8465 & 15.8469 & 8.2670 & 10.8610 \\
582.8686 & 1.5518 & 31.5810 & 20.3517 & 13.2787 & 6.2631 & 4.8260 \\
630.2198 & 1.6099 & 32.8632 & 20.4135 & 13.5947 & 7.1258 & 14.4650 \\
443.7745 & 1.0000 & 23.9929 & 23.9929 & 11.6009 & 3.3615 & 7.7630 \\
552.0903 & 1.0000 & 25.3410 & 25.3410 & 12.8485 & 3.1034 & 10.9800 \\
496.4527 & 1.0000 & 25.1713 & 25.1713 & 12.1781 & 2.3412 & 10.8150 \\
501.1878 & 1.0000 & 24.0883 & 24.0883 & 12.1034 & 1.3417 & 13.1830 \\
454.4285 & 1.0000 & 25.5594 & 25.5594 & 11.5097 & 1.0172 & 7.5730 \\
671.6520 & 1.3153 & 30.6987 & 23.3401 & 14.2797 & 6.9476 & 14.9100 \\
662.7737 & 2.4538 & 40.8097 & 16.6315 & 14.8398 & 17.7264 & 14.3110 \\
659.2224 & 1.2428 & 31.3146 & 25.1967 & 14.0829 & 5.5969 & 13.7600 \\
337.2343 & 1.5692 & 22.4792 & 14.3249 & 10.0383 & 5.1795 & 13.5030 \\
692.9601 & 1.3256 & 35.9730 & 27.1377 & 14.4843 & 4.6877 & 13.3720 \\
464.4906 & 1.8380 & 28.4393 & 15.4732 & 12.1970 & 7.8637 & 7.4730 \\
388.1368 & 1.4175 & 27.9771 & 19.7370 & 10.8048 & 3.3067 & 11.0270 \\
640.2819 & 1.7518 & 36.0891 & 20.6014 & 14.3510 & 12.2876 & 11.2180 \\
397.0152 & 1.5948 & 26.5235 & 16.6315 & 10.9940 & 4.9561 & 5.4000 \\
537.8850 & 1.0211 & 24.3025 & 23.8015 & 12.5737 & 3.4042 & 10.4600 \\
550.3146 & 2.7791 & 38.4114 & 13.8214 & 14.0220 & 22.9717 & 6.9080 \\
661.5899 & 1.1436 & 28.2243 & 24.6804 & 13.8712 & 2.6678 & 10.2410 \\
597.0739 & 1.4657 & 30.8348 & 21.0370 & 13.4677 & 4.0772 & 10.4360 \\
415.9557 & 2.0901 & 28.3562 & 13.5668 & 11.3975 & 7.2165 & 4.7590 \\
544.3957 & 1.0804 & 27.3999 & 25.3614 & 12.7614 & 2.9944 & 12.0180 \\
453.8366 & 1.9514 & 31.3006 & 16.0404 & 12.1155 & 9.9215 & 11.0930 \\
513.0256 & 1.1578 & 24.8785 & 21.4874 & 12.1806 & 1.5118 & 11.2600 \\
329.5398 & 1.4352 & 24.9653 & 17.3953 & 10.0674 & 4.4330 & 5.6810 \\
& & & & & & \\
\hline 50
\end{tabular}




\section{APPENDIX II}

\section{Histogram of Model Testing Samples}

(Total of 8 batches)

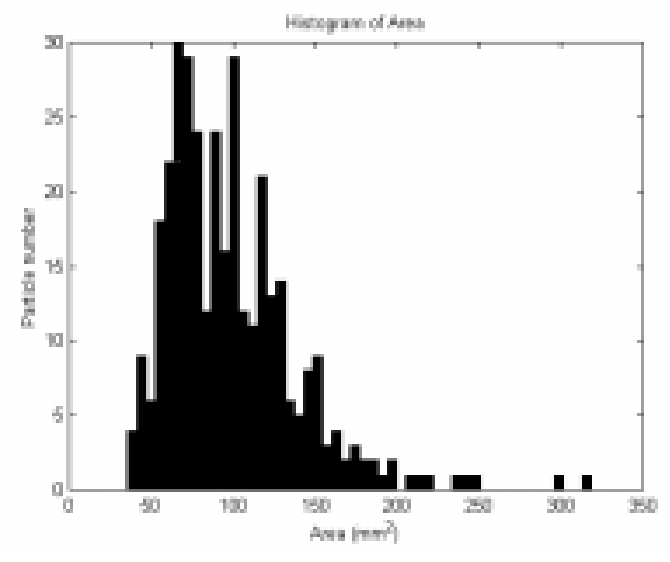

(a) projected area $\left(\mathrm{mm}^{2}\right)$

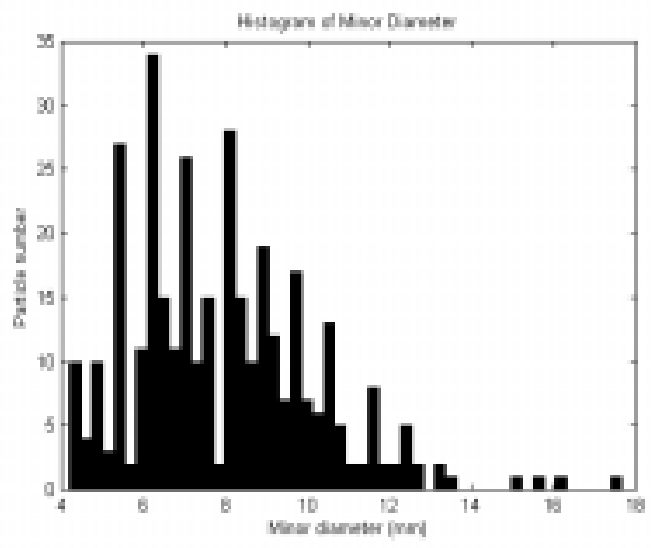

(c) minor diameter $(m)$

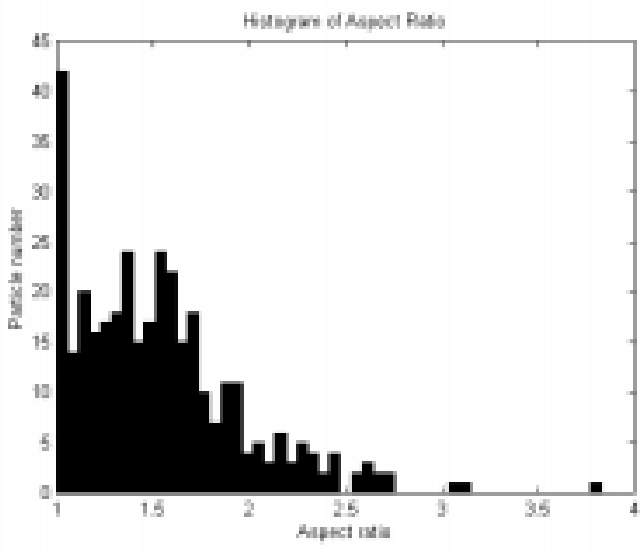

(b) aspect ratio

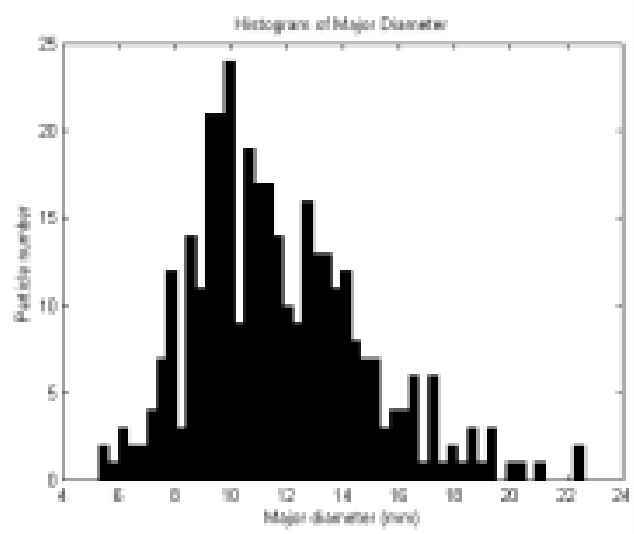

(d) major diameter $(m)$

APPENDIX II-1 For Sample Batch \#1, Image $a, 345$ particles 


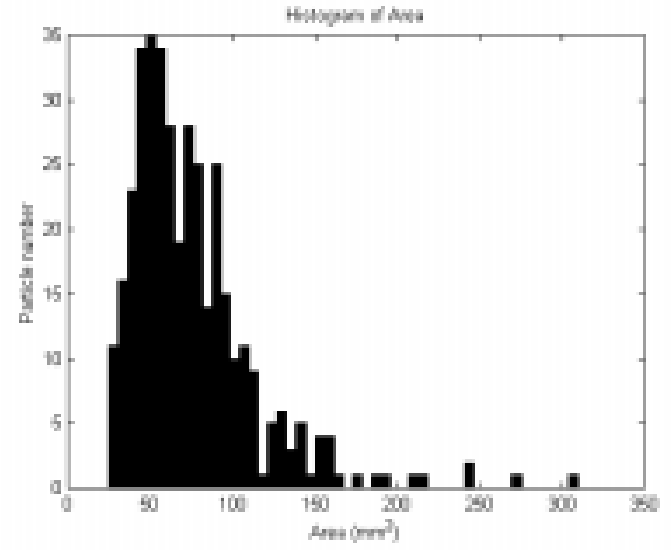

(a) projected area $\left(\mathrm{mm}^{2}\right)$

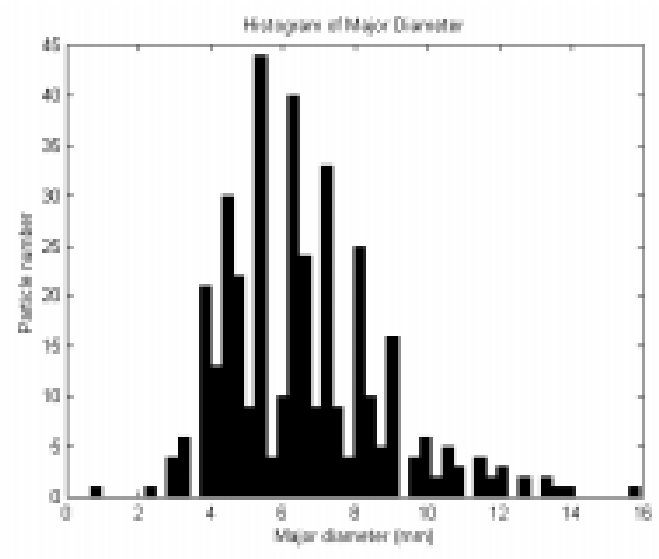

(c) minor diameter $(m)$

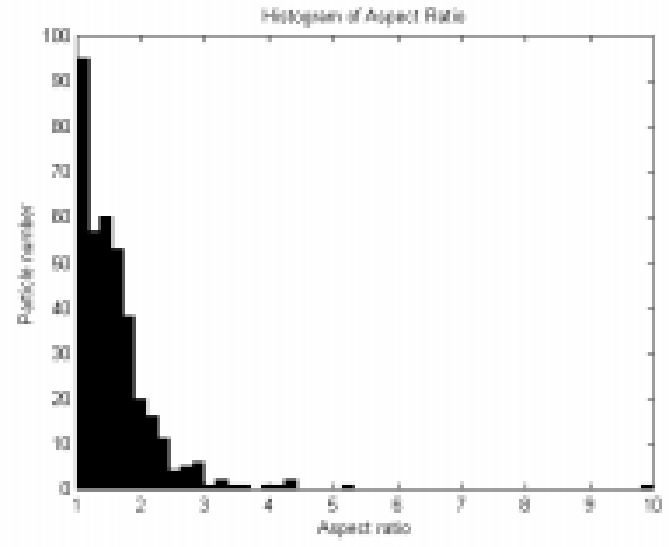

(b) aspect ratio

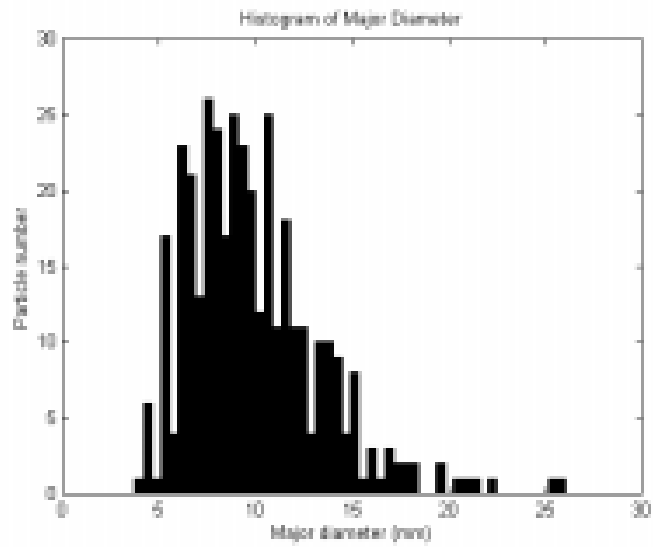

(d) major diameter $(m)$

APPENDIX II-2 For Sample Batch \#2, Image $a$, 376 particles 


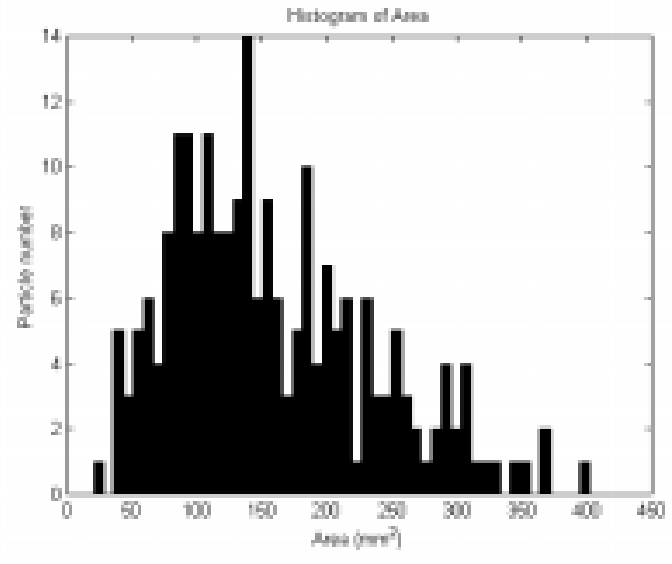

(a) projected area $\left(\mathrm{mm}^{2}\right)$

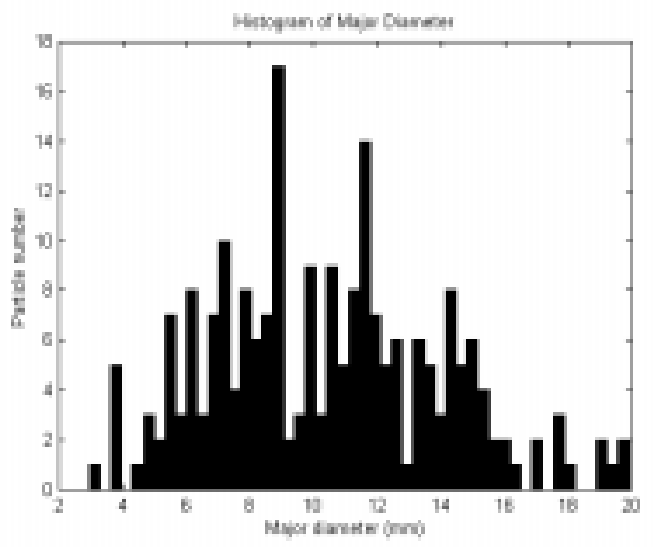

(c) minor diameter $(m)$

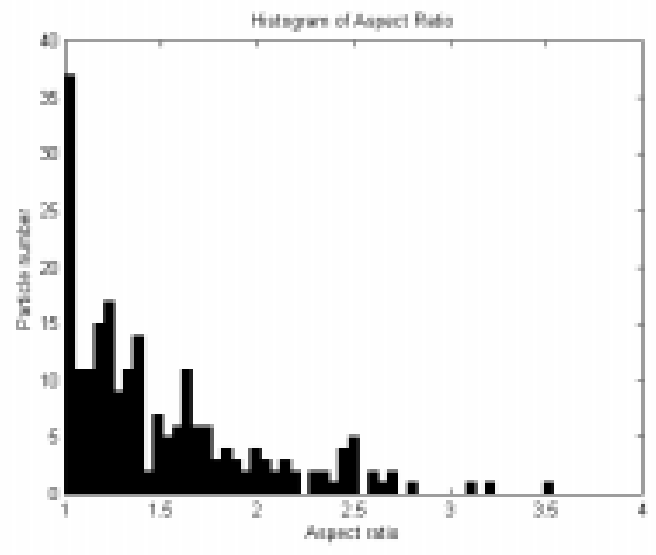

(b) aspect ratio

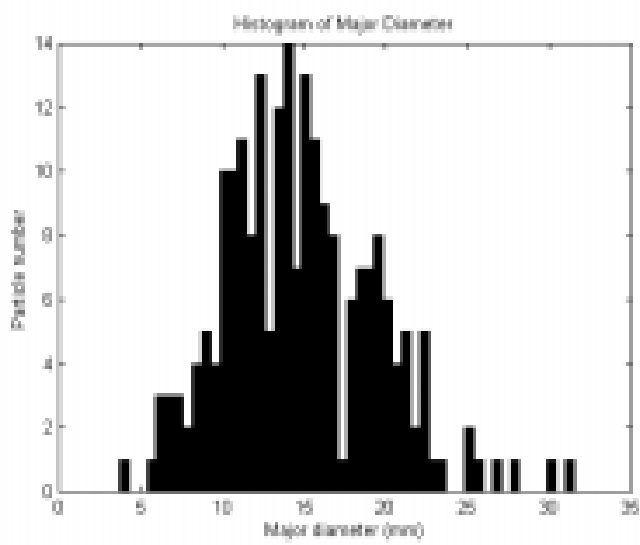

(d) major diameter $(m)$

APPENDIX II-3 For Sample Batch \#3, Image $a, 215$ particles 


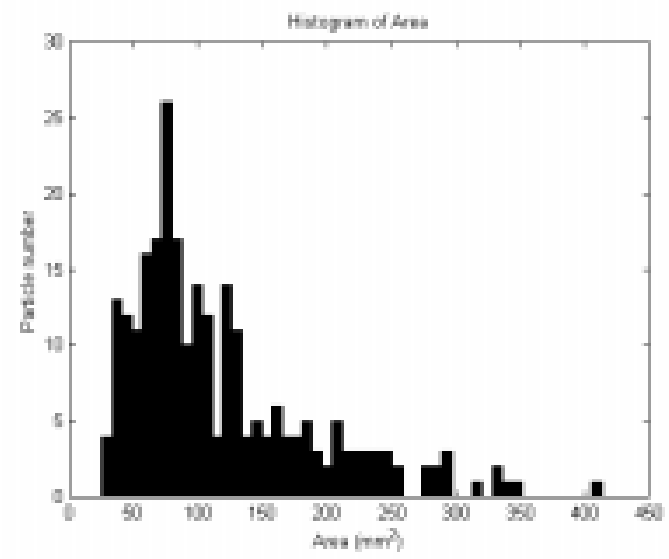

(a) projected area $\left(\mathrm{mm}^{2}\right)$

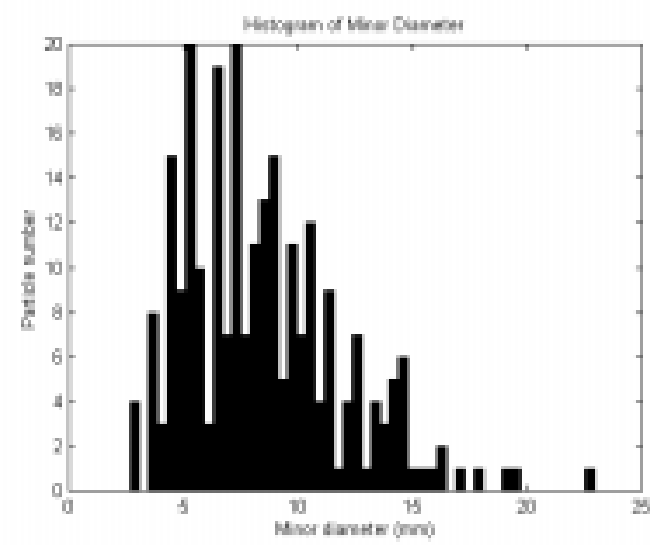

(c) minor diameter $(\mathrm{m})$

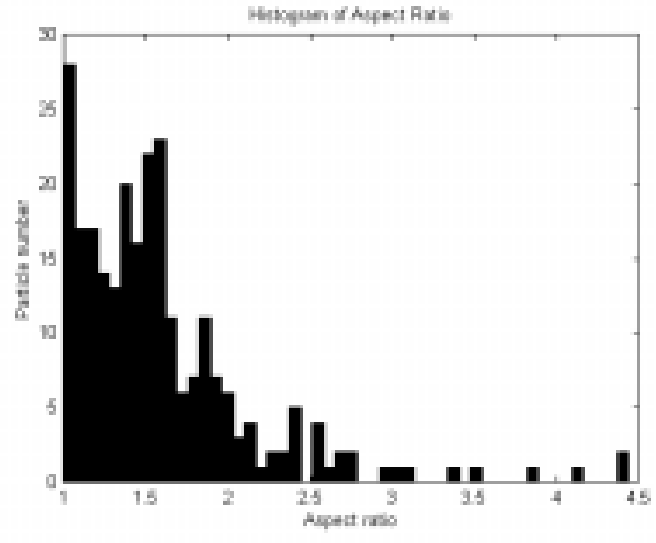

(b) aspect ratio

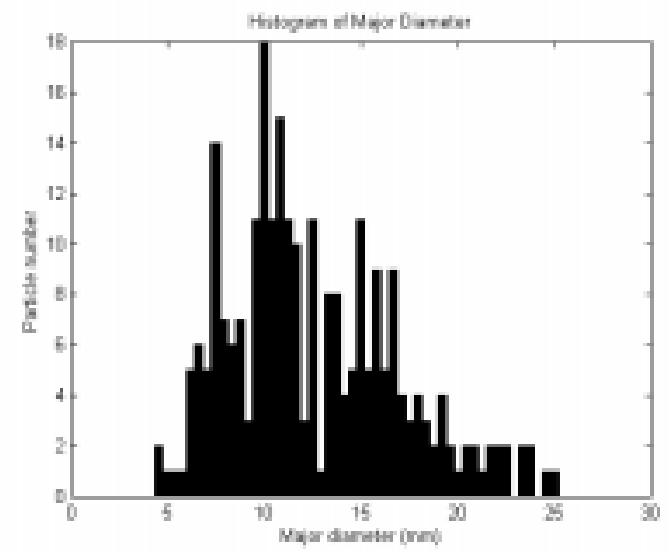

(d) major diameter $(\mathrm{m})$

APPENDIX II-4 For Sample Batch \#4, Image $a$, 251 particles 


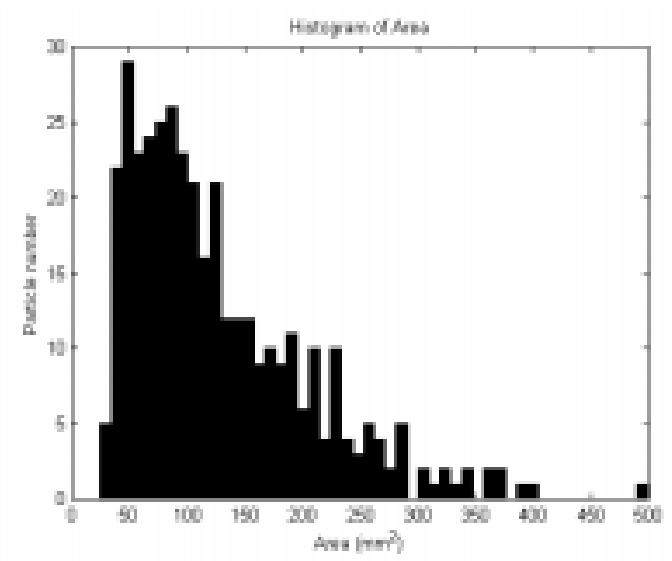

(a) projected area $\left(\mathrm{mm}^{2}\right)$

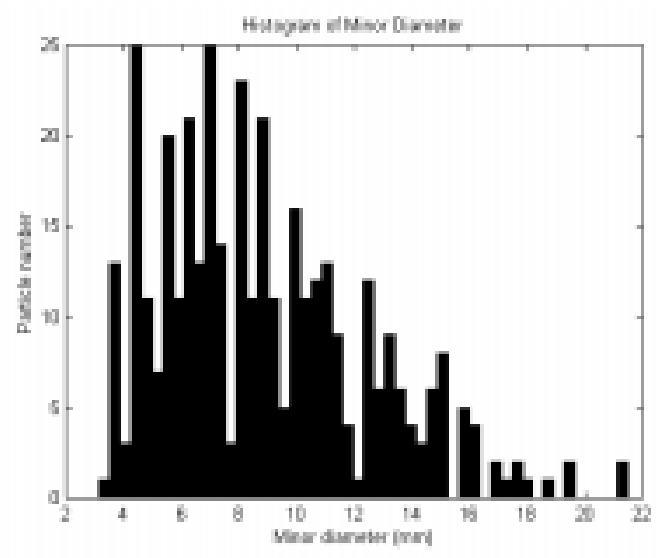

(c) minor diameter $(m)$

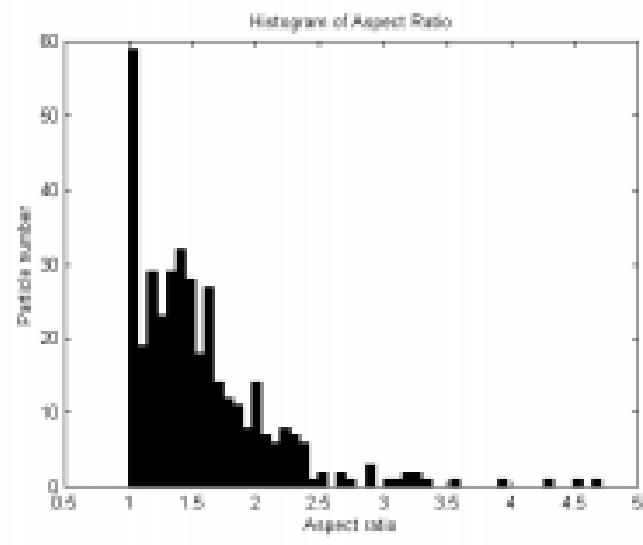

(b) aspect ratio

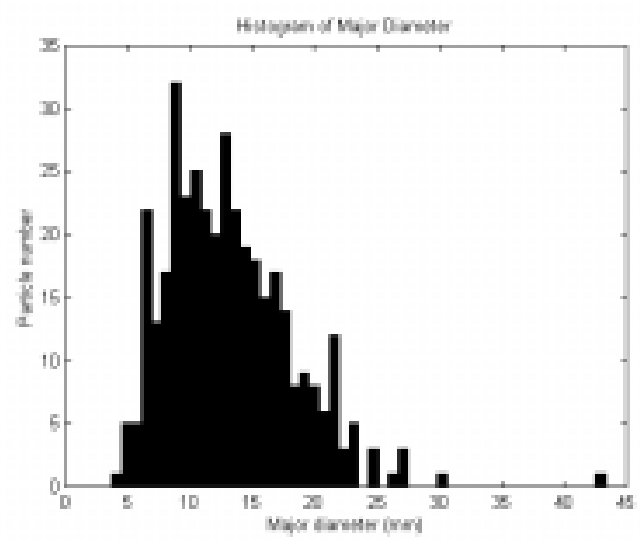

(d) major diameter $(m)$

APPENDIX II-5 For Sample Batch \#5, Image $a$, 378 particles 


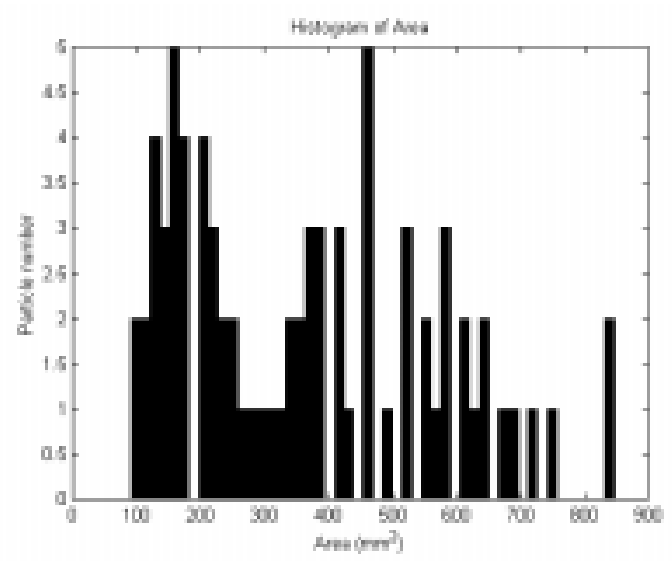

(a) projected area $\left(\mathrm{mm}^{2}\right)$

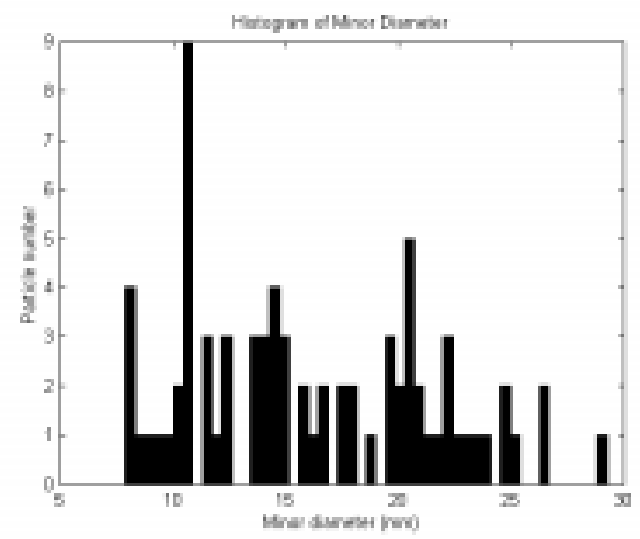

(c) minor diameter $(m)$

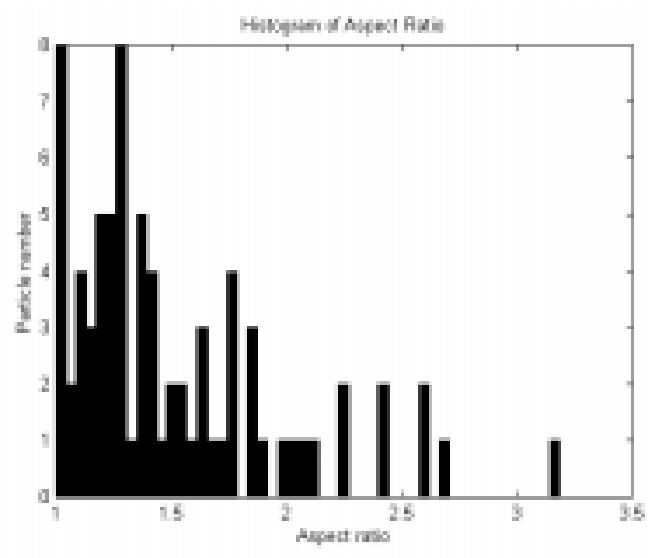

(b) aspect ratio

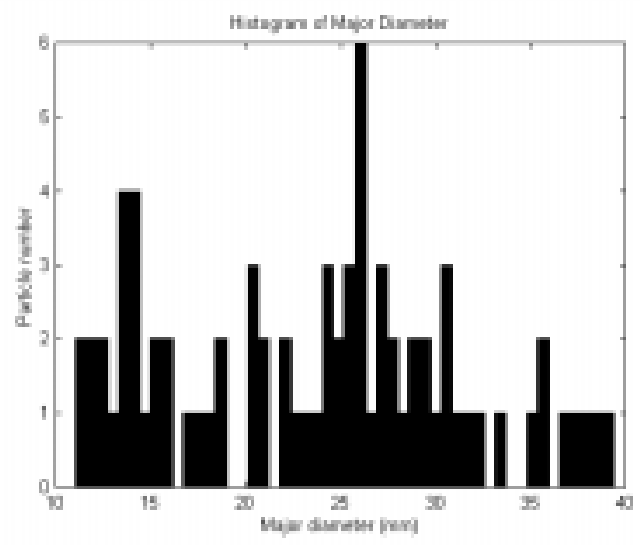

(d) major diameter $(m)$

APPENDIX II-6 For Sample Batch \#6, Image $a$, 76 particles 


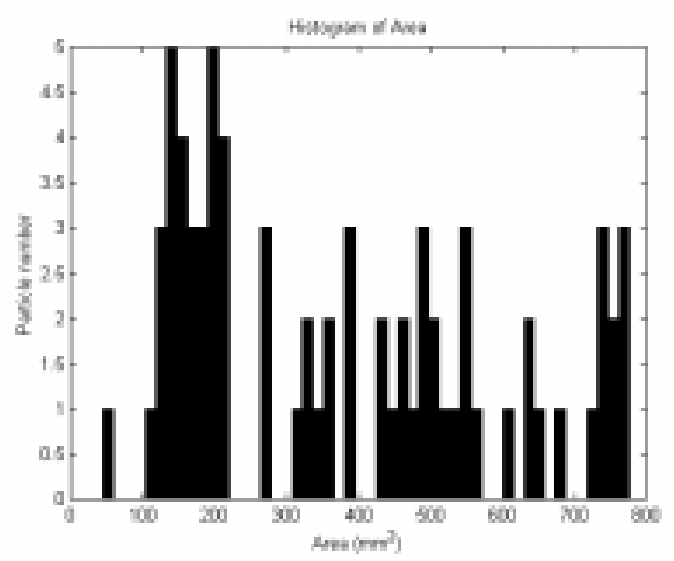

(a) projected area $\left(\mathrm{mm}^{2}\right)$

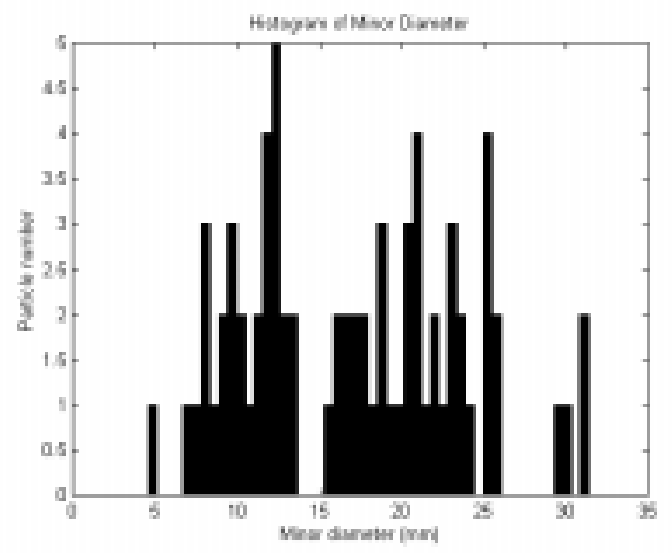

(c) minor diameter $(\mathrm{m})$

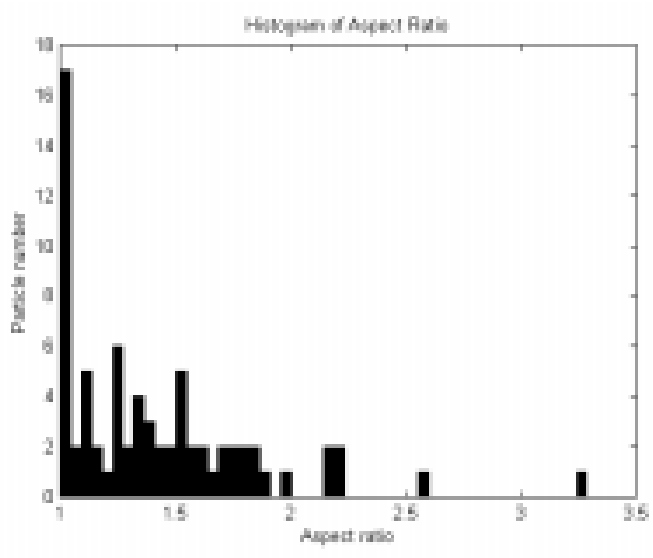

(b) aspect ratio

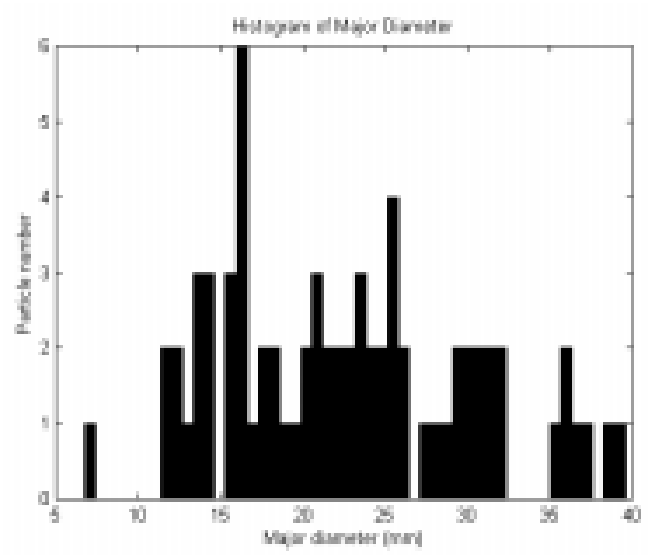

(d) major diameter $(m)$

APPENDIX II-7 For Sample Batch \#7, Image $a$, 71 particles 


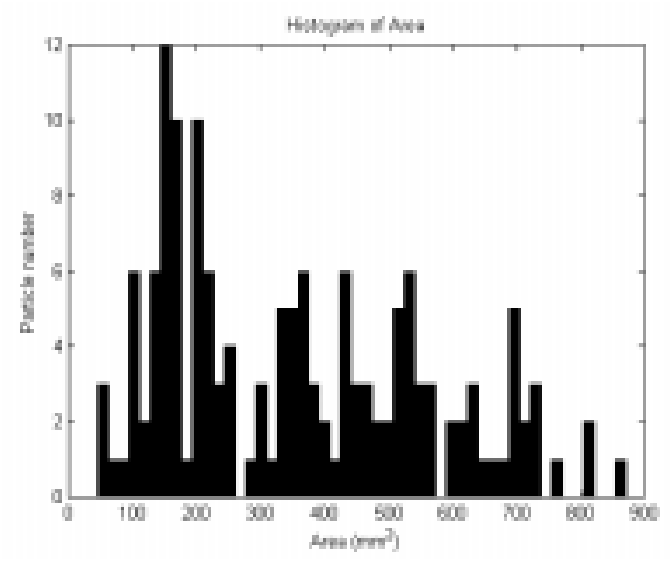

(a) projected area $\left(\mathrm{mm}^{2}\right)$

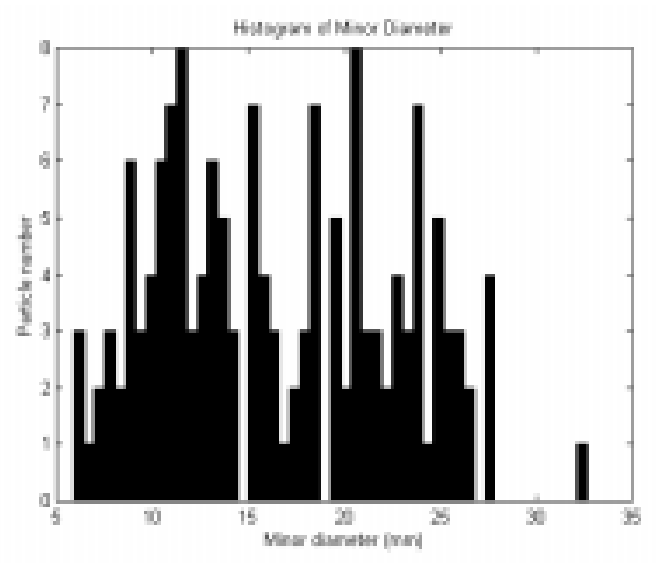

(c) minor diameter $(m)$

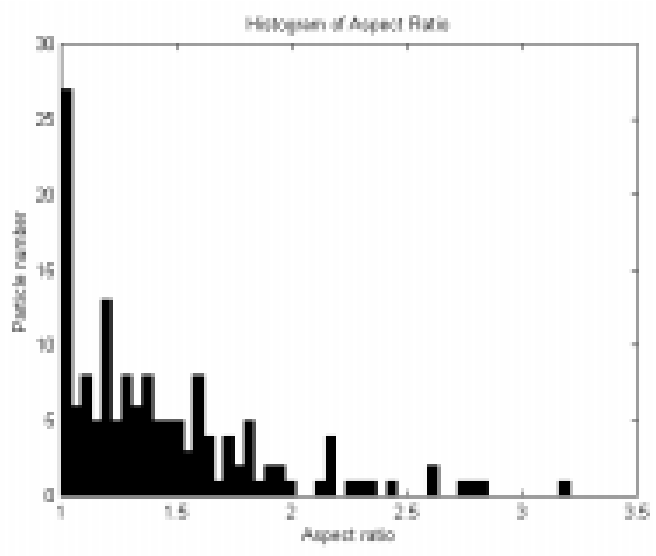

(b) aspect ratio

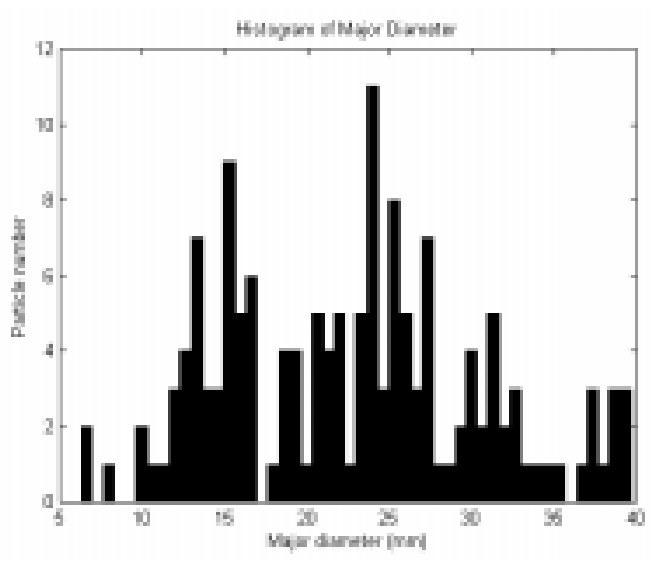

(d) major diameter $(m)$

APPENDIX II-8 For Sample Batch \#8, Image $a$, 150 particles 


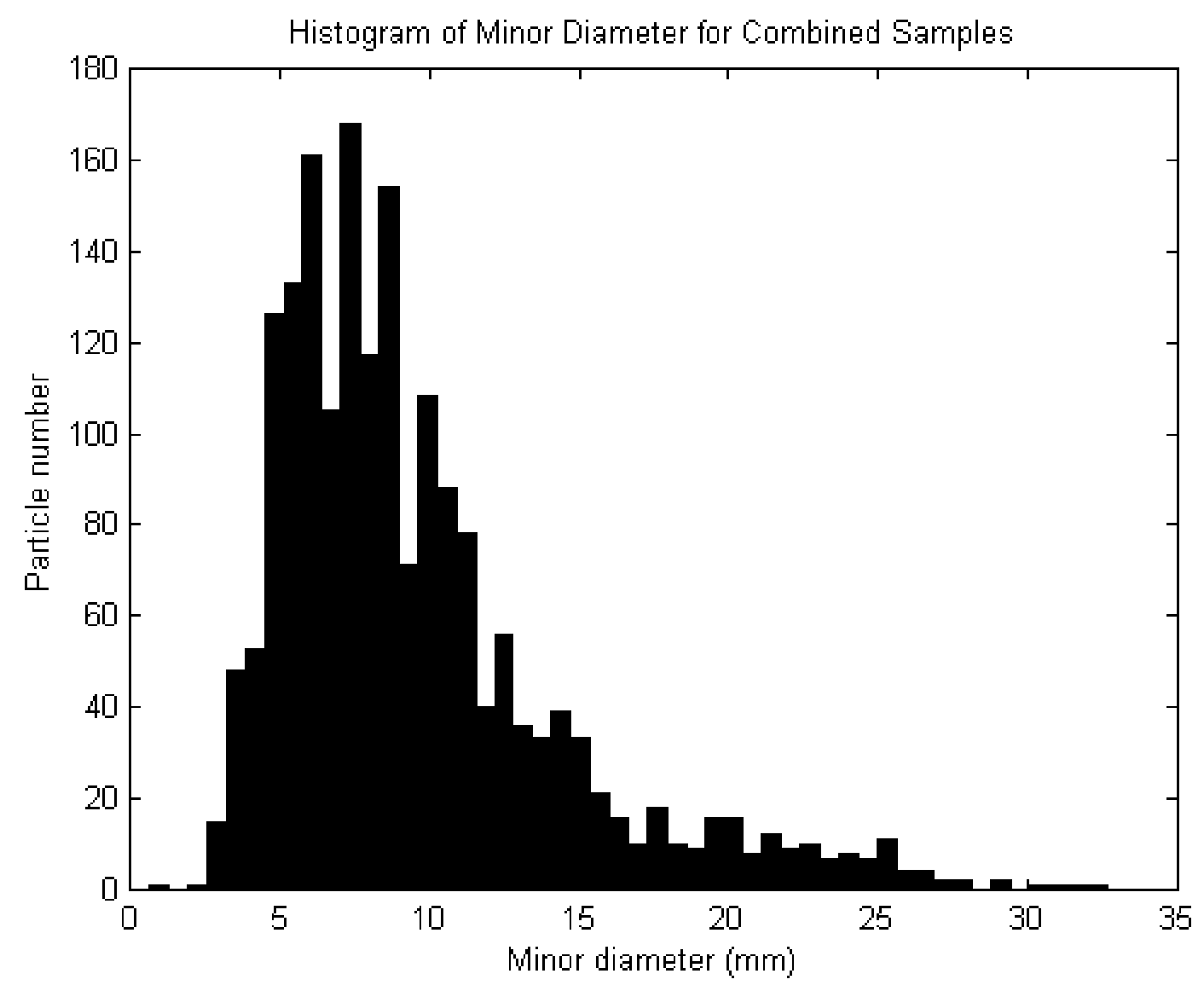

APPENDIX II-9 Histogram of Minor Diameter For Combined Sample Batches, Image $a, 1862$ particles 


\section{APPENDIX III}

\section{Benchmark of Samples}

(Total of 8 batches)

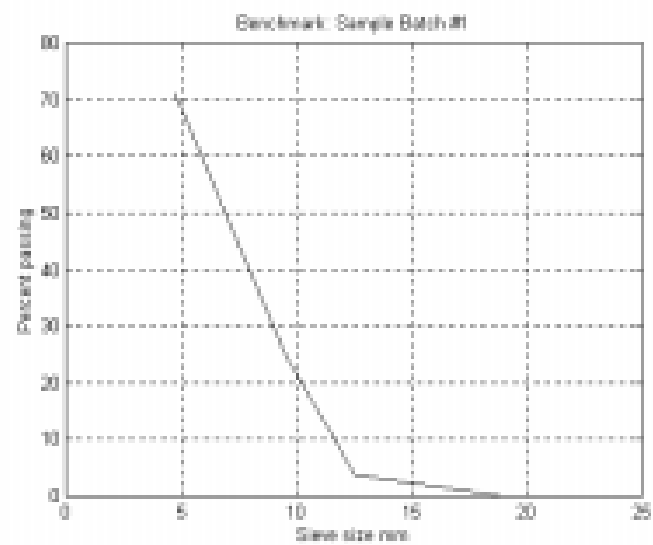

(a) percent retained

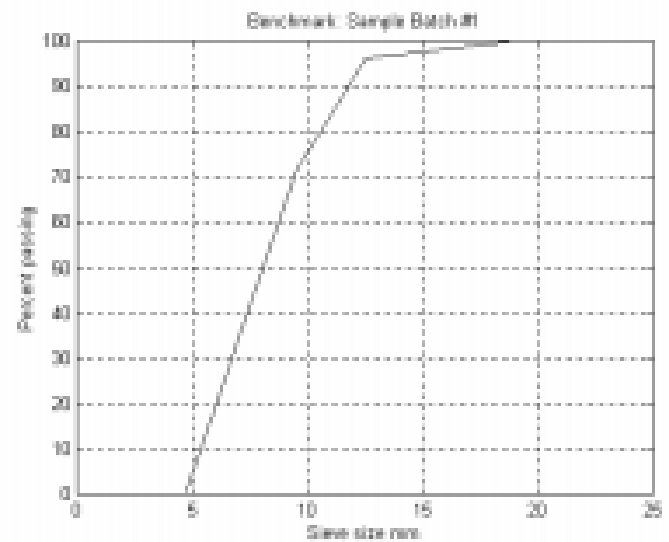

(b) percent passing

APENDIX III-1 Benchmark for Sample Batch \#1

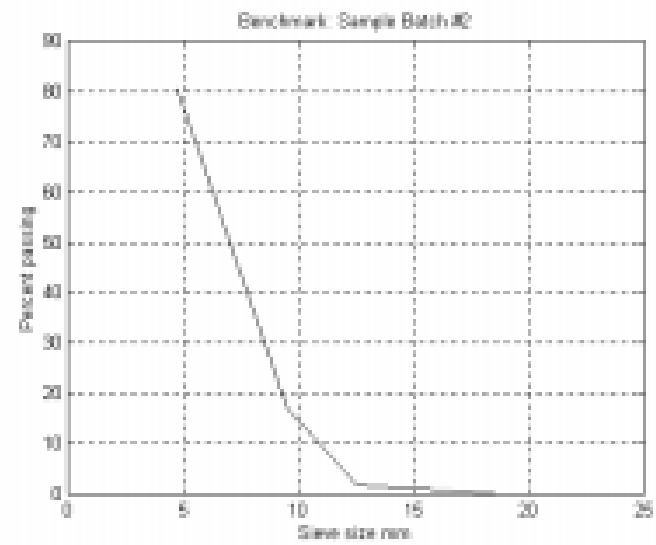

(a) percent retained

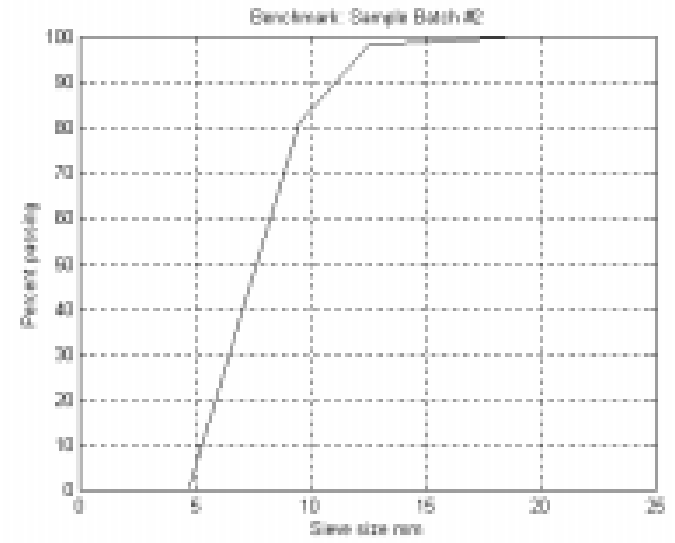

(b) percent passing

APENDIX III-2 Benchmark for Sample Batch \#2 


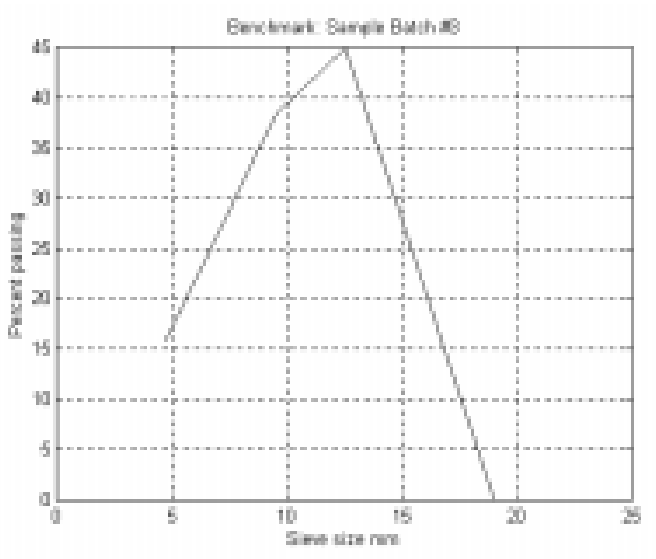

(a) percent retained

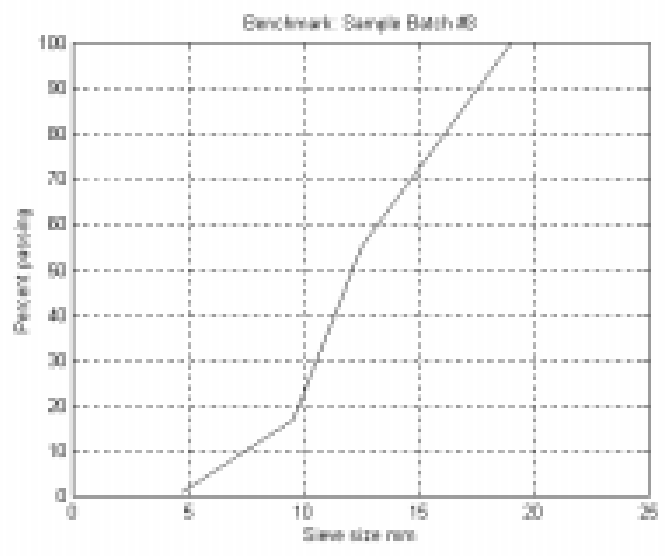

(b) percent passing

\section{APENDIX III-3 Benchmark for Sample Batch \#3}

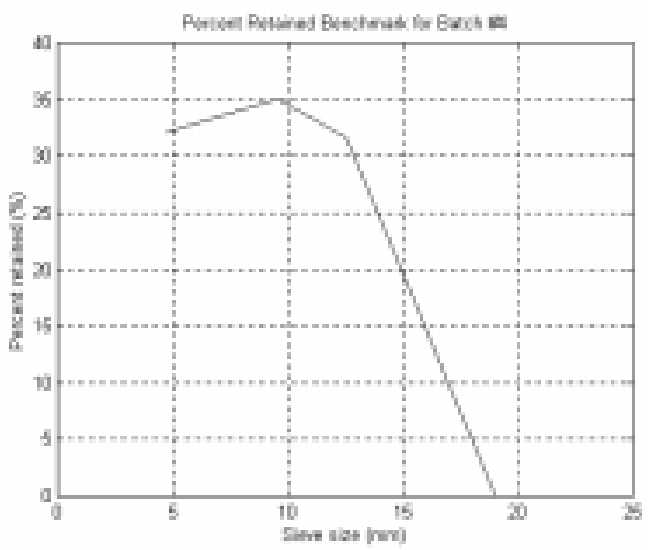

(a) percent retained

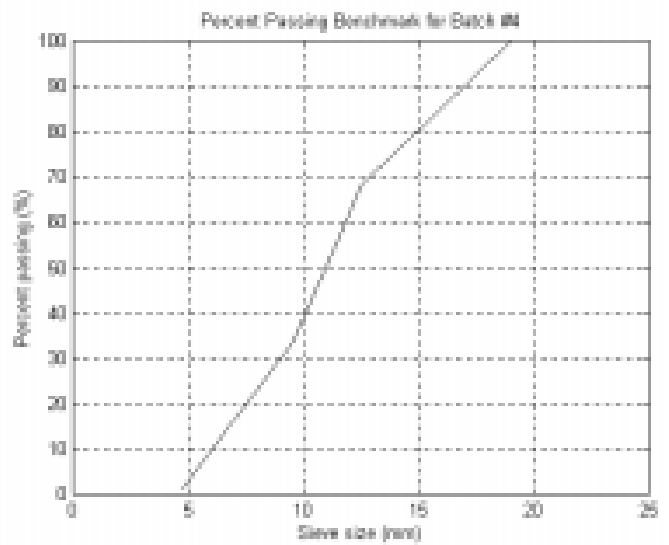

(b) percent passing

\section{APENDIX III-4 Benchmark for Sample Batch \#4}




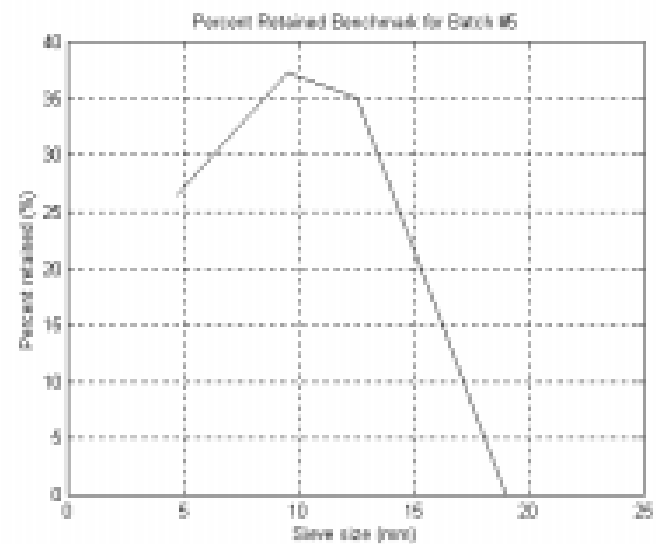

(a) percent retained

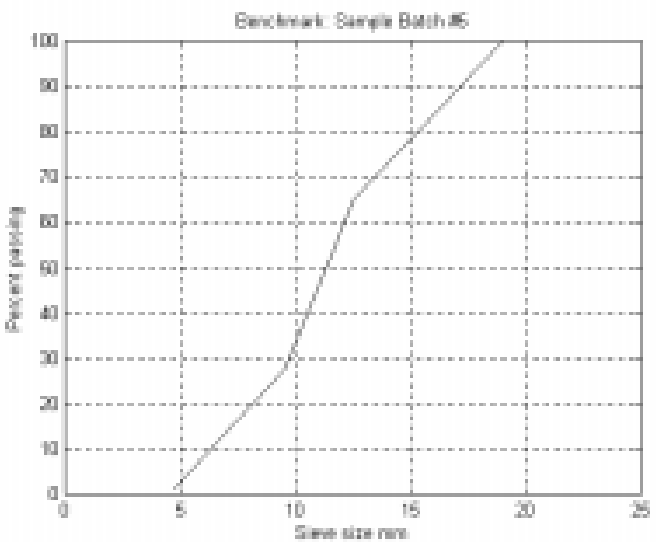

(b) percent passing

\section{APENDIX III-5 Benchmark for Sample Batch \#5}

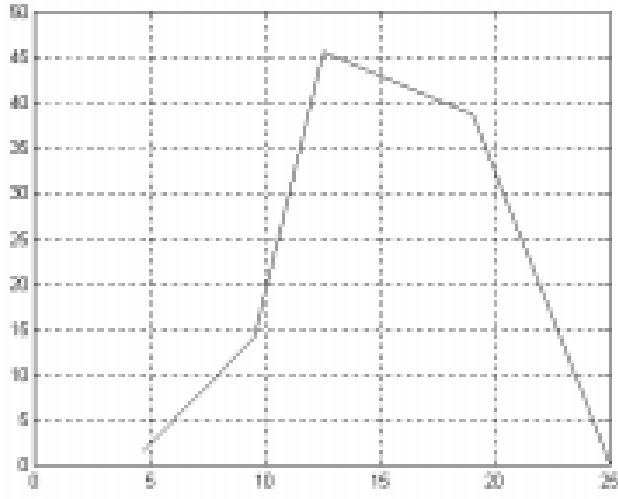

(a) percent retained

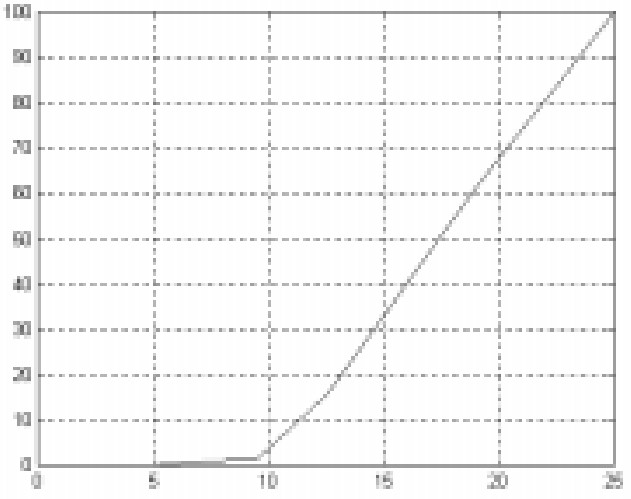

(b) percent passing

APENDIX III-6 Benchmark for Sample Batch \#6 


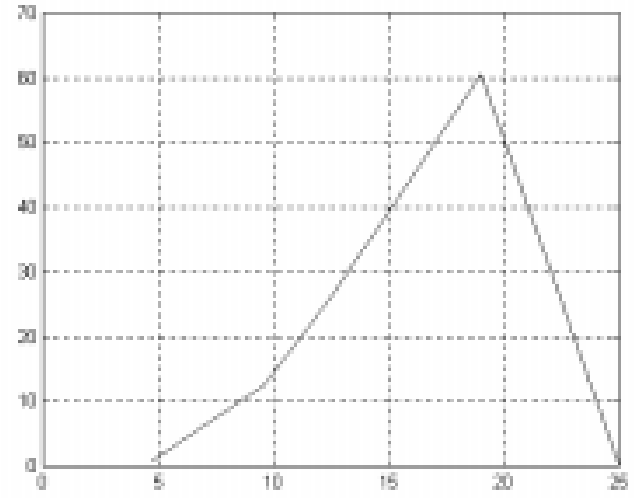

(a) percent retained

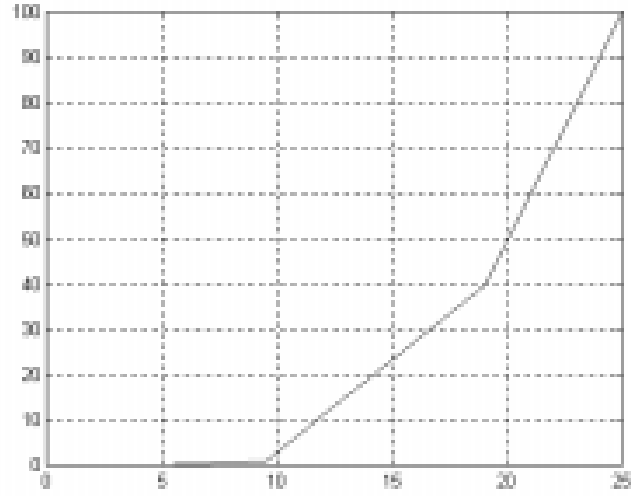

(b) percent passing

\section{APENDIX III-7 Benchmark for Sample Batch \#7}

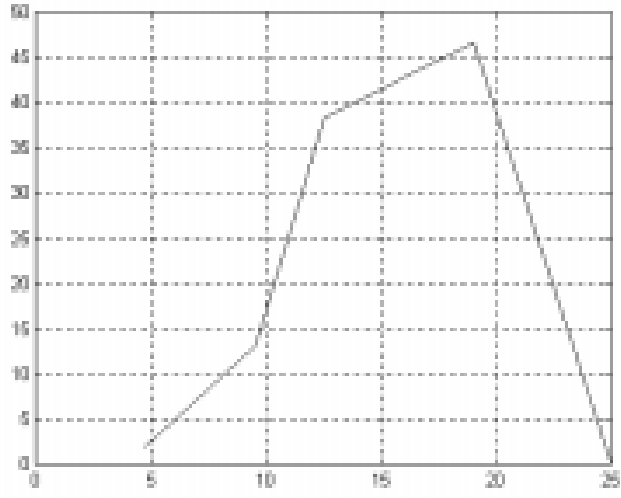

(a) percent retained

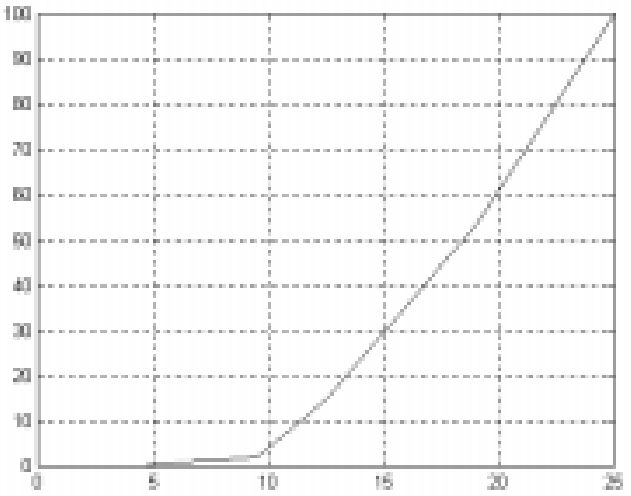

(b) percent passing

APENDIX III-8 Benchmark for Sample Batch \#8 


\section{APPENDIX IV}

Samples for Testing System Performance

(Total of 10 Groups)

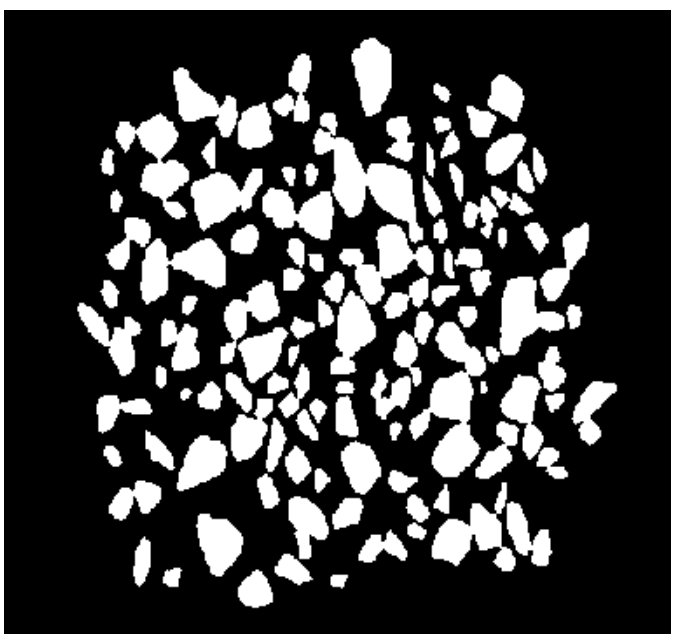

(a-1) group \#1

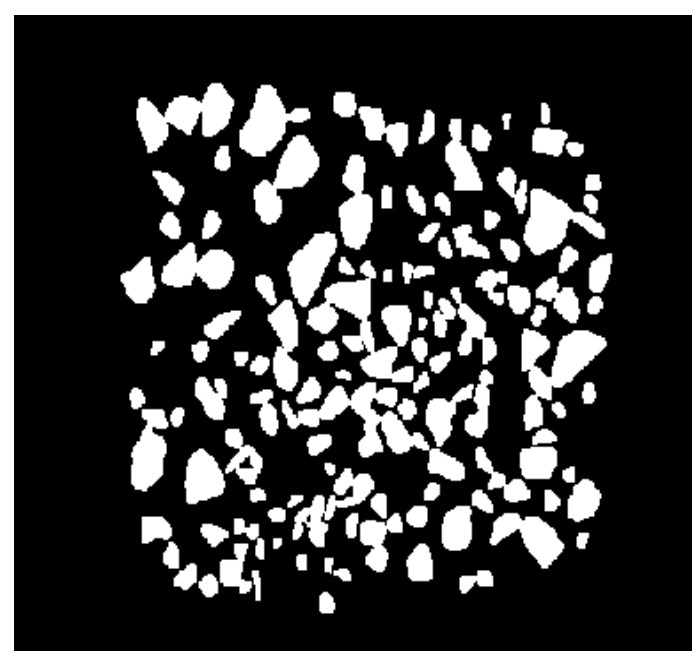

(b-1) group \#2

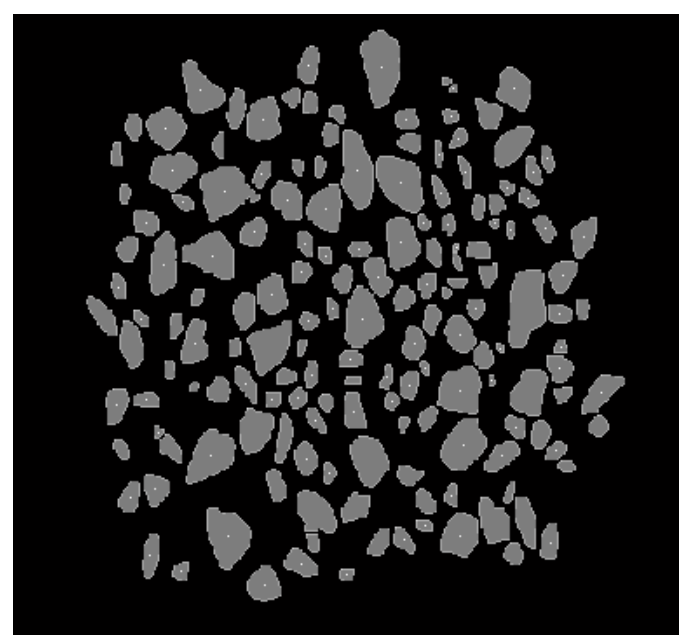

(a-2) processed with $N_{l}=162$

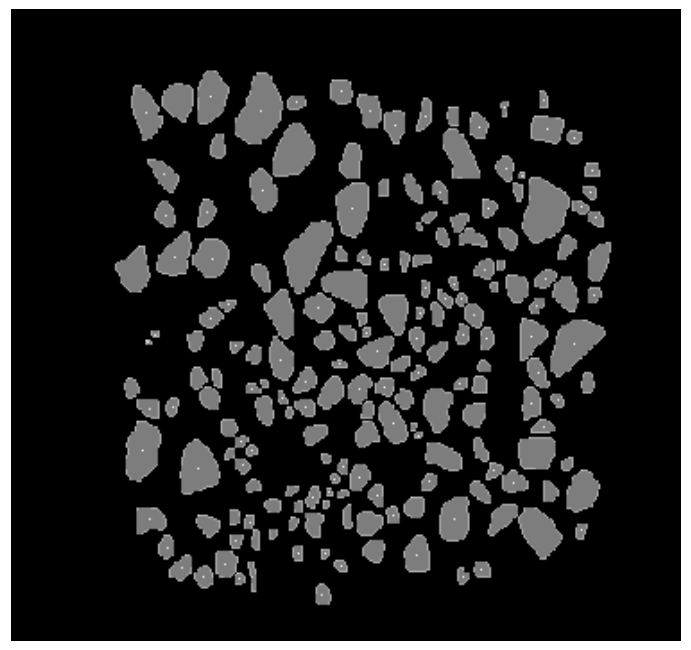

(b-2) processed with $N_{2}=188$ 


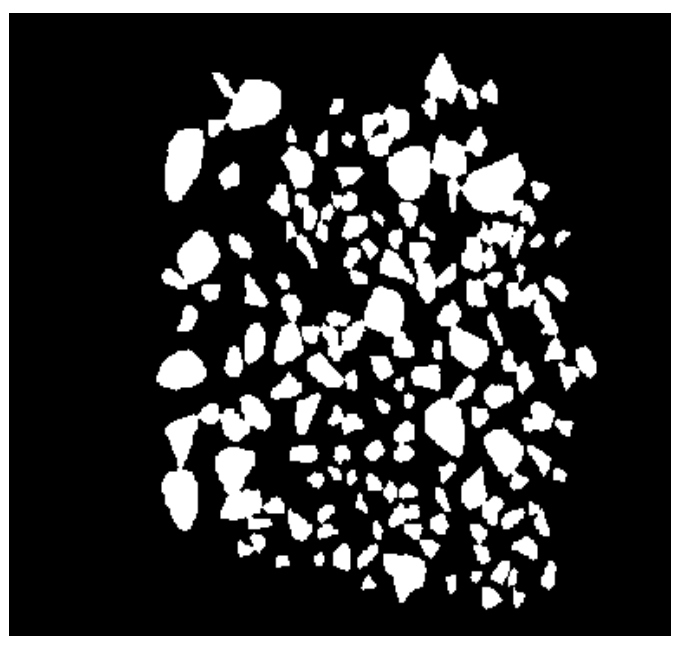

(c-1) group \#3

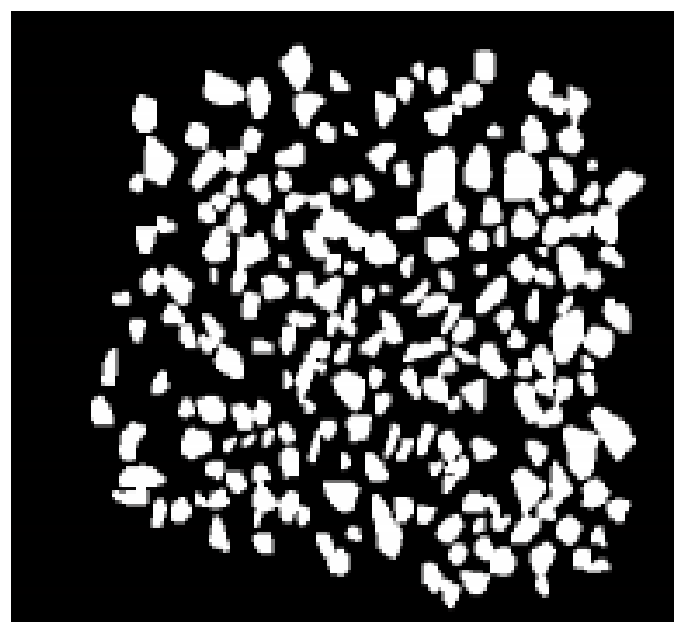

(d-1) group \#4

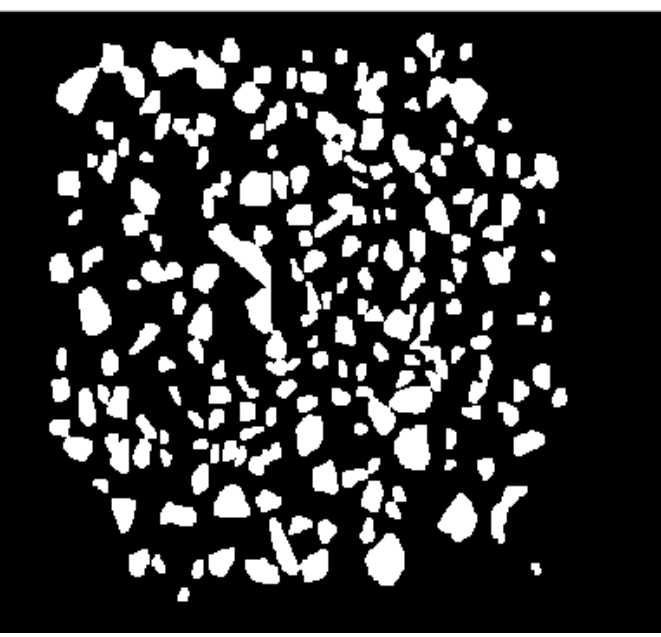

(e-1) group \#1

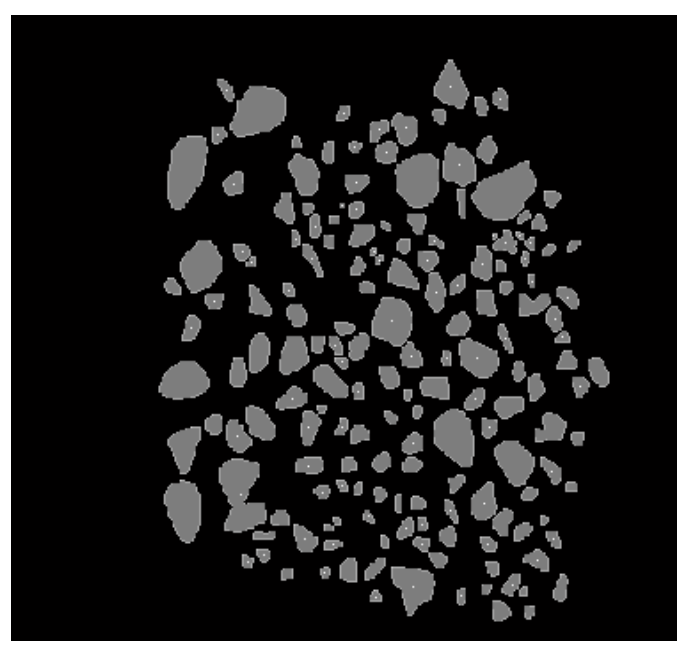

(c-2) processed with $N_{3}=170$

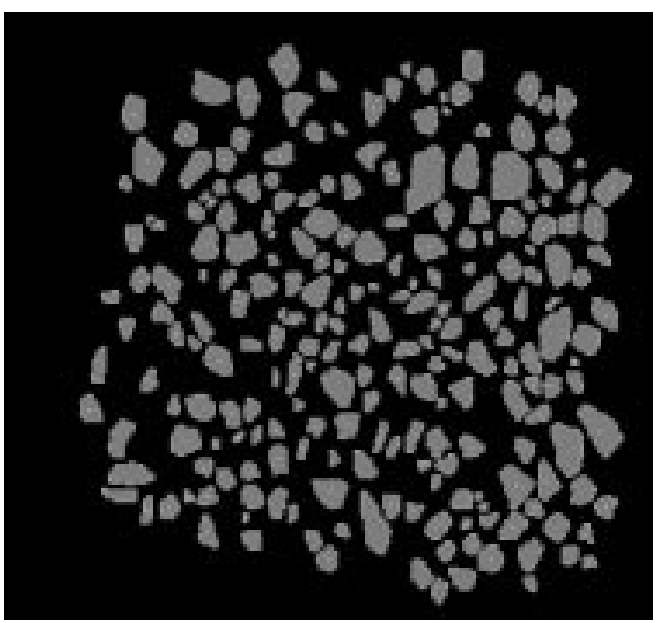

(d-2) processed with $N_{4}=227$

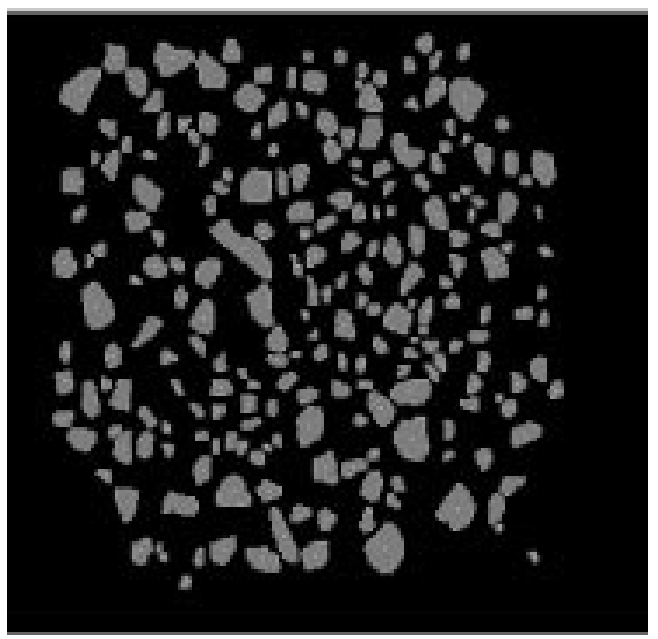

(e-2) processed with $N_{5}=227$ 


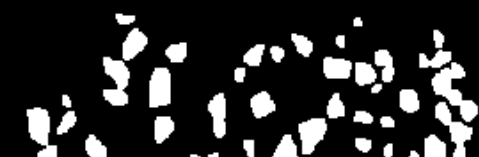

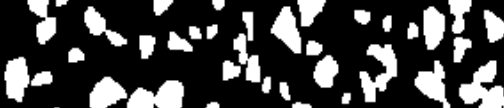

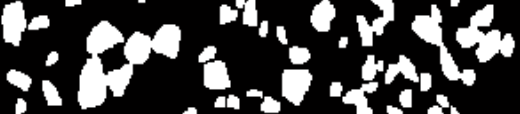

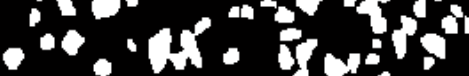

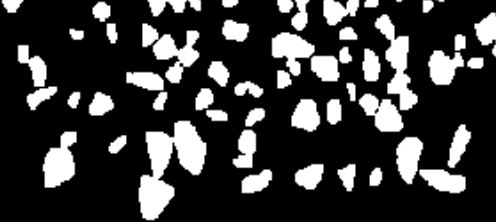

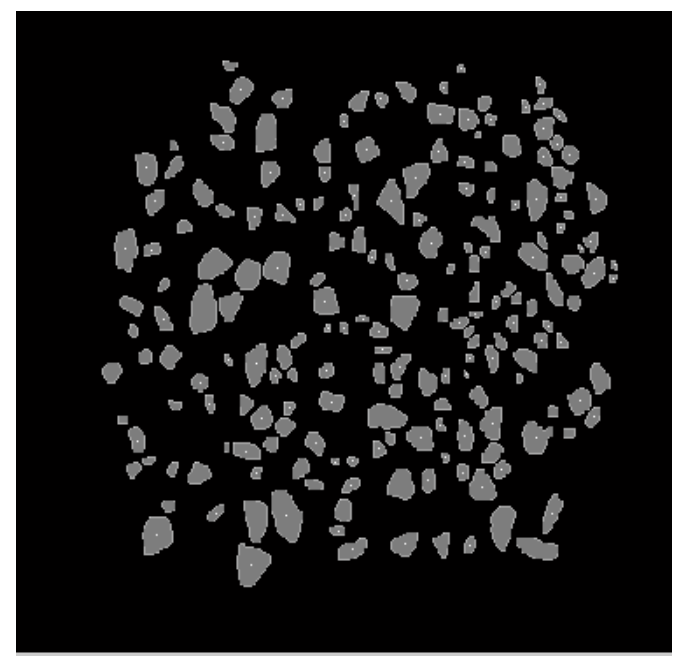

(f-2) processed with $N_{6}=194$

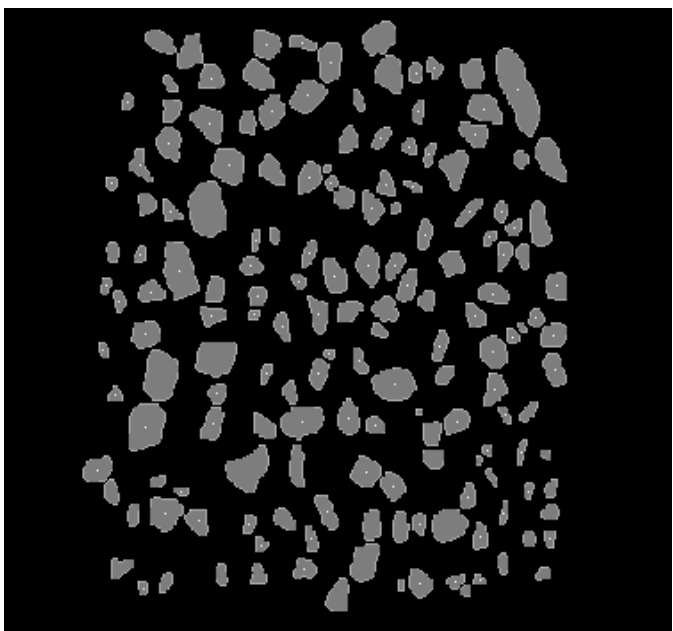

(g-2) processed with $N_{7}=166$

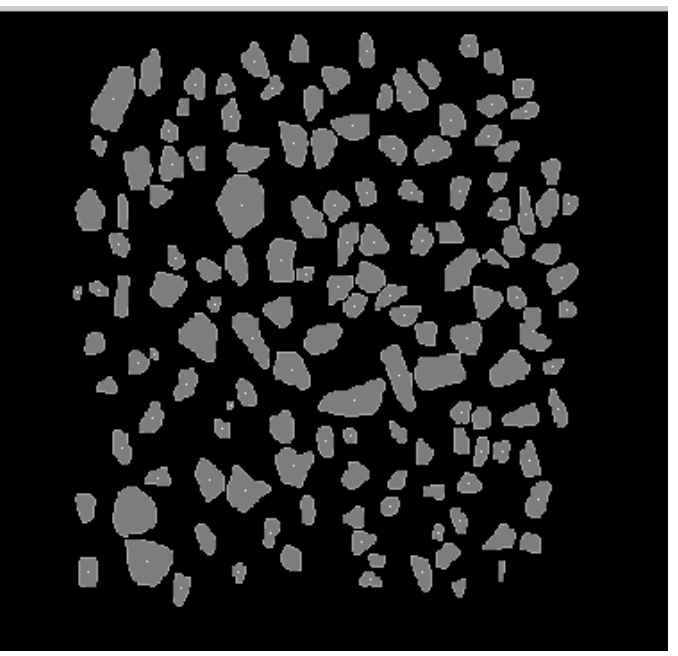

(h-1) group \#8

(h-2) processed with $N_{8}=145$ 


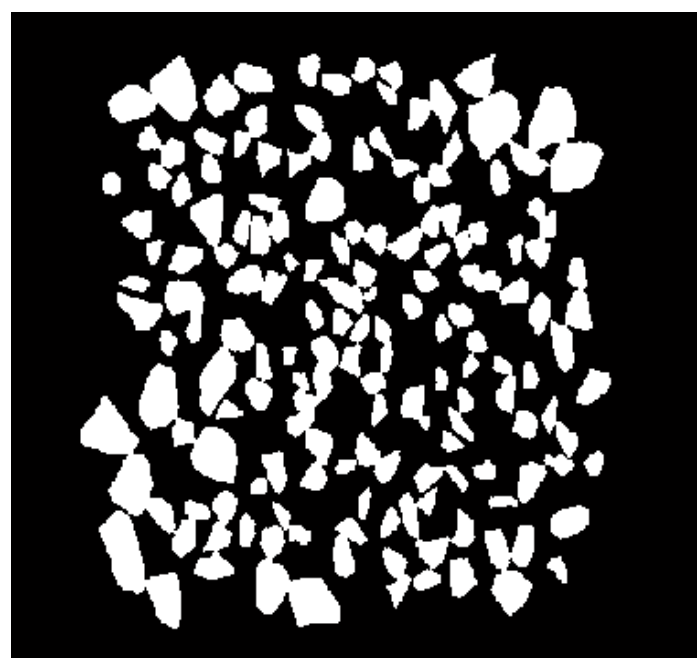

(i-1) group \#9

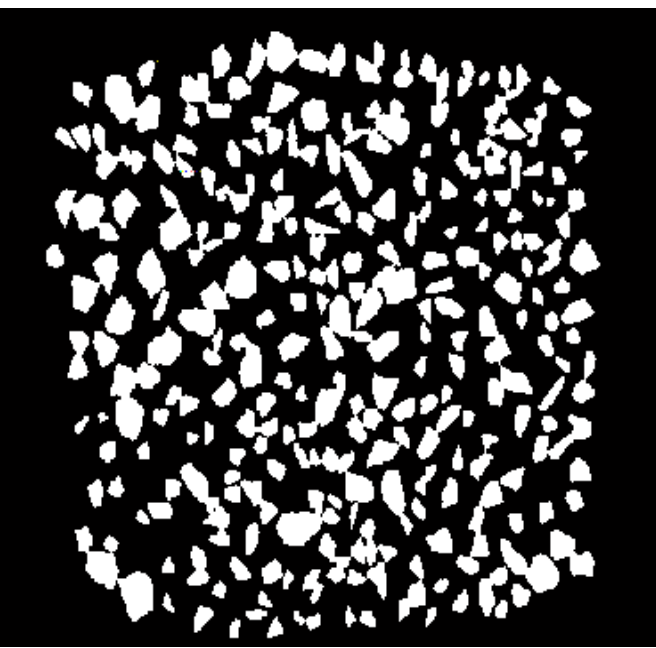

(j-1) group \#10

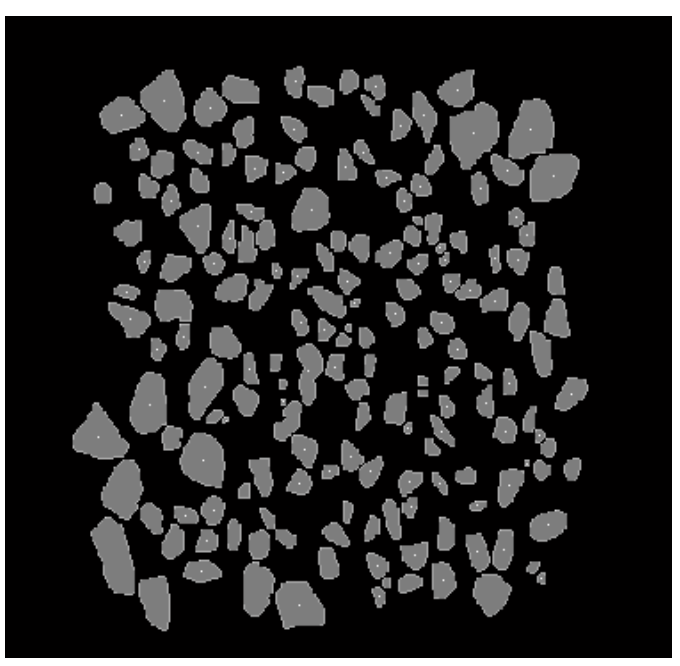

(i-2) processed with $N_{9}=174$

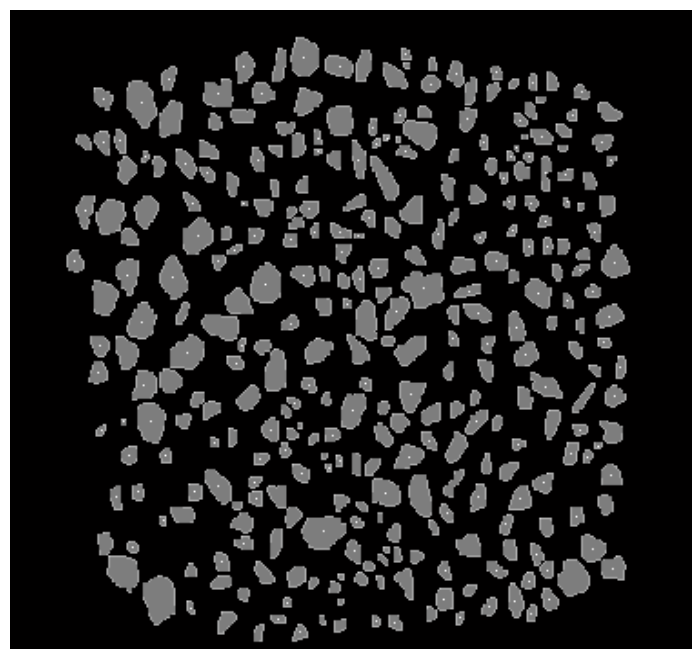

(j-2) processed with $N_{10}=321$ 


\section{VITA}

Ken Cheng was born September 12, 1962 in Wuhan, Hubei, P.R. China. He entered Xiangtan University in 1981, where he studied Mechanical Engineering, graduating with a BSME in 1985. He then worked as a production engineer in Chengdu Seamless Steel Tube Manufacturing Company, Sichuan, until March 1992 when he got a chance to travel to Canada.

In western Canada, Ken lived on a farm and attended Canadian Lutheran College where he improved his English skills and earned a Bachelor of Arts degree. In 1994, Ken enrolled in a MSME program at the University of Akron, Ohio and graduated in 1996. From there, he came immediately to West Virginia University to pursue a Ph.D degree in engineering. Upon graduation from WVU, Ken plans to pursue a research or teaching position in automatic controls or machine vision. 